\title{
Von Amtsgärten und Vogelkojen
}

\section{Beiträge zum Göttinger Umwelthistorischen Kolloquium $2011-2012$}

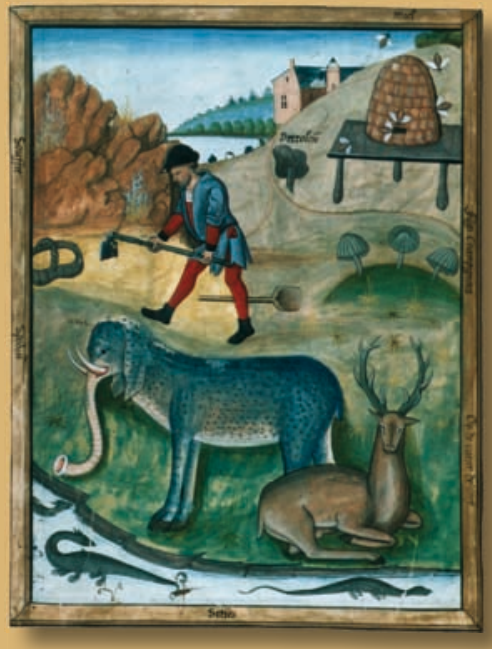



Manfred Jakubowski-Tiessen (Hg.)

Von Amtsgärten und Vogelkojen

Dieses Werk ist lizenziert unter einer Creative Commons Namensnennung Weitergabe unter gleichen Bedingungen 4.0 International Lizenz.

(2) (1) () 
erschienen im Universitätsverlag Göttingen 2014 
Manfred Jakubowski-Tiessen (Hg.)

Von Amtsgärten und

Vogelkojen

Beiträge zum Göttinger

Umwelthistorischen Kolloquium

2011-2012



Universitätsverlag Göttingen

2014 


\title{
Bibliographische Information der Deutschen Nationalbibliothek
}

Die Deutsche Nationalbibliothek verzeichnet diese Publikation in der Deutschen Nationalbibliographie; detaillierte bibliographische Daten sind im Internet über $<$ http://dnb.ddb.de $>$ abrufbar.

Die Veröffentlichung dieser Aufsatzsammlung dokumentiert Aktivitäten des DFG Graduiertenkollegs 1024 „Interdisziplinäre Umweltgeschichte. Naturale Umwelt und gesellschaftliches Handeln in Mitteleuropa“, in dessen Veranstaltungskanon das Umwelthistorische Kolloquium seit 2004 integriert ist.

Gedruckt mit Unterstützung der Deutschen Forschungsgemeinschaft

\author{
Anschrift des Graduiertenkollegs \\ Graduiertenkolleg 1024 \\ Interdisziplinäre Umweltgeschichte \\ Naturale Umwelt und gesellschaftliches Handeln in Mitteleuropa \\ Georg August Universität Göttingen \\ Bürgerstr. 50 \\ 37073 Göttingen \\ URL http://www.anthro.uni-goettingen.de/gk/
}

Dieses Buch ist auch als freie Onlineversion über die Homepage des Verlags sowie über den OPAC der Niedersächsischen Staats- und Universitätsbibliothek

(http://www.sub.uni-goettingen.de) erreichbar.

Es gelten die Lizenzbestimmungen der Onlineversion.

Redaktion: Jana Sprenger

Satz und Layout: Arne Ulrich, Martin Wiegand

Umschlaggestaltung: Kilian Klapp und Maren Büttner

Titelabbildung: Titelbild unter freundlich genehmigter Verwendung einer Abbildung aus MS 12322 Bibliothèque Nationale Paris, Section des Manuscriptes Occidentaux. 


\section{Bereits erschienen:}

\section{Bernd Herrmann (Hg.) 2004-2010/2011 \\ Beiträge zum Göttinger \\ Umwelthistorischen Kolloquium}

2004-2006

Universitätsverlag Göttingen 2007

als online-Version unter

http://webdoc.sub.gwdg.de/univerlag/2007/umweltkolloquium.pdf

2007-2008

Universitätsverlag Göttingen 2008

als online-Version unter

http://webdoc.sub.gwdg.de/univerlag/2008/umweltkolloquium_2.pdf

2008-2009

Universitätsverlag Göttingen 2009

als online-Version unter

http://webdoc.sub.gwdg.de/univerlag/2009/umweltkolloquium3.pdf

2009-2010

Universitätsverlag Göttingen 2010

Als online-Version unter

http://webdoc.sub.gwdg.de/univerlag/2010/umweltkolloquium4.pdf

2010-2011

Universitätsverlag Göttingen 2011

Als online-Version unter

http://webdoc.sub.gwdg.de/univerlag/2011/Umweltkolloquium5.pdf 



\section{Vorwort}

\section{Manfred Jakuboreski-Tiessen}

Im vorliegenden Band sind Vorträge gesammelt, welche mit einer Ausnahme alle im Göttinger Umweltgeschichtlichen Kolloquium im Wintersemester 2011/12 und im Sommersemester 2012 gehalten wurden. Das Umweltgeschichtliche Kolloquium, eine seit etwa dreißig Jahren kontinuierlich bestehende Einrichtung an der Göttinger Universität, ist seit dem Jahre 2004 organisatorisch aufs engste mit dem Graduiertenkolleg „Interdisziplinäre Umweltgeschichte“ verbunden gewesen.

Mit dem vorliegenden sechsten Band der Beiträge zum Göttinger Umwelthistorischen Kolloquium wird wiederum die thematische Vielfalt umweltgeschichtlicher Forschungen dokumentiert, wie diese von Anfang an das Profil des Kolloquiums bildete. ${ }^{1}$ Der interdisziplinären Ausrichtung des Graduiertenkollegs entsprechend sind die Beiträge unterschiedlichen wissenschaftlichen Ansätzen verpflichtet. Gerade die Interdisziplinarität hat die Forschungsvorhaben und Diskussionsforen der Göttinger Umweltgeschichte stets ausgezeichnet. Die in diesem Band gesammelten Beiträge behandeln umweltgeschichtliche Themen unterschiedlicher historischer Epochen; zeitlich erstrecken sich die Themen vom Mittelalter bis ins 20. Jahrhundert. Die Aufsätze widmen sich der Wahrnehmungsgeschichte von Natur und Naturkatastrophen in der frühen Neuzeit, der Geschichte des Naturraums Wattenmeer, Aspekten der Agrargeschichte und der Gartenkultur, der Frage der Wiederverwertung von Sachgütern im 18. Jahrhundert sowie der transnationalen Umweltgeschichte und den internationalen Beziehungen der Umweltbewegung.

\footnotetext{
${ }^{1}$ Die bereits erschienenen fünf Bände der Beiträge zum Göttinger Umwelthistorischen Kolloquium (2004 - 2011) wurden von Bernd Herrmann herausgegeben.
} 
Da das von der DFG geförderte Graduiertenkolleg „Interdisziplinäre Umweltgeschichte" nach neunjährigem erfolgreichem Wirken im Jahr 2013 ausgelaufen ist, hat auch das Umwelthistorische Kolloquium sein Ende gefunden. Es ist jedoch zu hoffen, dass die Umweltgeschichte auch künftig ihren Ort an der Georg-AugustUniversität in Göttingen haben wird.

Für die organisatorische Hilfe bei der Durchführung des Umwelthistorischen Kolloquiums danke ich den beiden wissenschaftlichen Hilfskräften Arne Ulrich und Martin Wiegand. Letzterer hat stets dafür gesorgt, dass die Veranstaltungen rechtzeitig angekündigt wurden und die technische Einrichtung im Hörsaal stets funktionsfähig war. Zu danken habe ich auch Dr. Lars Fehren-Schmitz, jetzt Professor an der University of California in Santa Cruz, der uns freundlicherweise für die Vorgespräche zum Kolloquium einen Raum mit einer ausgesprochen bizarren Kulisse im Johann-Friedrich-Blumenbach-Institut für Zoologie und Anthropologie zur Verfügung gestellt hat. 


\section{Inhaltsverzeichnis}

„The Wisdom of God“"

Das Erdbeben von Jamaica 1692 im Kontext von John Rays Physikotheologie

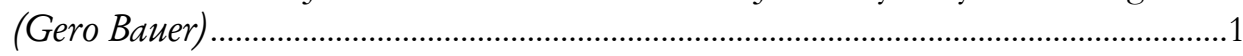

Die Gärten der Amtshäuser.

Förderung der Gartenkultur im 18. Jahrhundert am Beispiel der Gärten der Amtshäuser im Kurfürstentum Hannover (Jens Beck)

Nutz, Pflicht und Vergnügen:

Umweltwahrnehmungen im europäischen Landwirtschaftsschrifttum des 16. und 17. Jahrhunderts (Philip Hahn)

Interactions between the Australian and German Environmental Movements (Astrid Mignon Kirchhof) 67

Transnationale Umweltgeschichte (Patrick Kupper) 79

Naturgeschichte Wattenmeer (Karsten Reise) 91 
Der Kojenmann

Mensch und Natur im Wattenmeer, 1860-1900

(Martin Rheinheimer).

Agrarinnovationen in Mittelalter und Neuzeit

(Werner Rösener)

Weiternutzen, Reparieren, Wiederverwerten.

Der „Umgang mit den Dingen“ in der Vormoderne

(Georg Stöger).

Autoren 


\title{
„The Wisdom of God“" Das Erdbeben von Jamaica 1692 im Kontext von John Rays Physikotheologie ${ }^{1}$
}

\author{
Gero Bawer
}



1 Dieser Aufsatz ist ein überarbeiteter Auszug aus meiner Zulassungsarbeit: Bauer G (2009) Strafge-
richt Gottes oder Teil der Ordnung. Frühneuzeitliche Erdbebenliteratur im Kontext der physikotheo-
logischen Bewegung des 17. und 18. Jahrhunderts, Zulassungsarbeit. Tübingen. In dieser Arbeit stelle
ich die Schriften Rays und Kants vergleichend gegenüber und skizziere die Entwicklung des Verhält-
nisses von Religion und Naturwissenschaft von der Antike bis ins 18. Jahrhundert.
2 Kant I (1910) Von den Ursachen der Erderschütterungen bei Gelegenheit des Unglücks, welches
die westlichen Länder von Europa gegen das Ende des vorigen Jahres betroffen hat. In: Königlich
Preußische Akademie der Wissenschaften (ed) Kants gesammelte Schriften. Band I: Vorkritische
Schriften I. 1747-1756. Berlin. S. 417-427. Hier S. 419.
3 Eifert C (2002) Das Erdbeben von Lissabon 1755. Zur Historizität einer Naturkatastrophe. In:
Historische Zeitschrift 274. München. S. 633-664. Hier: S. 633ff.; Löffler U (1999) Lissabons Fall - 
mittelbarer zeitlicher Nähe zum Erdbeben entstanden Gedichte und Predigten, später ganze Abhandlungen; jeder wollte das Naturereignis kommentieren und Erklärungsversuche anbieten ${ }^{4}$. Am bekanntesten ist wohl Voltaires bissige Satire auf den populären Optimismus seiner Zeit, „Candide“, in der Voltaire das Erdbeben von Lissabon dazu benutzt, um die Leibnizsche Vorstellung von der „besten aller möglichen Welten“" ad absurdum zu führen ${ }^{5}$.

Die Prominenz des Lissabonner Erdbebens und seine ausführliche Rezeption sollten jedoch nicht das Bewusstsein dafür verdrängen, dass es auch andere Naturereignisse in der Frühen Neuzeit gibt, die die Aufmerksamkeit der Geschichtsforschung verdienen. Gerade bei der Beschäftigung mit Naturkatastrophen lohnt ein Blick über das 18. Jahrhundert (und über die Grenzen Europas) hinaus und zurück in das 17. Jahrhundert.

Schon 1692 erschien in dem Werk „Miscellaneous Discourses Concerning the Dissolution and Changes of the World" ${ }^{\text {" }}$ des britischen Botanikers und Universalgelehrten John Ray eine ausführliche Abhandlung über das Erdbeben von Jamaika im Jahr 1692, das eine vergleichbare Reaktion bei den Zeitgenossen hervorrief wie das Erdbeben von Lissabon 7 . Schon hier findet sich ein Diskurs im Spannungsfeld zwischen einer Interpretation der Naturkatastrophe als göttliches Strafgericht und einer den aufkommenden modernen Wissenschaften entsprechenden Erklärung des Phänomens als natürlichen Vorgang.

Rays Schrift über das Erdbeben von Jamaika soll hier exemplarisch die These widerlegen, die aufkommenden Naturwissenschaften hätten die Religion im Verlauf der Frühen Neuzeit in einem linear verlaufenden Prozess als Instanz für Fragen nach der Entstehung der Welt und ihrer Einrichtung verdrängt. Im Gegenteil, im Verlauf des 17. Jahrhunderts entstand mit der Physikotheologie eine einflussreiche ideengeschichtliche Strömung, die zum ersten Mal in der Geschichte den Versuch unternahm, Religion und Naturwissenschaft miteinander zu verbinden und nicht als zwei separate Sphären zu betrachten, wie es seit der Antike der Fall gewesen war. Dass dies vor allem deshalb geschah, weil die wissenschaftliche Revolution des 17. Jahrhunderts religiöse Weltanschauungen massiv ins Wanken brachte und sich die Naturwissenschaften im Zuge der Aufklärung in der westlichen Welt endgültig gegen die Dominanz religiös-christlicher Weltbilder durchsetzten, soll dabei nicht bestritten werden. Tatsache ist aber, dass es oft gerade diejenigen waren, die - wie John Ray - Pionierarbeiten in den Naturwissenschaften leisteten, die

Europas Schrecken. Die Deutung des Erdbebens von Lissabon im deutschsprachigen Protestantismus des 18. Jahrhunderts. De Gruyter, Berlin, New York. S. 1f.

${ }^{4}$ Löffler (1999), S. 2f.

${ }^{5}$ Eifert (2002), S. 634.

${ }^{6}$ Ray J (1692) Miscellaneous Discourses Concerning the Dissolution and Changes of the World. Wherein The Primitive Chaos and Creation, the General Deluge, Fountains, Formed Stones, SeaShells found in the Earth, Subterraneous Trees, Mountains, Earthquakes, Vulcanoes, the Universal Conflagration and Future State, are largely Discussed and Examined. London.

${ }^{7}$ Schnurmann C (2001) Das Erdbeben von Jamaika (Juni 1692) im zeitgenössischen Verständnis des englischen Kolonialreichs. Katastrophen als Mittel der Weltdeutung. In: P. Münch (ed) „Erfahrung“ als Kategorie der Frühneuzeitgeschichte. München. S. 249-259. 
am stärksten versuchten, traditionelle theistische und christliche Vorstellungen in die Moderne zu retten. Der Physikotheologie als Hybriddisziplin zwischen Naturwissenschaft und Theologie kommt dabei eine Schlüsselrolle zu.

Anhand des Beispiels Ray soll gezeigt werden, dass Naturkatastrophen wie Erdbeben die sehr populäre Physikotheologie des 17. und 18. Jahrhunderts vor eine große Herausforderung stellten. Schon Ray bemüht sich um eine für seine Zeit extrem fortschrittliche naturwissenschaftliche Betrachtung von Erdbeben. In dieser Hinsicht steht er Immanuel Kant, der mehr als 60 Jahre später seine Schriften verfasste, in nichts nach. Aber nicht Ray, der die Physikotheologie mit begründete, sondern Kant, der diese Denkrichtung später als nicht beweiskräftig verurteilen sollte, war der erste, dem es gelang, ein scheinbares Übel wie das Erdbeben von Lissabon mit der Idee einer wohlwollend geordneten Schöpfung in Einklang zu bringen. Ray scheiterte noch an dieser Herausforderung.

War einerseits die Frühe Neuzeit nicht - wie oft angenommen - der Abschied von der Religiosität, sondern eher eine Hochzeit der Verbundenheit von Wissenschaft und Theologie, so waren andererseits die Entwicklungen während dieser Periode in sich ein sehr komplexer Vorgang.

Sicher ist, dass während der Frühen Neuzeit ein Prozess in Gang kam, der die Aufklärung und eine zunehmende Säkularisierung der Welt mit sich brachte. Dass dies aber ein Prozess war, der alles andere als linear verlief, soll hier gezeigt werden.

\section{Begriffsdefinition „Physikotheologie“}

Eine Beschäftigung mit der Physikotheologie bringt die Schwierigkeit mit sich, dass in der Forschung kein Konsens darüber besteht, was genau unter diesem Begriff verstanden wird. Besonders die englischsprachige Literatur lässt oft Grenzen zwischen Begrifflichkeiten verschwimmen und vermeidet eine explizite Definition des Diskussionsgegenstandes. Viele Autoren verwenden die Begriffe „physicotheology“ und „natural theology“ synonym ${ }^{8}$ oder benennen die ,physico-theology“ überhaupt nicht, obwohl das Thema implizit angesprochen wird ${ }^{9}$. Andere - und in diesem Fall auch deutsche - Autoren, die den Begriff „Physikotheologie“ teilweise

\footnotetext{
8 So z. B. Brooke JH (1991) Science and Religion. Some Historical Perspectives. Cambridge Univ. Press, Cambridge. Besonders Kapitel VI „The Fortunes and Functions of Natural Theology“, S. 192225. Obwohl Brooke hier klar das Phänomen der Physikotheologie im teleologischen Sinn behandelt, nennt er den Begriff selbst im gesamten Kapitel nur einmal (S. 197), ohne näher auf ihn einzugehen. 9 So z. B. Barbour IG (1997) Religion and Science. Historical and Contemporary Issues. SCM, London. Er verwendet den Ausdruck „physico-theology“ nicht, obwohl er in seiner Beschreibung der „natural theology“ explizit „the argument from design“ (z. B. S. 20), also das Argument des teleologischen Gottesbeweises, thematisiert; auch Olson RG (2004) Science and Religion, 1450-1900. From Copernicus to Darwin. Greenwood Press, Westport, nennt „the argument from design“ (z. B. S. 108) im Zusammenhang seiner Diskussion der „,natural theology“, ohne auf die „physico-theology“ einzugehen.
} 
sogar im Titel ihrer Abhandlungen führen, verwenden den Ausdruck zwar kontinuierlich, allerdings ohne ihn vorher einzugrenzen und zu definieren ${ }^{10}$.

Aufgrund dieser unklaren Verwendung von Begrifflichkeiten ist es wichtig, vor einer Diskussion der Entwicklung der Physikotheologie den Begriff näher zu bestimmen. Wolfgang Philipp hat in seiner theologiegeschichtlichen Studie zur Aufklärung nicht weniger als acht Möglichkeiten aufgezeigt, wie Physikotheologie im weiteren und engeren Sinn in der - vor allem deutschsprachigen - Literatur definiert werden konnte, von einer Gleichsetzung mit dem System des Philosophen Christian Wolff über die Bezeichnung für verschiedene philosophische Schulen und unterschiedliche Periodisierungen bis hin zu geistesgeschichtlichen Vorgängen, einer Gleichsetzung mit dem scholastischen Verfahren der Theologia Naturalis und schließlich einer Bewegung, die „den ,Erweis“ Gottes vor den Wundern der Schöpfung unternimmt "11. Diese letzte Möglichkeit - die Physikotheologie als Bewegung, die versuchte, Gott aus den Wundern der Schöpfung zu beweisen - entspricht der am meisten verbreiteten Verwendung des Begriffs „Physikotheologie“.

Der Ausdruck selbst kommt in England Mitte des 17. Jahrhunderts auf und wird zum ersten Mal explizit von Walter Charleton in seiner Abhandlung „The darkness of atheism dispelled by the light of nature“12 mit dem Untertitel „A physico-theological treatise " verwendet ${ }^{13}$. Stefan Lorenz versteht unter Physikotheologie „die teleologische Betrachtung der Körperwelt und den Beweis, der von der so konstatierten zweckmäßigen Einrichtung, Vollkommenheit und Schönheit dieser Welt auf die Existenz Gottes und seine Eigenschaften schließt $t^{\text {“14 }}$.

Udo Krolzig definiert die Pysikotheologie in eben diesem Sinn. Er unterscheidet zwischen der theologischen Bewegung der Physikotheologie und der theologischen Argumentationsfigur, die aus dieser hervorging. Die theologische Argumentationsfigur stellt den „Schluß von einer vollkommenen, sinnvollen und schönen Ordnung des Universums auf einen allmächtigen, allweisen und gütigen Baumeister" ${ }^{\prime 15}$ dar. Diese sehr allgemeine Form des teleologischen Gottesbeweises ist natürlich keine Erfindung der Neuzeit; die Argumentationsform findet sich bereits in der Antike bei Sokrates und wird bis in die Gegenwart von Autoren herangezo-

\footnotetext{
${ }^{10}$ So z. B. Büttner M (1995) Wechselseitige Beziehungen zwischen Theologie und Naturwissenschaft (insbesondere Klimatologie) im 18. Jahrhundert. Physikotheologie als praktische natürliche Theologie. In: M. Büttner, F. Richter (ed) Forschungen zur Physikotheologie im Aufbruch I. Naturwissenschaft, Theologie und Musik in der Aufklärung. Referate des Symposiums in Halle. Münster. S. 3-49.

11 Philipp W (1957) Das Werden der Aufklärung in theologiegeschichtlicher Sicht. Vandenhoeck u. Ruprecht, Göttingen. S. 16.

${ }^{12}$ Charleton W (1652) The darkness of atheism dispelled by the light of nature. A physico-theological treatise. London.

${ }_{13}$ Lorenz S (1989) Physikotheologie. In: Historisches Wörterbuch der Philosophie, Band 7. Basel. Sp. 948-955. Hier: Sp. 948; Harrison P (2005) Physico-Theology and the Mixed Sciences. The Role of Theology in Early Modern Natural Philosophy. In: P. R. Anstey, J. A. Schuster (ed) The Science of Nature in the Seventeenth Century. Patterns of Change in Early Modern Natural Philosophy. Dordrecht. S. 165-183. Hier S. 172.

${ }^{14}$ Lorenz (1989), Sp. 948.

${ }^{15}$ Krolzig U (2003) Physikotheologie. In: H. D. Betz (ed) Religion in Geschichte und Gegenwart, Band 6. Mohr Siebeck, Tübingen. Sp. 1328-1330. Hier Sp. 1328.
} 
gen ${ }^{16}$. Die theologische Bewegung lässt sich allerdings zeitlich weiter eingrenzen. Sie beschreibt eine praktische Theologie der Natur und eine in diesem Zusammenhang entstandene eigene Gattung theologischer Literatur, die, ausgehend von England, in der zweiten Hälfte des 17. Jahrhunderts entstand, und in der ,aufgrund der Betrachtung von zweckmäßig, geordnet oder auch nur schön erscheinenden Strukturen oder Prozessen in der Natur auf das Dasein und die Eigenschaften Gottes, insbesondere seine Allmacht, Weisheit und Güte verwiesen wird" 17.

Es ist wichtig, darauf hinzuweisen, dass die physikotheologische Literatur den Gottesbeweis auf die neuesten naturwissenschaftlichen Erkenntnisse stützt, die meist kompendienhaft zusammengetragen werden und den theologischen Gedanken vorangestellt werden ${ }^{18}$.

Udo Krolzig hat zu Recht betont, dass die physikotheologischen Werke des 17. und 18. Jahrhunderts eine eigene Gattung theologischer Literatur darstellen. Obwohl sie oft mit Apologien verglichen werden, fehlt es ihnen an charakteristischen dialogischen Merkmalen, da Apologien sich immer an ein Gegenüber, beispielsweise an die Atheisten, wenden. Dieses Gegenüber ist in den physikotheologischen Schriften meist nicht vorhanden ${ }^{19}$. Sowohl Krolzig als auch Philipp weisen auf die wichtige Abgrenzung von Physikotheologie und Theologia Naturalis hin. Allerdings widerspricht Krolzig dem Einwand Philipps, die Theologia Naturalis schließe auf dem Weg apriorischer Vernunftschlüsse auf Gott, während die Physikotheologie „a posteriori aus der Fülle der Welt auf Gott" schließe ${ }^{20}$, da fast alle scholastischen Gottesbeweise a priori die Existenz der Welt und ihre Kontingenz annehmen ${ }^{21}$. Den Unterschied sieht Krolzig vielmehr im neuartigen Interesse der Physikotheologen an naturwissenschaftlichen Detailstudien, an denen die klassische Theologia Naturalis nicht interessiert war ${ }^{22}$. Tatsächlich wird unten deutlich werden, dass physikotheologische Werke wie die von John Ray oft zum Großteil rein naturwissenschaftliche Kompendien waren und die theologische Reflexion nur einen kleinen Teil des Gesamtwerks einnahm. Krolzig argumentiert weiter, physikotheologische Arbeiten seien auch keine Erbauungsbücher, da sie den Leser zwar zum Staunen vor der Natur aufriefen, dies aber aufgrund detaillierter, kausalmechanischer Untersuchungen geschehe, nicht unter Hinweis auf natursymbolische Zusammenhänge ${ }^{23}$.

Einer der wenigen englischsprachigen Autoren, der sich die Mühe macht, Begrifflichkeiten zu definieren, ist Neal Gillespie. Er weist darauf hin, dass der Ausdruck „,natural theology“ sich meist allgemein auf „theological beliefs drawn from

16 Krolzig U (1996) Physikotheologie. In: Theologische Realenzyklopädie, Band 26. Berlin. S. 590-

596. Hier: S. 591.

${ }^{17}$ Krolzig (1996), S. 591.

${ }^{18}$ Ebd.; Krolzig (2003), Sp. 1329; Lorenz (1989), Sp. $948 f$.

19 Krolzig U (1988) Säkularisierung der Natur. Providentia-Dei-Lehre und Naturverständnis der Frühaufklärung. Neukirchener Verl., Neukirchen-Vluyn. S. 150f.

20 Philipp (1957), S. 72.

${ }^{21}$ Krolzig (1988), S. 151.

22 Ebd.

${ }^{23}$ Ebd., S. 152. 
the interpretation of nature“ bezieht und „physico-theology“ eine Unterkategorie der „natural theology“ sei, „which emphasizes adaptive design in nature directed toward the accomplishment of purposeful ends"24. Obwohl Gillespie selbst diese Unterscheidung konsequent anwendet, gebrauchen die meisten englischsprachigen Autoren, wie oben erwähnt, beide Begriffe ohne klar ersichtliche Unterscheidung.

Hier soll Physikotheologie die geistesgeschichtliche Strömung und ihre Literatur bezeichnen, die im 17. Jahrhundert im Zusammenhang mit dem Aufkommen der modernen Wissenschaft und als Reaktion auf die damit verbundene Entmystifizierung der Natur in England aufkam und anhand neuester naturwissenschaftlicher Erkenntnisse, Beobachtungen und Experimente die Wunder und Schönheit der Natur demonstrierte und davon auf die Existenz, Güte und Weisheit Gottes schloss.

\section{2 „The Wisdom of God“ - John Rays Physikotheologie}

John Ray ${ }^{25}$ war sich bewusst, dass sein physikotheologisches Hauptwerk „The Wisdom of God Manifested in the Works of the Creation" keine völlig neuartige Idee vorbrachte. In seinem Vorwort nennt er einige Namen zeitgenössischer Physikotheologen und entschuldigt sich sogar dafür, sein Buch könne als „superfluous Piece $^{\text {" } 26}$ angesehen werden. Trotzdem rechtfertigt er die Veröffentlichung mit drei Gründen: Erstens hoffe er, sein Buch enthalte ,some Considerations new and untoucht by others“27; zweitens sei es ,more suitable to some Mens Apprehension, and facile to their Understandings“" 28 als andere Werke; und drittens seien die in diesem Buch enthaltenen Informationen in dieser Weise in keinem anderen einzelnen Buch zu finden, sondern ,lye scattered and dispersed in many“"29, was Rays

\footnotetext{
${ }^{24}$ Gillespie NC (1987) Natural History, Natural Theology, and Social Order. John Ray and the "Newtonian Ideology". In: Journal of the History of Biology 20 (1987). Kluwer, Dordrecht. S. 1-49. Hier S. 4.

25 Obwohl John Ray in England als Gründer der modernen Biologie angesehen und in seiner Bedeutung für die Wissenschaften mit Robert Boyle verglichen wird, ist er in der Geschichtswissenschaft bisher noch nicht angemessen gewürdigt worden. Die einzige Monographie zu Rays Leben und Werk von Charles E. Raven erschien bereits 1942 (Raven CE [1942] John Ray, Naturalist. His Life and Works. Cambridge Univ. Press, Cambridge.) und wurde in den folgenden Jahren von nur einer Studie wesentlich ergänzt, nämlich von Geoffrey Keynes' ausführlicher Bibliographie von Rays Werken (Keynes G [1951] John Ray. A Bibliography. Faber u. Faber, London.). Ansonsten erschienen nur einige weitere Aufsätze zu Einzelaspekten, die sich im Wesentlichen auf Raven stützen (z. B. Crowther JG [1960] Founders of British Science. John Wilkins, Robert Boyle, John Ray, Christopher Wren, Robert Hooke, Isaac Newton. Cresset Press, London. Oder: Arber A [1943] „A SeventeenthCentury Naturalist: John Ray. In: Isis 34.4 [1943]. S. 319-324). Diese wenig ausführliche Beschäftigung mit dem Leben Rays ist umso verwunderlicher, da die große physikotheologische Schrift Rays Ray J (1691) The Wisdom of God Manifested in the Works of the Creation. Being the Substance of Some Common Places Delivered in the Chappel of Trinity-College, in Cambridge. London. - in fast jeder größeren Bibliothek zu finden ist.

${ }^{26}$ Ray (1691), [,Preface“. Eigene Zählung: S. I].

${ }^{27}$ Ebd.

${ }^{28}$ Ebd. [,Preface“, Eigene Zählung: S. II].

${ }^{29}$ Ebd.
} 
Buch sowohl zu einer kompakten als auch preislich günstigen Alternative für interessierte Käufer mache.

„The Wisdom of God“ basiert auf Predigten, die Ray früh in seiner Karriere in Cambridge gehalten hatte. Das Werk besteht aus einer Abhandlung über fast alle Dinge der Natur und des Universums, von den Himmelskörpern und den Grundelementen der Erde über Pflanzen und Tiere bis hin zu der Beschaffenheit der Erde selbst und dem menschlichen Körper mit seinen einzelnen Organen ${ }^{30}$. Das Neue in diesem Buch im Verhältnis zu Rays früheren Werken ist die Abkehr vom reinen Identifizieren, Beschreiben und Klassifizieren zu einer Interpretation der beobachtbaren Prozesse in der Natur ${ }^{31}$. Das Buch ist eine Art Katalog von Wundern, der den Leser davon überzeugen soll, dass nur eine göttliche Intelligenz eine solch erstaunliche Ordnung geschaffen haben kann. Rays Beschreibung der Natur ist für James G. Crowther ,,a brilliant re-statement of the argument for the existence of God from the evidence of design, in the terms of the latest science of his day“"32. In diesem Vorhaben sah sich Ray bestärkt durch das erwachende Interesse vieler Laien an den Naturwissenschaften und dem ebenso großen Bedürfnis vieler Theologen, die neuen Erkenntnisse mit der Religion in Einklang zu bringen. Das große Verdienst von „The Wisdom of God“ ist sicherlich nicht, das physikotheologische Argument zum ersten Mal vorgebracht zu haben, aber Ray verband seine eigenen weitreichenden Erfahrungen in der Feldforschung und sein großes Wissen mit der Vorarbeit anderer Autoren und machte das physikotheologische Argument einer breiten Leserschaft zugänglich ${ }^{33}$. Gerade seine große Sorgfalt und Erfahrung als Naturwissenschaftler trugen ohne Zweifel maßgeblich zum Erfolg seines physikotheologischen Werks bei.

Ray selbst macht gleich zu Anfang seines Buchs sein Vorhaben deutlich. Er stellt dem Haupttext den biblischen Psalm 104, Vers 24 voran: „How Manifold are thy Works O Lord? In Wisdom hast thou made them all." ${ }^{34}$ Daraufhin erläutert er, dass er diese beiden biblischen Aussagen in seinem Buch beweisen will, wobei der Hauptteil des Buchs der zweiten Aussage, also der weisen Einrichtung der Schöpfung, gewidmet sein soll ${ }^{35}$.

Ray bekennt sich ausdrücklich zur „Mechanical Hypothesis“36, also zur These, die Entstehung des Universums sei auf rein naturgesetzliche Vorgänge zurückzuführen und diese Entstehung beruhe auf von Gott geschaffenen mechanischen Gesetzen. Daraufhin formuliert Ray in eigenen Worten das ,argument from design“:

\footnotetext{
30 Ray (1691), [,The Contents"]

31 Raven (1942), S. 452f.

32 Crowther (1960), S. 125.

33 Gillespie (1987), S. 39.

34 Ray (1691), S. 1.

35 Ebd., S. 1-11. Ray geht auf die Vielseitigkeit und für den Menschen unbegreifbare Menge an unterschiedlichen Lebewesen und unbelebten Dingen in der Natur ein.

36 Ebd., S. 11.
} 
„There is no greater, at least no more palpable and convincing Argument of the Existence of a Deity than the admirable Art and Wisdom that discovers itself in the make and constitution, the order and disposition, the ends and uses of all the parts and members of this fabrick of Heaven and Earth. ${ }^{\text {“37 }}$

Dabei zieht Ray den gleichen Analogieschluss, der Kant später als zentraler Ansatzpunkt seiner Kritik an der Physikotheologie dienen sollte:

„For if in the works of Art, as for example: a curious Edifice or Machine, counsel, design, and direction to an end appearing in the whole frame and in all the several pieces of it, do necessarily infer the being and operation of some intelligent Architect or Engineer, why shall not also in the Works of Nature, that Grandeur and Magnificence, that excellent contrivance for Beauty, Order, Use, \&c. which is observable in them, wherein they do as much transcend the Effects of human Art as infinite Power and Wisdom exceeds finite, infer the existence and efficiency of an Omnipotent and All-wise Creator? ‘38

Ray schließt aus der sinnvollen Ordnung und der Schönheit der Natur auf einen allmächtigen und weisen Schöpfer, weil der Vergleich mit menschlichen Kunstwerken diesen Analogieschluss nahe legt. Diese Analogie ist typisch für Vertreter einer mechanischen Weltsicht, die das Universum gern mit einem Uhrwerk verglichen.

Obwohl Ray selbstverständlich von einer traditionellen Weltsicht geprägt war, war er erstaunlich frei von Schranken in seinem Denken. Eine mechanische Weltanschauung widersprach für ihn nicht seinem Glauben, denn er war davon überzeugt, dass die kompromisslose Anwendung der Vernunft sowohl in der Wissenschaft als auch im Glauben der richtige Weg sei und es keine bessere Möglichkeit gebe, Gott zu loben, als nach der Wahrheit in Gottes Schöpfung zu forschen ${ }^{39}$.

Interessant ist vor allem, dass Ray hier eine rein deistische Position endgültig ablehnt. Er geht explizit auf Descartes' These einer rein mechanischen Welt ein, die die Rolle Gottes auf den Schöpfungsakt reduziert ${ }^{40}$, und widerspricht ihr mit der Begründung, dass erstens aus der Tatsache, dass es Bewegung in der Natur gibt, nicht folge, dass diese Bewegung sich immer von allein fortsetzt, sondern eine bewegungserhaltende Kraft vorausgesetzt werden müsse; zweitens Materie an sich verstandes- und vernunftlos sei und somit auch keinen äußerlichen Gesetzen folgen könne. Daraus folgert Ray, es müsse neben Materie und Naturgesetz einen Vermittler geben, der entweder eine der Materie innewohnende Eigenschaft, Gott oder eine „Plastick Nature“ sein müsse. Er entscheidet sich für die „Plastick Nature" als wahrscheinlichste Hypothese ${ }^{41}$.

\footnotetext{
37 Ebd., S. $11 \mathrm{f}$.

38 Ebd., S. 12.

${ }^{39}$ Raven (1942), S. 454f.

${ }^{40}$ Ray (1691), S. 32.

${ }^{41}$ Ebd., S. 33f.
} 
Ray geht es vor allem darum, die Funktionen der einzelnen Elemente der Natur für die Welt als Ganzes und insbesondere für den Menschen aufzuzeigen. Er betont beispielsweise, dass auch diejenigen Dinge, die der Mensch sich erst selbst erschaffen muss, im Grunde von Gott schon für den menschlichen Nutzen bereitet worden seien.

„For whether we think it or no, it is, for Example, manifest, that Fuel is good to continue Fire, and Fire to melt Metals, and Metals to make Instruments to build Ships and Houses, and so on. Wherefore it being true, that there is such a subordinate usefulnes in the Things themselves that are made to our Hand, it is but reason to impute it to such a Cause as was aware of the usefulnes and serviceablenes of its own Works. “42

Rays „Wisdom of God“ spiegelt die aktuellsten intellektuellen Strömungen seiner Zeit wider. Er verarbeitet nicht nur neueste naturwissenschaftliche Erkenntnisse und eine beispiellose Vielzahl an eigenen Beobachtungen, sondern ist auch in seinen theologischen Reflexionen ein Vordenker bei der Verbindung von Religion und Naturwissenschaft in einer Zeit des Umbruchs.

\section{Das Erdbeben von Jamaika 1692}

Die Frühe Neuzeit war nicht nur eine Zeit des wissenschaftlichen Fortschritts, sondern auch die Zeit der Entdeckungsreisen und der europäischen Kolonisierung der Welt. Für die Wahrnehmung von Erdbeben, um die es hier gehen soll, spielte dieser Umstand eine zentrale Rolle, denn die vielen Schriftzeugnisse, die europäische Reisende in ihre Heimat schickten, enthielten Berichte über Abenteuer und Wunder exotischer Welten und eben auch Beschreibungen von Erdbeben, die in bestimmten Regionen der Welt verstärkt auftraten. Meist handelt es sich um Augenzeugenberichte, die relativ schlichte Beschreibungen der Geschehnisse enthalten, denn zu diesen „exotischen“ Katastrophen, die nicht die eigene Heimat betrafen, bestand eine gewisse innere Distan $z^{43}$.

Allerdings waren die ökonomischen Folgen dieser Katastrophen manchmal enorm, denn nicht selten waren europäische Kolonien betroffen, die wichtige Rohstoffe abwarfen ${ }^{44}$.

Ein solches folgenreiches Ereignis war auch das Erdbeben von Jamaika im Juni 1692, das die englische Wirtschaft direkt betraf. England hatte sich im Zuge der Restauration schnell von einem landwirtschaftlich geprägten Land zu einer großen Handelsnation entwickelt. Im Zuge dieser Entwicklung wurde von den Monarchen

\footnotetext{
42 Ebd., S. $112 f$.

43 Vermij RK (2003) Erschütterung und Bewältigung. Erdbebenkatastrophen in der Frühen Neuzeit. In: M. Jakubowski-Tiessen, H. Lehmann (ed) Um Himmels Willen. Religion in Katastrophenzeiten. Vandenhoeck u. Ruprecht, Göttingen. S. 235-237. Hier: S. 242.

44 Ebd.
} 
auch die britische Kolonisierung fremder Länder verstärkt betrieben. Wilhelm III. ließ nach einigen nicht-permanenten Vorläuferorganisationen den Board of Trade einrichten, der den Handel in den Kolonien und der restlichen Welt fördern sollte. Die Kolonien in exotischen Regionen der Welt waren besonders wichtig für den englischen Handel, da sie Güter abwarfen, die in Europa nicht zu haben und daher begehrt waren. Ein Schwerpunkt der englischen Kolonialmacht waren die karibischen Stützpunkte, besonders Jamaika, das England in den 1650er-Jahren den Spaniern abgerungen hatte, und das 1670 formell in englischen Besitz übergegangen war $^{45}$.

Am 17. Juni $1692^{46}$ wurde Jamaika von einem starken Erdbeben erschüttert. Das Beben zerstörte etwa 780 Häuser, und die Hafenstadt Port Royal, die Hauptstadt der englischen Kolonie, lag in Trümmern. Auf das Beben folgte eine zerstörerische Flutwelle, die zusammen mit Erdverschiebungen im Landesinneren große Teile der Zuckerrohrplantagen der Insel zerstörte. Bei der Katastrophe starben zeitgenössischen Quellen zufolge zwischen 1.500 und 3.000 Menschen während des Hauptbebens und vermutlich weitere 3.000 bei Nachbeben und durch Folgeschäden wie einstürzende Häuser, Überschwemmungen und Seuchen. Dieses Erdbeben hinterließ mit seinen verhältnismäßig hohen Opferzahlen und den negativen Folgen für die englische Wirtschaft einen bleibenden Eindruck bei den Zeitgenossen und wurde zu einem Element britischer Erfahrung ${ }^{47}$.

Die Nachricht von dem Beben verbreitete sich für damalige Verhältnisse sehr rasch. Bereits am 21. August, also gute zwei Monate nach dem Beben, veröffentlichte die „London Gazette“ eine Nachricht über das Beben, ohne jedoch eine moralische Wertung vorzunehmen. Als aber etwas später auch private Berichte aus Jamaika in England eintrafen, traten zu der neutralen Berichterstattung auch sensationslustige und parteiische Schilderungen hinzu ${ }^{48}$. Ein Beispiel hierfür soll unten behandelt werden.

Die Wahrnehmung des Erdbebens von Jamaika lässt sich nach drei Gesichtspunkten gliedern: die Wahrnehmung der direkt Betroffenen, das Verständnis des Bebens als Strafe Gottes und das Beben als Objekt einer naturwissenschaftlich ausgerichteten Physikotheologie ${ }^{49}$.

Es sind einige Augenzeugenberichte des Erdbebens überliefert, die sich fast alle durch eine auffallende Nüchternheit der Beschreibung auszeichnen. Das Unglück wird lediglich beschrieben, Reflexionen über eine mögliche Schuld fehlen völlig. Die koloniale Leitung versuchte, die Folgen des Unglücks möglichst rasch

\footnotetext{
45 Browning A (1966) Trade and Plantations. Introduction. In: A. Browning (ed) English Historical Documents, Band VI: 1660-1714. Eyre u. Spottiswoode, London. S. 527-529. Hier: S. $527 \mathrm{f}$.

46 Die Datumsangabe folgt dem Gregorianischen Kalender, die Daten in den Quellen meist dem Julianischen Kalender. In dem „Anonymous account“, S. 567, datiert der Geistliche das Beben auf den 7. Juni.

47 Schnurmann (2001), S. $249 f$.

48 Ebd., S. 254.

${ }^{49}$ Ebd., S. 251-259.
} 
zu beseitigen und beschloss, Port Royal nicht wieder aufzubauen. Stattdessen entstand auf der anderen Seite der Insel die neue Hafenstadt Kingstown ${ }^{50}$.

Anders sieht die Schilderung eines englischen Geistlichen aus, der in Port Royal stationiert war. Sein Bericht, der auch Ray in seiner Behandlung des Bebens als Hauptquelle diente, repräsentiert die traditionelle Interpretation des Erdbebens als Strafe Gottes für die Sündhaftigkeit der Menschen. Das Beben sei ein ,terrible judgement of God" "51, die Port Royal selbst herausgefordert habe. Der Geistliche grenzt sich bewusst von den Bewohnern der Stadt ab, indem er betont, seine Aufgabe als Pfarrer habe darin bestanden, ,to keep up some show of religion amongst

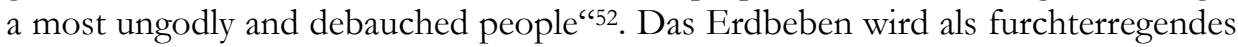
Ereignis beschrieben, das der Pfarrer hautnah miterlebte. „As I made towards it [Morgan's Fort] I saw the earth open and swallow up a multitude of people, and the sea mounting in over the fortification. " 53

Wie so oft bei Naturkatastrophen suchen auch die Menschen in Jamaika Schutz in der Religion. Der Pfarrer, der sich selbst in seinem Bericht zu einer Art Heilsfigur stilisiert, die vom Tod verschont wurde, beschreibt, wie die Menschen in ihrer Furcht zu ihm strömen. „Every one laid hold on my clothes and embraced me. [...] I prayed with them, [...] setting before them their sins and heinous provocations, and [...] seriously exhorting them to repentance. "54 Er tut zwar seine Pflicht und nutzt die Katastrophe, um die Menschen auf ihre Sündhaftigkeit hinzuweisen, fühlt sich aber auch abgestoßen, denn ,the people being so desperately wicked, makes me afraid to stay in the place“"55. Hier spricht der Geistliche ein weiteres Phänomen an, das Katastrophen oft begleitete. Die Menschen zeigen im Angesicht des Erdbebens ihre schlimmsten Seiten, rauben einander aus oder trinken und feiern, um die Angst zu vergessen.

Der Pfarrer betont besonders die ursprüngliche Schönheit und Größe Port Royals, die von Gott als Strafe für die sündhaften Bewohner zerstört wurde. Port Royal,

„the fairest town of all the English plantations, best emporium and mart of that part of the world, exceeding in its riches, plentiful in all good things, was shaken and shattered to pieces, sunk into and covered for the greatest part by the sea, and will in a short time be wholly eaten up by it. " 56

Eine ganz ähnliche Argumentation wird auch in der protestantischen Polemik nach dem Erdbeben von Lissabon wieder auftauchen.

\footnotetext{
${ }^{50}$ Ebd., S. 251.

51 o. N. (o. J.) A Full Account of the Late Dreadful Earthquake at Port Royal in Jamaica, Written in Two Letters From the Minister of That Place from Aboard the Granada in Port Royal. In: A. Browning (ed) (1966) English Historical Documents, Band VI: 1660-1714. Eyre u. Spottiswoode, London. S. 567-569. Hier: S. 567.

52 Ebd.

${ }^{53}$ Ebd., S. 568.

${ }^{54}$ Ebd.

${ }^{55}$ Ebd.

${ }^{56}$ Ebd., S. 569.
} 
Das Fazit des Pfarrers ist die Hoffnung, dass die Menschen aus dieser Strafe Gottes ihre Lehren gezogen haben. „I hope by this terrible judgement God will make them reform their lives, for there was not a more ungodly people upon the face of the whole earth. " 57

Der Bericht des englischen Geistlichen macht einerseits deutlich, wie Erdbeben im Kontext traditioneller Vorstellungen als Strafe eines durch die Natur strafenden Gottes gedeutet werden konnten, und offenbart andererseits ,alle unterschwelligen Abneigungen, Unsicherheiten und Vorurteile eines eingefleischten Engländers gegenüber der jungen, andersartigen Gesellschaft einer tropischen Pflanzerkolonie“"58. Jamaika war in der Vorstellungswelt der Engländer ,anders“, ein Ort außerhalb englischer Moralvorstellungen. Nicht zuletzt wurde das tropische Klima mit dem Überhandnehmen sexueller Leidenschaften assoziiert. Die außereuropäische Welt war in den Augen der Kolonialmacht minderwertig, und es herrschte die - natürlich im Grunde völlig den Tatsachen entgegenlaufende - Vorstellung vor, dass Erdbeben nur in exotischen Ländern vorkommen ${ }^{59}$.

Jäh widerlegt wurde diese Annahme im Kontext des Erdbebens von Jamaika, als am 18. September 1692, also im selben Jahr wie in der Kolonie auch, ein Erdbeben den Süden Englands erschütterte. Dieses Ereignis reduzierte die Distanz zwischen den Betroffenen des exotischen Bebens in Jamaika und den Bewohnern Englands. Es führte in England nicht nur zu einem verstärkten Interesse an dem Phänomen Erdbeben, sondern ließ viele Menschen auch die schnelle Bewertung des Bebens als Strafe Gottes für unmoralisches Verhalten überdenken. „Die persönliche Erdbebenerfahrung begünstigte die Verbindung von naturwissenschaftlichem Erkenntnisdrang und protestantischem Bekehrungsstreben in England. “60

Als Beispiel für die physikotheologische Herangehensweise an Erdbeben führt Claudia Schnurmann John Rays Reflexion über Erdbeben allgemein und das von Jamaika im Speziellen in seinen „Three Physico-Theological Discourses“ an. Im Folgenden soll untersucht werden, inwiefern Rays Abhandlung tatsächlich als Teil seiner Physikotheologie angesehen werden kann.

\section{Erdbeben in Rays „Three Physico-Theological Discourses“}

\subsection{Entstehungsgeschichte von Rays Buch}

Der Großteil von Rays wissenschaftlichen Werken war der Botanik und Zoologie gewidmet. Dabei beschränkte er sich im Wesentlichen auf die - ohne Frage für die spätere wissenschaftliche Biologie grundlegende - Aufgabe, Pflanzen und Tiere zu sammeln, zu benennen und zu katalogisieren. Diese Arbeit ist nicht die Art von

\footnotetext{
57 Ebd.

58 Schnurmann (2001), S. 253.

${ }^{59}$ Ebd.

60 Ebd., S. 255.
} 
Wissenschaft, die kontroverse Fragen aufwirft. Rays Naturgeschichte ließ sich relativ problemlos mit dem zu seiner Zeit immer noch sehr wörtlich interpretierten Bericht der Genesis in Einklang bringen. Erst als Ray anfing, sich auch mit Fragen der Geologie und Steinfunden auseinanderzusetzen, betrat er unsicheres Terrain. Ray beschäftigte sich schon früh mit Geologie. Dabei interessierten ihn besonders Fossilienfunde, deren versteinerte Formen ihn so sehr an die von ihm erforschten Pflanzen und Tiere erinnerten, dass er es für höchst unwahrscheinlich hielt, dass diese Ähnlichkeit nur Zufall sei. Entgegen der Meinung vieler seiner Zeitgenossen war Ray der Ansicht, diese Fossilien müssten einmal lebendig gewesen sein ${ }^{61}$.

Verstreuten Anmerkungen in seinen Briefen und kleineren Abhandlungen zu dem Thema lässt sich entnehmen, dass für Ray die größte Schwierigkeit darin bestand, fossile Funde, ihre geographische Verteilung und seine Überzeugung, es habe sich einst um lebendige Wesen gehandelt, mit der Bibel zu vereinbaren. Denn frühe Geologen waren hauptsächlich mit zwei Schwierigkeiten konfrontiert: mit der vorherrschenden Annahme, Gott habe die Welt genau so erschaffen, wie sie heute existiert, und der sich daraus ableitenden Folgerung, dass Arten nicht einfach aussterben konnten. Dass Ray bereit war, diese beiden Annahmen in Frage zu stellen, zeugt von seinem fortschrittlichen und aufgeklärten Geist, auch wenn er sich selbst nie völlig von biblischen Annahmen befreien konnte ${ }^{62}$.

Ein Problemfeld, das sich bei der Beschäftigung mit Geologie in der Frühen Neuzeit auch öffnete, war die Frage nach dem Alter und der Entstehung der Erde. Obwohl schon die astronomischen Erkenntnisse Keplers und Galileis das alte Weltbild ins Wanken gebracht hatten, sorgte erst das Aufkommen einer modernen Geologie für ernste Probleme und warf Fragen nach dem Alter der Erde und besonders nach der Dauer und den Auswirkungen der biblischen Sintflut auf. Wissenschaftler und Theologen waren wieder einmal mit der Aufgabe konfrontiert, zwischen neuen Erkenntnisse und orthodoxen Weltvorstellungen zu vermitteln ${ }^{63}$.

Auch Ray beschloss, sich zu der Problematik zu äußern. Ermutigt vom Erfolg seines Buchs „The Wisdom of God“ und dem breiten Interesse an dem Thema veröffentlichte er im Jahr 1692 seine „Miscellaneous Discourses concerning the Dissolution and Changes of the World“. In diesem Werk behandelt Ray verstärkt geologische Phänomene unter physikotheologischen Gesichtspunkten. Der Aufbau orientiert sich an der biblischen Schöpfungsgeschichte. Nacheinander werden die Entstehung der Welt, die Sintflut, ihre - aus damaliger Sicht - bis heute feststellbaren Auswirkungen und die Zukunft der Welt betrachtet ${ }^{64}$. Ray geht es darum, anhand seiner Beobachtungen sein Argument für natürliche Prozesse innerhalb einer von Gott geschaffenen Gesetzmäßigkeit zu demonstrieren. Dabei will er den biblischen Bericht nicht widerlegen, sondern nimmt ihn als Grundlage und kombiniert ihn mit den wissenschaftlichen Erkenntnissen seiner Zeit. „If, with Ray, they

${ }^{61}$ Raven (1942), S. $419 f$.

${ }^{62}$ Ebd., S. 424-429.

${ }^{63}$ Ebd., S. $429 f$.

${ }^{64}$ Keynes (1951), S. 110. 
[scientists] questioned points like ,the novity of the earth' or the effects of the Flood, they were still prepared to draw their evidence from authority or from observation almost indiscriminately and to regard the field of knowledge as a whole." 65

Schon 1693 erschien die zweite, stark erweiterte Auflage des Buchs, nun unter dem neuen Titel „Three Physico-Theological Discourses“66. Besonders wichtig für die hier behandelte Fragestellung ist, dass Ray in diesem Werk einen Abschnitt den Erdbeben und ihrem Zweck und Nutzen für die Welt widmet. Dieser Teil enthält auch ausführliche Beschreibungen des Erdbebens von Jamaika, das erst kurz zuvor stattgefunden hatte, und des kurz darauf folgenden englischen Erdbebens ${ }^{67}$. Ob es Ray gelingt, das physikotheologische Argument auf das Phänomen der Erbeben anzuwenden und naturwissenschaftliche und theologische Betrachtungen angemessen zu verbinden, soll in den folgenden Kapiteln untersucht werden.

Rays „Discourses“ erreichten nicht die Popularität seines „The Wisdom of God“, aber neben drei weiteren Auflagen nach Rays Tod in den Jahren $1713^{68}$, $1721^{69}$ und $1732^{70}$ mit Kommentaren von William Derham, erschienen $1694{ }^{71}$ und $1783^{72}$ niederländische und $1698^{73}$ und $1732^{74}$ deutsche Übersetzungen des Buchs, was vor allem für die Rezeption Rays bei den deutschen Physikotheologen von zentraler Bedeutung ist.

\footnotetext{
65 Raven (1942), S. 441.

${ }^{66}$ Ray J (1693) Three Physico-Theological Discourses, Concerning I. The Primitive Chaos, and the Creation of the World. II. The General Deluge, its Causes and Effects. III. The Dissolution of the World, and Future Conflagration. Wherein Are Largely Discussed the Production and Use of Mountains; the Original Fountains, of Formed Stones, and Sea Fishes Bones and Shells found in the Earth; the Effects of particular Floods and Inundations of the Sea; the Eruptions of Vulcano's; the Nature and Causes of Earthquakes: With an Historical Account of those Two late Remarkable Ones in Jamaica and England. With Practical Inferences, [...], The Second Edition Corrected, very much Enlarged, and Illustrated with Copper-plates. Smith, London. Für alle Angaben zu Auflagen und Übersetzungen siehe Keynes (1951), S. 110-116.

${ }^{67}$ Raven (1942) S. 446f.; Keynes (1951), S. 111.

${ }^{68}$ Ray J (1713) Three Physico-Theological Discourses, [...] Wherein are largely discussed, [...] Also an Historical Account [...], The Third Edition, Illustrated with Copper-Plates, and much more Enlarged than the former Editions, from the Author's own MSS. Innys, London.

${ }^{69}$ Ray J (1721) Three Physico-Theological Discourses, [...], Illustrated with Copper Plates, [...], The Fourth Edition. Innys, London.

70 Ray J (1732) Three Physico-Theological Discourses, [...], The Fourth Edition Corrected. Innys, London. Obwohl hier „Fourth Edition“ angegeben ist, handelt es sich tatsächlich um die fünfte Auflage.

${ }^{71}$ Ray J (1694) De Werelt van haar Begin tot haar Einde. Barent Bos, Rotterdam.

${ }^{72}$ Ray J (1783) De Wereld van haar Begin tot haar Eynde. In Drie Natuurkundige Godgeleerde Redeneringen. Rakende I. Den eersten Choas, en de Scheppinge der Wereld. II. Den allgemeene Zundvloed deszelfs Oorzaaken en nitwerkingen. III. De ontflopinge van de Wereld en toekomende Verbrandinge, [...]. o. O.

${ }^{73}$ Ray J (1698) Sonderbares Klee-Blättlein, der Welt Anfang, Veränderung und Untergang [...]. Wiering, Hamburg.

${ }^{74}$ Ray J (1732) Drey Physico-Theologische Betrachtungen Von der Welt Anfang, Veränderung und Untergang. Worinnen I. Das erste Chaos, oder der unförmliche vermischte Klumpen, und die Erschaffung Himmels und der Erden, II. Die allgemeine Sündfluth, deren Ursachen und Würckungen, III. Die Auflösung der Welt und zukünfftige Verbrennung; Ins besondere aber die Herfürbringung und Nutzen der Berge, [...], Aus dem Englischen übersetzt Von Theodor Arnold. Löwe, Leipzig.
} 
Den folgenden Ausführungen liegt die Auflage von 1713 zugrunde, da sie als Reprint am besten zugänglich ist ${ }^{75}$. Der untersuchte Abschnitt ist Teil des zweiten der „Discourses“, in dem Ray sich mit der biblischen Sintflut und ihren Auswirkungen bis in seine Gegenwart beschäftigt ${ }^{76}$. Das fünfte Kapitel, in dem es um Veränderungen an der Erdoberfläche seit der Zeit der Sintflut geht ${ }^{77}$, behandelt verschiedene geologische Phänomene, unter anderem auch Erdbeben ${ }^{78}$.

\subsection{Versuch einer naturwissenschaftlichen Erklärung}

Rays Darstellung von Erdbeben besteht zum Großteil aus der nüchternen Beschreibung der Phänomene. Hier wird seine Vertrautheit mit dem Sammeln und Zusammenstellen von Informationen deutlich. Er beginnt seine Ausführungen mit dem Bezug auf antike Autoren, die selbst Erdbeben beschrieben haben. Interessanterweise nennt er seine Gewährsmänner „Naturalists and Historians“"79, schreibt also den antiken Autoren ein naturwissenschaftliches Interesse an Erdbeben zu. Ray nennt beispielsweise Poseidonios (135 v. Chr. - 51 v. Chr.) ${ }^{80}$, Strabo (ca. 63 v. Chr. - 23 n. Chr.) ${ }^{81}$, Duris (geb. ca. 330 v. Chr.) ${ }^{82}$, Demetrius Calatianus (3. Jhd. v. Chr.) ${ }^{83}$, und Plinius (ca. $23-79$ n. Chr.) ${ }^{84}$. Es scheint ihm vor allem darum zu gehen, möglichst viele konkrete Auswirkungen der Beben aus den antiken Beschreibungen zu entnehmen. Beispielsweise geht er darauf ein, dass Demetrius Calatianus beschreibt, wie die heißen Quellen von Aedepsus und Thermopylae nach einer Reihe von Erdbeben versiegten, um dann nach drei Tagen wieder zu fließen ${ }^{85}$. Außerdem versucht Ray, aus den überlieferten Beschreibungen und seiner eigenen Erfahrung allgemeine Schlüsse zu ziehen. So stellt er fest, dass in Neapel, Apulien und Kalabrien mehr Erdbeben vorkommen als im übrigen Europa ${ }^{86}$.

Ray geht ausführlich auf das kurz vor der Veröffentlichung seines Buches geschehene Erdbeben von Jamaika ein. Als Grundlage dient ihm der oben zitierte Bericht des anonymen englischen Pfarrers. Es ist auffällig, dass sich Ray, obwohl der Bericht des Geistlichen zum Großteil aus moralischen Anmerkungen besteht,

\footnotetext{
75 Ray J (1978) Three Physico-Theological Discourses. Reprint of the 1713 ed. New York.

${ }^{76}$ Ebd., S. 61-295: Discourse II: Of the general Deluge in the Days of Noah, its Causes and Effects.

77 Ebd., S. 206-295: Chap. V: That there have been great Changes made in the Superficial Part of the Earth since the General Deluge, and by what Means.

78 Ebd., S. 246-282.

${ }^{79}$ Ebd., S. 246.

80 Weiterführend siehe Malitz J (1983) Die Historien des Poseidonios. Beck, München.

81 Weiterführend siehe Prontera F (ed) (1984/1986) Strabone. Contributi allo studio della personalitá e dell'opera, 2 Teile. Perugia.

82 Weiterführend siehe Kebric RB (1977) In the shadow of Macedon. Duris of Samos. Steiner, Wiesbaden.

$83 \mathrm{Zu}$ Calatianus findet sich kaum Literatur. Kruse FCH (1827) Hellas oder geographischeantiquarische Darstellung des alten Griechenlandes und seiner Colonien. Mit steter Rücksicht auf die neuen Entdeckungen. Voß, Leipzig, S. 135, erwähnt, dass Calatianus über die Erdbeben in Griechenland schrieb.

${ }^{84}$ Ray (1693), S. 246-249.

85 Ebd., S. 247.

${ }^{86}$ Ebd., S. 250.
} 
hauptsächlich auf die berichteten Tatsachen bezieht. Dabei kommentiert er sogar manche Passagen und bringt kritische Anmerkungen an. Die Aussage des Geistlichen, das Erdbeben habe Felsen und Berge auseinandergebrochen, ergänzt er durch weitere Quellen: „Others tell us, that it [the earthquake] leveled some Moun-

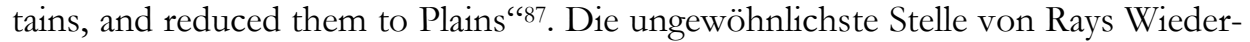
gabe des Augenzeugenberichts ist eine Anmerkung, die bis zu einem gewissen Grad von Rays Interesse an einer neutralen, wissenschaftsorientierten, nicht wertenden Sicht auf das Erdbeben zeugt. Er vermeidet die Wiedergabe der moralischen Verurteilung der Bevölkerung Port Royals, da sein Bericht nicht dazu diene, diese Menschen zu beschämen. „Then he [the Minister] acquaints us, what for to save the Reputation of the People, and to avoid the laying a perpetual Blot upon them, I should rather suppress and conceal." 88 Dass er dieses Vorhaben nicht konsequent durchhält, wird unten deutlich.

Eine weitere Ergänzung aus eigenem Wissen findet sich eine Seite später, wo Ray den Bericht des Geistlichen von dem verstärkten Vorkommen von Raubüberfällen nach dem Erdbeben durch den Hinweis ergänzt: „The like Robbers and Plunderers we were told wander'd up and down the Country, even in the very Smoke, during the last geat Burning and Eruption of Aetna in Sicily." ${ }^{\text {"69 }}$

Eine ähnliche Analogie zieht Ray im Zusammenhang mit dem konkreten Ablauf des jamaikanischen Erbebens. In der Bemerkung des Geistlichen, der Morgen vor der Erderschütterung sei sehr klar gewesen, sieht Ray ein allgemein verbreitetes Phänomen, da Ähnliches auch schon an anderen Orten beobachtet worden sei: "This is observed of most Earthquakes, and particularly of our last here in England, the Morning before it, being clear and calm. ${ }^{\text {"90 }}$ Ray überprüft also die Glaubwürdigkeit seiner Hauptquelle, indem er Berichte von anderen Erdbeben oder eigene ergänzende Beobachtungen hinzuzieht.

Nachdem Ray den Bericht des Geistlichen mit einigen persönlichen Anmerkungen wiedergegeben hat, beginnt der eigentliche Teil seiner Reflexionen über die Ursachen und den Ablauf von Erdbeben im Allgemeinen. In diesen Ausführungen beweist Ray ein breites Wissen der aktuellen Theorien über die Entstehung von Erdbeben und zeigt das Bestreben, dieses Naturphänomen mit nüchternem wissenschaftlichem Blick zu betrachten.

Ray glaubt, Erdbeben und Donner hätten die gleichen Ursachen, nämlich „Exhalations and Steams set on fire“"91. Diese Ausdünstungen und Dämpfe seien im Falle der Erdbeben in der Erde eingeschlossen. Was die Beschaffenheit der Dämpfe betrifft, bezieht sich Ray auf Lister ${ }^{92}$, der Schwefel für einen Hauptbestandteil entzündlicher Dämpfe halte. Die Frage für Ray ist, worauf die Entzünd-

\footnotetext{
87 Ebd., S. 251.

88 Ebd.

89 Ebd., S. 252.

90 Ebd., S. $252 \mathrm{f}$.

91 Ebd., S. 258.

92 Martin Lister (ca. 1638-1712), englischer Naturforscher und Arzt. Ray gibt nicht an, welchem Werk Listers er seine Informationen entnimmt.
} 
lichkeit der Dämpfe zurückzuführen ist, und er schließt - wiederum unter Bezugnahme auf Experimente Listers -, dass ein Gemisch aus Wasserdampf und Schwefel für die unterirdischen Entzündungen verantwortlich sei ${ }^{93}$. Ray vermutet nun, dass das entzündliche Gemisch unter der Erde entstehe und dann ,in manner of Explosion, like that of Gun-powder" ${ }^{(94}$ die Erde zum Beben bringe.

Um seine Vermutung zu untermauern, bringt Ray Beispiele für die Entzündlichkeit unterirdischer Dämpfe an, die er den Schriften Boyles und anderer Autoren entnommen hat. Diese Beispiele, die hauptsächlich Bergbauunfälle beschreiben, dienen Ray als Beweis für die verschiedenen Eigenschaften unterirdischer Dämpfe. Dazu zählten ihre leichte Entzündlichkeit, ihre Explosionskraft, die meist von einem lauten Knall begleitet werde, und ein beißender Geruch ${ }^{95}$. Aus all diesen Beispielen schließt Ray, dass ,this Damp should seem to be but Gun-powder in a Vapour, and to partake the Sulphur, Nitre, and Bitumen [...and] That our Earthquakes in Enlgand, and any others that have but one single Pulse, owe there Original to the Kindling and Explosion of Fire-Damps " ${ }^{\text {(66. }}$.

Um seine Theorie zu erhärten, geht Ray vorausschauend auf eventuellen Widerspruch ein. Dass bei Erdbeben selten tatsächlich Feuer sichtbar werde, liege an der Tiefe der Entzündung in der Erde, die sich zwar ihren Weg durch unterirdische Kanäle bahnen könne, aber nicht die Kraft habe, durch die Erdkruste zu brechen. Dies gelte auch für das jamaikanische Erdbeben, von dem keine Berichte über aus der Erde ausbrechende Feuer überliefert seien ${ }^{97}$.

Ein Zeichen für Rays wissenschaftlichen Anspruch ist auch seine Bescheidenheit in Fragen, für die er selbst kein Experte ist oder keine in seinen Augen ausreichende Quellengrundlage hat. Zu den genauen unterirdischen Abläufen der von ihm vorgestellten Entzündung bemerkt er beispielsweise: „But in this, I confess, I do not satisfy myself. They who have a more comprehensive Knowledge of all the Phaenomena, may give a better Account. " 98

Bemerkenswert ist besonders ein Hinweis im Zusammenhang mit dem Auftreten von Erdbeben beim Eintreten von Wasser in Vulkane. Hier bringt Ray das einzige $\mathrm{Mal}$ in seiner Behandlung von Erdbeben ein eigenes Experiment als Argument vor: „How great the Force of Water converted into Vapour is, I have sometimes experimented by inadvertently casting a Bullet in a wet Mold. “99

Zum Abschluss der Passage über Erdbeben bringt Ray als weiteres Beispiel das oben erwähnte englische Erdbeben. Für dieses Ereignis kann er sowohl auf eigene Erfahrung als auch auf eine große Menge von Augenzeugenberichten zurückgreifen. Dabei ist er sich bewusst, welchen Wert eine breite Sammlung empirischer Daten für die Erforschung der Eigenschaften des Bebens haben kann. Was

\footnotetext{
93 Ray (1693), S. 259 f.

94 Ebd., S. 261.

95 Ebd., S. 261-265.

96 Ebd., S. 265.

97 Ebd., S. 265-267.

98 Ebd., S. 268.

99 Ebd., S. 269.
} 
beispielsweise den Zeitpunkt der Erschütterung betrifft, bemerkt Ray: „Had we a punctual and exact Notice of the very Minute that it happened in far distant Places, we might thence gather something concerning the Motion and Progress of it."100 Ray bringt auch Informationen über die Wahrnehmung der Erschütterung im Ausland an. Dem Brief eines Freundes kann er entnehmen, dass das Erdbeben nicht nur in England zu spüren war, sondern sich auch auf andere Länder Europas ausdehnte. Er nennt „the Dutch and the Spanish Netherlands“"101, Deutschland und Frankreich. Besonders bemerkenswert sei, dass die Erschütterung vor allem in Küstenregionen zu spüren gewesen sei ${ }^{102}$.

Ray beschäftigt sich aber nicht nur mit den geologischen Auswirkungen des Bebens, sondern ist auch an dem Effekt auf die Menschen interessiert. Das Beben habe bei vielen ,a Swimming or Dizziness in their Heads“ verursacht und oft ,a Loathing and Inclination to Vomit" hervorgerufen, „All which must be the Effects, either of the Heaving, or tremulous Motion, or both; and yet, no Motion of Boat or Coach doth so suddenly affect and disturb the Head or Stomach“103.

Aus der Beschreibung des englischen Erdbebens zieht Ray den Schluss, dass sich die unterirdischen Höhlen, die die entzündlichen Dämpfe enthielten, unter dem Meer fortsetzen müssten, denn nur so sei die weite Ausstrahlung der Erschütterung zu erklären. Diese Hypothese sei auch „a great Confirmation of what we have before delivered concerning the Mountains of Aetna, Stromboli, and Vesuvius, communicating by submarine Vaults"104. Neueste Nachrichten von Beben in Flandern, Holland, Frankreich und Deutschland bekräftigten nach Ray die Theorie zusätzlich.

Rays Theorie von der Entstehung der Erdbeben beruht auf einer Fülle von Informationen, die entweder auf ihm verlässlich erscheinenden Quellen oder seiner eigenen Erfahrung basieren. Dabei bemüht er sich, seine Aussagen auf eine möglichst breite Informationsbasis zu stellen und ist sich auch bis zu einem gewissen Grad der Subjektivität seiner Quellen bewusst. Rays Vermutungen über die Ursachen von Erdbeben stellen einen Versuch dar, empirische Methode, die Aussagen antiker und zeitgenössischer Autoren und Aussagen von Augenzeugen zu einer kohärenten Theorie zusammenzuführen. Dabei ähnelt Rays Theorie in ihren Einzelheiten schon sehr den Aussagen von Wissenschaftlern des 18. Jahrhunderts wie beispielsweise Immanuel Kant ${ }^{105}$.

Es stellt sich die Frage, wie Ray den physikotheologischen Gedanken der Schönheit und Ordnung der Welt mit einem Phänomen wie dem der Erdbeben vereinbart.

\footnotetext{
100 Ebd., S. 272.

101 Ebd., S. 274.

102 Ebd., S. 273 f.

103 Ebd., S. 275.

104 Ebd., S. 276.

${ }^{105}$ Für eine Diskussion von Kants Erdbebentheorie siehe Bauer (2009), S. 74-82.
} 


\subsection{Die Rolle Gottes}

Konkrete theologische Reflexionen sind in Rays Ausführungen über die Erdbeben rar gesät. Doch schon die historischen Beispiele, die er zu Anfang des Abschnitts wählt, machen deutlich, dass Ray hier - wie überhaupt im gesamten Buch - in einem biblischen Kontext argumentiert. Bei einem Erdbeben im 6. Jahrhundert in Antiochien beispielsweise sei der dortige Bischof Gregor „by the Divine Favour, and in a manner miraculously preserved" ${ }^{\prime 106}$. Obwohl Ray generell von einem indirekten Einwirken Gottes in der Welt ausgeht und Naturphänomene anhand der Naturgesetze zu erklären versucht, scheint er sich nicht von traditionellen christlichen Wundervorstellungen lösen zu können.

Die Vorstellung von einem direkten Einwirken Gottes in der Welt durch Erdbeben wird auch deutlich, wenn Ray sich auf ein Beben im Jahr 1182 bezieht, „when Saladin set himself to overthrow the Kingdom of Jerusalem“107. Das Erdbeben wird hier zur Strafe Gottes für die Schändung der heiligen Stadt der Christen durch einen Ungläubigen.

Auch Rays Wiedergabe des Berichts des englischen Geistlichen über das Erdbeben von Jamaika ist - obwohl Ray doch, wie oben gezeigt, betont, Wertungen vermeiden zu wollen - nicht frei von theologischen Implikationen. Im Gegenteil: Gleich zu Beginn bezeichnet Ray das jamaikanische Beben als „terrible Judgement"108 und fühlt sich - obwohl er sich des Verstoßes gegen die Wissenschaftlichkeit seiner Ausführungen bewusst zu sein scheint - verpflichtet, die „Vindication of the Divine Providence and Justice"109, die in Gestalt des Erdbebens über Jamaika hereinbrach, dem Leser mitzuteilen, und zwar mit der Begründung, dass „,to deterr others from the like Enormities, I think necessary to publish, That the Inhabitants of that Place were a most ungodly and debauched People, and so desperately wicked, that he [the Minister] was even afraid to continue among them"110. An dieser Stelle beweist Ray das völlige Fehlen einer kritischen Haltung gegenüber seiner Quelle. Ohne an der Glaubwürdigkeit des Geistlichen in moralischen Fragen zu zweifeln, übernimmt Ray dessen Einschätzung der Bewohner Jamaikas, was vermutlich sowohl auf seinen Respekt gegenüber dem Geistlichen als auch auf zeitgenössische Wahrnehmungen exotischer Regionen wie Jamaika als Horte moralisch verwerflichen Verhaltens zurückzuführen ist.

Die Auswirkungen des Bebens auf die Überlebenden sieht Ray ebenfalls vollkommen im Kontext einer gleichsam alttestamentarischen Gottesstrafe, die die Menschen zum Umdenken bringen soll. „The Influence and Effect this Judgement had upon the Remainder of the People [was], to bring them to a Sense of their Sins, and Repentance for them, and to resolve upon, and begin a Reformation and

\footnotetext{
106 Ray (1693), S. 248.

107 Ebd., S. 249.

108 Ebd., S. 251.

109 Ebd., S. 252.

110 Ebd.
} 
Amendment of their Lives." "111 Interessanterweise schließt Ray seinen Kommentar des Wesens der Betroffenen mit einer Bemerkung ab, die nicht völlig in die sonst stark christlich geprägte Argumentation passt, sondern vielmehr eine allgemeine Beobachtung des Verhaltens von Menschen in Krisensituationen zu sein scheint:

„Fear is a more powerful Passion than Love: And whatever creates Terror, is a more effectual Curb to restrain and rule Men as well as Children, than any Favours or Benefits, the most powerful Motives of Love and Affection: For though the Bonds of Love are called the Cords of a Man, and are indeed very strong ones to rational and ingenuous Persons, yet the greatest part of Mankind are so far degenerated, that they have broken these Bonds, and cast these Cords from them; and upon Trial, one shall find little of Gratitude or Ingenuity among them. " ${ }^{112}$

Ray hat von seinen Zeitgenossen kein sehr positives Bild. Er sieht eine egoistische Menschheit. Erst durch die Erfahrung einer extremen Krisensituation wie eines Erdbebens, das Furcht in ihnen auslöst, werden sich die Menschen wieder gegenseitiger Liebe und Zuneigung bewusst.

Selbst in seiner stark von einer empirischen Herangehensweise geprägten Auflistung der konkreten Phänomene des Erdbebens ist Ray extrem von der biblischen Überlieferung beeinflusst, die er als ebenso verlässliche Quelle behandelt, wie den Bericht des Geistlichen. Zum Beispiel kam das Erdbeben von Jamaika für die Bewohner von Port Royal völlig unerwartet und ohne Warnung, „in which respect this Judgement resembled those on the Old World and on Sodom, which, the Scripture tells us, were, to the People involved in them, sudden and unexpected, and future Dissolution of the World by Fire, is predicted to be " ${ }^{\text {"113 }}$. Ray stellt das jamaikanische Erdbeben ganz in den Kontext göttlicher Strafgerichtsbarkeit und der Vorzeichen der Apokalypse.

Zentral in diesem Zusammenhang und für eine Interpretation des Textes im Kontext der Physikotheologie ist eine längere Ausführung Rays, in der er nochmals auf die moralische Verwerflichkeit der Bewohner Port Royals eingeht. Hier wird deutlich, dass es Ray weder gelingt, das Erdbeben unter rein naturgesetzlichen Gesichtspunkten zu betrachten, noch, das Unglück irgendwie sinnvoll in eine zeitgenössische physikotheologische Begründung einzubetten. Stattdessen fällt Ray in traditionell christliche Erklärungsmuster zurück. Zunächst stellt er allgemein fest, dass langer Frieden, Wohlstand und Reichtümer Stolz und Hang zu Luxus in einer Stadt oder einem Land hervorrufen können, was zu einem Verfall der Moral führe. $\mathrm{Zu}$ solchen Zeiten sende Gott sein Strafgericht, das die verdorbenen Menschen entweder zerstöre oder ihre Zahl zumindest stark verringere ${ }^{114}$. Als Beispiel für das bisher größte Gericht solcher Art bringt Ray wiederum biblische Beispiele an,

111 Ebd., S. 257.

112 Ebd., S. $257 f$.

113 Ebd., S. 258.

114 Ebd., S. 270. 
nämlich die große Sintflut und die Zerstörung von Sodom und Gomorrha. Was Ray dann tut, zeigt, dass er naturwissenschaftliche Methode und theologische Argumentation - ähnlich wie in seinen tatsächlich physikotheologischen Schriften nicht trennt: Er behauptet, historische Quellen zeigten, dass jeder größeren Katastrophe der moralische Verfall von Menschen voranging: „And we shall find it noted by Historians, That before any great publick Calamity, or utter Excision of a Nation, the People were become universally vicious and corrupt in their Manners, and without all Fear of GOD, or Sense of Goodness." 115 Bezeichnenderweise nennt Ray hier keine konkreten Gewährsmänner, und es ist nicht klar, ob seine Aussage zustande kommt, weil er wiederum biblische Überlieferungen als Quelle heranzieht, oder ob er schlicht eine unbegründete Behauptung aufstellt, um seine Position zu untermauern.

Was nun folgt, stellt Rays Unvermögen, das physikotheologische Argument auf Katastrophen zu übertragen, eindrucksvoll dar. Entgegen seiner Überzeugung, Gott wirke fast ausschließlich durch die Naturgesetze, glaubt er, im Erdbeben von Jamaika ein direktes Eingreifen Gottes zu sehen.

„For GOD doth not stand by as an idle and unconcerned Spectator, and suffer Things to run at Random, but his Providence many times interposes, and stops the usual Course and Current of Natural Causes: Nay, I believe and affirm, That in all great and Notable Revolutions and Mutations, He hath the greatest Hand and Interest; Himself ordering and governing them by His spezial Superintendance and Influence. So, though the Instruments and Materials wherewith this Devastation in $J a$ maica was made, as a subteraneous Fire and inflamable Materials, were before in the Earth, yet that they should at this time break forth and work, when there was such an Inundation of Wickedness there, and particularly and especially at Port-Royal, this we may confidently say, was the Finger of GOD, and effected perchance by the Ministery of Angels. “116

Mit einem Problem konfrontiert, das fast alle Physikotheologen schlicht außer Acht lassen, nämlich dem Problem des Übels in der Welt, sieht Ray sich nicht im Stande, ein Phänomen wie das Erdbeben von Jamaika mit dem physikotheologischen Argument, das Dasein Gottes äußere sich in der Schönheit und gesetzmäßigen Ordnung der Natur, in Einklang zu bringen. Stattdessen stellt er dieses Phänomen außerhalb der von Gott sinnvoll und wohlwollend geordneten Natur und interpretiert es als Strafe. Dabei scheint Ray außer Acht zu lassen, dass dies einerseits im Widerspruch steht zu der Vorstellung von einem gütigen Gott und außerdem zur von ihm selbst angeschlossenen Schilderung des englischen Erdbebens, in der er die Frage, warum nun auch in England Erdbeben stattfinden, wenn nicht als Strafe für moralische Verwerflichkeit, völlig außer Acht lässt ${ }^{117}$. Obwohl sich Ray der Theodizeedebatte nicht bewusst zu sein scheint, sie zumindest nicht explizit

115 Ebd., S. 271.

116 Ebd., S. 271.

117 Ebd., S. 272-281. 
thematisiert, ist es genau diese Problematik, an der seine Argumentation letztlich scheitert. Denn wie kann ein menschliches Unglück wie die Folgen eines Erdbebens - bei aller naturwissenschaftlichen Erklärung - im Kontext einer grundsätzlich von einem gütigen Gott ausgehenden Theorie wie der Physikotheologie sinnvoll erklärt werden?

Ray gelingt dieser Schritt nicht. Es wird erst Kant sein, der einen Versuch unternimmt, Erdbeben mit der Annahme der besten aller möglichen Welten in Einklang zu bringen, ohne dabei auf traditionelle Interpretationen der Katastrophe als göttliches Strafgericht zurückzugreifen ${ }^{118}$.

John Ray vertritt in vielen seiner Werke wissenschaftliche Prinzipien wie die exakte Beschreibung der Phänomene in der Natur und deren Systematisierung. Sein Anspruch ist, die Naturgesetzlichkeiten zu erkennen, in denen Gottes Vollkommenheit zum Ausdruck kommt. Insofern kann er als Vertreter der Aufklärung gelten. Bei seinem Erklärungsversuch einer Naturkatastrophe wie dem Erdbeben von Jamaika, durch die er mit dem immer wiederkehrenden Problem der Theodizeefrage konfrontiert wird, lässt Ray all diese vernünftigen Ansätze jedoch außer Acht und fällt in Erklärungsstrukturen der Voraufklärung zurück, indem er sich auf eine ausschließlich theologische Begründung des Bebens zurückzieht. Insofern steht Ray als Beispiel dafür, dass die Aufklärung und die Wendung hin zu vernünftigen Erklärungen von Naturphänomenen keine geradlinige Entwicklung darstellen.

\section{Literatur}

Arber A (1943) A Seventeenth-Century Naturalist: John Ray. In: Isis 34.4 (1943). S. 319-324.

Bauer G (2009) Strafgericht Gottes oder Teil der Ordnung. Frühneuzeitliche

Erdbebenliteratur im Kontext der physikotheologischen Bewegung des 17. und 18. Jahrhunderts, Zulassungsarbeit. Tübingen.

Barbour IG (1997) Religion and Science. Historical and Contemporary Issues. SCM, London.

Brooke JH (1991) Science and Religion. Some Historical Perspectives. Cambridge Univ. Press, Cambridge.

Browning A (1966) Trade and Plantations. Introduction. In: A. Browning (ed) English Historical Documents. Band VI: 1660-1714. Eyre u. Spottiswoode, London. S. 527-529.

Büttner M (1995) Wechselseitige Beziehungen zwischen Theologie und Naturwissenschaft (insbesondere Klimatologie) im 18. Jahrhundert. Physikotheologie als praktische natürliche Theologie. In: M. Büttner, F. Richter

118 Siehe Bauer (2009), S. 74-85. 
(ed) Forschungen zur Physikotheologie im Aufbruch I. Naturwissenschaft, Theologie und Musik in der Aufklärung. Referate des Symposiums in Halle. Münster. S. 3-49.

Charleton W (1652) The darkness of atheism dispelled by the light of nature. A physico-theological treatise. London.

Crowther JG (1960) Founders of British Science. John Wilkins, Robert Boyle, John Ray, Christopher Wren, Robert Hooke, Isaac Newton. Cresset Press, London.

Eifert C (2002) Das Erdbeben von Lissabon 1755. Zur Historizität einer

Naturkatastrophe. In: Historische Zeitschrift 274. München. S. 633-664.

Gillespie NC (1987) Natural History, Natural Theology, and Social Order. John

Ray and the "Newtonian Ideology". In: Journal of the History of Biology 20

(1987). Kluwer, Dordrecht. S. 1-49.

Harrison P (2005) Physico-Theology and the Mixed Sciences. The Role of

Theology in Early Modern Natural Philosophy. In: P. R. Anstey, J. A. Schuster (ed) The Science of Nature in the Seventeenth Century. Patterns of Change in Early Modern Natural Philosophy. Dordrecht. S. 165-183.

Kant I (1910) Von den Ursachen der Erderschütterungen bei Gelegenheit des Unglücks, welches die westlichen Länder von Europa gegen das Ende des vorigen Jahres betroffen hat. In: Königlich Preußischen Akademie der Wissenschaften (ed) Kants gesammelte Schriften. Band I: Vorkritische Schriften I. 1747-1756. Berlin. S. 417-427.

Kebric RB (1977) In the shadow of Macedon. Duris of Samos. Steiner, Wiesbaden. Keynes G (1951) John Ray. A Bibliography. Faber u. Faber, London.

Krolzig U (1988) Säkularisierung der Natur. Providentia-Dei-Lehre und Naturverständnis der Frühaufklärung. Neukirchener Verl., Neukirchen-Vluyn.

Krolzig U (1996) Physikotheologie. In: Theologische Realenzyklopädie, Band 26. Berlin. S. 590-596.

Krolzig U (2003) Physikotheologie. In: H. D. Betz (ed) Religion in Geschichte und Gegenwart, Band 6. Mohr Siebeck, Tübingen. Sp. 1328-1330.

Kruse FCH (1827) Hellas oder geographische-antiquarische Darstellung des alten Griechenlandes und seiner Colonien. Mit steter Rücksicht auf die neuen Entdeckungen. Voß, Leipzig.

Löffler U (1999) Lissabons Fall - Europas Schrecken. Die Deutung des Erdbebens von Lissabon im deutschsprachigen Protestantismus des 18. Jahrhunderts. De Gruyter, Berlin, New York. 
Lorenz S (1989) Physikotheologie. In: Historisches Wörterbuch der Philosophie, Band 7. Basel. Sp. 948-955.

Malitz J (1983) Die Historien des Poseidonios. Beck, München.

Olson RG (2004) Science and Religion, 1450-1900. From Copernicus to Darwin. Greenwood Press, Westport.

o. N. (o. J.) A Full Account of the Late Dreadful Earthquake at Port Royal in Jamaica, Written in Two Letters From the Minister of That Place from Aboard the Granada in Port Royal. In: A. Browning (ed) (1966) English Historical Documents, Band VI: 1660-1714. Eyre u. Spottiswoode, London. S. 567-569.

Philipp W (1957) Das Werden der Aufklärung in theologiegeschichtlicher Sicht. Vandenhoeck u. Ruprecht, Göttingen.

Prontera F (ed) (1984/1986) Strabone. Contributi allo studio della personalitá e dell'opera, 2 Teile. Perugia.

Raven CE (1942) John Ray, Naturalist. His Life and Works. Cambridge Univ. Press, Cambridge.

Ray J (1691) The Wisdom of God Manifested in the Works of the Creation. Being the Substance of Some Common Places Delivered in the Chappel of TrinityCollege, in Cambridge. London.

Ray J (1692) Miscellaneous Discourses Concerning the Dissolution and Changes of the World. Wherein The Primitive Chaos and Creation, the General Deluge, Fountains, Formed Stones, Sea-Shells found in the Earth, Subterraneous Trees, Mountains, Earthquakes, Vulcanoes, the Universal Conflagration and Future State, are largely Discussed and Examined. London.

Ray J (1693) Three Physico-Theological Discourses, Concerning I. The Primitive Chaos, and the Creation of the World. II. The General Deluge, its Causes and Effects. III. The Dissolution of the World, and Future Conflagration. Wherein Are Largely Discussed the Production and Use of Mountains; the Original Fountains, of Formed Stones, and Sea Fishes Bones and Shells found in the Earth; the Effects of particular Floods and Inundations of the Sea; the Eruptions of Vulcano's; the Nature and Causes of Earthquakes: With an Historical Account of those Two late Remarkable Ones in Jamaica and England. With Practical Inferences, [...], The Second Edition Corrected, very much Enlarged, and Illustrated with Copper-plates. Smith, London.

Ray J (1694) De Werelt van haar Begin tot haar Einde. Barent Bos, Rotterdam.

Ray J (1698) Sonderbares Klee-Blättlein, der Welt Anfang, Veränderung und Untergang [...]. Wiering, Hamburg. 
Ray J (1713) Three Physico-Theological Discourses, [...] Wherein are largely discussed, [...] Also an Historical Account [...], The Third Edition, Illustrated with Copper-Plates, and much more Enlarged than the former Editions, from the Author's own MSS. Innys, London.

Ray J (1721) Three Physico-Theological Discourses, [...], Illustrated with Copper Plates, [...], The Fourth Edition. Innys, London.

Ray J (1732) Drey Physico-Theologische Betrachtungen Von der Welt Anfang, Veränderung und Untergang. Worinnen I. Das erste Chaos, oder der unförmliche vermischte Klumpen, und die Erschaffung Himmels und der Erden, II. Die allgemeine Sündfluth, deren Ursachen und Würckungen, III. Die Auflösung der Welt und zukünfftige Verbrennung; Ins besondere aber die Herfürbringung und Nutzen der Berge, [...], Aus dem Englischen übersetzt Von Theodor Arnold. Löwe, Leipzig.

Ray J (1732) Three Physico-Theological Discourses, [...], The Fourth Edition Corrected. Innys, London.

Ray J (1783) De Wereld van haar Begin tot haar Eynde. In Drie Natuurkundige Godgeleerde Redeneringen. Rakende I. Den eersten Choas, en de Scheppinge der Wereld. II. Den allgemeene Zundvloed deszelfs Oorzaaken en nitwerkingen. III. De ontflopinge van de Wereld en toekomende Verbrandinge, [...]. o. O.

Ray J (1978) Three Physico-Theological Discourses. Reprint of the 1713 ed. New York.

Schnurmann C (2001) Das Erdbeben von Jamaika (Juni 1692) im zeitgenössischen Verständnis des englischen Kolonialreichs. Katastrophen als Mittel der Weltdeutung. In: P. Münch (ed) „Erfahrung“ als Kategorie der Frühneuzeitgeschichte. München. S. 249-259.

Vermij RK (2003) Erschütterung und Bewältigung. Erdbebenkatastrophen in der Frühen Neuzeit. In: M. Jakubowski-Tiessen, H. Lehmann (ed) Um Himmels Willen. Religion in Katastrophenzeiten. Vandenhoeck u. Ruprecht, Göttingen. S. 235-237. 



\title{
Die Gärten der Amtshäuser. Förderung der Gartenkultur im 18. Jahrhundert am Beispiel der Gärten der Amtshäuser im Kurfürstentum Hannover
}

\author{
Jens Beck
}

\section{Einleitung}

Die Geschichte der Gartenkultur ist trotz verstärkter Aktivitäten in den letzten Jahrzehnten ${ }^{1}$ noch in großen Teilen, vor allem hinsichtlich regionaler Besonderheiten, wenig erforscht. Zudem stehen bei den vorliegenden Arbeiten meist kunstgeschichtliche und künstlerische Fragen im Vordergrund ${ }^{2}$, während die Bedeutung der Gärten als Kulturträger im umfassenderen Sinne kaum thematisiert wird. Betrachtet man die Veröffentlichungen der jüngsten Zeit, die sich mit der Gartenkultur der vergangenen Jahrhunderte im niedersächsischen Raum befassen, so wird rasch deutlich, dass zwar die Zentren der Gartenkunst relativ gut untersucht worden sind und einige Arbeiten zu speziellen Fragestellungen vorliegen. Andere, ebenfalls interessante Themen sind jedoch bisher noch weitgehend unbeachtet geblieben, ja noch nicht einmal als solche formuliert worden. Dazu gehört die

\footnotetext{
${ }^{1}$ Bibliographie zur Gartenkunst/Gartengeschichte, seit 1987 bis 2001 erschienen in der Zeitschrift „Die Gartenkunst“, aktualisiert und fortgeführt online von der Pücklergesellschaft e.V. Berlin (http://www.pueckler-gesellschaft.de/haupt.html).

2 Buttlar A v. (2003) Der historische Garten als Gegenstand der wissenschaftlichen Forschung im 20. Jahrhundert. In: Vereinigung der Landesdenkmalpfleger in der Bundesrepublik Deutschland und Landesamt Berlin (ed) Historische Gärten. Berlin. S. 11ff.
} 
Gartenkultur in den ländlichen, abseits der kulturellen Mittelpunkte gelegenen Gebieten. Während beispielsweise die Geschichte der Gartenanlagen der kurfürstlichen und später königlichen Sommerresidenz in Hannover-Herrenhausen ${ }^{3}$ und die Anlagen der Oldenburger Großherzöge verhältnismäßig gut erforscht sind, gilt dies für die übrigen Residenzen - Celle, Braunschweig und Osnabrück - schon nicht mehr. Weitgehend unerforscht sind auch die zahlreichen Parkanlagen, die von den Familien des Landadels angelegt wurden. Immerhin wurde 2009 eine erste Dissertation über die Anlagen der Rittergüter im Elbe-Weser-Raum veröffentlicht. ${ }^{4}$ Dass auch die Ämter, die die untere Ebene der Verwaltung bildeten, über große und teilweise anspruchsvoll gestaltete Gärten verfügten, ist bislang nicht wahrgenommen worden. Dabei liegt gerade hier ein großes Forschungspotential, da die Ämter in der Regel eine sehr gute Archivierung betrieben haben und sich trotz einiger Verluste sehr viel Quellenmaterial erhalten hat.

Erstaunlich sind die beschriebenen Wissenslücken vor allem deshalb, weil eine der interessantesten Fragen diejenige nach der Ausbreitung der Gartenkultur ist. Wie wird die in den stilbildenden Anlagen entwickelte Gartenkunst im Land verbreitet? Auf welche Weise wird das Wissen über neueste Techniken des Obstbaus, wie werden neue Züchtungen weitergegeben? Wie funktioniert im Bereich der Gartenkultur der Prozess, der mit dem Begriff ,,absinkendes Kulturgut“ beschrieben wird? Auf anderen Gebieten der Kulturforschung, beispielsweise der Möbelkunst oder der Malerei liegen dazu teilweise ausführliche Untersuchungen vor, hier sind die Prozesse der Ausbreitung bestimmter Stilformen und -elemente bereits genauer dokumentiert worden.

Bezüglich der Gartenkunst müssen noch viele Punkte präzisiert und einige immer noch wiederholte Behauptungen korrigiert werden. Beispielsweise spielen entgegen der weit verbreiteten Meinung die Klöster für die Gartenkultur nach 1600 keine Rolle mehr. Denn sie sind spätestens durch die Entwicklungen nach dem 30jährigen Krieg als kulturelle Zentren marginalisiert. Die meisten wurden später zerstört, einige sind nur durch die Umwandlung in adlige Stifte erhalten geblieben. In einigen Gebieten verschwinden die Klöster vollständig, z. B. im Elbe-WeserRaum in den Jahren der schwedischen Besatzung.

Eine wichtige Rolle in den ländlichen Regionen kommt hingegen den Pastoren $\mathrm{zu}$, doch deren Wirken ist durch Quellen kaum belegt und daher nur schwer greifbar. Besser nachvollziehbar ist die Tätigkeit einiger Apotheker, doch beschränkte sich deren botanisches Engagement meist speziell auf die Heilpflanzen. Wesentlich für die Verbreitung der Gartenkultur im ländlichen Raum sind vor allem die beiden bereits genannten Gruppen von Anlagen: die Gärten der landadeligen Güter und die Gärten der Ämter. Der Erforschung der letztgenannten widmet sich ein von der DFG finanziertes Projekt, das an der TU Berlin derzeit bearbeitet wird.

\footnotetext{
${ }^{3}$ König M v. (2006) Herrenhausen. Die königlichen Gärten in Hannover. Wallstein, Göttingen.

${ }^{4}$ Beck J (2009) Historische Gutsgärten im Elbe-Weser-Raum. Dissertation an der Fakultät Architektur und Landschaft der Leibniz Universität Hannover. Stade.
} 


\section{Entwicklungsprogramme im 18. Jahrhundert für den ländlichen Raum}

Im 17. und 18. Jahrhundert wurde in vielen europäischen Staaten versucht, die lange vernachlässigten ländlichen Regionen durch bestimmte Entwicklungsprogramme zu stärken und den Lebensstandard der Bevölkerung zu heben, natürlich um so letztlich die Einnahmen des Staats über ein größeres Steueraufkommen zu erhöhen. Meist waren es Infrastrukturprogramme, beispielsweise der Wasser-, Deich- und Chausseebau, aber viele zielten auch auf die Verbesserung der Landund Forstwirtschaft, die Einführung des Tabak- und Seidenbaus, Förderung der Imkerei, Moorkolonisation etc.

In der Regierungszeit von Georg III. spielte die Förderung der Gartenkultur in all ihren Fassetten eine besondere Rolle. Dies lag zum einen an den eigenen Interessen des Königs, der in England den liebevollen Beinamen the farmer King oder farmer George erhielt. Georg III. war ein großartiger Förderer der Land- und Forstwirtschaft, er beschäftigte sich mit Schafzucht und Gartengestaltung, er interessierte sich für neue landwirtschaftliche Geräte und Anbaumethoden, er gründete die „Royal Horticultural Society of London“ und baute Kew Gardens zum bis heute führenden botanischen Institut aus. Im Stammland seiner Familie, das er bekanntlich nie besucht hat, schlug sich dieses Interesse dadurch nieder, dass er zumindest ähnliche Aktivitäten unterstützt und wohlwollend begleitet hat. In seiner Zeit wurden die Gartenanlagen in Herrenhausen weiter gepflegt und vergrößert.

Ein weiterer Beweggrund für die Förderung der Gartenkultur war die erhoffte Schaffung von neuen Erwerbszweigen, beispielsweise durch den Verkauf von Obst, was vor allem in der Nähe von größeren Städten ein lukratives Geschäft war. Auch in dem Verkauf von Gehölzen wurde eine mögliche Einnahmequelle gesehen. Darüber hinaus gab es Bestrebungen, die Ernährungsgewohnheiten der ländlichen Bevölkerung zu verändern und sie zu einer gesünderen Lebensweise zu animieren.

Aus diesen Gründen wurde vor allem der Verbreitung des Obstbaus eine besondere Bedeutung beigemessen. Im Kurfürstentum hatte sich jedoch - anders als in Preußen - die Erkenntnis durchgesetzt, dass bestimmte Entwicklungsziele nicht durch die Herausgabe einzelner Erlasse erreicht werden können, sondern nur durch die Schaffung bestimmter Rahmenbedingungen. Zur Förderung der Gartenkultur war es notwendig, zunächst einmal eine zentrale Produktionsstätte mit entsprechend ausgebildetem Personal zu schaffen. Georg III. ließ dazu gegenüber dem Sommerschloss ein regelrechtes Baumschulquartier anlegen. Neben der Vergrößerung des Berggartens gehörte dazu die Gründung der sog. Plantage 1767, einer Baumschule, in der in erster Linie Obstgehölze herangezogen wurden.

Hinzu kam ein im 19. Jahrhundert zunehmendes privates Engagement. 1832 wurde der „Gartenbauverein für das Königreich Hannover“ gegründet. Damit widmete sich neben der bereits 1797 gegründeten „Naturhistorischen Gesell- 
schaft" ${ }^{\text {“5 }}$ ein eigenständiger Verein dem Thema der „Hortikultur“. 6 Ziel der Mitglieder war wiederum die Förderung der Gartenkultur, vor allem auch des Obstbaus. Das Vorbild dieses Vereins und anderer ähnlicher war die bereits erwähnte „Royal Horticultural Society of London“, die 1804 gegründet wurde. Der bedeutendste Verein in Deutschland war der 1822 unter Mitwirkung von P. J. Lenné in Berlin gegründete „Verein zur Beförderung des Gartenbaues in den preußischen Staaten"7.

\section{Das wichtigste Zentrum der Gartenkultur in Norddeutschland: Hannover-Herrenhausen}

Die Sommerresidenz der welfischen Kurfürsten in Herrenhausen ist ein Beispiel für ein ganzes Ensemble hochwertiger Gärten mit jeweils eigenem Charakter und Funktion. Den Mittelpunkt bildet der ab 1666 aus der Umgestaltung einer unbedeutenden Vorgängeranlage entstandene Große Garten, der neben Salzdahlum bedeutendste Barockgarten des Kürfürstentums. Er ist durch die vierreihige Lindenallee, die über zwei Kilometer lange Große Allee, mit dem Stadtschloss verbunden. Entlang der Allee reihten sich verschiedene Gärten der adligen Landsitze auf, die im 19. Jahrhundert zum Georgengarten zusammengefasst wurden. Lediglich der Welfengarten, der aus dem früheren Park Monbrillant der Familie von Plate-Hallermund hervorgegangen ist, hat seine Eigenständigkeit bis heute bewahrt. Dem Großen Garten gegenüber lagen verschiedene Gärten, die nach 1850 dem Berggarten angegliedert wurden. Sie dienten in erster Linie der Anzucht verschiedener Gehölze und anderer Gewächse: der Maulbeergarten, in dem die für die Seidenproduktion notwendigen Maulbeerbäume herangezogen wurden; die Plantage, in der vorwiegend Obstgehölze veredelt und vermehrt wurden; und der Berggarten selbst, der aus einem früheren Küchengarten hervorgegangen ist. Hinzu kommt der Küchengarten in der damals noch selbständigen Stadt Linden, der in erster Linie der Versorgung des Hofes mit Gemüse diente.

Die genannten Anlagen prägen eine ganze Gegend, sie waren zumindest zeitweise öffentlich zugänglich und von wenigen Jahren der Vernachlässigung während der französischen Besetzung abgesehen in einem sehr guten Pflegezustand. So konnte sich der Besucher bereits im 18. Jahrhundert bei einem Spaziergang über nahezu alle Aspekte der Gartenkultur informieren. Sowohl Gartengestaltung wie auch neueste Züchtungen, Techniken des Gartenbaus, Gerätschaften, Gewächshäuser etc. konnten betrachtet und Pflanzen erworben werden. Herrenhau-

\footnotetext{
5 Gartenbauverein für das Königreich Hannover (1833) Verhandlungen des Gartenbauvereins für das Königreich Hannover. Heft 1. Hannover. S. 4.

${ }^{6}$ Die wechselvolle Geschichte des Vereins hat Benno Hülsmann in einer Festschrift 1982 dargestellt. Sehr ausführlich ist die Geschichte in einer Diplomarbeit von 2006 aufgearbeitet worden. Sablowski M (2006) Zur Geschichte des Gartenbauvereins der Hauptstadt Hannover e. V. 1832-2005. Hannover.

7 Zur Geschichte des Gartenbaus siehe Franz G (1984) Geschichte des deutschen Gartenbaus. Stuttgart.
} 
sen entwickelte sich dadurch im 18. Jahrhundert zu einem wichtigen Studienort für alle an der Gartenkultur interessierten Personen.

\section{Die Plantage in Herrenhausen}

Die als „Plantage“ bezeichnete Baumschule in Herrenhausen wurde 1767 als Produktionsstätte für Obstbäume gegründet. ${ }^{8}$ Sie zählte im 18. Jahrhundert zu den wichtigsten Einrichtungen ihrer Art in Europa. ${ }^{9}$ Durch die Personalunion zwischen England und Hannover besaß der niedersächsische Raum eine besonders enge Verbindung zum Mutterland der neuzeitlichen Gartenkultur. Nicht nur der Landschaftsgarten als völlige neue Form der Freiraumgestaltung konnte auf diese Weise zuerst im Stammland der englischen Könige Fuß fassen ${ }^{10}$, auch neueste Erkenntnisse auf dem Gebiet der Gartenkultur, neue Züchtungen und Importe aus Amerika und Asien wurden quasi auf kurzem Wege von England nach Hannover gebracht ${ }^{11}$. Der Plantage kommt eine Schlüsselstellung bei der Verbreitung der Gartenkultur in Deutschland zu, da sie einerseits als Verteilerzentrum fungierte, andererseits eine Schulungsstätte für zahlreiche Gärtner war. Der Kieler Philosophieprofessor Hirschfeld würdigte sie bereits 1782 als die ,,merkwürdigste öffentliche Baumschule in Deutschland“ und beschrieb ihre Aufgabe in seinem „Gartenkalender":

„Die eigene Vorsorge des Königs für die Wohlfahrt seiner Unterthanen hat die erste Grundlage zu diesem Institut gegeben. Er übersendete verschiedene Sämereien von amerikanischen Bäumen und Sträuchern, und befahl, Versuche damit zu machen, um ihre Kultur zu befördern. Man suchte die Absicht auch dadurch gemeinnütziger zu machen, daß man eine Obstbaumschule anlegte, welche durch Anziehung von weissen Maulbeerbäumen noch vermehret ward. [...] Von den Obstbäumen werden alle Jahr 2-3000 Stück den Unterthanen umsonst gegeben. Dieses geschiehet aber nicht willkührlich, sondern die königl. Kammer communiciert mit den Beamten. Diese müssen eine umständliche Anzeige von der Gartenkultur ihres Amts einsenden, und einen ungefähren Überschlag machen, wie viele Obstbäume die dortigen Unterthanen nötig haben. Alsdann werden durch königl. Planteurs die Bäume umsonst gepflanzt. [...] Um einigermaßen die Kosten wieder erstattet zu erhalten, die diese Einrichtung nothwendig mit sich führt, und um wohlhabende Privatpersonen mit guten Obstsorten zu versehen, so werden nunmehr jährlich 2000 Stück hochstämmige, und eben so

\footnotetext{
8 Palm H (2007) Königliche Gartenbibliothek Herrenhausen. Erarbeitung eines Forschungsprogramms. In: Leibniz Universität Hannover. Zentrum für Gartenkunst und Landschaftsarchitektur. Bericht 2005-2007. Hannover. S. 30f. Hier S. 30.

9 Preißel U, Preißel HG (2006) Pflanzen im Berggarten. In: M. v. König (ed) Herrenhausen. Wallstein, Göttingen. S. 195ff. Hier: S. 195.

${ }^{10}$ Kirsch R (1993) Frühe Landschaftsgärten im niedersächsischen Raum. Cuvillier, Göttingen.

11 Boeck UH (2000) Gartenkunst in Niedersachsen vom Mittelalter bis zum Ende des 18. Jahrhunderts. In: Heimatbund Niedersachsen e. V., Niedersächsische Gesellschaft zur Erhaltung historischer Gärten e. V. (ed) Historische Gärten in Niedersachsen, Katalog zur Landesausstellung. Hannover. S. 26f.
} 
viele niedrigstämmige Obstbäume, für einen in den öffentlichen Blättern bestimmten

Preis verkauft." 12

Die Baumschule bot neben Obstbäumen noch andere Gehölze an, vorwiegend aus Nordamerika sowie seltene Gewächse aus dem asiatischen Raum. Hirschfeld wies darauf hin und veröffentlichte auch einen Auszug aus dem Verzeichnis der zum Verkauf stehenden Gehölze. Er lobte deren Qualität und empfahl ausdrücklich die seiner Meinung nach noch wenig bekannten Arten zum Kauf. ${ }^{13}$ Die Arbeiten Hirschfelds sind für die Geschichte der Gartenkunst in der zweiten Hälfte des 18. Jahrhunderts in Deutschland von herausragender Bedeutung und daher bereits mehrfach Gegenstand verschiedener Forschungsprojekte gewesen ${ }^{14}$. In jüngsten Untersuchungen über die Herrenhäuser Gärten ist auch die Geschichte der Plantage dargestellt und auf die Bedeutung dieser Einrichtung aufmerksam gemacht. ${ }^{15}$

\section{Die Ämter und ihre Gärten}

Im Gebiet des heutigen Bundeslandes Niedersachsen bestand bis in die erste Hälfte des 19. Jahrhunderts ein zweischichtiger Behördenaufbau mit einer Zentral- und verschiedenen Ortsinstanzen, die auf einen gut überschaubaren regionalen Bereich zugeschnitten waren. Während die Zentralinstanz (Kammer, Steuerkollegium, Konsitorien u. a.) sich trotz der Personalunion mit England stetig festigte und auf den sich im 17. Jahrhundert gegen Celle, Braunschweig und Wolfenbüttel durchsetzenden hannoverschen Hof konzentrierte, bestanden die örtlichen Verwaltungsinstanzen aus einer Vielzahl sehr verschiedener Einheiten, an deren Spitze in der Regel Angehörige der adligen Familien standen. Neben den Rittergütern, den Patrimonialgerichten und anderen sind es vor allem die Ämter, die bereits im 16. Jahrhundert meist aus mittelalterlichen Burgstellen entstanden waren und als Vorläufer der heutigen Landkreise angesehen werden können. Um 1800 gab es auf niedersächsischem Gebiet über 150 Ämter. ${ }^{16}$ Sie bildeten ein dichtes Netz, das über die rein administrativen Aufgaben hinaus wichtige Impulse zur kulturellen, politischen und gesellschaftlichen Entwicklung des Landes gab.

\footnotetext{
${ }^{12}$ Hirschfeld CCL (1782) Gartenkalender auf das Jahr 1782. Hirschfeld, Kiel, Dessau. S. $129 f f$.

13 Ebd., S. 244ff.

14 Kehn W (1992) Christian Cay Lorenz Hirschfeld 1742-1792. Eine Biographie. Werner, Worms; Schepers W (1980) Hirschfelds Theorie der Gartenkunst 1779-1785. Werner, Worms.

15 Palm H, Rettich H (2006) Die Geschichte des Berggartens. In: M. v. König (ed) Herrenhausen. Wallstein, Göttingen. S. 165.

16 Der „Atlas des Fürstentums Lüneburg“, den Johannes Mellinger im Jahr 1600 unter dem Titel „Lunaeburgici Ducatus“ veröffentlichte, gibt allein für das Gebiet, das etwa ein Fünftel des heutigen Niedersachsen umfasst, 42 Ämter an. Im 18. Jh. sind weitere Ämter gebildet worden, so dass ihre Zahl noch höher liege dürfte. Die heutigen 38 Landkreise sind in der Regel aus fünf oder sechs Ämtern gebildet worden. Hinzu kommen die Ämter der heutigen kreisfreien Städte.
} 


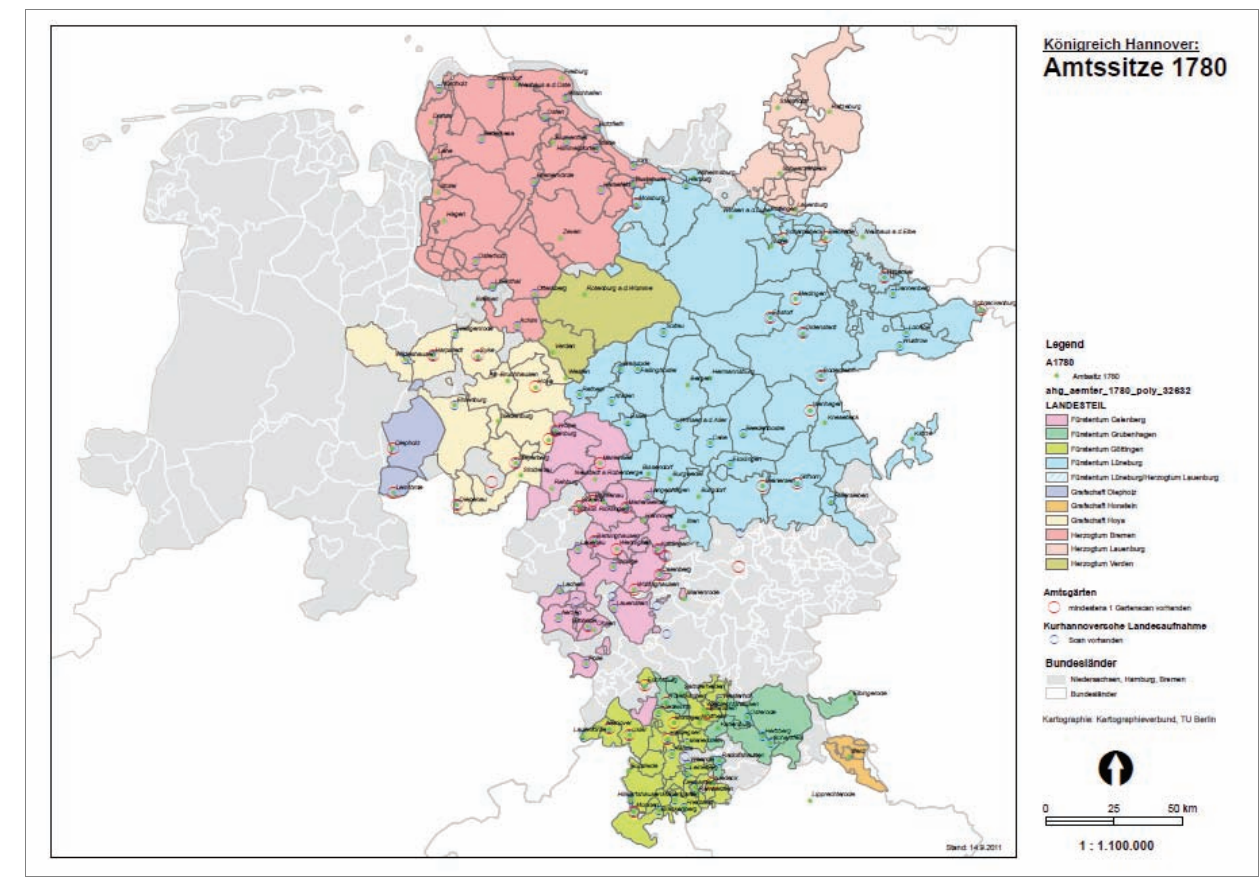

Abb. 1: Übersichtskarte mit der Verteilung der Amtssitze im Königreich Hannover um 1840 (Quelle: TU Berlin, Kartografieverbund).

Durch Beschlüsse des Wiener Kongresses wurde das Kurfürstentum Braunschweig-Lüneburg mit anderen Territorien vereinigt und zum Königreich Hannover erhoben. Das deutlich vergrößerte und von den napoleonischen Reformen in seinem Staatsverständnis völlig veränderte welfische Einflussgebiet musste auch im Hinblick auf seine Funktionsfähigkeit neu organisiert werden. König Georg IV. erließ daher 1822 das „Edikt, die Bildung der künftigen Staatsverwaltung in dem Königreich Hannover betreffend“, das die Schaffung einer Mittelbehörde zum Inhalt hatte. Es wurden die sechs Landdrosteien Hannover, Hildesheim, Lüneburg, Stade, Osnabrück und Aurich eingerichtet, die Vorläufer der erst kürzlich aufgelösten Bezirksregierungen. Damit war ein modernes Verwaltungssystem geschaffen worden, das zwar noch immer von den adligen Familien beherrscht wurde, die beispielsweise die Landdroste stellten. Aber gleichzeitig wurden die unteren Instanzen geschwächt und ihrer teilweise noch aus dem Mittelalter stammenden Privilegien enthoben. In der Folge setzte ein sich durch das gesamte 19. Jahrhundert ziehender Prozess ein, der durch andere Entwicklungen („Bauernbefreiung“ u. a.) noch beschleunigt wurde und die Rechte und Einflussmöglichkeiten der adligen Familien stark einschränkte. ${ }^{17}$ Auch die Ämter wurden nun zunehmend mit Fach-

${ }^{17}$ Hindersmann U (2001) Der ritterschaftliche Adel im Königreich Hannover. Hahn, Hannover. 
leuten aus der Verwaltung besetzt und nicht mehr automatisch an Angehörige der adeligen Familien vergeben.

Damit verbunden war ein insgesamt nachlassender Einfluss der Ämter. Waren sie noch im 18. Jahrhundert regionale Zentren des Kulturtransfers im umfassenden Sinne mit häufig repräsentativer Funktion (Abb. 2), so wandelten sie sich im Zuge der Verwaltungsreform zu reinen Aufsichtsbehörden, von denen keine politischen, gesellschaftlichen und kulturellen Impulse mehr ausgingen. Im 18. Jahrhundert hingegen waren die Ämter Vorposten neuer Strömungen, was sich heute noch an den erhaltenen Gebäuden ablesen lässt.



Abb. 2: Entwurf für einen Neu- bzw. Umbau des Amtshauses in Rotenburg von 1744. Das Amtshaus sollte im Sinne einer modernen Verwaltung funktional und schlicht, aber dennoch repräsentativ und zeitgemäß erscheinen. Der Entwurf für Rotenburg zeigt dies exemplarisch durch den zweigeschossigen Aufbau, die Symmetrie der Fassade und die Klarheit der Grundrissbildung. Das Gebäude hebt sich aus der einfachen ortstypischen Bebauung deutlich heraus, wirkt aber durch das sichtbare Fachwerk nicht aufwendig oder verschwenderisch (Quelle: Niedersächsisches Landesarchiv, Standort Stade). 
Die Gartenkultur spielte eine Schlüsselrolle in dem Organismus der Ämter. An jedem Amtshaus gab es einen Garten, der über seine repräsentative Funktion hinaus der örtlichen Bevölkerung als Anschauungsobjekt dienen sollte und vor allem eine wichtige Funktion bei der Verbreitung der Gartenkultur erfüllte. Denn die Amtshausgärten waren Teil des von Hirschfeld beschriebenen kulturellen und entwicklungspolitischen Systems, mit dem von Hannover ausgehend die Förderung der Gartenkultur vorangetrieben wurde. Die im ganzen Land verteilten Gärten dienten dabei im modernen Sinn als Multiplikatoren und Verknüpfungspunkte eines weiten Netzwerks. Sie bildeten Orte der Anschauung, an denen die in Hannover und anderen gartenkulturellen Zentren gewonnenen oder fortentwickelten Erkenntnisse - teilweise modifiziert und den örtlichen Gegebenheiten angepasst exemplarisch gezeigt und an die Bevölkerung weitergegeben wurden.

Einige Ämter verfügten über sehr große Gärten (Abb. 3), die u. a. auch als örtliche Baumschulen fungierten und eine wichtige Rolle bei der Verbreitung von Gehölzen spielten. ${ }^{18}$ Andere waren durch ihre Gestaltung bedeutsam (Abb. 4) und brachten der örtlichen Bevölkerung die neuesten Entwicklungen der Gartenkunst nahe. ${ }^{19}$ In vielen Fällen wurde das den Ämtern von der Hannoverschen Plantage angebotene Schulungsprogramm zur Aus- bzw. Weiterbildung von Gärtnern in Anspruch genommen, so dass auch das Wissen über neueste Techniken und Methoden der Obst- und Ziergärtnerei von den Ämtern aus weitergegeben wurde.

\footnotetext{
${ }^{18}$ Für den Obstgarten des Amts Westen südlich von Verden sind für das frühe 18. Jahrhundert über 1200 Bäume dokumentiert (Inventar des Amts Westen von 1756, Kreisarchiv Verden Nr. 10g/4). In den Gutsgärten sind in der Regel 150-300 Bäume dokumentiert, vgl. dazu Beck (2009).

${ }_{19}$ Ein Beispiel für einen bedeutenden barocken Amtshausgarten ist Stotel bei Bremerhaven („Plan von dem Amthofe und Garten zu Stotel“", Staatsarchiv Stade, Karte 43 d Stotel Nr.7). Ein anderer bedeutender Garten war an dem zum Amtshaus umgebauten sog. Schloss in Rotenburg/Wümme vorhanden (siehe dazu: Burg und Schloß in Rotenburg, Archäologische und physikalische Untersuchungen 1986-1994, o. A. In: Archäologische Berichte des Landkreises Rotenburg (Wümme), Nr. 6/1996). U. a. ein Lageplan (,Situations Plan vom Amtshofe in Rotenburg“ von 1835, Staatsarchiv Stade, Karte 421 Rotenburg Nr. 11) dokumentiert diesen Garten.
} 


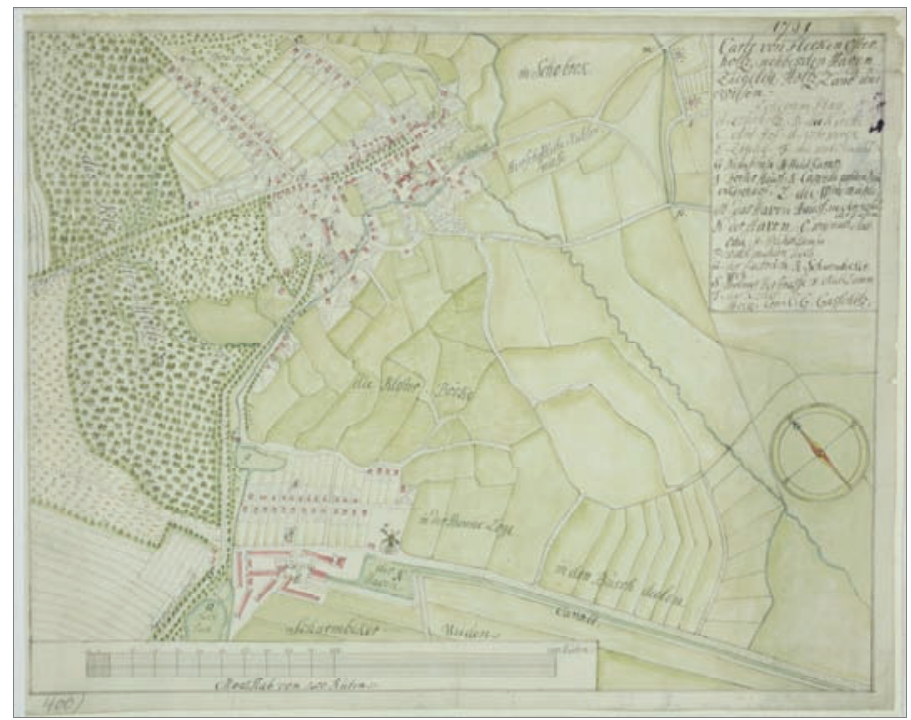

Abb 3: Übersichtsplan von Osterholz, 1731. Der Amtsbezirk bildet einen großen Komplex, zu dem verschiedene, große Gärten gehören (Quelle: Niedersächsisches Landesarchiv, Standort Stade).

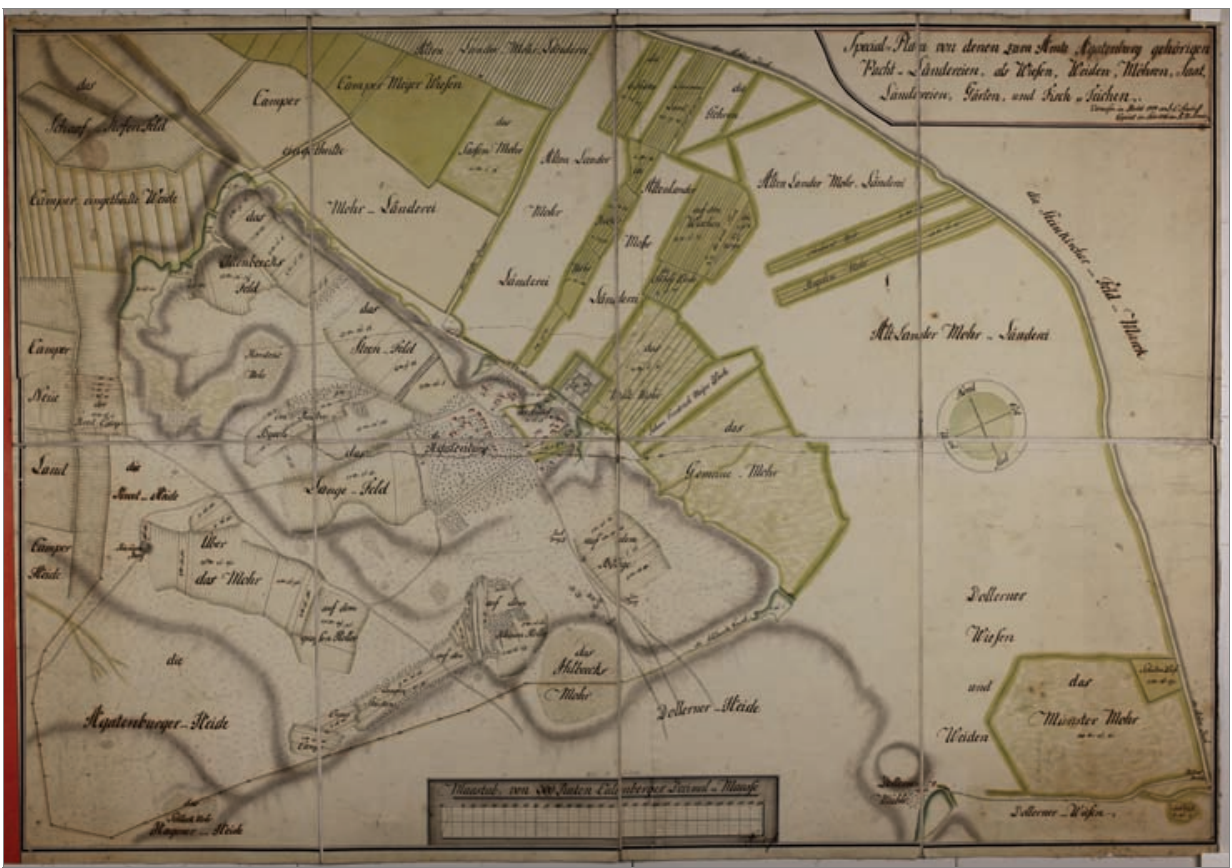

Abb. 4: Plan des Amtssitzes Agathenburg, 1774/1776. Der große Garten zeigt eine künstlerisch sehr aufwendige, für den ländlichen Raum ungewöhnliche Formsprache (Quelle: Niedersächsisches Landesarchiv, Standort Stade). 
Obwohl die Gärten der Amtshäuser mit fast 200 Anlagen (erste Hälfte 19. Jahrhundert) eine sehr große Gruppe unter den ländlichen Gärten Niedersachsens bildeten, sind bisher weder ihre Geschichte noch die künstlerische Gestaltung und Bedeutung in Forschungsarbeiten thematisiert worden. ${ }^{20}$ Gleiches gilt für die Amtshäuser, deren Bedeutung als gesellschaftspolitische Orte und kulturelle Zentren im ländlichen Raum noch nicht erkannt worden ist. ${ }^{21}$

Die bisherigen Arbeiten zur Geschichte der Verwaltung beschränken sich meist auf Fragen der Organisation, der Zuständigkeit und dem Verhältnis der einzelnen Verwaltungsebenen und -einheiten zueinander. Seltener wird die gesellschaftspolitische Rolle der Verwaltung thematisiert oder die kulturellen Einflüsse der sich vor allem im 19. Jahrhundert in großen Prachtbauten manifestierenden Administration. ${ }^{22}$ Dabei zeigt gerade das Wirken einzelner Persönlichkeiten, dass mit dem Aufbau der flächendeckenden Verwaltung im 17. und 18. Jahrhundert nicht nur die Absicht der Regelung und Reglementierung, sondern ausdrücklich auch die Intention der kulturellen Entwicklung des Kurfürstentums und späteren Königreichs verbunden war. ${ }^{23}$ Die Ämter übernahmen in dieser Zeit nicht nur rein administrative Aufgaben, sondern spielten eine wichtige Rolle bei der kulturellen, politischen und gesellschaftlichen Entwicklung des Landes (Abb. 5-7). Im 18. Jahrhundert stellten sie regionale Zentren des Kulturtransfers im umfassenden Sinne dar. Sie waren häufig kulturelle Kristallisationspunkte und Vorposten neuer Strömungen ${ }^{24}$, die Gebäude oft hervorragende Zeugnisse der Baukunst in einem von ländlicher Architektur geprägten Umfeld. ${ }^{25}$ Sehr ehrgeizige Amtmänner statteten ihre Häuser sogar mit erstklassigen Interieurs aus, wie beispielsweise die berühmten „Chinesischen Tapeten“ im Amtshof Eicklingen zeigen. ${ }^{26}$

\footnotetext{
${ }^{20}$ Einzige Ausnahme sind wenige herausragende Objekte, die aber nur zeitweise als Ämter dienten, wie der Garten des Schlosses Agathenburg; vgl. Neumann G (1986) Zur Geschichte des Schlosses Agathenburg und seiner Besitzer. Stade; Wörner R, Wörner G (1991) Denkmalpflegerisches Gutachten zur Wiederherstellung der Gartenanlagen der Agathenburg. Unveröff. Gutachten.

21 „Edikt, die Bildung der künftigen Staatsverwaltung in dem Königreich Hannover betreffend“, zitiert aus: Jeserich KGA, Pohl H, Unruh GC (ed) (1982) Deutsche Verwaltungsgeschichte. DVA, Stuttgart. Vgl. auch Hindersmann (2001).

22 Nolte J (1991) Staatserfordernis und Kunstaufwendung. Hildesheim.

${ }^{23}$ Merker O (1976) Karl August Freiherr von Herdenbergs Reformdenken in seiner hannoverschen Zeit 1771-1781. In: C. Haase (ed) Niedersächsisches Jahrbuch. Bd. 48. August Lax, Hildesheim. S. 325-344.

${ }^{24}$ Vgl. Brüning K, Schmidt H (1986) Handbuch der historischen Stätten Deutschlands. Bd. 2. Niedersachsen und Bremen. Kröner, Stuttgart zum Amthaus Koldingen und Witt G (1981) Amt und Festung Uslar. Solling-Verein, Uslar zum Amtshaus in Uslar.

25 Z.B. das 1727-34 errichtete Amtshaus in Winsen/Aller, ein barocker Massivbau in der sonst von ländliche Fachwerkbauten geprägten Region (Segers-Glocke C (ed) (1994) Denkmaltopographie Bundesrepublik Deutschland. Baudenkmale in Niedersachsen. Landkreis Celle. Niemeyer, Hameln. S. 210f.) oder die Amtshäuser in Burgdorf (Dehio G (1992) Handbuch der Deutschen Kunstdenkmäler, Bremen und Niedersachsen. Dt. Kunstverl., München. S. 324) und in Hardegsen (Segers-Glocke C (Hg.) (2002) Denkmaltopographie Bundesrepublik Deutschland. Baudenkmale in Niedersachsen. Landkreis Northeim. Teil 1. Niemeyer, Hameln. S. 103)

${ }^{26}$ Die Wandtapeten befinden sich heute im Tapetenmuseum in Kassel; Mick EW (1983) Deutsches Tapetenmuseum Kassel. Hessische Brandversicherungsanst. Kassel. S. 141.
} 


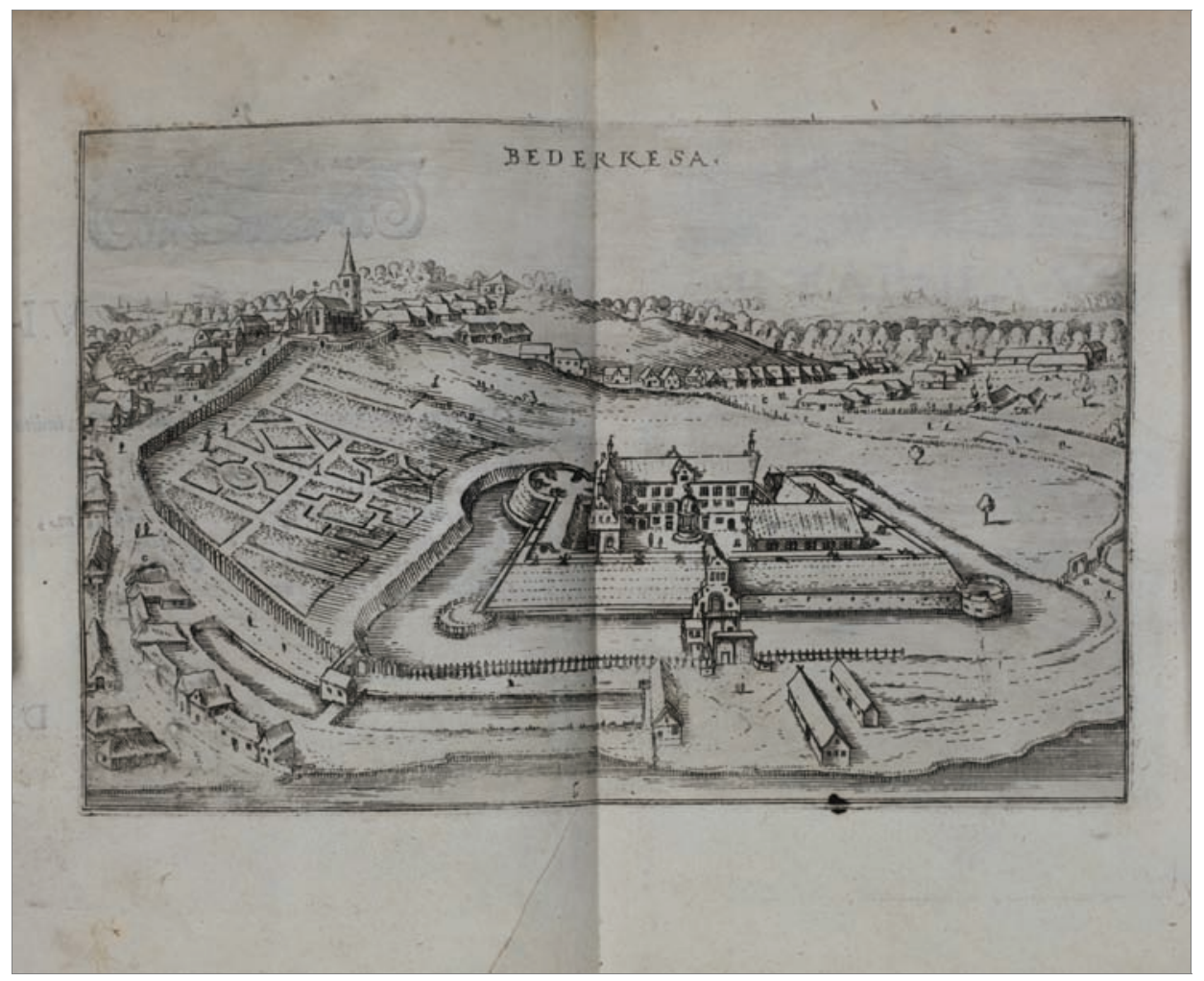

Abb. 5: Ansicht der alten Burg Bederkesa, Stich von Wilhelm Dillich. Die alte Festung der Bremer Bischöfe wurde nach 1720 zum Amt umgebaut, sie ist ein gutes Beispiel für die Erhaltung spätmittelalterlicher, geschichtlich bedeutender Gebäude durch die Folgenutzung als Amtshaus (Quelle: Niedersächsisches Landesarchiv, Standort Stade). 


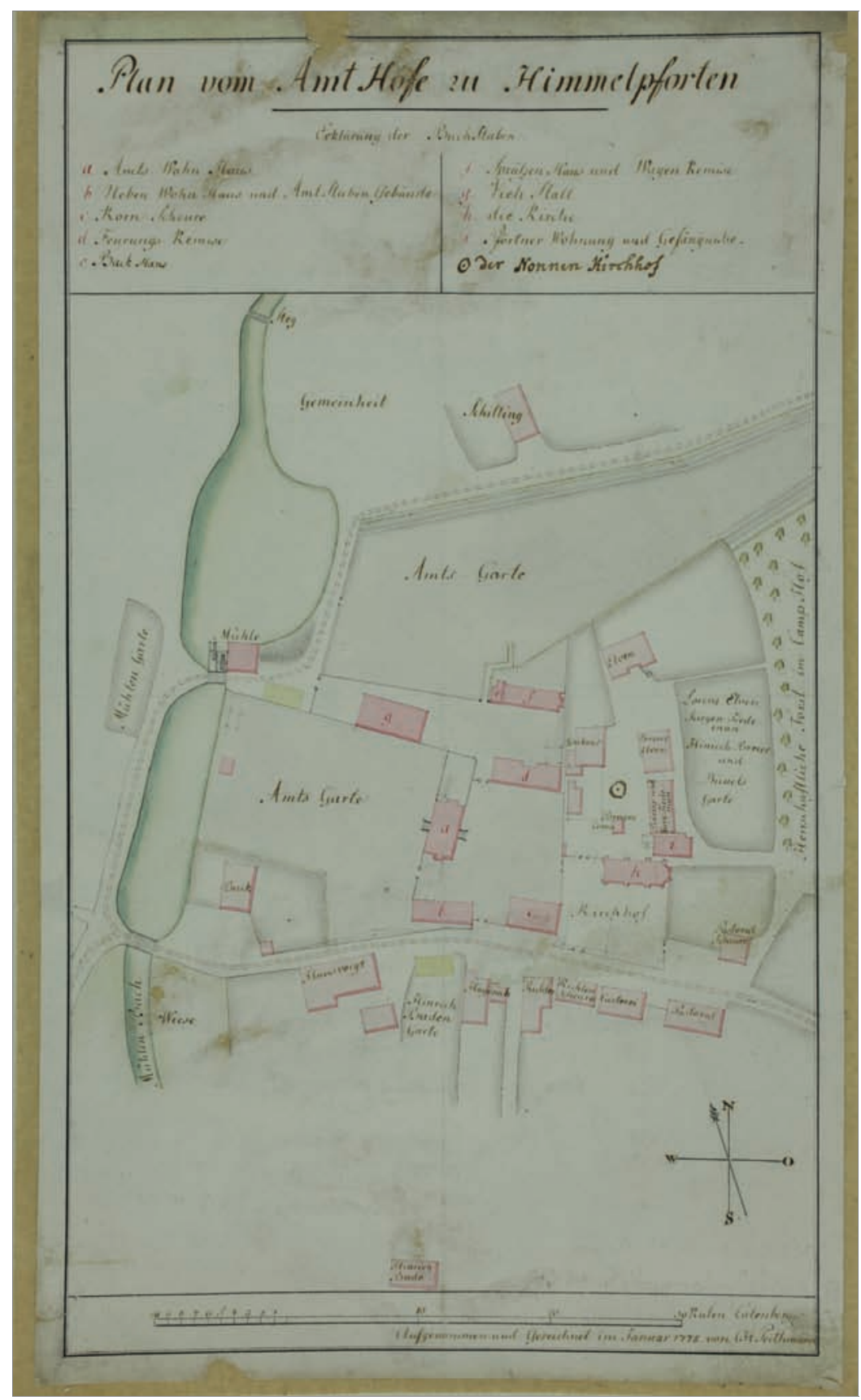

Abb. 6: Plan des Amtssitzes Himmelpforten von 1778. Auch einige Klöster wurden zu Amtssitzen umgebaut und entgingen dadurch ihrer vollständigen Zerstörung. Allerdings blieben oft nur Teile der mittelalterlichen Bausubstanz erhalten, in Himmelpforten beispielsweise der Chorraum der früheren Klosterkirche (Quelle: Niedersächsisches Landesarchiv, Standort Stade). 




Abb. 7: Plan des Amtshauses Ottersberg, 1731. Die alte Burg ist samt ihrer Verteidigungsanlagen immer wieder erneuert worden und blieb durch die Nutzung als Amtshof im 19. Jahrhundert bis heute weitgehend erhalten[Quelle: Niedersächsisches Landesarchiv, Standort Stade).

Die Gärten der Ämter sind durch zahlreiche Pläne dokumentiert. Auch wenn nur sehr wenig über die tatsächliche Ausstattung der Anlagen bekannt ist, lassen sich immerhin die Grundrisse und in einigen Fällen auch einzelne Elemente wie Gartenhäuschen, Alleepflanzungen und Brunnen erkennen (Abb: 8). Die Pläne ermöglichen so auch eine kunsthistorische Einordnung der Gärten. Eine genaue Plananalyse könnte darüber hinaus weitere Erkenntnisse über die Ausprägung der Gartenkunst, die sich in den großen stilbildenden Anlagen entwickelt hat, im regionalen Kontext erbringen. Dazu wäre allerdings eine eigene Untersuchung notwendig. Bereits an Einzelbeispielen ist ansatzweise beschrieben worden, wie die großen Entwicklungslinien der Gartenkunst sich in den Anlagen der ländlichen Regionen in teilweise sehr originellen Variationen wieder finden lassen. ${ }^{27}$ Sie zeigen eine faszinierende Vielfalt und Kreativität beim Umgang mit vermeintlich kanonischen Gestaltungsprinzipien.

\footnotetext{
${ }^{27}$ Dies gilt insbesondere für Objekte, die von großer kultureller und geschichtlicher Bedeutung sind, aber nur zeitweise der Amtsverwaltung dienten. Dazu gehören beispielsweise die frühere Burg Bremervörde (Bachmann E, Brandt R (1987) Bremervörde, Bilder aus der Geschichte einer Stadt. Borgardt, Bremervörde.) oder der sog. Erbhof in Thedinghausen (Gravens F (2001) Der Erbhof Thedinghausen. Thedinghausen).
} 




Abb. 8: Plan des Amtsgartens Lilienthal von 1737. Der Garten weist eine für die Zeit typische Gliederung durch Alleepflanzungen auf, vermutlich aus Obstbäumen (Quelle: Niedersächsisches Landesarchiv, Standort Stade).

\section{Die Verteilung der Obstgehölze über die Ämter}

Die von Hirschfeld beschriebene kostenlose Verteilung von Obstgehölzen bildete das Herzstück des Förderprogramms. In einem Zeitraum von etwa 60 Jahren (ca. 1770-1830) konnte jedes Amt jährlich eine Liste nach Herrenhausen schicken, in der die Personen namentlich aufgeführt waren, die Obstbäume erhalten sollten. Auch die gewünschten Gehölze wurden aufgelistet. Sehr viele dieser Listen sind in den Akten erhalten, ebenso sind die Lieferbücher aus Herrenhausen noch vorhanden, so dass der Prozess gut dokumentiert ist. Die Bäume wurden in Herrenhausen 
auf Karren gepackt, zu den Ämtern gebracht und dort verteilt. In die entferntesten Ämter in Ostfriesland dauerte der Transport fünf Tage, nicht immer kamen die Bäume unversehrt an. Dokumentiert sind auch Klagen über vertrocknete Bäume oder solche, die zu spät geliefert wurden und bereits austrieben. Insgesamt hat aber die Verteilung über Jahre hin gut funktioniert, so dass das Programm insgesamt als erfolgreich bezeichnet werden kann. Dabei sind die Zahlen beeindruckend: Jährlich wurden aus der Plantage 3.000-4.000 Bäume verteilt, etwa 500 pro Drostei. Die Belieferten konnten aus einem umfangreichen Sortiment auswählen, in der Blütezeit der Plantage um 1780 konnten allein über 800 Apfelsorten angeboten werden. Je nach Region, Klima- und Bodenverhältnissen wurden von den Gärtnern in Herrenhausen die Lieferungen zusammengestellt. Besonders rege Personen wurden auch mehrfach bedacht, sie erhielten über mehrere Jahre 6-10 Bäume pro Lieferung und konnten von diesen Mutterbäumen selbst Stecklinge heran ziehen. Auffällig ist, dass einige Pastoren überdurchschnittlich viele Bäume erhielten, in einigen Fällen über 100 Exemplare. Sie legten oft eigene Baumschulen an, um weitere Jungbäume heranziehen und in ihrer Region verkaufen zu können.

Auch die Amtmänner selbst konnten Gehölze aus Herrenhausen beziehen, allerdings nicht kostenlos. Viele unterhielten ebenfalls eigene bzw. amtseigene Baumschulen, die zur weiteren Verbreitung der Bäume beitrugen. Auch Privatpersonen wurden angehalten, Baumschulen anzulegen; dies sollte wiederum der Verbreitung von Gehölzen dienen und gleichzeitig neue Erwerbsmöglichkeiten erschließen.

Nach 1830 wurde zunehmend Kritik an dem Verteilungssystem geübt. Einerseits werden die hohen Kosten der Herrenhäuser Plantage angeführt, andererseits die Konkurrenz zu den neu entstandenen privaten Baumschulen betont, die deren Existenz gefährde und damit gerade den erhofften Effekt - die Gründung privater Baumschulen im ganzen Land - gefährde. Daher wurde nach und nach der Versand aus Herrenhausen eingestellt, die Ämter erhielten nun direkte Geldzahlungen, von denen sie bei örtlichen Baumschulen Gehölze kaufen und verteilen konnten. Damit verlor die Plantage ihre ursprüngliche Zweckbestimmung und wurde folgerichtig gegen Ende des Jahrhunderts aufgegeben. Die Verteilung von Obstbäumen an die Bevölkerung wurde jedoch von einigen Landkreisen bis in die Nachkriegszeit fortgeführt.

\section{Literatur zum Thema Obstbau}

Die Bemühungen zur Förderung der Gartenkultur und insbesondere des Obstbaus beschränkten sich nicht auf die kostenlose Verteilung von Gehölzen. Es wurden auch verschiedene Schriften, Anleitungen und Handbücher zu Themen der Baumzucht, der Lagerung und Verarbeitung des Obstes, der Anlage von Baumschulen u. ä. gefördert und teilweise wiederum kostenlos der Bevölkerung zur Verfügung gestellt. Auch dies erfolgte über die Ämter. Einige verfügten sogar über einen ge- 
wissen Bestand an Büchern zu dem Thema, die von interessierten Personen ausgeliehen werden konnten. Auch in der Hausväterliteratur wird der Obstbau umfassend abgehandelt.

In den 1760er und 1770er Jahren entstehen einige wichtigste Standardwerke; 1761 erschien die kurze Abhandlung „Vom Nutzen wilder Baumschulen“ von Rammelt ${ }^{28}$, 1763 die erste deutsche Übersetzung des viel gelesenen französischen Werks von Duhamel „Von den Baumschulen, von der Holzsaat, und Pflanzung der Waldbäume" ${ }^{29}$. Im Forsthandbuch von Guiot 1771 ist die Anlage von Forstbaumschulen ausführlich beschrieben, bezüglich der Anzucht von Obstgehölzen verfasst 1769 der Pastor Samuel David Ludwig Henne († 1782) ein wichtiges, mehrfach wieder aufgelegtes und weit verbreitetes Werk. Seine „Anweisung wie man eine Baumschule von Obstbäumen im Großen anlegen und gehörig unterhalten solle“, die 1772, 1774 und 1791 in zweiter bis vierter Auflage erscheint, beruht auf eigenen Erfahrungen, die Henne in seiner Mitte der 1750er Jahre angelegten Obstbaumschule gewonnen hat. ${ }^{30}$ Etwa zeitgleich schreibt Johann Gottlieb Jacobi den Aufsatz „Von Anlegung und Wartung guter Baumschulen“, der von Otto von Münchhausen in seinem Werk „Der Hausvater“ im 5. Teil 1770 veröffentlicht wird $^{31}$. Christian Cay Lorenz Hirschfeld ${ }^{32}$ empfiehlt die ab 1770 in Auszügen und 1782 vollständig erschienene „Anleitung zur Anlegung, Wartung und Erhaltung eines Obstgartens aus eigenen Bemerkungen“ von Johann Heinrich Pratje seinen Lesern. Das Buch war vor allem im norddeutschen Raum verbreitet und enthält ein umfangreiches Kapitel über die Einrichtung einer Baumschule. ${ }^{33}$

Neben den Handbüchern werden in dieser Zeit mehrere kürzere Artikel zum Thema Obstbaumschulen veröffentlicht, z. B. im Hannoverschen Magazin 1767 und $1770^{34}$, im Leipziger Intelligenzblatt $1768^{35}$ oder im Stuttgarter allgemeinen

\footnotetext{
${ }^{28}$ Rammelt G (1761) Abhandlung vom Nutzen wilder Baumschulen. In: D. G. Schrebers (ed) Sammlung verschiedener Schriften etc., 8. Teil. Curts, Halle. S. 383-387. Angeführt von Krünitz (1774) Oeconomischen Encyclopädie, oder allgemeines System der Staats-, Stadt-, Haus- und Landwirtschaft, in alphabetischer Ordnung. 4. Teil. Berlin. S. 92.

${ }_{29}$ Duhamel du Monceau, HL (1763) Von der Holz-Saat und Pflanzung der Wald-Bäume. Auch derselben ferneren Wart [...] Durchaus m. vielen Kupferstichen. Durch Du Hamel Du Monceau. Aus d. Franz. übers. durch Carl Christoph Oelhafen. Seligmann, Nürnberg.

${ }^{30}$ Henne SDL (1791) Anweisung, wie man eine Baumschule von Obstbäumen im Großen anlegen und gehörig unterhalten solle. Wobey eine vollständige Beschreibung der vornehmsten darinn vorkommenden Obstsorten befindlich, nebst einer deutlichen Anweisung zum Pfropfen und Okulieren, und einer ganz neuen Abhandlung vom Kopulieren. JC Hendel, Halle. S. 39.

${ }^{31}$ Jacobi JG (1770) Von Anlegung und Wartung guter Baumschulen. In: O. v. Münchhausen (ed) Der Hausvater. 5. Teil. 2. Stück. Förster, Hannover. S. 507-542.

32 Hirschfeld (1782), S. 37.

33 Pratje JH (1782) Anleitung zur Anlegung, Wartung und Erhaltung eines Obstgartens aus eigenen Bemerkungen, in Briefen. Dietrich, Göttingen.

${ }^{34} \mathrm{~K}$. Anmerkungen über die einländische Erzielung guter Garten- und Futtersämerey, und die Auferziehung tüchtiger Obstbäume. Im 85. St. des Hannoverschen Magazins 1767; Briefe über die Anlegung, Wartung und Erhaltung eines Obst-Gartens. Im 18., 41. und 43. St. des Hannoverschen Magazins 1770. Angeführt von Krünitz (1774), S. 92.

35 Rammelt (1768), S. 27-29.
} 
Magazin 176736. Auf der Basis dieses Wissens entsteht 1774 im 4. Teil der Oeconomischen Encyclopädie von Johann Georg Krünitz (1728-1796) der 48 Seiten umfassende Eintrag zum Stichwort „Baum-Schule“.

Gegen Ende des 18. Jahrhunderts ist eine erneute Zunahme der Literatur zum Thema Obstbau und Obstbaumschulen zu beobachten. 1791 und 1792 verfasste Johann Jacob Meyen in Stettin zwei Bände über Obstbaumzucht und die ,wilde Baumzucht“, die er 1793 zusammen als „Physikalisch-ökonomische Baumschule“ herausgab. Dieses Buch wendete sich als Empfehlung an die Landwirte ${ }^{37}$, die angeregt werden sollen, Baumschulen anzulegen und Obstgärten anzulegen, um mit den Früchten Handel zu treiben. Dies würde den Wohlstand in der gesamten Region fördern, denn ein vermehrter Geldumsatz käme auch dem Handwerk und dem Luxusgüterhandel zugute. In Frankfurt erschien 1797 von Johann Ludwig Christ (1739-1813) das „Handbuch über die Obstbaumzucht und Obstlehre“, das mehrere Auflagen erlebte und in ganz Deutschland gelesen wurde. In der 3. Auflage von 1804 kann der Autor bereits Erfolge der allgemeinen Förderung von Baumschulen vermelden:

„Fast in allen Gegenden entstehen nicht nur kleinere Privatbaumschulen, sondern auch viele große, unter Aufsicht geschickter und einsichtsvoller Männer: und die französischen Baumschulen, - die ohnedem für unser Klima nur kurzdaurende (sic!) Bäume lieferten, - werden entbehrlich“"38.

1806 verweist von Schönebeck in seinem Lehrbuch, das sich auch der „Anlegung einer Baumschule im Großen“" widmet, schon auf eine lange Liste von mehr als 22 Autoren, die sich zu diesem Thema geäußert haben. ${ }^{39}$

Neben den Lehr- und Handbüchern erscheint Ende des 18. Jahrhunderts die erste Fachzeitschrift, die sich speziell dem Obstbau widmet: der von Johann Volkmar Sickler (1742-1820) herausgegebene „Teutsche Obstgärtner“, von 1794 bis 1804 in 22 Bänden publiziert. Ziel dieser Zeitschrift ist die Förderung und Bekanntmachung des Obstbaus. Angesprochen werden sollen nach eigenen Angaben Bürger und Bauern..$^{40}$ In den ersten zehn Jahrgängen gibt es einige Kapitel speziell zur Anlage von Obstbaumschulen.

\footnotetext{
36 Thymmen JW (1767) Kurzer Entwurf zur Einrichtung einer allgemeinen Baum-schule. In: Stuttgarter allgemeines Magazin. Jg. 1767. JC Erhard, Stuttgart. S. 454-460.

37 Meyen JJ (ed) (1793) Physikalisch-ökonomische Baumschule. 2 Teile. Stettin. S. III, Vorrede.

38 Christ JL (1804) Handbuch über die Obstbaumzucht und Obstlehre. Hermann, Frankfurt. S. XV, Vorwort zur 2. Auflage.

39 Schönebeck C v. (1806) Vollständige Anleitung zur Vermehrung und Pflege der Obstbäume und zur Anlegung einer Baumschule im Großen, nebst einer kurzen Uebersicht der Geschichte der Obstcultur. Köln. S. 396.

40 Sickler JV (1794) Baumschul-Wesen. Vom Verpflanzen der jungen Bäume in die Obst-Plantage. In: Der teutsche Obstgärtner. Bd. 4. Verlag des Industrie-Comptiors, Weimar. S. 40-55. Hier S. 3
} 


\section{$8 \quad$ Fazit}

Im Kurfürstentum Hannover ist in der zweiten Hälfte des 18. und der ersten Hälfte des 19. Jahrhunderts ein systematischer, auf die Strukturen der Verwaltung gestützter Kulturtransfer betrieben worden. Die treibende Kraft war König Georg III., dessen gärtnerische, land- und forstwirtschaftliche Interessen den Anstoß dazu gaben. Die von Herrenhausen mit der Plantage als Mittelpunkt ausgehende Verteilung von Obstbäumen über die Ämter war dabei das wichtigste Instrument. Das beschriebene System ist ein wichtiges Beispiel für eine Form der regionalen Landentwicklung und auch der historischen Netzwerkbildung. Den Ämtern kam dabei eine entscheidende Funktion zu. Denn sie standen vermittelnd an der Schnittstelle zwischen der Zentralregierung und der örtlichen Bevölkerung und zeigen in ihrer Ausformung und ihrer Einbindung in eine umfassende Organisationsstruktur exemplarisch die konkrete Umsetzung der gesellschafts- und kulturpolitischen Absichten der damaligen Regierung. Damit bietet das Thema ein positives Beispiel dafür, dass die Verwaltung eines Landes als kreatives Instrument verstanden und genutzt werden kann, um bestimmte entwicklungspolitische Ziele umzusetzen. Verwaltung bedeutet nicht nur die möglichst reibungslose Organisation der staatlichen Gewalt, sondern kann auch innovative Ideen umsetzen, zukunftsfähige Strukturen schaffen, langfristige Ziele verfolgen und nachhaltige, gesellschaftliche Impulse geben. Gleichzeitig scheint gerade in der Ausprägung einer relativ guten Organisation der entscheidende Unterschied zu ähnlichen Programmen in anderen deutschen Staate zu liegen (Landgrafschaft Hessen-Kassel, Kurfürstentum Sachsen und dem preußischen Königreich), in denen zwar bereits früher Verordnungen zur Förderung des Obstbaus erlassen wurden, diese aber mangelnder Erzeuger- und Vertriebsstrukturen auch durch staatlichen Druck nicht wirkungsvoll umgesetzt werden konnten. Der nachhaltige Erfolg des Hannoverschen Vorgehens lässt sich an der Tatsache ablesen, dass in Niedersachsen bis heute der Obstbau und auch das Baumschulwesen eine tragende Rolle spielen und nach wie vor in einigen Regionen schwerpunktmäßig betrieben werden.

\section{Literatur}

Alpers S (1998) Kunst als Beschreibung. Holländische Malerei des 17. Jahrhunderts. Du Mont, Köln.

Bachmann E, Brandt R (1987) Bremervörde, Bilder aus der Geschichte einer Stadt. Borgardt, Bremervörde.

Beck J (2009) Historische Gutsgärten im Elbe-Weser-Raum. Dissertation an der Fakultät Architektur und Landschaft der Leibnizuniversität Hannover. Stade. 
Benjamin W (1974) Über den Begriff der Geschichte. In: R. Tiedemann, H. Schweppenhäuser (ed) W. Benjamin. Gesammelte Schriften. Bd. 2. Teil 2. Suhrkamp, Frankfurt. S. 698-699.

Boeck UH (2000) Gartenkunst in Niedersachsen vom Mittelalter bis zum Ende des 18. Jahrhunderts. In: Heimatbund Niedersachsen e. V., Niedersächsische Gesellschaft zur Erhaltung historischer Gärten e. V. (ed) Historische Gärten in Niedersachsen, Katalog zur Landesausstellung. Hannover. S. 15-30.

Brown B, Aaron M (2001) The politics of nature. In: Smith J (ed) The rise of modern genomics. 3rd ed. Wiley, New York. S. 795-812.

Brüning K, Schmidt H (1986) Handbuch der historischen Stätten Deutschlands. Bd. 2. Niedersachsen und Bremen. Kröner, Stuttgart.

Buttlar A v. (2003) Der historische Garten als Gegenstand der wissenschaftlichen Forschung im 20. Jahrhundert. In: Vereinigung der Landesdenkmalpfleger in der Bundesrepublik Deutschland und Landesamt Berlin (ed) Historische Gärten. Berlin.

Christ JL (1804) Handbuch über die Obstbaumzucht und Obstlehre. Hermann, Frankfurt.

Dehio G (1992) Handbuch der Deutschen Kunstdenkmäler, Bremen und Niedersachsen. Dt. Kunstverl., München.

Duhamel du Monceau, HL (1763) Von der Holz-Saat und Pflanzung der WaldBäume. Auch derselben ferneren Wart [...] Durchaus m. vielen Kupferstichen. Durch Du Hamel Du Monceau. Aus d. Franz. übers. durch Carl Christoph Oelhafen. Seligmann, Nürnberg.

Franz G (1984) Geschichte des deutschen Gartenbaus. Stuttgart.

Gartenbauverein für das Königreich Hannover (1833) Verhandlungen des Gartenbauvereins für das Königreich Hannover. Heft 1. Hannover.

Gravens F (2001) Der Erbhof Thedinghausen. Thedinghausen.

Henne SDL (1791) Anweisung, wie man eine Baumschule von Obstbäumen im Großen anlegen und gehörig unterhalten solle. Wobey eine vollständige Beschreibung der vornehmsten darinn vorkommenden Obstsorten befindlich, nebst einer deutlichen Anweisung zum Pfropfen und Okulieren, und einer ganz neuen Abhandlung vom Kopulieren. JC Hendel, Halle.

Hindersmann U (2001) Der ritterschaftliche Adel im Königreich Hannover. Hahn, Hannover.

Hirschfeld CCL (1782) Gartenkalender auf das Jahr 1782. Hirschfeld, Kiel, Dessau. 
Hülsmann B (1982) 150 Jahre Gartenbauverein der Hauptstadt Hannover 18321982. Hannover.

Jacobi JG (1770) Von Anlegung und Wartung guter Baumschulen. In: O. v. Münchhausen (ed) Der Hausvater. 5. Teil. 2. Stück. Förster, Hannover. S. 507-542.

Jeserich KGA, Pohl H, Unruh GC (ed) (1982) Deutsche Verwaltungsgeschichte. DVA, Stuttgart.

Kehn W (1992) Christian Cay Lorenz Hirschfeld 1742-1792. Eine Biographie. Werner, Worms.

Kirsch R (1993) Frühe Landschaftsgärten im niedersächsischen Raum. Cuvillier, Göttingen.

König M v. (2006) Herrenhausen. Die königlichen Gärten in Hannover. Wallstein, Göttingen.

Krünitz JG (1774) Oeconomischen Encyclopädie, oder allgemeines System der Staats-, Stadt-, Haus- und Landwirtschaft, in alphabetischer Ordnung. 4. Teil. Berlin.

Marshall TG, Marshall FE (2003) New treatments emerge as sarcoidosis yields up its secrets. ClinMed NetPrints.

http://clinmed.netprints.org/cgi/content/full/2003010001v1, zuletzt besucht am 24. Juni 2004

Mellinger J (2001) Atlas des Fürstentums Lüneburg um 1600. Verl. Für Regionalgeschichte, Bielefeld.

Merker O (1976) Karl August Freiherr von Herdenbergs Reformdenken in seiner hannoverschen Zeit 1771-1781. In: C. Haase (ed) Niedersächsisches Jahrbuch. Bd. 48. August Lax, Hildesheim. S. 325-344.

Meyen JJ (ed) (1793) Physikalisch-ökonomische Baumschule. 2 Teile. Stettin.

Mick EW (1983) Deutsches Tapetenmuseum Kassel. Hessische Brandversicherungsanst., Kassel.

Neumann G (1986) Zur Geschichte des Schlosses Agathenburg und seiner Besitzer. Stade.

Nolte J (1991) Staatserfordernis und Kunstaufwendung. Hildesheim.

Palm H (2007) Königliche Gartenbibliothek Herrenhausen. Erarbeitung eines Forschungsprogramms. In: Leibniz Universität Hannover. Zentrum für Gartenkunst und Landschaftsarchitektur. Bericht 2005-2007. Hannover. S. 30-35. 
Palm H, Rettich H (2006) Die Geschichte des Berggartens. In: M. v. König (ed) Herrenhausen. Wallstein, Göttingen. S. 165-149.

Pratje JH (1782) Anleitung zur Anlegung, Wartung und Erhaltung eines Obstgartens aus eigenen Bemerkungen, in Briefen. Dietrich, Göttingen.

Preißel U, Preißel HG (2006) Pflanzen im Berggarten. In: M. v. König (ed) Herrenhausen. Wallstein, Göttingen. S. 195-206.

Rammelt G (1761) Abhandlung vom Nutzen wilder Baumschulen. In: D. G. Schrebers (ed) Sammlung verschiedener Schriften etc., 8. Teil. Curts, Halle. S. 383-387.

Rammelt G (1768) Abhandlungen von Baumschulen. In: Leipziger Intelligenzblatt, Nr. 3. Leipzig.

Sablowski M (2006) Zur Geschichte des Gartenbauvereins der Hauptstadt Hannover e. V. 1832-2005. Hannover.

Schepers W (1980) Hirschfelds Theorie der Gartenkunst 1779-1785. Werner, Worms.

Schönebeck C v. (1806) Vollständige Anleitung zur Vermehrung und Pflege der Obstbäume und zur Anlegung einer Baumschule im Großen, nebst einer kurzen Uebersicht der Geschichte der Obstcultur. Köln.

Segers-Glocke C (ed) (1994) Denkmaltopographie Bundesrepublik Deutschland. Baudenkmale in Niedersachsen. Landkreis Celle. Niemeyer, Hameln.

Segers-Glocke C (Hg.) (2002) Denkmaltopographie Bundesrepublik Deutschland. Baudenkmale in Niedersachsen. Landkreis Northeim. Teil 1. Niemeyer, Hameln.

Sickler JV (1794) Baumschul-Wesen. Vom Verpflanzen der jungen Bäume in die Obst-Plantage. In: Der teutsche Obstgärtner. Bd. 4. Verlag des IndustrieComptiors, Weimar. S. 40-55.

Thymmen JW (1767) Kurzer Entwurf zur Einrichtung einer allgemeinen Baumschule. In: Stuttgarter allgemeines Magazin. Jg. 1767. JC Erhard, Stuttgart.

Witt G (1981) Amt und Festung Uslar. Solling-Verein, Uslar.

Wörner R, Wörner G (1991) Denkmalpflegerisches Gutachten zur Wiederherstellung der Gartenanlagen der Agathenburg. unveröff. Gutachten.

South J, Blass B (2001) The future of modern genomics. Blackwell, London. 


\title{
Nutz, Pflicht und Vergnügen: \\ Umweltwahrnehmungen im europäischen Landwirtschaftsschrifttum des 16. und 17. Jahrhunderts
}

\author{
Philip Habn
}

\begin{abstract}
„Agriculture hath been (not undeservedly) esteemed a Science, that principally teacheth us the Nature, and divers Properties and Qualities, as well of the several Soils, Earths, and Places, as of the several Productions or Creatures, whether Vegetable, Animal, or Mineral, that either Naturally proceed, or are artificially produced from, or else maintained by the Earth."1
\end{abstract}

Mit diesem Satz beginnt eines der bedeutendsten englischen Landwirtschaftslehrbücher des 17. Jahrhunderts, die Systema Agriculturae des John Worlidge von 1675. Es liegt auf der Hand, dass solche Schriften für die Erforschung vergangener Umweltwahrnehmungen aufschlussreich sein können. Dennoch wurden sie lange vernachlässigt. Die klassische Überblicksdarstellung zur Wahrnehmung der Umwelt in der Geistesgeschichte des Westens, Clarence Glackens Traces on the Rhodian Shore, ${ }^{2}$ gedenkt ihrer mit keiner Silbe - nur zwei Dokumente zur Forstwirtschaft des 17. Jahrhunderts finden darin Berücksichtigung. ${ }^{3}$ Schon anders ist dies

\footnotetext{
1 Worlidge J (1681) Systema Agriculturae. Dring, London. S. 1.

2 Glacken T (1967) Traces on the Rhodian shore. Nature and culture in western thought from ancient times to the end of the eighteenth century. University of California Press, Berkeley. S. 484ff.

${ }^{3}$ John Evelyns Silva von 1664 und Colberts Waldverordnung von 1669.
} 
bei Keith Thomas ${ }^{6}$ Man and the Natural World, ${ }^{4}$ das auf breiter Quellenbasis den Wandel des Umweltverhaltens in England zwischen 1500 und 1800 analysiert und dabei vereinzelt auch auf agronomische Werke zurückgreift. ${ }^{5}$ Erst seit einigen Jahren sind diese dann verstärkt in den Fokus umweltgeschichtlicher Forschungen gerückt. So wies Verena Winiwarter darauf hin, dass es sich bei landwirtschaftlichen Handbüchern um „Programme zur Intervention in ökologische Systeme“ handle, deren spezifische Verknüpfung von kulturellen Konzepten und physischen Sinneserfahrungen zu untersuchen seien. ${ }^{6}$ Zusammen mit Martin Schmid hat Winiwarter am Beispiel der Bodenkunde in der Oeconomia Johannes Colers demonstriert, wie eine solche Schrift aus umweltgeschichtlicher Perspektive analysiert werden kann. Sie identifiziert Colers Ausführungen über Bodenqualitäten als „sozionaturalen Schauplatz“, der durch 1. Sinneserfahrung, 2. deren Darstellung als kulturell vermittelbare Inhalte und 3. Umwandlung in Programme sowie schließlich 4. Umsetzung in Arbeit konstituiert werde. ${ }^{7}$ Im Anschluss an dieses Konzept möchte ich im Folgenden den Blick auf Westeuropa ausdehnen und anhand einiger wichtiger landwirtschaftlicher Lehrbücher aus England, Frankreich, Italien, den Niederlanden und dem deutschsprachigen Raum aufzeigen, welche unterschiedlichen Ausprägungen der ,sozionaturale Schauplatz Landwirtschaft" ${ }^{\text {"8 }}$ im europäischen Kontext erfuhr. Dabei konzentriere ich mich vor allem auf die Aspekte der kulturellen Repräsentation und der Handlungsanweisungen.

Bekanntlich erfuhr die gelehrte Auseinandersetzung mit der Landwirtschaft in der Renaissance Aufwind durch die Lektüre der antiken Res-rustica-Literatur. Bald jedoch wurde man sich bewusst, dass die antiken Lehren sich aufgrund klimatischer Unterschiede nicht in ganz Europa realisieren ließen. Aus diesem Grund schufen die französischen Ärzte Charles Estienne und Jean Liébault mit ihrer Maison rustique ein Lehrbuch, das sich bewusst auf die französischen Verhältnisse bezog. Es erschien erstmals 1564 und erlebte bis zum Ende des 17. Jahrhunderts über dreißig Auflagen sowie ihrerseits mehrfach aufgelegte Übersetzungen ins Englische, Italienische, Niederländische und Deutsche (Abb. 1). ${ }^{9}$ Damit war es das einflussreichste Landwirtschaftsbuch Europas. Allerdings machten die Übersetzungen ihrerseits deutlich, dass auch dieses ausdrücklich französische Werk nicht überall gleichermaßen anwendbar war. So stellte Gervase Markham zu Anfang seiner Überarbeitung des Estienne-Liébaultschen Ackerbauwerkes fest, dass Klima und Bodenbeschaffenheit in England „so much remote in nature and qualitie“ von denjenigen Frankreichs seien, dass er sich genötigt sah, die Übersetzung des ur-

\footnotetext{
4 Thomas K (1983) Man and the Natural World. Changing Attitudes in England 1500-1800. Penguin, Harmondsworth.

${ }^{5}$ Ebd. Thomas verwendet u. a. die Lehrbücher von Walter Blith und John Worlidge.

6 Winiwater (2009), S. 228.

7 Schmidt u. Winiwater (2008).

${ }^{8}$ Ebd., S. 171.

9 Estienne (1570), unpag. Epistre dedicatoire: „que les estrangers, comme Flamans, Allemans, Anglois, quasi envieux de ce labeur, ont prins la pleine de le translater en leur langue.“
} 
sprünglichen Textes um die „experience and knowledge of our best Husbandmen“ zu ergänzen. ${ }^{10}$

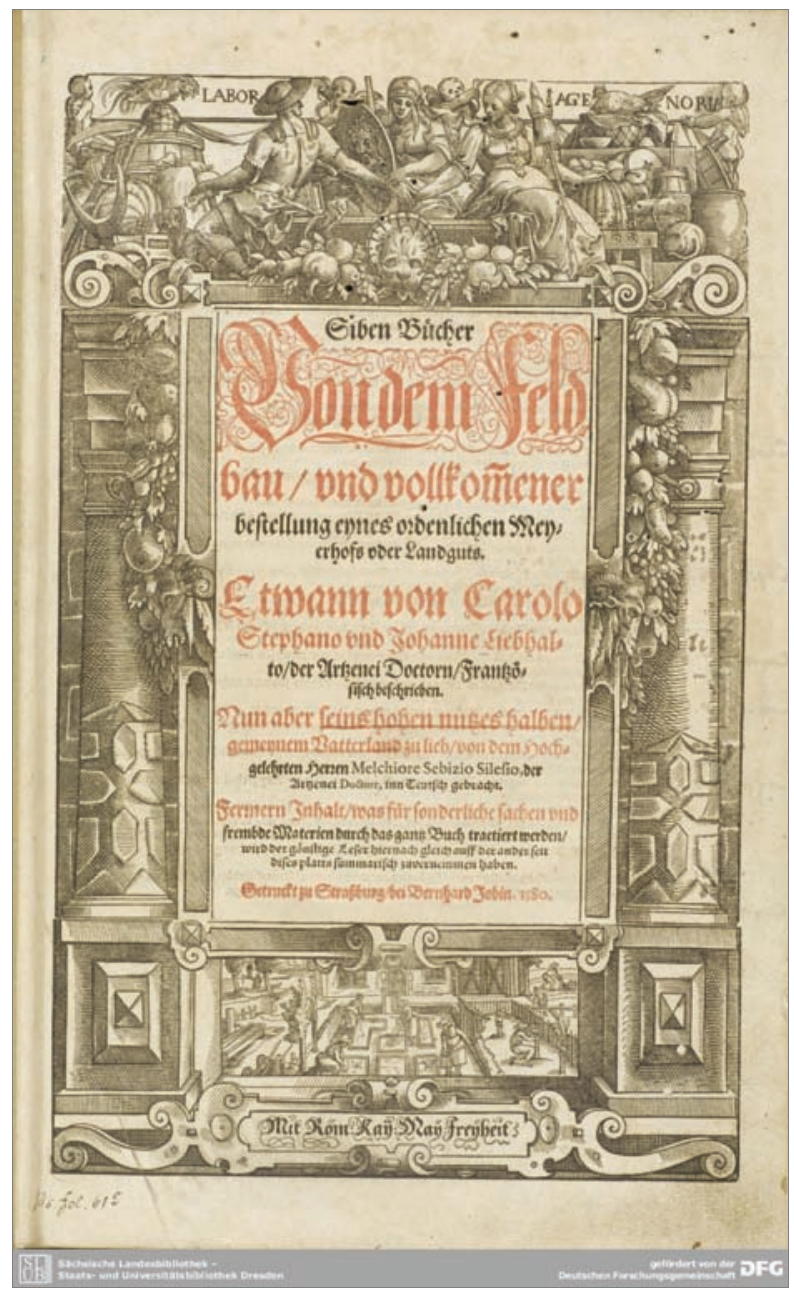

Abb. 1: Deutsche Ausgabe der Maison rustique von Estienne und Liébault (Sächsische Landes- und Universitätsbibliothek Dresden, Sig. 1 B 4058).

Ganz ähnlich widerfuhr es der deutschen Übersetzung des Straßburger Arztes Melchior Sebizius. Hatte dieser bereits bei seiner ersten Auflage einige Kapitel um Hinweise aus deutschen Landen ergänzt, so fügte er 1592 ganze Bücher aus der Feder des kurpfälzischen Leibarztes Georg Marius und anderer Autoren ein und

\footnotetext{
${ }^{10}$ Estienne H (1616) The Countrey Farme. Hrsg. und übs. von G. Markham. Bill/Islip, London. S. 2.
} 
übernahm auch die von Jean Liébault in den letzten französischen Ausgaben vorgenommenen Ergänzungen. Vor allem habe er aber, so heißt es in der Vorrede des Buchdruckers, ,auch sonsten/ was er der Teutschen Landsart zum gemässesten gewußt/ auß mancherley seinen Collectaneis vnnd Obseruationen hievon/ ordentlich beigesetzt" ". ${ }^{11}$ Der italienische Übersetzer der Maison rustique gesteht in seiner Vorrede, dass er bei einigen botanischen Ausdrücken Übersetzungsprobleme gehabt habe, erwähnt aber etwaige Anpassungen an italienische Verhältnisse mit keiner Silbe. Er preist die Vorzüge dieses Werkes und hebt hervor, dass dessen Autor ein Arzt sei und es daher auch ,secreti delle cose della natura, \& della Magia naturale" zu bieten habe. Ein im Anschluss abgedrucktes Gedicht auf den Übersetzer Hercole Cato greift das Thema der ,alti secreti“ der Natur wieder auf: Der Herrscher des Himmels habe Cato dazu auserwählt, diese Geheimnisse zu enthüllen, alle anderen - auch der Italiener Agostino Gallo - hätten nun zu schweigen. Erst die zweite italienische Auflage wurde unter anderem um einen Weinbautraktat ergänzt. Die bis 1627 sechsmal aufgelegte niederländische Ausgabe stellt eine unveränderte Übersetzung aus dem Deutschen dar. Die deutsche Vorlage war allerdings bereits 1607 zum letzten Mal erschienen, denn inzwischen hatte sich ein anderes Werk den deutschen Buchmarkt erobert, nämlich die Oeconomia Johannes Colers. Dieser plädierte genau wie schon Liébault für eine regional zugeschnittene Lehre, denn sein Vater hätte beim Versuch, die Ratschläge der antiken Ackerbauschriftsteller umzusetzen, Schiffbruch erlitten, habe auch

„da er mit Bawren/ Scheffern=Gertnern/ Weinmeistern/ vnd andern vmbgangen/ vnd dieselbigen gehöret vnd ausgeforschet/ ... in vielen dingen gar ein anders befunden“ und damit mehr Erfolg gehabt. Denn, so fasst Coler zusammen, „So ist trawen auch ein grosser vnterscheidt in der Landtart/ darinnen einer wonet/ ein jedlich Landt/ Erdreich/ Bodem/ Acker oder Wiesen hat seine besondere Eigenschafft/ seine Erde/ seine Lufft/ sein Wasser/ etc. darnach sich ein Haußwirt richten mus."12

Colers Werk erlebte dann in der zweiten Hälfte des 17. Jahrhunderts eine niederländische und eine schwedische Bearbeitung, die beide ihrerseits auf die jeweiligen Verhältnisse zugeschnitten waren. ${ }^{13}$

Die Auseinandersetzung zunächst mit antiken, später mit zeitgenössischen ausländischen Landwirtschaftslehrbüchern förderte demnach europaweit ein mehr oder minder stark ausgeprägtes Bewusstsein für die Besonderheiten der jeweiligen Umwelt. ${ }^{14}$ Bemerkenswert ist dabei die unterschiedliche Tiefenschärfe der Diffe-

\footnotetext{
11 Estienne H (1592) XV. Bücher Von dem Feldbaw vnd recht volkommener Wolbestellung eines bekömmlichen Landsitzes. Übs. von M. Sebizius. Jobin, Straßburg. Fol. * 3 r.

${ }^{12}$ Coler J (1591) Calendarium oeconomicum et perpetuum. Hrsg. von G. Frühsorge. Edition Leipzig, Leipzig. Fol. A 3 v.

13 Vgl. Hahn P (2013) Das Haus im Buch. Konzeption, Publikationsgeschichte und Leserschaft der "Oeconomia" Johann Colers (erschienen 1591-1692). Bibliotheca academica, Epfendorf. S. 247-280.

14 Thirsk J (1992) Making a Fresh Start. Sixteenth-Century Agriculture and the Classical Tradition. In: M. Leslie, T. Raylor (ed) Culture and Cultivation in Early Modern England. Leicester UP, Leicester. S. 15-34. S. 28.
} 
renzierung. Bereits Jean Liébault hatte zugeben müssen, dass die französischen Böden sehr unterschiedlich seien und die Maison rustique sich in erster Linie auf die Landwirtschaft der Île de France beziehe; dennoch verstand er sie als ein „exemplaire“ für andere Regionen Frankreichs. Coler setzte sich hingegen mit den Besonderheiten einzelner Regionen des deutschen Reiches auseinander, vor allem mit den ihm persönlich bekannten Landschaften Brandenburgs, Mecklenburgs und Schlesiens. Der englische Estienne-Übersetzer Markham wiederum legte darauf Wert, „our Nation“ (also England) als Ganzes von Frankreich zu unterscheiden, wobei hier auch politisch-konfessionelle Erwägungen eine Rolle spielten. ${ }^{15}$

Eine weitere Gemeinsamkeit fällt auf: die Betonung von „experientia“ bzw. „experience“ sowie „Obseruationen“. Bei Estienne-Liébault heißt es, ein Landwirt müsse nicht „lettré" sein, sondern künftigen Niederschlag und Veränderungen der Windrichtung und Temperatur anhand der Beobachtung der Farbe von Sonne und Mond, dem Verhalten von Vögeln, Insekten und Weidetieren, oder die Fruchtbarkeit eines Jahres anhand der Menge der Früchte auf den Bäumen vorhersagen können. ${ }^{16}$ Coler betont, dass einfache Ackerleute, Gärtner, Weinmeister oder Schäfer „so weder schreiben noch lesen können/ offt mit jhrer schlechten nachrechnung vnd einfeltigem nachdencken/ ex antecedentibus experientijs" in landwirtschaftlichen Dingen eher recht hätten als Gelehrte. ${ }^{17}$ Deshalb habe er sich „Bauern/ Schwein= und Kühhirten die mit diesen Sachen täglich umgangen“" als Lehrmeister gewählt und auch er selbst habe

„die Experientz und Erfahrung mit zu rath genommen/ und bin bißweiln wann ich mich müde gestudiert/ in die Ställe mit zu den Rossen und anderm Vieh gangen/ bin auff dem Felde herum spatziert/ und habe allzeit grosse Lust und Liebe zu lernen gehabt/ nicht allein von Hirten und Bauren/sondern auch von der gantzen Natur/ in libro de natura, darinnen viel treffliche Künste und grosse Geheimnuß verborgen liegen." ${ }^{\text {"18 }}$

Vergleicht man die Positionen der Lehrbücher zur Bodenverbesserung, so ergeben sich signifikante Unterschiede. Estienne und Liébault erklären, dass die auf unbewirtschaftetem Boden wachsenden Kräuter Auskunft über die Bodengüte geben. ${ }^{19}$ Sie klassifizieren den Boden entsprechend der Humoraltheorie (naß - trocken, kalt - heiß), richten aber ihre Empfehlungen zur Düngung mit Mist, Anreicherung mit Mergel oder Besömmerung der Brache mit Leguminosen nicht im Einzelnen danach aus. Nur kurz weisen sie darauf hin, dass nicht nur die Art der Bearbeitung,

\footnotetext{
${ }^{15}$ Estienne H (1567) L'Agriculture et Maison rustique. Du-Puys, Paris. S. 196. Zu Coler s. Winiwarter V, Schmidt M (2008) Umweltgeschichte als Untersuchung sozionaturaler Schauplätze? Ein Versuch, Johannes Colers „Oeconomia“ umwelthistorisch zu interpretieren. In: T. Knopf (ed) Umweltverhalten in Geschichte und Gegenwart. Attempto, Tübingen. S. 158-173. Zu Markham s. Wall W (1996), Renaissance National Husbandry. Gervase Markham and the Publication of England. In: Sixteenth Century Journal 27.3. S. 767-785.

${ }^{16}$ Estienne (1567), S. 11f. u. Estienne H (1580) Siben Bücher Von dem Feldbau/vnd vollkommener bestellung eynes ordenlichen Meyerhofs. Ubbs. von M. Sebizius. Jobin, Straßburg. S. 40f.

${ }^{17}$ Coler J (1680) Oeconomia ruralis et domestica. Schönwetter, Frankfurt a. M. Teil 1, S. 449.

18 Vgl. hierzu Blumenberg H (1986) Die Lesbarkeit der Welt. Suhrkamp, Frankfurt a. M.

${ }^{19}$ Estienne u Liébault (1580), S. 481-484.
} 
sondern auch die Form des Pfluges der Bodenbeschaffenheit angepasst sein müsse. ${ }^{20}$ Das zweite große französische Ackerbauwerk, Olivier de Serres ${ }^{6}$ Théâtre de l'Agriculture (1603), 1600 erstmals erschienen und bis ins 18. Jahrhundert fünfzehnmal aufgelegt, lässt hier schon eher ein Programm erkennen. Unterschiede in der Bodengüte zu leugnen sei wider den gemeinen Menschenverstand. Auch verlange Gott, dass man sich mit dem Boden zufrieden gebe, den man besitze. Doch empfiehlt de Serres dem ,bon Pere-de-famille“, mit Kunst und Sorgfalt das beste daraus zu machen und den Mängeln abzuhelfen. Dafür bietet er genaue Anleitungen. Ganz ähnlich beurteilen Winiwarter und Schmid die Ratschläge des Deutschen Johannes Coler; ${ }^{21}$ sie zielen nicht auf „Maximierung von Veränderung“ $a b$, sondern auf „Optimierung bestehender Verfahren durch Anpassung an lokale naturale Faktoren wie Wetter und Boden. Einen völlig anderen Eindruck vermittelt die englische Literatur des 17. Jahrhunderts. ${ }^{22}$ Der bereits erwähnte Gervase Markham verfasste auch eine kurze Schrift mit dem Titel The Inrichment of the Weald of Kent, von 1625 bis 1695 zwölfmal aufgelegt, in der er sich detailliert mit den Bodenbeschaffenheiten der Grafschaft auseinandersetzt und den Einsatz von Mergel erörtert. ${ }^{23} \mathrm{Ab}$ der Mitte des Jahrhunderts kann man geradezu eine Sprache des „Agrarian Improvement“ identifizieren, die die englischen Lehrbücher durchzieht. ${ }^{24}$ Während in Frankreich und Deutschland die vor oder um 1600 entstandenen Werke immer neu aufgelegt wurden und erstaunlicherweise auch in den niederländischen Lehrbüchern keine Innovationen vermittelt wurden, ${ }^{25}$ rüsteten sich die englischen Autoren zu einer durchgreifenden Melioration ihres Landes. So verspricht Walter Blith schon auf dem Titelblatt seiner Schrift The English Improver von 1649 eine Verdopplung bis Verzwanzigfachung des Ertrags. ${ }^{26}$ Gleichzeitig warnt er vor falschen Versprechungen etwa von durchs Land ziehenden holländischen Experten. Zunächst geht es ihm darum, die Gründe für Unfruchtbarkeit zu identifizieren und schädliche Praktiken und Gewohnheiten abzuschaffen. Von seinem umfangreichen Maßnahmenkatalog - Schädlingsbekämpfung, Pflügen, Gewässerbegradigung, Aufforstung, um nur einige zu nennen - verspricht er sich,

\footnotetext{
20 Estienne (1580), S. 479.

21 Winiwarter u Schmid (2008), S. 170.

22 Vgl. aber noch die Position von Tusser T (1570) Five Hundred Points of Good Husbandry. Hrsg. von G. Grigson. Oxford UP, Oxford, New York. die mit denjenigen Colers und de Serres` vergleichbar ist.

23 Thirsk J (1983) Plough and pen. Agricultural writers in the seventeenth century. In: T. H. Aston et al. (ed) Social relations and ideas: essays in honour of R. H. Hilton. Cambridge UP, Cambridge. S. 295-318. S. 303-306.

24 McRae A (1992) Husbandry Manuals and the Language of Agrarian Improvement. In: M. Leslie, T. Raylor (ed) Culture and Cultivation in Early Modern England. Leicester UP, Leicester. S. 35-62.

25 Dort wurden v. a. die Übersetzungen der Maison rustique und Colers sowie Groen J, Nyland P (1670) Den Nederlandtsen Hovenier. Den Ervaren Huys-Houder. Doornick, Amsterdam. aufgelegt. Letzteres weist zwar in der Inleidingh darauf hin, „Doch, om tot ons voornemen te komen, seggen wy, dat de Natuur, die sich veeltijts wanschickelijck vertoont, door de konst kan op-geschickt, opgepronckt, in goede ordre, cierlijck en vermakelijck gemaeckt worden“, doch findet dieses Thema im Buch selbst keine weitere Erörterung.

${ }^{26}$ Blith W (1649) The English Improver. Or a New Survey of Husbandry. Wright, London. S. 2.
} 
die Böden in ihrer äußersten ursprünglichen Fruchtbarkeit wiederherzustellen. Sein Credo ist: „All sorts of Lands, of what nature or quality soever they be, under what Climate soever, of what constitution or condition soever, of what face or character soever they be ... will admit of a very large Improvement". Die alten englischen Übersetzungen der Maison rustique taugten dafür allerdings gar nicht, sie enthielten nur ,either Rarely, or Mystically, any Improvement to purpose for this Kingdome“ “.27 1675 formuliert John Worlidge dann etwas vorsichtiger, dass nicht der geringste Teil der Qualifikation eines Landwirtes darin bestehe, herauszufinden, welcher Boden wie bewirtschaftet werden könne, doch verkündet auch er, dass beinahe alles Land ,that bears the name of barren Land, although for the most part by good Husbandry it may be reduced into Tillage, and become very fruitful and advantageous to the Husbandman in particular, and Commonwealth in general“", was durch zahlreiche zeitgenössische Erfolge bewiesen würde. ${ }^{28}$

Diese englischen Schriften lassen aber auch ein Bewusstsein für bestehende Über- oder Fehlnutzungen der Umwelt erkennen. Bereits Thomas Tusser hatte in seinen populären, 1570 zum zweiten Mal erschienenen Five Hundred Points of Good Husbandry davor gewarnt, die Allmende zu sehr abzuweiden: „overlay not thy ground", rief er seinen Lesern zu. ${ }^{29} 1649$ war das Problem noch nicht gelöst, und Walter Blith brachte es erneut auf den Tisch: ,mowing Ground till it Graze no more, or yeeld no grasse; all which are easily to be remedied if men would learne moderation““.30 Ebenso beklagte er die Abholzung „of the gallant Timber of the Kingdom" und verweist auf die nützliche Gesetzgebung in anderen Königreichen, die bei jeder Fällung zur Anpflanzung der zwei- oder dreifachen Anzahl Bäume verpflichte. Worlidge kennt ein vergleichbares Gesetz aus Burgund und verweist zudem auf eine in der Wetterau verbreitete Verordnung, dass eine Heirat erst erlaubt sei, wenn der Bräutigam nachweisen könne, eine bestimmte Anzahl von Walnussbäumen gepflanzt zu haben. ${ }^{31}$ Auch in der zeitgenössischen deutschen Landwirtschaftsliteratur wird dieses Anliegen vorgebracht. Johannes Coler vermahnt die „Weltliche Obrigkeit“ unter seinen Lesern, dass sie ihre Bauern dazu anhalten sollten, jedes Jahr mindestens sechs bis zwölf neue Bäume in ihren Gärten zu pflanzen: „man mus das grobe Volck mit grossem ernst dazu halten/ daß sie Obstbäwme zeugen. Denn dadurch ein Land trefflich an Narung kan wachsen und zunemen“.32 Es ist ihm ferner bewusst, dass die Waldbestände vielerorts ,vergangen vnd verwüstet worden“. Wie man sie neu aufforsten könne, das erfahre man unter anderem von den „Heideleuten“, womit er die Forstarbeiter meint, „die täglich mit solchen dingen vmbgehen". Coler beendet seine Ausführung mit dem Hinweis auf eine mecklenburgische Herzogin, die feierlich mehrere Aufforstungen

\footnotetext{
27 Ebd., S. 20.

28 Worlidge (1681), S. 34, 37.

${ }^{29}$ Tusser (1570), S. 99, 107.

${ }^{30}$ Blith W (1649), S. 12.

31 Worlidge (1681).

${ }^{32}$ Coler J (1632) Calendarium Perpetuum Et Viginti Libri Oeconomici. Helwig/Fincel, Wittenberg. S. 138.
} 
mit Kiefern veranlasst habe und deren Exempel etliche Adlige und Städte nachgefolgt seien. ${ }^{33}$ In den 1682 erstmals erschienenen Georgica curiosa Wolf Helmhards von Hohberg findet sich ein Rat, der an die Maximen der englischen Improvers erinnert, hier jedoch auf die Aufforstung angewandt. ${ }^{34}$ Vielerorts herrsche Holzmangel, was zumeist der „Nachlässigkeit der Inwohner“ anzulasten sei. Schließlich, so Hohberg, gebe es kein Land, das, wenn es Korn trägt, nicht auch Bäume ernähre: „denn die Gegend sey wie sie wolle/ hoch/ nieder/ gebürgig/ steinicht/ thalicht/ feucht oder trocken/ so gibt es doch gewisse Bäume/ denen man daselbst ihren Stand und Platz anweisen kan“. In einem anderen Teil des Werks empfahl der Autor allerdings, Büsche, Nussbäume, Ulmen und Eichen an den Feldrändern und auf den Fluren selbst restlos zu entfernen. ${ }^{35}$ Der eben zitierte Walter Blith setzte sich vehement für die Entwässerung von Feuchtgebieten und die Begradigung von Bachläufen ein. ${ }^{36}$

Die Warnungen Colers vor der Zerstörung von Fischbeständen im Buch Von den Fischen seiner Oeconomia verweisen auf einen bislang noch nicht zur Sprache gekommenen Einflussfaktor auf frühneuzeitliche Umweltwahrnehmungen, nämlich die Religion. Coler schreibt hier von Ereignissen aus seiner Wahlheimat Mecklenburg, bei denen zumindest seiner Ansicht nach Gott die Menschen durch die Natur für ihre Sünden bestraft hat. ${ }^{37}$ Das „Land zu Meckelburg“ und einige andere um die Ostsee herum liegenden Länder hätten „Gott auch viel der Fischerey halben zu dancken/ dann sie haben treffliche/ herrliche/ grosse Seen“, die „überaus fischreich“ seien. Doch sieht sich Coler jedoch genötigt, davon zu berichten, wie sich die Menschen an diesem Fischreichtum versündigt hätten. Die Einwohner der Stadt Röbel bei Malchow beispielsweise hätten „offt viel vnd grosse Murenen gefangen“, so dass sich die Leute „derselben gar vberdrüssig gessen/ vnd endlich eckel darvor gehabt haben." Hierzu weiß der Autor eine Historiam zu erzählen:

\begin{abstract}
„Als sie aber dermal eins derselben auch so gar viel gefangen/ haben sie eine Murena an den Kack genagelt/ vnd haben sie daran gestriechen. Derentwegen sie Gott hernachmalen so gestraffet/ daß sie keine mehr haben fangen können. Was geschicht? Es lest ein guter Mann eine silberne Murenam machen/ vnd verehret sie in die Kirche. Aber es hilfft nicht/ Gott wil kein Zeichen thun. Endlich nimbt der Superintendens desselbigen Orts/ als er da visitiret, die silberne Muhren/ schicket sie ins Hospital/ vnd verehret sie den Armen Leuten/ da gibt Gott widerumb Muhrenen.“38
\end{abstract}

\footnotetext{
33 Ebd.

${ }^{34}$ Hohberg WH v. (1701a) Georgica curiosa aucta. Bd. 1. Endter, Nürnberg. S. 656.

35 Hohberg WH v. (1701b) Georgica curiosa aucta. Bd. 2. Endter, Nürnberg. S. 14.

36 Blith W (1649); vgl. auch Hohberg (1701b), S. 16

37 Vgl. hierzu auch (Coler 1680 S. 183) im Buch Vom Säewerck, wo Coler darauf verweist, dass nicht nur Gott die Menschen durch Mücken und Fliegen strafe, sondern auch der Teufel sich ihrer bediene, , dann wir haben vorm Jahr gesehen und erfahren/ wie der Teuffel den Leuten zu Spandau bißweilen in der Gestalt eines Hauffen grosser Flieben erschienen."

38 Coler J (1640) Colerus redivivus, Sive Oeconomia Universalis Cum Calendario Perpetuo. Schönwetter, Frankfurt a. M. S. 504.
} 
Eine ähnliche Strafe hätten auch die Rostocker erlitten, wie Coler gleich weiter berichtet, die „so viel Dorsen gefangen ... daß sie dieselbigen auch den Schweinen vorgeschütt: Derwegen sie auch Gott gestrafft/ daß sie hernach keine mehr haben fangen können." Aus diesen Geschichten zieht er nun eine allgemeingültige Moral für seine Leser: „Dann Gott wil/ daß man seine Geschenck und Gaben mit danckbarem Hertzen erkennen und annehmen/ und keines weges mißbrauchen soll.“"39 Überhaupt ist Colers ganze Oeconomia geistlich durchdrungen; besonders deutlich wird das bei den Themen Ackerbau, Weinbau und Viehzucht. Letzterer schickt er eine ganze Reihe von Bibelzitaten voraus, die sowohl die Herrschaft des Menschen über das Tierreich begründen als auch ihn an seine Verantwortung vor Gott erinnern (darunter auch einige obskure Stellen wie Deuteronomium 22,4: „Du solt dem Ochsen/ der da drischet/ das Maul nicht verbinden“). Auch der Franzose Olivier de Serres ermahnt seine Leser, dass man durch sorgfältigen Ackerbau dem Boden reiche von Gott gegebene Schätze entziehe, und daher müsse man alles daran setzen, diese zu seiner Ehre einzusetzen, „pour vser, \& non abuser de ses biens". .40

Der Improver Blith erinnert daran, dass der ursprüngliche Grund von Unfruchtbarkeit des Bodens bei dem Menschen selbst liege, ,who by his sinne procured a curse upon the Land". ${ }^{41}$ Ansonsten spielt die Religion bei ihm allerdings eher eine unterstützende Rolle, etwa indem er Gott als „first Husbandman“ bezeichnet, der, indem er das Chaos geordnet, ein unermessliches „Improvement“ vollbracht habe. Ferner zitiert er Ermahnungen aus der Heiligen Schrift zum Fleiß in der Landwirtschaft. ${ }^{42}$ Eine ausgesprochen religiös motivierte Perspektive auf die Umwelt offenbart auch Wolf Helmhard von Hohberg, der in seiner Einführung in den Gartenbau daran erinnert, dass Gott selbst „die erste Anweisung und Anleitung“ hierzu getan hätte. ${ }^{43}$ Die Gartenarbeit sei also mitnichten eine

„Wirckung des Göttlichen Fluchs/ sondern eine Lust-Übung der Gesundheit/ eine Nachahmung der Göttlichen Natur/ ein Spiegel des künfftigen Paradises/ eine Erquickung der abgematteten Geister/ eine Lust-Arbeit der menschlichen Begierden/ eine kleine Academie des Glaubens/ der Liebe/ der Hoffnung/ der Gedult ${ }^{\star 44}$

und so fort. Außerdem habe Christus gern in Gärten gebetet und sei nach seiner Auferstehung der Maria Magdalena als Gärtner erschienen. Ferner führt Hohberg in seiner Wasser-Lust die „Nutzbarkeit des Wassers“ unter anderem mit dem Hin-

\footnotetext{
${ }^{39}$ Coler (1680), S. 650f.

40 Serres O de (1603) Le Theatre d'Agricvltvre et Mesnage des Champs [...] Seconde Edition. Saugrain, Paris. S. 741, S. 904.

41 Blith W (1649), S. 7.

42 Ebd., S. $5 f$.

43 Hohberg (1701a), S. 528.

44 Vgl. Frühsorge G (1986), Georgica curiosa'. Vom geistlichen Sinn der Anleitungsliteratur bei Wolf Helmhard von Hohberg. In: H. Zeman (ed) Die österreichische Literatur. Ihr Profil von den Anfängen im Mittelalter bis ins 18. Jahrhundert (1050-1750), Teil 2. Akademische Druck- und Verlagsanstalt, Graz. S. 1071-1086.
} 
weis auf die Taufe mit Wasser vor Augen. ${ }^{45}$ Auch dieser religiöse Aspekt der Umweltwahrnehmung in der Landwirtschaftsliteratur kann also durchaus als ein europäisches Phänomen betrachtet werden.

Doch noch einmal zurück zu Hohberg. Im Blumen-Garten erklärt derselbe Autor das rasche Verwelken der Schönheit der Blumen damit, dass ,der sündliche Undank der Menschen-Kinder/ einer so liebreichen Begünstigung seiner Gütigkeit nicht würdig“" sei, da er nur seiner Augenlust nachtrachte, anstatt sich zu Herzen zu nehmen, dass der Mensch einer Blume gleiche, die ebenso schnell umgeblasen werden könne, „damit er von diesen kleinen und holdseligen Schulmeistern weiser werden und mit mehr und tiefferm Nachsinnen an seine Sterblichkeit zu eigener Wolfahrt gedencken“ möchte. ${ }^{46}$ Dieser Aspekt der Umwelt, gleichsam als Natura magistra vitae zu fungieren, wird auch von Olivier de Serres unterstrichen, allerdings mit einer ganz anderen Zielrichtung: ${ }^{47}$ Der Boden selbst klage diejenigen der Nachlässigkeit an, die es nicht zustande bringen, ihm das zu entlocken, was in seinem Innersten angelegt sei. Klassisch ist aber vor allem das Exempel der Bienen. In die frühneuzeitliche Landwirtschaftsliteratur wurde es allerdings erst von Johannes Coler eingeführt, denn sowohl die Bienenkapitel bei Petrus de Crescentiis als auch bei Estienne und Liébault verraten nur ansatzweise eine allegorisierende Tendenz. ${ }^{48}$ Coler hingegen beschreibt den Bienenstaat, seine Herrschaftsform und Arbeitsteilung fast durchgängig mithilfe menschlicher Analogien. ${ }^{49}$ Auch lässt er erst gar keinen Zweifel aufkommen, was er sich davon erhofft, denn er mahnt, „ein jeder frommer Haußwirth“ solle Bienen nicht nur des Honigs halber halten, sondern auch wegen ihres

„wolbestellten Regiment[s]/ daß sie uns in vielen Sachen gute Exempel und instruction geben/ wie wir uns im gemeinen bürgerlichen Leben und in unserer Haußhaltung verhalten sollen/ wann wir nur ihrem Exempel/ Thun und Wesen recht nachdencken und nachfolgen wollen". ${ }^{0}$

Denn abgesehen von ihrer mustergültigen politischen Ordnung werden bei Coler die Bienen auch als Vorbild individueller Lebensführung dargestellt. ${ }^{51}$ So schreibt

\footnotetext{
45 Hohberg (1701b), S. 521.

46 Hohberg (1701a), S. 740.

47 De Serres (1603), S. 903.

48 Crescentiis P de (1538) De Agricultura, Omnibusque Plantarum, \& animalium generibus. Petri, Basel S. 486f. Dessen dt. Übs.: Crescentiis P de (1602) New Feldt und Ackerbau [...] von newem gemehrt worden [...] vnd durch einen Hochgelehrten deß Feldbawes wol erfahrnen/ vor Jahren in vnser Teutsche sprach an Tag gebracht. Zetzner, Straßburg. S. 490-503, ist identisch mit Estienne 1592, S. 392-407 (beide übersetzt von dem Straßburger Stadtphysikus Melchior Sebizius). Zu Coler s. Hahn (2013). Zum Bienenexempel allgemein s. Peil D (1983) Untersuchungen zur Staats- und Herrschaftsmetaphorik in literarischen Zeugnissen von der Antike bis zur Gegenwart. Fink, München. Peil benutzt Coler (1680), S. 196, 205, 207, 216, 235, 237-40, 244, 270. S. a. Wimmer E (1998) Biene und Honig in der Bildersprache der lateinischen Kirchenschriftsteller. Österreichischer Kunst- und Kulturverlag, Wien; Thomas 1984, S. 62-63.

49 Coler (1680), S. 545.

${ }^{50} \mathrm{Vgl}$. Thomas (1984), S. 64.

51 Coler (1680), S. 546.
} 
er an einer Stelle: „Daher es dann auch kombt/ daß diese kleine Thierlein so lang leben können/ nemlich daß sie fleissig arbeiten/ zu rechter Zeit schlaffen gehen/ und von der Frauen Venere nichts wissen." 52 Dennoch hat das Vorbild der Bienen auch seine Grenzen: Eine Frauen- und Kindergemeinschaft, wie sie in Platons Staat von den Bienen abgeleitet wird, lehnt Coler ab, denn „Gott hat uns in seinem Wort ein anders gelehrt." 53 Auch im frühneuzeitlichen England hatte das Bienenexempel große Konjunktur, allerdings - aufgrund der sich hier früh durchsetzenden Ausdifferenzierung der landwirtschaftlichen Literatur ${ }^{54}$ - in der apiologischen Fachliteratur, und nicht in den Landwirtschaftslehren. Die Interpretation des Bienenstaates wurde während der Englischen Revolution so sehr zum politischen Fußball - Monarchie der Bienen versus Commonwealth des Bienenstocks - dass einer der bedeutendsten Agrar- und Gartenbaureformer der Zeit, Samuel Hartlib, bewusst darauf verzichtete, die monarchische Analogie im Detail auszuführen, weil er befürchtete, dass sein Plädoyer für eine Ausweitung der Bienenhaltung damit auf Ablehnung stoßen würde. ${ }^{55}$

Bienen verlangten den Lehrbüchern des 16. und 17. Jahrhunderts zufolge auch eine besondere Behandlung. Da ihr ,,verstand vnd vorsichtigkeyt“ denjenigen des Menschen nahekomme, heißt es bei Estienne und Liébault in der deutschen Übersetzung, dürfe ein Imker ,keyn betrieglicher/ vnsauberer/ noch vnfleissiger vnd liederlicher/ sondern eyn auffrichtiger/ redlicher/ sauberer vnd Emsiger Man sein. Dann die Binen mögen keynes wegs leiden/ das man mit jnen vnsauber oder zu sparsam vnd zu geitzig vmbgehe“. Sein Amt sei es in erster Linie, darüber Bescheid zu wissen, „was weiß vnd natur/ leben/ thun vnd lassen der Binen seie/ vnd er selbs sich auch auff das aller schleinichst wisse darein zu schicken“.56

Vergleichbares findet sich auch bei Coler wie auch, dass Bienen keine Unkeuschheit ihres Halters oder ihrer Halterin dulden. ${ }^{57}$ Bei Coler trifft man immer wieder auf Plädoyers für artgerechte Tierhaltung, wohlbemerkt, auf der Basis seiner Wahrnehmung der Natur der betreffenden Tiere. So sei das Pferd „das aller edelste vnd nützlichste“ aller vierfüßigen Tiere und außerdem ,ein reinlich Thier/ und will fein sauber und rein gehalten seyn", daher habe man die Ställe täglich zu reinigen. Auch schimpft er über die Brutalität der Fuhrleute, denn Zugpferde sollten nicht geschlagen, sondern „fein freundlich“ ermutigt werden. ${ }^{58}$ Die Gans hingegen sei zwar Coler zufolge ein „tölpischer/ alberer/ einfältiger unnd ungelehriger Vogel“, doch auch hier gebe man Acht, dass man sie stets zu mehreren gemeinsam halte, da Gänse ,allezeit beysammen seyn wollen/ und die

52 Vgl. Hohberg (1701b), S. 416-417.

53 Coler (1680), S. 545.

54 Hierzu s. Thirsk (1992), S. 21.

55 Vgl. hierzu Raylor T (1992) Samuel Hartlib and the Commonwealth of Bees. In: M. Leslie, T. Raylor (ed) Culture and Cultivation in Early Modern England. Leicester UP, Leicester. S. 91-129. S. 112.

56 Estienne (1580), S. 299; vgl. Estienne H (1600) Maison Rustique. Or The Covntrie Farme. Übs. von R. Surflet. Bollisant/Norton, London. S. 407.

${ }^{57}$ Coler (1632), S. 329; Coler (1680), S. 528.

58 Coler (1632), S. 355. 
Gemeinschafft lieb haben".59 Besonders bemerkenswert ist jedoch seine Anweisung an die Schafhirten. Sie sollten „,vorsichtig seyn/ vnd die Schaf lieb haben/ jhr bestes suchen“ und mit ihnen „fein gelinde vmbgehen/ Vnd sie stetigs mit pfeiffen/ züschen vnd singen belustigen vnd erfrewen", denn das Schaf sei ein Tier, „das grossen gefallen an der Musica hat". Er kritisiert daher die heimischen Hirten, die keine Sackpfeife dabei hätten, um den Schafen ein „Geistlich Lied“ zu spielen, wie dies andernorts üblich sei. ${ }^{60}$

Das Vergnügen der Menschen an der Umwelt ist ebenso ein wichtiges Thema in der europäischen Landwirtschaftsliteratur der frühen Neuzeit, hier ließen sich zahlreiche Beispiele anbringen. Erwähnt sei nur, was der internationale Bestseller, die Maison rustique, zu den „lustbarkeyten eynes Gehöltzes“ zu sagen hat: Nach Möglichkeit solle man einen Wald nahe beim Landgut anpflanzen - hier ist wohlbemerkt von „,wildem gehöltz“ die Rede, dessen Bäume jedoch am besten im Abstand von vier mal vier Fuß zu setzen seien, denn das erfreue das Auge und biete später die Möglichkeit, überall gerade hindurch zu fahren. ${ }^{61}$ Durch das vielfältige Grün werde „das gesicht vnd das hertz erlustigt“, der „liebliche vnd holdselige Vogelgesang vnd Melodei“ insbesondere der Nachtigall sei „eyne sondere hertzenfräud auß dem hause zuhören/ welches nahe an eynem gehöltzlin steht gebauen": Das Haus soll also den Autoren zufolge nicht von der Natur durch einen geometrisch geordneten Garten abgeschirmt werden, wie dies dann wenig später allenthalben üblich wird, ${ }^{62}$ sondern zumindest deren Geräusche dürfen bis ins Haus selbst vordringen. Des Weiteren biete ein Gehölz im Sommer kühle Luft und im Winter Schutz vor rauen Winden, und obendrein genügend Tiere zur Jagd „für kurtzweil“" und „zur täglichen Kuchenspeise“ - ein wahrhaft alle Sinne befriedigendes Erlebnis also. ${ }^{63}$ Einige spätere französische Ausgaben dieses Lehrbuches enthielten zudem ausführliche Kapitel über die Haltung von Singvögeln, in denen unablässig von der Freude an ihrem Gesang die Rede ist und sogar erläutert wird, wie man stumme Vögel zum Singen bringe. Diese Kapitel entfalteten jedoch kaum europäische Wirkung, denn nur in der englischen Ausgabe von 1600 sind sie ebenfalls anzutreffen, wurden jedoch 1616 in der Bearbeitung von Gervase Markham herausgekürzt - der Landverbesserer hatte für diese keinen greifbaren Ertrag bringenden Vergnügungen wohl keinen Sinn. ${ }^{64}$

Dass diese Seite der Umweltwahrnehmung ganz klar der ständischen Ordnung der zeitgenössischen Gesellschaft unterlag, braucht wohl kaum hervorgehoben zu

\footnotetext{
${ }^{59}$ Coler (1680), S. 488, 495.

${ }^{60}$ Coler (1632), S. 428.

${ }^{61}$ Estienne (1600), S. 811; Estienne (1580), S. 547.

62 Vgl. die zahlreichen Abb. in Groen/Nyland 1670.

${ }^{63}$ Estienne (1580), S. 547.

${ }^{64}$ Estienne (1600), S. 879ff.; vgl. Estienne (1653); nicht enthalten in: Estienne H (1570) L'Agriculture et Maison rustique. Du-Puys, Paris.; Estienne H (1588) De Veltbouw ofte Lantwinninghe: inhoudende eene rechte wel bestellinghe eenes hofs te bouwen. Claesz, Amsterdam; Estienne H (1606) Agricoltvra nvova, et casa di villa di Carlo Stefano. Übs. von H. Cato. Valentin, Venedig; Estienne (1616). Gleichwohl spricht Worlidge (1681) in der Vorrede von den „Pleasures und Oblectations“ der Natur, der „sweetness and beauty of the Flowers" usw.
} 
werden. ${ }^{65}$ So weist die Widmung der zweiten italienischen Auflage des EstienneLiébaultschen Werkes an den Herzog von Savoyen darauf hin, dass darin genau die Dinge behandelt würden, an denen er sich erfreute, wenn er sich von den schweren Amtsgeschäften in seinen Park zurückziehe. ${ }^{66}$ Insbesondere gilt das natürlich für die zumeist nur dem Adel erlaubte Jagd. Der Landadlige Hohberg setzt sich in seinem Lehrbuch besonders gründlich mit Jagdprivilegien auseinander und bringt bei der Gelegenheit unter anderem folgendes aus umwelthistorischer Perspektive interessante Argument vor: Das Wild müsste nämlich, „wann es Jederman frey wäre/ und zur Zeit und Unzeit von so vielen aufgesucht und gefället würde/ endlich gar ausgerottet werden/ welches nicht zu gestatten/ weil dessen ohne diß sehr wenig ist". .77

Für die beiden prominentesten Vertreter der deutschen Landwirtschaftsliteratur der Zeit, Coler und Hohberg, bot das Thema der Jagd allerdings auch etwas, das nicht nur Standes-, sondern auch Vorstellungsgrenzen überstieg: nämlich die fremde, bedrohliche Umwelt, die sie nur vom Hörensagen oder aus sagenhaften Berichten kannten. Beide konnten sich nicht enthalten, über die teils abenteuerliche Jagd von Löwen, Tigern, Elefanten und Affen zu schreiben, doch damit nicht genug: Coler erzählt ausführlich über livländische Werwölfe, ${ }^{68}$ und Hohbergs Buch enthält sogar ein Kapitel Von dem Drachen. ${ }^{69}$ Lange habe er nicht an deren Existenz glauben können, doch habe ihn Athanasius Kircher eines besseren belehrt. Kirchers Theorie über deren Entstehung sowie einige "glaubwürdige Exempla“ von Begegnungen mit Drachen gibt er daher an seine Leser weiter. Seine Formulierung, dass die ,grossen Berg-Grufften und Höhlen/ in den grossen Schweitzerischen und Carpatischen ausgeödten und abgelegenen Gebürgen [...] noch zu Zeiten von diesen Monstris nicht befreyet" seien, deutet aber an, das diese Welt auf lange Sicht dem Untergang geweiht ist. ${ }^{70}$

Die europäische Landwirtschaftsliteratur - zumindest die hier betrachteten Werke - ist aus der Wahrnehmung regionaler Umweltbedingungen entstanden, und Sinneswahrnehmungen sowie gesammelte Erfahrungen in und mit der jeweiligen Umwelt spielten in den meisten Werken eine bedeutende Rolle. Allerdings wurde die Wahrnehmung der Autoren unverkennbar durch kulturell geprägte Muster beeinflusst: Religion, Moral, Gesellschaftstheorie und Standesdenken bestimmen bis ins 18. Jahrhundert hinein das Bild der Umwelt der Landwirtschaftsliteratur, und damit auch ihrer Leser. Denn es handelte sich hierbei um alles andere als

\footnotetext{
${ }^{65} \mathrm{Zu}$ diesem Thema generell s. Schmidt-Voges I (2008) Oíko-nomía. Wahrnehmung und Beherrschung der Umwelt im Spiegel adeliger Haushaltungslehren im 17. und 18. Jahrhundert. In: H. Düselder et al. (ed) Adel und Umwelt. Horizonte adeliger Existenz in der Frühen Neuzeit. Böhlau, Köln, Weimar, Wien.

${ }^{66}$ Estienne H (1609) Agricoltvra et casa di villa di Carlo Stefano. Übs. von H. Cato. Tarino, Turin.

${ }^{67}$ Hohberg (1701b), S. 684.

${ }^{68}$ Coler (1632), S. $500 f$.

${ }^{69}$ Hohberg, (1701b), S. 698-705.

${ }^{70}$ Ebd., S. 703.
} 
eine nur „virtuelle Landwirtschaft“, wie Jörn Sieglerschmidt formulierte. ${ }^{11}$ Forschungen zu den englischen Ackerbaulehren und für den deutschsprachigen Raum zu Colers Oeconomia belegen, dass diese Bücher tatsächlich in der Praxis angewandt wurden: Ihre „Programme“ gelangten damit zur Umsetzung in „Arbeit“.72 Auch wenn seit der Mitte des 16. Jahrhunderts durch Übersetzungen und Bearbeitungen der Lehrbücher bis zu einem gewissen Maße ein europaweiter Austausch landwirtschaftlichen Wissens stattgefunden hat, so wird spätestens um die Mitte des 17. Jahrhunderts ein wachsender Unterschied der „sozionaturalen Schauplätze“ deutlich: Während in Deutschland die von Coler vertretene, in eine Verhaltensethik eingebettete ,ganzheitliche Praxis“ noch gegen Ende des Jahrhunderts von Hohberg fortgesetzt wird, ${ }^{73}$ legen die zeitgenössischen englischen Improvers den Grundstein zu einer rein auf maximale Ertragsteigerung ausgelegten Landwirtschaft (man denke an das Titelblatt des Buches von Walter Blith). Kein Wunder also, dass die englischen Agronomen dann im 18. Jahrhundert federführend sind. Der Zugewinn an Wissenschaftlichkeit ging allerdings einher mit einem Verlust der Vielfalt frühneuzeitlicher Umweltwahrnehmungen.

\section{Literatur}

Blith W (1649) The English Improver. Or a New Survey of Husbandry. Wright, London.

Blumenberg H (1986) Die Lesbarkeit der Welt. Suhrkamp, Frankfurt a. M.

Coler J (1632) Calendarium Perpetuum Et Viginti Libri Oeconomici. Helwig/Fincel, Wittenberg.

Coler J (1640) Colerus redivivus, Sive Oeconomia Universalis Cum Calendario Perpetuo. Schönwetter, Frankfurt a. M.

Coler J (1680) Oeconomia ruralis et domestica. Schönwetter, Frankfurt a. M.

Coler J (1591) Calendarium oeconomicum et perpetuum. Hrsg. von G. Frühsorge. Edition Leipzig, Leipzig.

Crescentiis P de (1538) De Agricultura, Omnibusque Plantarum, \& animalium generibus. Petri, Basel.

\footnotetext{
${ }^{71}$ Sieglerschmidt J (1999) Die virtuelle Landwirtschaft der Hausväterliteratur. In: R. P. Sieferle (ed) Natur-Bilder: Wahrnehmungen von Natur und Umwelt in der Geschichte. Campus, Frankfurt, New York. S. 223-254.

72 Winiwarter V (2009) 'Haereticus scripsit hunc librum'. Die landwirtschaftliche Bibliothek des Benediktinerstifts Melk und ihre Leser. In: Mitteilungen des Instituts für Österreichische Geschichtsforschung 117 (2009), 3/4. S. 225-244. S. 232-237; sowie Hahn (2013), S. 437-462 (über Coler); Thirsk (1992), S. 24f.; Thirsk (1983), S. 305, 315.

${ }^{73}$ Sieglerschmidt (1999), S. 246-249.
} 
Crescentiis P de (1602) New Feldt und Ackerbau [...] von newem gemehrt worden [...] vnd durch einen Hochgelehrten deß Feldbawes wol erfahrnen/ vor Jahren in vnser Teutsche sprach an Tag gebracht. Zetzner, Straßburg.

Estienne H (1567) L'Agriculture et Maison rustique. Du-Puys, Paris.

Estienne H (1570) L'Agriculture et Maison rustique. Du-Puys, Paris.

Estienne H (1580) Siben Bücher Von dem Feldbau/ vnd vollkommener bestellung eynes ordenlichen Meyerhofs. Übs. von M Sebizius. Jobin, Straßburg.

Estienne H (1588) De Veltbouw ofte Lantwinninghe: inhoudende eene rechte wel bestellinghe eenes hofs te bouwen. Claesz, Amsterdam.

Estienne H (1592) XV. Bücher Von dem Feldbaw vnd recht volkommener Wolbestellung eines bekömmlichen Landsitzes. Übs. von M Sebizius. Jobin, Straßburg.

Estienne H (1600) Maison Rustique. Or The Covntrie Farme. Übs. von R. Surflet. Bollisant/Norton, London.

Estienne H (1606) Agricoltvra nvova, et casa di villa di Carlo Stefano. Übs. von H. Cato. Valentin, Venedig.

Estienne H (1609) Agricoltvra et casa di villa di Carlo Stefano. Übs. von H. Cato. Tarino, Turin.

Estienne H (1616) The Countrey Farme. Hrsg. und übs. von G. Markham. Bill/Islip, London.

Estienne H (1653) L'Agriculture et Maison Rvstiqve. Rigaud, Lyon.

Frühsorge G (1986) 'Georgica curiosa'. Vom geistlichen Sinn der Anleitungsliteratur bei Wolf Helmhard von Hohberg. In: H. Zeman (ed) Die österreichische Literatur. Ihr Profil von den Anfängen im Mittelalter bis ins 18. Jahrhundert (1050-1750), Teil 2. Akademische Druck- und Verlagsanstalt, Graz. S. 1071-1086.

Glacken T (1967) Traces on the Rhodian shore. Nature and culture in western thought from ancient times to the end of the eighteenth century. University of California Press, Berkeley.

Groen J, Nyland P (1670) Den Nederlandtsen Hovenier. Den Ervaren HuysHouder. Doornick, Amsterdam.

Hahn P (2013) Das Haus im Buch. Konzeption, Publikationsgeschichte und Leserschaft der "Oeconomia" Johann Colers. Bibliotheca academica, Epfendorf.

Hohberg WH v. (1701a) Georgica curiosa aucta. Bd. 1. Endter, Nürnberg. 
Hohberg WH v. (1701b) Georgica curiosa aucta. Bd. 2. Endter, Nürnberg.

McRae A (1992) Husbandry Manuals and the Language of Agrarian Improvement. In: M. Leslie, T. Raylor (ed) Culture and Cultivation in Early Modern England. Leicester UP, Leicester. S. 35-62.

Peil D (1983) Untersuchungen zur Staats- und Herrschaftsmetaphorik in literarischen Zeugnissen von der Antike bis zur Gegenwart. Fink, München.

Raylor T (1992) Samuel Hartlib and the Commonwealth of Bees. In: M. Leslie, T. Raylor (ed) Culture and Cultivation in Early Modern England. Leicester UP, Leicester. S. 91-129.

Richarz I (1991) Oikos, Haus und Haushalt. Ursprung und Geschichte der Haushaltsökonomik. Vandenhoeck \& Ruprecht, Göttingen.

Schmidt-Voges I (2008) Oíko-nomía. Wahrnehmung und Beherrschung der Umwelt im Spiegel adeliger Haushaltungslehren im 17. und 18. Jahrhundert. In: H. Düselder et al. (ed) Adel und Umwelt. Horizonte adeliger Existenz in der Frühen Neuzeit. Böhlau, Köln, Weimar, Wien.

Serres O de (1603) Le Theatre d'Agricvltvre et Mesnage des Champs [...] Seconde Edition. Saugrain, Paris.

Sieglerschmidt J (1999) Die virtuelle Landwirtschaft der Hausväterliteratur. In: R. P. Sieferle (ed) Natur-Bilder: Wahrnehmungen von Natur und Umwelt in der Geschichte. Campus, Frankfurt, New York. S. 223-254.

Thirsk J (1983) Plough and pen. Agricultural writers in the seventeenth century. In: T. H. Aston et al. (ed) Social relations and ideas: essays in honour of R. H. Hilton. Cambridge UP, Cambridge. S. 295-318.

Thirsk J (1992) Making a Fresh Start. Sixteenth-Century Agriculture and the Classical Tradition. In: M. Leslie, T. Raylor (ed) Culture and Cultivation in Early Modern England. Leicester UP, Leicester. S. 15-34.

Thomas K (1983), Man and the Natural World. Changing Attitudes in England 1500-1800. Penguin, Harmondsworth.

Tusser T (1570) Five Hundred Points of Good Husbandry. Hrsg. von G. Grigson. Oxford UP, Oxford, New York.

Wall W (1996), Renaissance National Husbandry. Gervase Markham and the Publication of England. In: Sixteenth Century Journal 27.3. S. 767-785.

Wimmer E (1998) Biene und Honig in der Bildersprache der lateinischen Kirchenschriftsteller. Österreichischer Kunst- und Kulturverlag, Wien. 
Winiwarter V (2009) 'Haereticus scripsit hunc librum'. Die landwirtschaftliche Bibliothek des Benediktinerstifts Melk und ihre Leser. In: Mitteilungen des Instituts für Österreichische Geschichtsforschung 117 (2009), 3/4. S. 225-244.

Winiwarter V, Schmidt M (2008) Umweltgeschichte als Untersuchung sozionaturaler Schauplätze? Ein Versuch, Johannes Colers „Oeconomia“ umwelthistorisch zu interpretieren. In: T. Knopf (ed) Umweltverhalten in Geschichte und Gegenwart. Attempto, Tübingen. S. 158-173.

Worlidge J (1681) Systema Agriculturae. Dring, London. 



\title{
Interactions between the Australian and German Environmental Movements
}

\author{
Astrid Mignon Kirchhof
}

In Bezug auf die australische und deutsche Umweltbewegung gibt es spannende Verflechtungen, die bisher von der geschichtswissenschaftlichen Forschung kaum thematisiert wurden. Im Zuge einer immer häufiger in den Blick geratenden transnationalen Geschichte bietet sich der umweltgeschichtliche Bereich in besonderer Weise an danach zu fragen, wie sich die Globalisierung im umweltpolitischen Bereich niederschlug und ob bzw. wie vernetzt die Bewegungen waren. ${ }^{1}$ So harren unerwartete Verbindungen zwischen Aktivisten und Gruppen in Australien und Deutschland sowie der Ideen- und Symboltransfer zwischen der Umweltbewegung beider Länder der Untersuchung. Dieser Artikel möchte einige Schlaglichter auf diese Überschneidungen werfen, die noch punktuell und teilweise unverbunden sind: Erstens wird es um die Parallelentwicklungen in den Naturschutz- und Umweltbewegungen beider Länder gehen. Zweitens werden die Verbindungen zwischen zwei Protagonistinnen, die in der Anti-Atomkraftbewegung aktiv waren, aufgezeigt, nämlich Helen Caldicott und Petra Kelly. Das Verbindende beider Akteurinnen ist, dass sie sowohl in der eigenen Bewegung - zumindest zeitweise prominent, aber auch in der Umweltbewegung des jeweils anderen Landes, vor allem als Ideengeberinnen, nicht unbedeutend waren. Drittens wird auf das Entstehen der Partei „Die Grünen“ in beiden Ländern fokussiert. Mit einer Konzentration auf die Entwicklungen in Australien zeigt der Artikel auf, dass es einerseits symbolträchtige Mythen und andererseits motivierende Ideen und Unterstützung zwischen beiden Ländern gab.

\footnotetext{
${ }^{1}$ Kirchhof AM, Meyer J-H (2014) Introduction. In: A. M. Kirchhof, J.-H. Meyer (ed) Global Protest against Nuclear Power. Transfer and Transnational Exchange in the 1970s and 1980s. HSR 39 (2014) 1, p. 165-190.
} 


\section{Introduction}

Both countries - Australia and Germany - have a tradition of strong environmental movements and the Greens. While it is well known that German environmental practices have had a decisive impact on international conservation efforts and Green movements world wide, it is not as well known that Australia was the first country to have a green party on a parliamentary level. The Australian sociologist Jan Pakulski has argued that even though there were some differences between the movements in Germany and Australia, they followed similar processes in their formation and consolidation. These included an increase in the number of protest groups, a broadening of the scope of the protests to related issues and more universal concerns, the unification of single issue protests into multi-issue events, the networking and cooperation of different groups, and the emergence of leading personalities at the national level. ${ }^{2}$

Both countries started off with single-issue campaigns and even similar topics such as hydroelectric power or the formation of national parks. In 1967 the intended flooding of Lake Pedder for energy production in the Australian state of Tasmania turned a single-issue environmental protest into a political and social movement, ${ }^{3}$ and the world's first green party, the United Tasmania Group (UTG) ${ }^{4}$, was founded. Disputes around hydroelectric power and water regulation belong to the classic conflicts in nature protection. In Germany the development of hydroelectric power was booming in the 1950s and 60s. In Bavaria alone there were protests and campaigns against the Walchenseekraftwerk, the Jochstein-Kraftwerk and the Isar-, Ammerseeand Amperregulation. ${ }^{5}$ None of these campaigns led the way to the founding of a party in Germany 6 or to a broadening in scope in both countries.

In the 1960s the "Little Desert campaign" was fought in Australia for the establishment of a national park instead of the construction of private family housing and farms on a piece of land in Victoria. Around the same time in Germany, the Verein Naturpark fought for the declaration of national parks like the Naturpark Pfälzerwald. The primary purpose of national parks is to preserve natural and cultural resources for future generations. They stand for the successful management

\footnotetext{
2 Pakulski J (1991) Social Movements. The Politics of Moral Protest. Melbourne, p. 186. For an article that shows connections between Australia and Germany in more depth, see Kirchhof AM (2014) Spanning the Globe: West-German Support for the Australian Anti-Nuclear Movement. In: A. M. Kirchhof, J.-H. Meyer (ed) Global Protest against Nuclear Power. Transfer and Transnational Exchange in the 1970s and 1980s. HSR 39 (2014) 1, p. 254-273.

${ }^{3}$ Dieter Rucht points out the definition of a social movement: "A social movement can be defined as an action system comprised of mobilised networks of individuals, groups and organisations which, based on a shared collective identity, attempt to achieve or prevent social change, predominantly by means of collective protest." See Rucht D (1999) The Transnationalization of Social Movements: Trends, Causes, Problems. In: Social Movements in a Globalizing World. Routledge, London, p. 206222. Here p. 207.

${ }^{4}$ That is the precursor of the Australian Greens.

${ }^{5}$ Engels JI (2006) Naturpolitik in der Bundesrepublik. Ideenwelt und politische Verhaltensstile in Naturschutz und Umweltbewegung 1950-1980. Schöningh, Paderborn, p. 159.

6 "Germany" refers to West-Germany in this article.
} 
of natural resources for a sustainable (economically and socially balanced) use of nature. Jens Ivo Engels holds the opinion that,

"Reine Naturschutz- oder Umweltkonflikte hat es wohl nur selten gegeben, oder besser: Erfolgreiche Widerstandsbewegungen stritten kaum ausschließlich für Vögel, seltene Pflanzen, gegen Abgase, eine Straße oder eine bestimmte Art der Energieerzeugung. [...] In jedem einzelnen Fall hatte jeder beteiligte Akteur in der Regel mehrere Motive". 7

Therefore, activists could have economic reasons or the need for information about what was going on in their area. Additionally, protest often arose to demonstrate citizens' wishes for participation in political decisions.

Particularly this last motive, the urge to shape politics, increasingly led to a broadening and networking of the environmental movements in Australia and Germany. Only after 1970 did movements become broader, more closely linked, longer in duration and often involving spectacular protests with still unaccustomed acts of civil disobedience.

Indeed, at the beginning of the 1970s, apocalyptic scenarios emanating from overseas sources such as the Club of Rome's "Limits to Growth" raised concerns about resource depletion and pollution on a global scale in both Germany and Australia. Environmental groups started to take a closer look at the needs of society. Inherent to the global aspect of the growing movements that had derived from single-issue initiatives was: participants knew that the environment did not stop at national borders.

\section{The Emergence of an Anti-nuclear Movement}

One important issue in both Germany and Australia was anti-nuclear protest. The municipality of Wyhl, near Freiburg in Baden Württemberg, with its 3,000 residents, still today has a legendary reputation in the founding mythology of the movement. The background to the founding of action groups and different campaigns was that from 1973 the state government of Baden Württemberg, together with the public energy company Badenwerk, had planned here a nuclear power plant with two reactors. ${ }^{8}$ In parallel, an alternative movement was developing in Germany. Its members tried to practice a life that was both environmentally compliant, social, and which also allowed room for individual development and space. Activists of the new social movements, together with classic nature conservation groups, founded green parties that unified to become the Greens in 1980.9

\footnotetext{
7 Engels (2006), p. 172.

8 Ebd., p. 351.

${ }^{9}$ Founding groups of the Greens were amongst others: Grüne Liste Umweltschutz, Grüne Aktion Zukunft, Aktionsgemeinschaft Unabhängiger Deutscher, Bunte Listen. See Richter S (2010) Die Aktivistin. Das Leben der Petra Kelly. Dt. Verl.-Anstalt, München, p. 177ff.; Kirchhof AM (2011) Saskia Richter: Die Aktivistin. Book review. http://hsozkult.geschichte.hu-berlin.de/rezensionen/2011-2-043. Abruf: Februar 2014.
} 
The roots of the Australian antinuclear movement go back to the debates at about the same time in 1972/73 over French nuclear testing in the Pacific and in 1976/77 over uranium mining in Australia.

One of the earliest critics of French nuclear testing was the Australian pediatrician Helen Caldicott. About the beginnings of the movement in Australia in 1972 she said a few years later:

"I came to France with an Australian delegation to speak to representatives of the French government. They said: 'Our nuclear bombs are absolutely safe.' And we asked: 'If they are so safe why don't you just test them in the Mediterranean?' And they replied: 'For god's sake, there are too many people living there!' Then we knew how dishonest they were and they knew it too. And for the first time in my life it was clear to me that I sat across from politicians who were not telling the truth, who knew that they would most likely kill people and who didn't seem at all troubled by that fact." 10

Caldicott was just as harsh in her criticism of her own government. During the 1975 oil crisis it had become clear to her that the Australian Prime Minister, Gough Whitlam, had plans to sell uranium to Europe. She perceived this business deal as not only wrong but repugnant:

"This is some kind of hypocrisy if Whitlam goes off to Europe to sell uranium on the open market just after we started mobilizing international support in protest against French nuclear testing." 11

Helen Caldicott, who had reached international fame in certain circles by way of her many book publications, media appearances and the founding of numerous alliances against the manufacturing and spread of nuclear weapons, was born in Melbourne in 1938. She was one of the founders of Physicians for Social Responsibility, an association of over 20,000 doctors and professors whose goal it was through their research to educate the public on the risks of nuclear energy and weapons. Furthermore, she took part in the founding of the organisation International Physicians for the Prevention of Nuclear War (German: Internationale Ärzte für die Verhütung des Atomkrieges, Ärzte in sozialer Verantwortung e.V.). In 1980 Caldicott retired from practicing medicine and dedicated herself full-time to her political work as an anti-nuclear activist.

One year before she made the decision to end her medical career, she travelled to Germany to attend the first women's anti-nuclear congress that took place on

\footnotetext{
${ }_{10}$ Caldicott H (1979) In jedem Atomkraftwerk lagern 1000 Hiroshima-Bomben: Bericht der australischen Ärztin Helen Caldicott. In: Courage. Berliner Frauenzeitung 4. Berlin, p. 13. In the same year 1972, Australia and New Zealand appealed to the International Court of Justice regarding France's nuclear tests on the Mururoa Atoll. The French government then announced that they would stop above ground testing, although they continued the tests underground and only ended the program in 1995.

${ }^{11}$ Ebd., p. 13.
} 
September 15 and 16, 1979 in Cologne. ${ }^{12}$ Over 100 women turned up to participate in the two-day congress. Besides Helen Caldicott, the two later founding members of the Greens, Petra Kelly and Eva Quistorp, were also present.

Various representatives of the Bundesvorstand der Bürgerinitiativen Umweltschutz (BBU), the Demokratische Fraueninitiative (DFI), the Kommunistischer Bund $(\mathrm{Kb})$, the Bunte Liste and activists of the women's movement met twice in the months before to organize the conference. At one of these organizing meetings the women delivered a petition directed against the Federal Republic's nuclear policies, including the signatures of close to 18,000 women to the Bonn Committee on Petitions. ${ }^{13}$ The task at the congress was to ensure that the voices of all women who opposed nuclear energy and weapons were heard. The first objective was to push through a federal referendum. The women also held a few spectacular political actions such as a "Die In," where passers-by in front of the Cologne Cathedral had to step over the bodies of women pretending to be dead. ${ }^{14}$ In 1979, the year of the anti-nuclear congress, Kelly left the SPD and served as the nation-wide choice for the newly founded party, the Greens, in the European Parliament elections. At the same time she became a member of the president board of the Bundesverband Bürgerinitiativen Umweltschutz (BBU) - she had already been a member of the organisation for some time. In BBU she was responsible for international contacts. ${ }^{15}$ At the anti-nuclear congress she spoke as European candidate for the Greens and presented an overview of the topic "How to move forward economically without nuclear energy." 16 This was a rather pressing subject given that the dominant view in SPD circles at the time was that without nuclear energy the current levels of prosperity in the Federal Republic would fall behind. ${ }^{17}$ At the congress Helen Caldicott addressed in her speech the danger of radioactive materials. On the importance of the speeches for the women present at the congress, the then editor of the Berlin women's magazine "Courage," Ellen Diederich, later wrote that:

"Helen Caldicott, the Australian pediatrician, the initiator of the IPPNW, doctors against nuclear energy, really shook us up. She deserves credit for taking the complex process of the entire nuclear cycle and relaying it to the audience in a language that everyone can understand. She explained to us the current international state of devel-

\footnotetext{
${ }^{12}$ Congress program 1979, Archiv Grünes Gedächtnis, Petra Kelly Archiv (AGG, PKA), 4002.

13 C M (1979) Antiatom-Kongreß. Petitionsübergabe und Kongreßvorbereitungen. In: Courage. Berliner Frauenzeitung 4, H. 8, p. 6.

${ }^{14}$ This form of protest was originally used in the US and Australia to point out the threat of atomic power. See "Vergeßt uns nicht - wir arbeiten zusammen". Die Anti-Uran-Bewegung in Australien. Petra K. Kelly im Gespräch mit Ros Livingston. In: Umweltmagazin (1979), p. 29-32. Here: p. 29. AGG, PKA, 4002.

15 The federal party "The Greens" developed in 1979 out of voter associations and electoral alliances like the "Green Lists". The party was then founded in January 1980 at the Federal Convention in Karlsruhe. See Richter (2010), p. $177 \mathrm{ff}$.

${ }^{16} \mathrm{C}$ M (1979), p. 8.

${ }^{17}$ Ebd., p. 7.
} 
opment of military and civil use of nuclear energy and informed us about the nuclear weapons already stationed in Germany."18

Helen Caldicott and Petra Kelly, one born in Australia and the other in Germany and both politically socialised in the United States, sought and established contacts outside national borders. Both women were convinced that a global network of the ecology movement was crucial. ${ }^{19}$ A contributing factor to their networking outlook was that ecology movements, and more specifically the anti-nuclear movement, were fundamentally globally oriented, even if the realisation of the movements' own convictions were constantly disputed or challenged locally. The exchange between activists from both countries was in no way one-sided, and vital impulses and exchanges came from both sides.

Besides cooperation and support between activists of both countries, the following example of the Green Bans shows how mutual references helped to strengthen the movements.

\section{The Green Ban Movement}

The background to the green-ban struggles is the story of the architectural ruin of Australia's major cities in the 1960s and early 1970s, when vast amounts of money were poured into property development: giant glass and concrete buildings changed the face of cities and valuable old buildings were razed in the process. The interests of homebuyers and architectural heritage lost out against often purely speculative construction. At one stage, there was ten million square feet of vacant office space in Sydney's business district, while people looking for their first homes or flats could find nothing. In 1971, a group of middle class women from the fashionable suburb of Hunter's Hill in Sydney, the capital of the Australian state New South Wales (NSW), teamed up with the NSW branch of the Builders Labourers' Federation (BLF), a trade union of communist oriented construction workers under the leadership of Jack Mundey. Together they saved an area of natural bushland in Sydney from destruction. This bushland was the last remaining open space in that area: the plan had been to build luxury housing on the site. This was not the only victory scored by what came to be known as the Green Bans movement. This movement prevented billions of dollars worth of undesirable development projects from being realised in the city of Sydney and rescued streets, old buildings, parks and even whole suburbs from the developer's hammer. The name green-ban was given to distinguish it from a black-ban, a union action to protect the economic

\footnotetext{
${ }^{18}$ Diederich E (2006) Petra Kelly. Mutig, zäh, subversiv und zärtlich. Visionärin für eine andere Welt. In: Lebenshaus-Newsletter (online magazine), p. 6 (eigene Zählung). http://www.lebenshaus-alb.de/magazin/003925.html. Abruf: Mai 2011.

19 Petra Kelly also published in Germany about Australian environmental topics, thus bringing them closer to the German people, see Kelly P (1979) „Lasst das Uran in der Erde!“ Die AntiUranbewegung in Australien. In: Forum Europa, No. 7/8.
} 
interests of its own members. In this case the union was going against the immediate economic interests of its members for the sake of a wider community and environmental interest. Forty-two bans were imposed between 1971 and 1974. Green bans helped to protect historic nineteenth century buildings in The Rocks ${ }^{20}$ from being demolished to make way for office towers, and prevented the Royal Botanical Garden ${ }^{21}$ from being turned into a carpark for the Sydney Opera House. ${ }^{22}$

Different authors state that when Petra Kelly was visiting Sydney in the mid1970 s at the invitation of the local anti-uranium movement, she witnessed the effectiveness of the Green Bans and was impressed with this movement, the connection between environmentalists and the Builders Labourers Federation (BLF), and the success of many of their campaigns. It is said that the green bans had a great impact on both Kelly herself and her philosophy, so that she took this concept and especially the terminology back to Germany. The word "green" supposedly was applied to the emerging environmental movement that gave rise to the German Greens. The senator and founding member of the Australian Greens, Bob Brown, proudly related this story in parliament a few years after the Australian Greens were founded in 1992. Although this story cannot be proven (Tully points out that the term "green" probably was used before the 1970s), it shows how important such references can be in political processes. ${ }^{23}$

\section{The Greens}

Unlike West Germany, the Australian Greens do not trace their beginnings back to the anti-nuclear movement but rather to a successful campaign in the 1980s against the construction of a dam on the Franklin River in Tasmania.

The dam was proposed for the purpose of generating hydroelectricity. This would have subsequently impacted upon the environmentally sensitive Franklin River, which joins the nearby Gordon River.

In 1978, the Tasmanian Hydro Electric Commission announced their intention to build the dam. The idea polarised the Tasmanian community. It gained support from some sections of the community for generating jobs in an area of the state that was struggling economically. It was suggested that the construction of the dam would assist in bringing industry to Tasmania, on top of the jobs that it would create directly. The initial opinion polls showed around $70 \%$ support for the dam.

\footnotetext{
20 That is an urban locality, tourist precinct and historic area of Sydney's city centre.

21 These are the most central of the three major botanical gardens open to the public in Sydney.

22 Burgmann V (2000) The social responsibility of labour versus the environmental impact of property capital: the australian green bans movement. In: Environmental Politics 9, No. 2, p. 78-102. The Opera House opened 1973.

23 Bevan RA (2001) Petra Kelly: The Other Green. In: New Political Science 23, No. 2, p. 181-202. See also: Burgmann M (2011) 'Green Bans movement', Dictionary of Sydney. http://www.dictionaryofsydney.org/entry/entry/green_bans_movement. Abruf: February 2014; Tully J (2004) Australia Green Bans and the BLF: the labour movement and urban ecology. In: International Viewpoint (Online magazine), IV357, footnote 8. Abruf: Februar 2014.
} 
However, the protest movement that had gathered to fight the construction of the Lake Pedder Dam earlier in the 1970s began to reassemble in response to the announcement. Under activist Bob Brown, the Tasmanian Wilderness Society, which had formed from the anti-Lake Pedder Dam groups, the Tasmanian Conservation Trust and the Australian Conservation Foundation, began to mount a public interest campaign concerning the river. ${ }^{24}$

Petra Kelly returned to Australia in 1984 and there she is said to have urged the various Green groups to develop a national identity since in Germany the Greens had formed a party four years before. Just after Christmas of that same year and partly as a result of Kelly's urging, around 50 Green activists gathered in Tasmania to organize a national conference, although it would not be until 1992 that Green representatives from around the nation would agree to form the Australian Greens. It is surprising that it took over a decade longer than in Germany for the Greens to be founded on a federal level. This is because many Australian activists were convinced that a more coherent political strategy was necessary. But most of them preferred the concept of a professionalised movement that combined mass mobilisations and campaigning with skilful lobbying, whereas a smaller group was skeptical of the difficult decisions that parties like the Australian Labour Party (ALP) would make on the environment and wanted an independent Green party. .25

Since this time the contact between both movements was not entirely dismantled. The German Greens continued to seek interaction and exchange beyond their borders and also remained in consistent contact with the Australian movement, as the following examples show.

The Australian and German movements were also in close contact in the 1980s, mostly through individual activists. The unofficial yet de facto executive director of the Australian Greens, who until this time had had no formal board, was Bob Brown. He for instance sought help from the German anti-nuclear movement by asking Petra Kelly in a letter for her support in a demonstration in front of the Australian Embassy against the destruction of the Tasmanian forest. ${ }^{26}$

Kelly was often invited to speak in Australia and, for instance, in the late 1980s travelled to the environmental summit of the Australian Conservation Foundation, a non-profit organisation founded in 1966 that was dedicated to the sustainable protection of nature and the environment as a matter of national importance in Australia, East Timor and Papua New Guinea. The organisation was also politically

24 Hutton D, Connors L (1999) A history of the Australian Environment Movement. Cambridge Univ. Press, Cambridge; Zelko F (2006) The Tasmanian Crucible: Bob Brown and the Australian Greens. In: F. Zelko, C. Brinkmann (ed) Green Parties. Reflections on the first three decades. Heinrich Böll Foundation, Washington, p. 8-20.

${ }^{25}$ Lohrey A (2007) The Rise of the Greens. In: Four Classic Quaterly Essays on Australian Politics. Melbourne, p. 165-256. Here p. 198. Also Cahill D, Brown S (2008) The Rise and Fall of the Australian Greens: the 2002 Cunningham by-election and its Implications. In: Australian Journal of Political Science 43, No. 2, p. 259-277.

26 As the SPIEGEL reported, this letter was confiscated and opened by the police, so that Kelly received the news from Brown too late, see o. N. (1989) Personalien. Petra Kelly. In: Der Spiegel 35, p. 201. 
involved in the campaign against the Franklin Dam. Its long-time president was Peter Garrett, the former singer of the Australian rock band Midnight Oil, who subsequently joined the Australian Labor Party (ALP) and was later appointed Minister for the Environment, Heritage and the Arts. This example is notable for what sets it apart from its German environmental and natural conservationist counterparts. While natural conservation organisations in Germany used to be more conservatively oriented, and a merging of both the conservation movement and the environmental movement only became feasible after 1990, it appears that natural conservation organisations in Australia had no serious problems in accepting a rock singer as their president.

\section{Conclusion}

This article represents a preliminary, explorative analysis of the connections and the transfer of ideas and symbols between the Australian and German environmental movements. A closer look at the anti-nuclear movement and the activists Helen Caldicott and Petra Kelly showed that both environmental movements dealt with similar topics and there was an exchange of ideas and reciprocal stimulation between the two sides. This exchange was predominantly on a personal level, at least during the period under examination. Even if there is no proof of terminology transfer and the importance of the Green Ban Movement as name patron for the German Greens so far, the example still illustrates the significance of mutual references. In the given example of the speech by Bob Brown, the Australian Greens aimed to demonstrate their influence by highlighting their links to the already influential German Greens. Further research is required to determine whether there was a broader basis linking the two movements. What was the significance of these links and which other topics need to be examined to be able to analyse the connections as well as the similarities and differences between both movements?

\section{Literatur}

Bevan RA (2001) Petra Kelly: The Other Green. In: New Political Science 23, No. 2, p. 181-202.

Burgmann M (2011) 'Green Bans movement', Dictionary of Sydney. http://www.dictionaryofsydney.org/entry/entry/green_bans_movement. Abruf: February 2014.

Burgmann V (2000) The social responsibility of labour versus the environ-mental impact of property capital: the australian green bans movement. In: Environmental Politics 9, No. 2, p. 78-102. 
Cahill D, Brown S (2008) The Rise and Fall of the Australian Greens: the 2002 Cunningham by-election and its Implications. In: Australian Journal of Political Science 43, No. 2, p. 259-277.

Caldicott H (1979) In jedem Atomkraftwerk lagern 1000 Hiroshima-Bomben: Bericht der australischen Ärztin Helen Caldicott. In: Courage. Berliner Frauenzeitung 4. Berlin.

C M (1979) Antiatom-Kongreß. Petitionsübergabe und Kongreßvorbereitungen. In: Courage. Berliner Frauenzeitung 4, H. 8.

Diederich E (2006) Petra Kelly. Mutig, zäh, subversiv und zärtlich. Visionärin für eine andere Welt. In: Lebenshaus-Newsletter (online magazine). Abruf: 31.08.2006.

Engels JI (2006) Naturpolitik in der Bundesrepublik. Ideenwelt und politische Verhaltensstile in Naturschutz und Umweltbewegung 1950-1980. Schöningh, Paderborn.

Hutton D, Connors L (1999) A history of the Australian Environment Movement. Cambridge Univ. Press, Cambridge.

Kelly P (1979) „Lasst das Uran in der Erde!“ Die Anti-Uranbewegung in Australien. In: Forum Europa, No. 7/8.

Kirchhof AM (2011) Saskia Richter: Die Aktivistin. Book review. http://hsozkult.geschichte.hu-berlin.de/rezensionen/2011-2-043. Abruf: Februar 2014.

Kirchhof AM (2014) Spanning the Globe: West-German Support for the Australian Anti-Nuclear Movement. In: A. M. Kirchhof, J.-H. Meyer (ed) Global Protest against Nuclear Power. Transfer and Transnational Exchange in the 1970s and 1980s. HSR 39 (2014) 1, p. 254-273.

Kirchhof AM, Meyer J-H (2014) Introduction. In: A. M. Kirchhof, J.-H. Meyer (ed) Global Protest against Nuclear Power. Transfer and Transnational Exchange in the 1970s and 1980s. HSR 39 (2014) 1, p. 165-190.

Lohrey A (2007) The Rise of the Greens. In: Four Classic Quaterly Essays on Australian Politics. Melbourne, p. 165-256.

Mez L (1979) Der Atomkonflikt. Atomindustrie u. Anti-Atom-Bewegung im internationalen Vergleich. Olle \& Wolter, Berlin.

Pakulski J (1991) Social Movements. The Politics of Moral Protest. Melbourne.

o. N. (1989) Personalien. Petra Kelly. In: Der Spiegel 35, p. 201.

Richter S (2010) Die Aktivistin. Das Leben der Petra Kelly. Dt. Verl.-Anstalt, München. 
Rucht D (1999) The Transnationalization of Social Movements: Trends, Causes, Problems. In: Social Movements in a Globalizing World. Routledge, London, p. 206-222.

Tully J (2004) Australia Green Bans and the BLF: the labour movement and urban ecology. In: International Viewpoint (Online magazine), IV357. Abruf: März 2004.

Zelko F (2006) The Tasmanian Crucible: Bob Brown and the Australian Greens. In: F. Zelko, C. Brinkmann (ed) Green Parties. Reflections on the first three decades. Heinrich Böll Foundation, Washington, p. 8-20. 



\title{
Transnationale Umweltgeschichte
}

\author{
Patrick Kupper
}

\section{Einleitung}

Die Einsicht, dass die Natur keine nationalen Grenzen kennt, verlangt in Zeiten des sich verschärfenden Klimawandels nach keinen langen Begründungen. Zugleich zeigen die zähflüssigen internationalen Verhandlungen, Treibhausgase global zu reduzieren, dass der Nationalstaat als Regulierungsmacht noch lange nicht ausgedient hat, trotz allen Geredes über dessen Erosion im Zeichen der Globalisierung. In welchem Verhältnis stehen Welt, Natur und Nation, wie hat sich dieses Verhältnis entwickelt und wie wiederum lässt sich diese Entwicklung historisch fassen, das sind die Fragen, denen ich in diesem Essay nachgehen möchte. Mein Ziel ist nicht, diese Fragen zu beantworten, sondern einen Ansatz zu entwickeln, der diese Fragen adäquat zu erfassen erlaubt und den ich als transnationale Umweltgeschichte bezeichnen möchte. Ich trage meine Argumentation in drei Schritten vor: In einem ersten Schritt werde ich in die transnationale Geschichte einführen. Sodann werde ich auf die Schnittfelder zwischen Umweltgeschichte und transnationaler Geschichte eingehen und aufzeigen, welches Potential hier vorhanden ist. Mit der transnationalen Geschichte des Nationalparks werde ich abschlieBend ein empirisches Beispiel aus meiner eigenen Forschungspraxis anführen, in dem sich der Ansatz einer transnationalen Umweltgeschichte meines Erachtens bewährt hat. 


\section{Transnational}

Transnationale Geschichte ist eine junge Erscheinung. Sie fasste zunächst in den amerikanischen Geschichtswissenschaften Fuß, wo Historikerinnen und Historiker in den 1990er Jahren ein Konzept suchten, um die überkommene Interpretation des American exceptionalism zu überwinden und die nationale Geschichtsschreibung zu öffnen. Einen zentralen Beitrag zu dieser Diskussion leistete 1999 eine Sondernummer des Journals of American History „The Nation and Beyond: Transnational Perspectives on United States History“. Im Leitartikel erklärte David Thelen das Anliegen der versammelten Beiträge wie folgt:

„Since it seemed increasingly strange that history had centered its concern with time and place on the nation-state, we wanted to design a special issue that would interrogate, not assume, the centrality of the nation-state as the organizing theme for American history. We wanted to explore how people and ideas and institutions and cultures moved above, below, through, and around, as well as within, the nation-state, to investigate how well national borders contained or explained how people experienced history. We wanted to observe how people, moving through time and space according to rhythms and relationships of their own, drew from, ignored, constructed, transformed, and defied claims of the nation-state. To make our project recognizable to others we tried, perhaps foolishly, to find a single term to encompass the many questions that sprang from our goal of reexamining history's embrace of the nation-state. We finally settled on transnational."1

Die deutschsprachige Geschichtswissenschaft nahm die transnationale Herausforderung mit einigen Jahren Verspätung auf. Die Kategorie des Transnationalen war ein Anstoß unter mehreren, mit denen eine bereits ältere Debatte um Vergleich und Transfer neu aufgemischt wurde. ${ }^{2}$ In ähnlicher Absicht wurden Beziehungsgeschichte $^{3}$, entangled history ${ }^{4}$, Histoire Croisée ${ }^{5}$ oder transkulturelle Geschichte vorgeschlagen. In der stark theoretisch-methodisch ausgerichteten Diskussion vermochte sich in der Folge keiner der Vorschläge entscheidend vom Feld abzu-

\footnotetext{
1 Thelen (1999), S. 967. Weitere wichtige Beiträge sind Bender T (ed) (2001) Rethinking American History in a Global Age. University of California Press, Berkeley; Bender T (2006) A nation among nations. America's place in world history. Hill and Wang, New York.

2 Conrad S u. Osterhammel J (2004) Das Kaiserreich transnational. Deutschland in der Welt 18711914. Vandenhoeck \& Ruprecht, Göttingen. Zur älteren Debatte siehe Kaelble H (ed) (2003) Vergleich und Transfer: Komparatistik in den Sozial-, Geschichts- und Kulturwissenschaften. Campus, Frankfurt a. M.

${ }^{3}$ Osterhammel J (2001) Geschichtswissenschaft jenseits des Nationalstaats: Studien zur Beziehungsgeschichte und Zivilisationsvergleich. Vandenhoeck \& Ruprecht, Göttingen.

${ }_{4}^{4}$ Conrad S u. Randeria S (ed) (2002) Jenseits des Eurozentrismus. Postkoloniale Perspektiven in den Geschichts- und Kulturwissenschaften. Campus, Frankfurt a. M.

5 Werner M, Zimmermann B (2006) Beyond Comparison. Histoire Croisée and the Challenge of Reflexivity. In: History and Theory $(45,1)$. S. 30-50.

${ }^{6}$ Herren M (2012) Transkulturelle Geschichte. Globale Kultur gegen die Dämonen des Eurozentrismus und des methodischen Nationalismus. In: Traverse (1, 2). S. 154-169.
} 
setzen. ${ }^{7}$ Inzwischen sind diese Auseinandersetzungen in den Hintergrund getreten. Sie haben zwar keine einheitliche Lösung gebracht, aber zum einen die Gemeinsamkeiten klar gemacht, die all diese Ansätze verbindet, insbesondere die zentrale Absicht, raumzeitliche Ordnungen historiographisch zu erschließen und ihre geschichtsprägenden Einflüsse sichtbar zu machen. Zum anderen schärfte die Diskussion die Aufmerksamkeit gegenüber methodischen Herausforderungen, die mit einem solchen Unterfangen verbunden sind. Insofern trug die Theoriediskussion wohl dazu bei, dass die empirische Forschung, die in den letzten Jahren verstärkt eingesetzt hat, sowohl auf einer soliden theoretisch-methodischen Basis operieren kann als auch die verwandten Ansätze mit einer wohltuenden Abgeklärtheit zu nutzen und zu kombinieren vermag. So hat sich das Themenportal Europäische Geschichte, das sich in dieser Richtung besonders hervorgetan hat, die Aufgabe gestellt, Beiträge zu sammeln, welche die Geschichte Europas seit dem 18. Jahrhundert als Problemgeschichte angehen: „vergleichend, transfer- und verflechtungsgeschichtlich und im Spannungsfeld zwischen Nationalisierung und Globalisierung. " 8

Allerdings wäre es fatal, den freimütigen Umgang mit theoretischen Angeboten mit einer beliebigen Kombinierbarkeit zu verwechseln. Die Wahl von Begrifflichkeiten und Konzepten muss forschungspraktische Konsequenzen haben, ansonsten verlieren diese Instrumente ihr Distinktionspotential und damit auch ihre Erklärungskraft. Daher macht eine Entscheidung für das Konzept des Transnationalen meines Erachtens nur dann Sinn, wenn die Nation der zentrale Referenzpunkt der historischen Analyse ist. Der Sinn des Attributs transnational besteht gerade darin, die nationale Dimension in der Geschichtsschreibung zwar zu relativieren, ohne die Nation aber als erklärungsmächtige historische Kategorie aufzugeben. ${ }^{9}$ Wie Thelen in seinen oben zitierten Ausführungen plastisch darstellt, dient das Präfix trans dazu, der vielfältigen Gestalt jener Prozesse Ausdruck zu geben, welche nationale Räume transzendieren und damit zugleich deren Grenzen perforieren. Die enge Verschränkung mit der Kategorie des Nationalen spricht dafür, die Verwendung des Konzepts zeitlich und räumlich einzugrenzen: Die transnationale Geschichte ist für die Geschichte der modernen Welt zuständig, deren Konturen sich seit dem Ende des 18. Jahrhunderts auszuprägen beginnen, um sich Mitte des 19. Jahrhunderts zu verfestigen. Für frühere Zeiten und andere Räume gibt es passendere Ansätze. ${ }^{10}$

\footnotetext{
7 Das 2004 gegründete Webforum geschichte.transnational (http://geschichte-transnational.clioonline.net) etablierte sich rasch als zentrale Kommunikationsplattform. Siehe die Rubrik Forum. Zudem: Budde G, Conrad S, Janz O (ed) (2006) Transnationale Geschichte. Themen, Tendenzen und Theorien. Vandenhoeck \& Ruprecht, Göttingen.

${ }^{8} \mathrm{http}: / /$ www.europa.clio-online.de/site/lang_de-DE/40208575/Default.aspx. Abruf: 23.12.2012.

9 Zur Diskussion um den Zuständigkeitsbereich transnationaler Geschichte siehe Gassert P (2012) Transnationale Geschichte. Docupedia-Zeitgeschichte. Version: 2.0. Abruf: 29.10.2012.

10 Auf den Zeitraum seit 1850 beschränkt sich etwa der Palgrave dictionary of transnational history Iriye A, Saunier PY (ed) (2009) The Palgrave Dictionary of Transnational History. Palgrave Macmillan, Basingstoke. Zum Konzept der modernen Welt siehe Bayly CA (2004) The Birth of the Modern World. 1780-1914. Global Connections and Comparisons. Blackwell, Malden.
} 
Da die Nation eine europäische Erfindung ist, verbindet sich mit der transnationalen Geschichte zudem zwangsläufig eine eurozentrische Sichtweise der Geschichte. Dafür kann sie kritisiert werden. Dagegen ist zu halten, dass zum einen die Perspektivierung transparent ist und zum anderen die Zentralstellung Europas eine Rechtfertigung in dessen im Untersuchungszeitraum global dominanten Einfluss findet. Eines der herausragenden Kennzeichen dieses Zeitabschnitts war letztlich die Globalisierung des nationalstaatlichen Modells, das mit dem Niedergang der Imperien zur weltweit dominanten politischen Verfassung aufstieg. ${ }^{11}$ Dies ist in der Globalisierungsdebatte der letzten beiden Jahrzehnte weitgehend übersehen worden. Globalisierung und Nationalstaat wurden als antagonistische Kräfte diskutiert, und erstere als Totengräberin nationalstaatlicher Ordnungen porträtiert. Der unweigerliche Bedeutungsverlust des Nationalstaats wurde wortreich beklagt, manchmal, jedoch weit seltener, auch begrüßt. Dagegen haben Sebastian Conrad und andere eingewendet, dass sich in einer zeitlich ausgedehnteren Perspektive zeigt, dass sich Globalisierung und Nationalstaat gegenseitig bedingten und befruchteten. ${ }^{12}$ Ich würde einen Schritt weitergehen und die globale Etablierung nationalstaatlicher Standards und die nationalstaatliche Befestigung globaler Unterschiede als ein hervorragendes Kennzeichen dieser stark durch Europa und die USA geprägten Epoche bezeichnen.

Transnationale Geschichte führt notwendig sowohl über die Nation als auch über Europa hinaus. Daraus ergeben sich Verwandtschaften zu einer Reihe von Forschungsfeldern, insbesondere zu jenen der postkolonialen und neuen imperialen Geschichte, deren Verflechtungsgeschichten ausgeprägte Überschneidungen mit dem transnationalen Untersuchungsfeld aufweisen. ${ }^{13}$ Gegenüber den teils in ähnlicher (teils aber auch in anderer) Absicht verwendeten Bezeichnungen Global-, Welt- oder Universalgeschichte hat die Verwendung des Attributs transnational den Vorteil, nicht wie jene die Erwartung zu wecken, gleich alle Zeiten und Orte zu berücksichtigen. Dies ist nur in den seltensten Fällen sinnvoll und in noch weniger Fällen befriedigend zu leisten. ${ }^{14}$ Schließlich ist eine Unterscheidung zum Beg-

\footnotetext{
11 Siehe etwa Schulze H (1994) Staat und Nation in der europäischen Geschichte. Beck, München; Anderson B (1991) Imagined Communities. Reflections on the Origin and Spread of Nationalism. Verso, London. Zum Verhältnis von Nationalstaat und Imperium: Leonhard J, Hirschhausen U v. (2009) Empires und Nationalstaaten im 19. Jahrhundert. Vandenhoeck \& Ruprecht, Göttingen.

12 Conrad S (2006) Globalisierung und Nation im deutschen Kaiserreich. Beck, München.

13 Siehe etwa Conrad u. Randeria (2002); Cooper F (2005) Colonialism in Question. Theory, Knowledge, History. University of Califiornia Press, Berkeley; Chakrabarty D (2007) Provincializing Europe. Postcolonial thought and historical difference. Princeton University Press, Princeton; Howe S (ed) (2010) The new imperial histories reader. Routledge, London.

${ }_{14} \mathrm{Zu}$ Global- und Weltgeschichte siehe Manning P (2003) Navigating World History. Historians Create a Global Past. Palgrave Macmillan, New York; Conrad S, Eckert A, Freitag U (ed) (2007) Globalgeschichte. Theorien, Ansätze, Themen. Campus, Frankfurt a. M.; Osterhammel J (ed) (2008) Weltgeschichte. Steiner, Stuttgart. Für eine Universalgeschichte traditionellen Zuschnitts: Weber WEJ (2001) Universalgeschichte. In: M. Maurer (ed) Aufriss der Historischen Wissenschaften, Band 2. Räume. Reclam, Stuttgart. S. 15-98. Zu den unterschiedlichen Forschungstraditionen, die sich hinter den Begriffen verstecken, siehe Middell M (2005) Universalgeschichte, Weltgeschichte, Globalgeschichte, Geschichte der Globalisierung. Ein Streit um Worte? In: M. Grandner et al. (ed) Globalisierung und Globalgeschichte. Mandelbaum, Wien. S. 60-82.
} 
riff international angebracht: Dieser sollte den Beziehungen auf offiziöser staatlicher Ebene vorbehalten bleiben, während unter dem Begriff transnational unterschiedliche, die nationalen Räume überschreitende Bewegungen materieller und diskursiver Art zu subsumieren sind.

\section{Umweltgeschichte}

Der Bezugsrahmen der transnationalen Geschichte ist die europäisch geprägte Welt der Moderne. Deren Entstehung und Entwicklung ist von Christopher Bayly und Jürgen Osterhammel in meisterhaften Synthesen dargestellt worden, welche der weiteren Forschung wohl für geraume Zeit einen Orientierungsrahmen geben. ${ }^{15}$ Insbesondere Jürgen Osterhammel räumt der Umweltgeschichte in seiner Darstellung breiten Raum ein, wobei er zugleich deren mangelnden Forschungsstand beklagt. ${ }^{16}$ Osterhammels Hinwendung zu umweltgeschichtlichen Fragestellungen scheint sich aus seiner globalgeschichtlichen Perspektive und seinem im weiten Sinn transnationalen Zugriff ergeben zu haben. Tatsächlich kann und sollte die Umweltgeschichte einen zentralen Platz in der Erschliessung der Geschichte der modernen Welt beanspruchen. Natur ist eine zentrale Grösse dieser Geschichte. Sie tritt in mannigfacher Weise auf: als Widerstand und Ressource, als originäre Referenz und Gegenstand gesellschaftlicher Zurichtung, etc. Die Umweltgeschichte ist daher herausgefordert, ihre Kompetenzen einzubringen. Zugleich eröffnet sich der Umweltgeschichte die grosse Chance, ihre Forschungsinteressen und -kompetenzen in den Geschichtswissenschaften breiter zu verankern, eine Chance, die sie sich nicht entgehen lassen sollte; insbesondere im deutschsprachigen Raum, wo Umweltgeschichte weiterhin ein institutionelles Nischendasein fristet. So ist es bezeichnend, dass in den USA Umwelthistoriker wie Richard White und Ian Tyrell die transnationale Diskussion massgebend mitgeprägt haben. In der nachgelagerten deutschen Diskussion sucht man vergebens nach vergleichbaren Interventionen.

Eine transnationale umwelthistorische Herangehensweise, wie ich sie hier propagieren möchte, darf sich nicht auf eine räumliche Ebene fixieren. Sie ist herausgefordert, verschiedene räumliche Ebenen in die Analyse einzubeziehen, diese untereinander zu verknüpfen und zu untersuchen, wie räumliche Arrangements menschliches Denken und Handeln steuerten und wie Denken und Handeln umgekehrt räumliche Arrangements hervorbrachten. Hierzu muss die räumliche Skala, auf der die Analyse angesetzt wird, variiert werden. Komplexität kann nicht reduziert werden, indem kleinere Strukturen in größeren aufgelöst werden, wie dies die Weltgeschichtsschreibung, aber auch die Nationalgeschichte in ihren synthetisierenden Darstellungen traditionell betrieben haben. Noch lässt sich Geschichte in

\footnotetext{
15 Bayly (2004); Osterhammel J (2009) Die Verwandlung der Welt. Eine Geschichte des 19. Jahrhunderts. Beck, München.

${ }^{16}$ Ebd., S. 541.
} 
einem einmal definierten Raum festmachen und verhandeln. Diese raumzeitliche Problematik wurde bereits in den 1990er Jahren, angeregt durch die italienische Mikro-Historie, in den Kreisen der Historischen Anthropologie verhandelt. Der Historiker Jacques Revel sprach von einem Jeux d'échelles, einem Spiel mit Grössenordnungen. ${ }^{17}$ Diese Texte lesen sich auch heute noch mit Gewinn, ebenso wie der Beitrag Richard Whites zur eingangs genannten Sondernummer, in der er ein umwelthistorisches Konzept multipler Räume vorschlägt. ${ }^{18}$

Schließlich darf der transnationale Zusammenhang nicht à priori gesetzt werden. An Stelle des Nationalen das Transnationale zu naturalisieren, wäre eine verhängnisvolle Entwicklung. Transnationale Kontexte dürfen in anderen Worten nicht vorausgesetzt werden, vielmehr sind sie in der empirischen Forschungsarbeit herauszupräparieren, in konkreten, beobachtbaren und beschreibbaren Verknüpfungen historisch zu etablieren. Die Kategorien des „Lokalen“, „Nationalen“ und „Globalen“ sind historisch nicht stabil, sondern müssen von den historischen Akteuren fortlaufend erneuert und zueinander in Beziehung gesetzt werden. Dabei können sie sowohl aufgebrochen als auch stabilisiert werden. Vermehrte Beachtung verdient dabei die Ausbildung von Überlappungszonen, die zwischen Räumen unterschiedlicher Verfasstheit entstehen und über längere Zeit bestehen können, ohne dass sich die Differenzen in ihnen aufheben würden. Einen inspirierenden Ansatz hat ebenfalls Richard White mit dem Konzept des middle ground geliefert. ${ }^{19}$

Wer sich auf eine transnationale Herangehensweise einlässt, sieht sich einigen forschungspraktischen Schwierigkeiten gegenüber. Zum einen lässt sich im Ver-

\footnotetext{
${ }^{17}$ Revel J (ed) (1996) Jeux d'échelles. La micro-analyse à l'expérience. Gallimard, Paris. Zur MikroHistorie siehe Levi G (1992) On Microhistory. In: P. Burke (ed) New Perspectives on Historical Writing. Pennsylvania State University Press, University Park. S. 97-119; Ginzburg C (1993) MikroHistorie. Zwei oder drei Dinge, die ich von ihr weiss. In: Historische Anthropologie (2). S. 169-192. Wichtige Inspirationsquelle war Sigfried Kracauers posthum erschienenes Buch (Kracauer S (1971) Geschichte - Vor den letzten Dingen. Suhrkamp, Frankfurt a. M.) in dem er für einen permanenten Wechsel der Aufnahmegrössen, von „close-ups“ und „long shots“, eintritt. Einen kompetenten Einstieg in diese Diskussionen bietet Tanner J (2004) Historische Anthropologie zur Einführung. Junius, Hamburg. S. 101-118. Vorbild können jene Arbeiten sein, die sich in systematischer Art darum bemühen, globale und lokale Entwicklungen empirisch zu verknüpfen. Etwa Bayly (2004), Cooper (2005), Hopkins AG (ed) (2006) Global History. Interactions between the Universal and the Local. Palgrave Macmillan, New York, Conrad (2006). Die wechselseitige Konstituierung lokaler und globaler Räume wird auch unter dem Begriff Glokalisierung verhandelt: Robertson R (1995) Glocalization. Time-Space and Homogeneity-Heterogeneity. In: M. Featherstone et al. (ed) Global Modernities. Sage, London. S. 25-44.

18 White R (1999) The Nationalization of Nature. In: Journal of American History (86, 3). S. 976-986. Vgl. auch Tyrrell I (2001) Beyond the View from Euro-America. Environment, Settler Societies, and the Internationalization of American History. In: T. Bender (ed) Rethinking American history in a global age. University of California Press, Berkeley. S. 168-191; Taylor JE III. (2008) Boundary Terminology. In: Environmental History (13, July). S. 454-81; Evans S (2010) Recent Developments in Transnational Environmental History. Labor, Settler Communities, and Comparative Histories. In: Radical History Review (107). S. 195-208.

19 White R (1991) The middle ground. Indians, empires, and republics in the Great Lakes region. 1650-1815. Cambridge University Press, Cambridge. Whites Geschichte spielt sich in einem vortransnationalen Setting ab, das Konzept ist aber auf transnationale Untersuchungen übertragbar.
} 
gleich zu Projekten nationalen Zuschnitts der Rahmen sehr viel schlechter abstecken, den eine Untersuchung möglicherweise annehmen wird. Der Forschungsprozess ist weniger kalkulierbar, was auch befürchten lässt, dass entsprechende Anträge es bei der tendenziell risikoscheuen Forschungsförderung schwer haben könnten. Zum anderen, aber auch damit zusammenhängend, stellen die historischen Überlieferungen neue Herausforderungen, da gerade die klassischen staatlichen Archivbestände überwiegend national organisiert sind. Transnationalen Spuren zu folgen kann sich daher rasch als sehr aufwändig erweisen. Streng methodisch begründete Suchparameter sind oft wenig zielführend, während eine heuristische Suche, die nach plausiblen Verknüpfungen forscht, ergiebigere Resultate zeigen dürfte. Auf jeden Fall öffnet sich innovativen Historikerinnen und Historikern ein weites Praxisfeld. Den neuen Bedürfnissen angemessene Forschungsstrategien $\mathrm{zu}$ entwickeln und entsprechende Forschungszusammenhänge zu etablieren, ist eine der vordringlichen Aufgaben der kommenden Jahre.

\section{Transnationale Nationalparks}

Abschließend möchte ich einen Einblick in meine eigene Forschungspraxis in transnationaler Umweltgeschichte geben. Da die Ergebnisse inzwischen publiziert sind, kann ich mich kurz halten und auf die entsprechenden Verröffentlichungen verweisen, insbesondere meine Monographie Wildnis schaffen sowie den Sammelband Civilizing Nature. ${ }^{20}$ In diesen Arbeiten versuchte ich zusammen mit Fachkolleginnen und -kollegen, die globale Geschichte des Nationalparks in transnationaler Perspektive neu zu interpretieren. Entgegen gängiger Erzählungen ist die Entwicklung der Nationalparkidee und die globale Verbreitung von Nationalparks weder als simple Reaktion auf Naturzerstörung noch als von den USA ausgehende, lineare Diffusion zu begreifen. Vielmehr zeigt sich in der transnationalen Rekonstruktion, dass die Beziehung zwischen Naturzerstörungen und gesellschaftlichen Wahrnehmungen und Handlungen komplex waren. Die weltweite Verbreitung von Nationalparks und entsprechendem Ideengut und Praktiken folgte keinem festen Schema, sondern vollzog sich in einem Spiel von Übernahme und Aneignung, von Oktroyierung und Rückweisung, von Anlehnung und Abgrenzung. So wurde der Begriff Nationalpark zwar tatsächlich erstmals für den 1872 in den USA geschaffenen Yellowstone National Park verwendet. Weiterreichende Bedeutung gewann der Begriff aber erst an der Wende zum 20. Jahrhundert, als transnational eine rasch wachsende Naturschutzbewegung den weltweiten Verlust von Naturräumen und biologischen Arten zu thematisieren begann und den Schutz grosser zusammenhängender Flächen als Gegenmassnahme propagierte. Erst jetzt fand die Nati-

\footnotetext{
${ }^{20}$ Kupper P (2012) Wildnis schaffen. Eine transnationale Geschichte des Schweizerischen Nationalparks. Haupt, Bern; Gissibl B, Höhler S, Kupper P (ed) (2012) Civilizing Nature. National Parks in Global Historical Perspective. Berghahn, New York.
} 
onalparkidee Anhänger auf allen Kontinenten und erst in diesem Zusammenhang wurde Yellowstone zum transnational diskutierten Schutzmodell. ${ }^{21}$

Transnationale Geschichte behandelt die moderne Welt. Der Nationalpark ist eine geradezu archetypische Einrichtung dieser transnationalen Moderne. Begriffe wie Zivilisierung und Entwicklung, Territorialisierung und Kategorisierung sind für das Verständnis der Moderne zentral und sie sind es ebenso für die Nationalparkgeschichte. So muss die Ausscheidung von Gebieten als Nationalparks als Teil jenes umfassenden Territorialiserungsprozesses gesehen werden, den der amerikanische Historiker Charles Maier als den zentralen Prozess erachtet, der die globale Entwicklung seit ca. 1860 steuerte und vorantrieb. ${ }^{22}$ Ebenso schrieb sich die Geschichte des Nationalparks in das System dualer Kategorien ein, das Bruno Latour als ein Kennzeichen der Moderne herausgearbeitet hat. ${ }^{23}$ Der Nationalpark wurde als ein Raum der Natur oder Wildnis konzipiert, der vom gesellschaftlichen Raum, dem Raum der Kultur oder Zivilisation, abzutrennen war. Er verkörperte das „Andere“, den sakralen Raum einer säkularisierten und zivilisierten Moderne. In dieser Dichotomie erblickte etwa der Präsident der Forschungskommission des Schweizerischen Nationalparks, der renommierte Botaniker Carl Schröter, die Bedeutung des Nationalparks:

„Er [der Nationalpark] ist in erster Linie eine Stätte, wo jegliche Einwirkung des Menschen für alle Zeiten ausgeschaltet ist, wo alpine Urnatur sich ungestört wiederherstellen und weiterentwickeln kann und wird. Er bildet ein Refugium für Pflanzen- und Tierwelt, ein Sanktuarium, ein Naturheiligtum. An seinen Grenzen brechen sich die über alle Lande strömenden Wogen menschlicher Kultur, die das ursprüngliche Antlitz der Mutter Erde zerstören: er ist aus der, Ökumene', aus der Besiedlungssphäre ausgeschaltet.“24

Als geschichtslose Wildnis oder urzeitliche Natur war der Nationalpark dem Einfluss des Menschen entzogen, blieb ihm aber dennoch zugänglich. Er war der modernen Zivilisation zugleich Enklave und Spiegel: In ihm ließ sich die Illusion pflegen, Natur nicht nur frei von menschlichem Einfluss in ihrem „ursprünglichen“ Zustand zu erhalten, sondern auch anhand der Differenz zum gesellschaftlichen

${ }^{21}$ Diese Interpretation wird in den USA weiterdiskutiert. Siehe Tyrrell I (2012) America's National Parks. The Transnational Creation of National Space in the Progressive Era. In: Journal of American Studies $(46,1)$. S. 1-21 und die dortigen Diskussionsbeiträge von Astrid Swenson, Paul S. Sutter und Thomas R. Dunlap. Zudem das Projekt National Parks Beyond the Nation (http://nationalparksbeyondthenation.wordpress.com). Abruf: 23.12.2012.

22 Maier CS (2000) Consigning the Twentieth Century to History. Alternative Narratives for the Modern Era. In: American Historical Review $(105,3)$. S. 807-831. Maier sieht die Prägekraft der Territorialität seit 1970 am Schwinden. Für eine kritische Diskussion dieser These siehe Gissibl B, Höhler S, Kupper P (2012) Towards a Global History of National Parks. In: B. Gissibl et al. (ed) Civilizing Nature. National Parks in Global Historical Perspective. Berghahn, New York. S. 1-27. Hier S. 11-13.

${ }^{23}$ Latour B (1995) Wir sind nie modern gewesen. Versuch einer symmetrischen Anthropologie. Akademie Verlag, Berlin.

${ }^{24}$ Schröter C (1918) Der schweizerische Nationalpark im Unterengadin. In: Die Naturwissenschaften (6, 52). S. 761-765. Hier S. 763. 
Raum die kulturellen Errungenschaften bemessen und bewerten zu können. Darüber hinaus ließ sich die Errichtung einer solchen Institution als eigene zivilisatorische Errungenschaft feiern. In der säuberlichen Trennung in Kultur und Natur, Zivilisation und Wildnis offenbart sich der Nationalpark nicht nur als von der Moderne durchdrungenes Konzept, sondern auch als aktiver Träger und Gestalter der dialektischen Ordnung der Moderne. Seine Geschichte bietet einen privilegierten Einblick in den Wandel des gesellschaftlichen Umgangs mit Natur seit dem ausgehenden 19. Jahrhundert sowie in die Globalisierung des Nationalen und die Nationalisierung des Globalen.

\section{Literatur}

Anderson B (1991) Imagined Communities. Reflections on the Origin and Spread of Nationalism. Verso, London.

Bayly CA (2004) The Birth of the Modern World. 1780-1914. Global Connections and Comparisons. Blackwell, Malden.

Bender T (ed) (2001) Rethinking American History in a Global Age. University of California Press, Berkeley.

Bender T (2006) A nation among nations. America's place in world history. Hill and Wang, New York.

Budde G, Conrad S, Janz O (ed) (2006) Transnationale Geschichte. Themen, Tendenzen und Theorien. Vandenhoeck \& Ruprecht, Göttingen.

Chakrabarty D (2007) Provincializing Europe. Postcolonial thought and historical difference. Princeton University Press, Princeton.

Conrad S (2006) Globalisierung und Nation im deutschen Kaiserreich. Beck, München.

Conrad S, Eckert A, Freitag U (ed) (2007) Globalgeschichte. Theorien, Ansätze, Themen. Campus, Frankfurt a. M.

Conrad S, Osterhammel J (2004) Das Kaiserreich transnational. Deutschland in der Welt 1871-1914. Vandenhoeck \& Ruprecht, Göttingen.

Conrad S, Randeria S (ed) (2002) Jenseits des Eurozentrismus. Postkoloniale Perspektiven in den Geschichts- und Kulturwissenschaften. Campus, Frankfurt a. M.

Cooper F (2005) Colonialism in Question. Theory, Knowledge, History. University of Califiornia Press, Berkeley. 
Evans S (2010) Recent Developments in Transnational Environmental History. Labor, Settler Communities, and Comparative Histories. In: Radical History Review (107). S. 195-208.

Gassert P (2012) Transnationale Geschichte. Docupedia-Zeitgeschichte. Version: 2.0. Abruf: 29.10.2012.

Ginzburg C (1993) Mikro-Historie. Zwei oder drei Dinge, die ich von ihr weiss. In: Historische Anthropologie (2). S. 169-192.

Gissibl B, Höhler S, Kupper P (ed) (2012) Civilizing Nature. National Parks in Global Historical Perspective. Berghahn, New York.

Gissibl B, Höhler S, Kupper P (2012) Towards a Global History of National Parks. In: B. Gissibl et al. (ed) Civilizing Nature. National Parks in Global Historical Perspective. Berghahn, New York. S. 1-27.

Herren M (2012) Transkulturelle Geschichte. Globale Kultur gegen die Dämonen des Eurozentrismus und des methodischen Nationalismus. In: Traverse $(1,2)$. S. 154-169.

Hopkins AG (ed) (2006) Global History. Interactions between the Universal and the Local. Palgrave Macmillan, New York.

Howe S (ed) (2010) The new imperial histories reader. Routledge, London.

Iriye A, Saunier PY (ed) (2009) The Palgrave Dictionary of Transnational History. Palgrave Macmillan, Basingstoke.

Kaelble H (ed) (2003) Vergleich und Transfer: Komparatistik in den Sozial-, Geschichts- und Kulturwissenschaften. Campus, Frankfurt a. M.

Kracauer S (1971) Geschichte -- Vor den letzten Dingen. Suhrkamp, Frankfurt a. M.

Kupper P (2012) Wildnis schaffen. Eine transnationale Geschichte des Schweizerischen Nationalparks. Haupt, Bern.

Latour B (1995) Wir sind nie modern gewesen. Versuch einer symmetrischen Anthropologie. Akademie Verlag, Berlin.

Leonhard J, Hirschhausen U v. (2009) Empires und Nationalstaaten im 19. Jahrhundert. Vandenhoeck \& Ruprecht, Göttingen.

Levi G (1992) On Microhistory. In: P. Burke (ed) New Perspectives on Historical Writing. Pennsylvania State University Press, University Park. S. 97-119.

Maier CS (2000) Consigning the Twentieth Century to History. Alternative Narratives for the Modern Era. In: American Historical Review (105, 3). S. 807-831. 
Manning P (2003) Navigating World History. Historians Create a Global Past. Palgrave Macmillan, New York.

Middell M (2005) Universalgeschichte, Weltgeschichte, Globalgeschichte, Geschichte der Globalisierung. Ein Streit um Worte? In: M. Grandner et al. (ed) Globalisierung und Globalgeschichte. Mandelbaum, Wien. S. 60-82.

Osterhammel J (2001) Geschichtswissenschaft jenseits des Nationalstaats: Studien zur Beziehungsgeschichte und Zivilisationsvergleich. Vandenhoeck \& Ruprecht, Göttingen.

Osterhammel J (ed) (2008) Weltgeschichte. Steiner, Stuttgart.

Osterhammel J (2009) Die Verwandlung der Welt. Eine Geschichte des 19. Jahrhunderts. Beck, München.

Revel J (ed) (1996) Jeux d'échelles. La micro-analyse à l'expérience. Gallimard, Paris.

Robertson R (1995) Glocalization. Time-Space and Homogeneity-Heterogeneity. In: M. Featherstone et al. (ed) Global Modernities. Sage, London. S. 25-44.

Schröter C (1918) Der schweizerische Nationalpark im Unterengadin. In: Die Naturwissenschaften $(6,52)$. S. 761-765.

Schulze H (1994) Staat und Nation in der europäischen Geschichte. Beck, München.

Tanner J (2004) Historische Anthropologie zur Einführung. Junius, Hamburg.

Taylor JE III. (2008) Boundary Terminology. In: Environmental History (13, July). S. 454-81.

Tyrrell I (2001) Beyond the View from Euro-America. Environment, Settler Societies, and the Internationalization of American History. In: T. Bender (ed) Rethinking American history in a global age. University of California Press, Berkeley. S. 168-191.

Tyrrell I (2012) America's National Parks. The Transnational Creation of National Space in the Progressive Era. In: Journal of American Studies (46, 1). S. 1-21.

Weber WEJ (2001) Universalgeschichte. In: M. Maurer (ed) Aufriss der Historischen Wissenschaften, Band 2. Räume. Reclam, Stuttgart. S. 15-98.

Werner M, Zimmermann B (2006) Beyond Comparison. Histoire Croisée and the Challenge of Reflexivity. In: History and Theory (45, 1). S. 30-50.

White R (1991) The middle ground. Indians, empires, and republics in the Great Lakes region. 1650-1815. Cambridge University Press, Cambridge. 
White R (1999) The Nationalization of Nature. In: Journal of American History (86, 3). S. 976-986. 


\title{
Naturgeschichte Wattenmeer
}

\author{
Karsten Reise
}

\section{Warum Naturgeschichte}

Natur hat Geschichte. Seit gut 13 Milliarden Jahren dehnt sich das Universum aus, ohne je inne zu halten. Auf unserem Planeten entsteht seit 3,8 Milliarden Jahren fortlaufend neues Leben und altes stirbt aus, besonders durch extreme Ereignisse wie globale Vereisungen und Einschläge großer Meteoriten. So verschwanden vor 65 Millionen Jahren Dinosaurier, danach lebten wir Säugetiere auf und ein Zurück wird es nicht geben. Tierische Menschen entstanden vor 6 Millionen Jahren und vor 40.000 Jahren wanderte der Homo sapiens in Europa ein. Unsere Vorfahren durchstreiften schon die Nordseeregion, bevor dort vor 8.000 bis 6.000 Jahren eine Küste mit einer langen Kette von Düneninseln und ausgedehnten Sand- und Schlickwatten aus dem Meer wuchs. Damit begann die späte Naturgeschichte des Wattenmeers.

Nicht nur die Natur hat ihre Geschichte, sondern auch was wir unter Naturgeschichte verstehen. Aristoteles erfasste damit die mit den Sinnen wahrnehmbare Welt und unterschied sie von der abstrakten Ideenwelt des Sokrates und Platon. Im 1. Jahrhundert verfasste Plinius der Ältere seine Naturalis Historia, eine naturwissenschaftliche Weltbeschreibung, in der unter anderem erstmals die seltsame Region des Wattenmeers beschrieben wurde.

Aus dieser alles umgreifenden Naturgeschichte emanzipierten sich in moderner Zeit die naturwissenschaftlichen Disziplinen. Zuletzt bezog sich die Naturgeschichte nur noch auf Mineralien, Pflanzen und Tiere. In Frankreich beschrieb in dieser Endzeit der Naturgeschichte der Comte de Buffon die Entwicklung der 
Erde und zahlreiche Pflanzen- und Tierarten in seiner vielbändigen Histoire naturelle. Zeitgleich (beide 1707 geboren) systematisierte in Schweden Carl von Linné in minimalistischem Stil fast 6.000 Arten. Den Pariser Konkurrenten ehrte er durch Benennung der runzeligen Erdkröte mit Bufo bufo. Sein Systema Naturae wurde zur Grundlage aller Artbeschreibungen, inzwischen 1,3 Millionen und etwa 10.000 davon kommen im Wattenmeer vor. ${ }^{1}$

Eine Verzeitlichung der Naturgeschichte klingt u. a. bei Emanuel Kant an, der in der Kritik der Urteilskraft 1790 über historische Wirkungsgesetze in der Natur schreibt und für möglich hält, dass „gewisse Wassertiere sich nach und nach zu Sumpftieren, und aus diesen zu Landtieren ausbildeten". ${ }^{2}$ Als Charles Darwin 1851 mit der natürlichen Selektion von zufällig entstehenden Varianten den Evolutionsprozess beschrieb und damit die Schöpfungsgeschichten ablöste, gab es die Natural History als Forschungsdisziplin schon nicht mehr, obwohl sie für Darwins Forschungen ein passender Begriff gewesen wäre. Der löste sich im Behauptungskampf der neuen naturwissenschaftlichen Disziplinen auf und Sammler und Beobachter von Naturalien wurden zu Amateuren degradiert. Der umfassende Begriff der Naturgeschichte überlebte nur in den Namen von Museen und Vereinen, die sich der Vielfalt in der Natur widmeten.

Während die Ökosystemforschung nach allgemeinen Systemeigenschaften und zeitlosen Rückkopplungsmechanismen sucht, gibt es auch einen Zweig der Ökologie, der Lebensgemeinschaften und ganze Naturräume in ihrer zeitlichen Entwicklung untersucht. Was hat sich wann und wo und warum verändert? Dabei geht es nicht um prinzipielle Vorgänge in der Natur, sondern um konkrete Ereignisfolgen und darauf passt nun wieder der alte Begriff der Naturgeschichte in seinem wörtlichen Sinn als einer Geschichte der Natur. Die wiederum wird zur Umweltgeschichte, wo sie mit gesellschaftlicher Geschichte verschmilzt. ${ }^{3}$ Im Wattenmeer war der Mensch von Beginn an dabei und teilte schließlich diesen Küstenraum in eine Kulturlandschaft, geschützt hinter hohen Deichen, und eine Naturlandschaft vor diesen Deichen, wo das Meer die breite Übergangszone zum Land in ständiger Bewegung hält. Dieser amphibische Bereich wurde 2009 von der UNESCO zum Weltnaturerbe erklärt.

\footnotetext{
${ }^{1}$ Reise K, Baptist M, Burbridge P et al. (2010) The Wadden Sea - A universally outstanding tidal wetland. Wadden Sea Ecosystem 29. Common Wadden Sea Secretariat, Wilhelmshaven.

${ }^{2}$ Lepenies W (1976) Das Ende der Naturgeschichte. Hanser Verlag, München, Wien.

${ }^{3}$ Herrmann B (2009) Umweltgeschichte Wozu? Zur gesellschaftlichen Relevanz einer jungen Disziplin. In: P. Masius, O. Sparenberg, J. Sprenger (ed) Umweltgeschichte und Umweltzukunft. Universitätsverlag Göttingen, Göttingen. S. 13-50.
} 




Abb. 1: Naturlandschaft im südlichen Dithmarschen: Watten und Priele bei Niedrigwasser, vorne entstehende Dünen auf der Vogelinsel Trischen und Dieksander Koog am Horizont (Juli 2012).

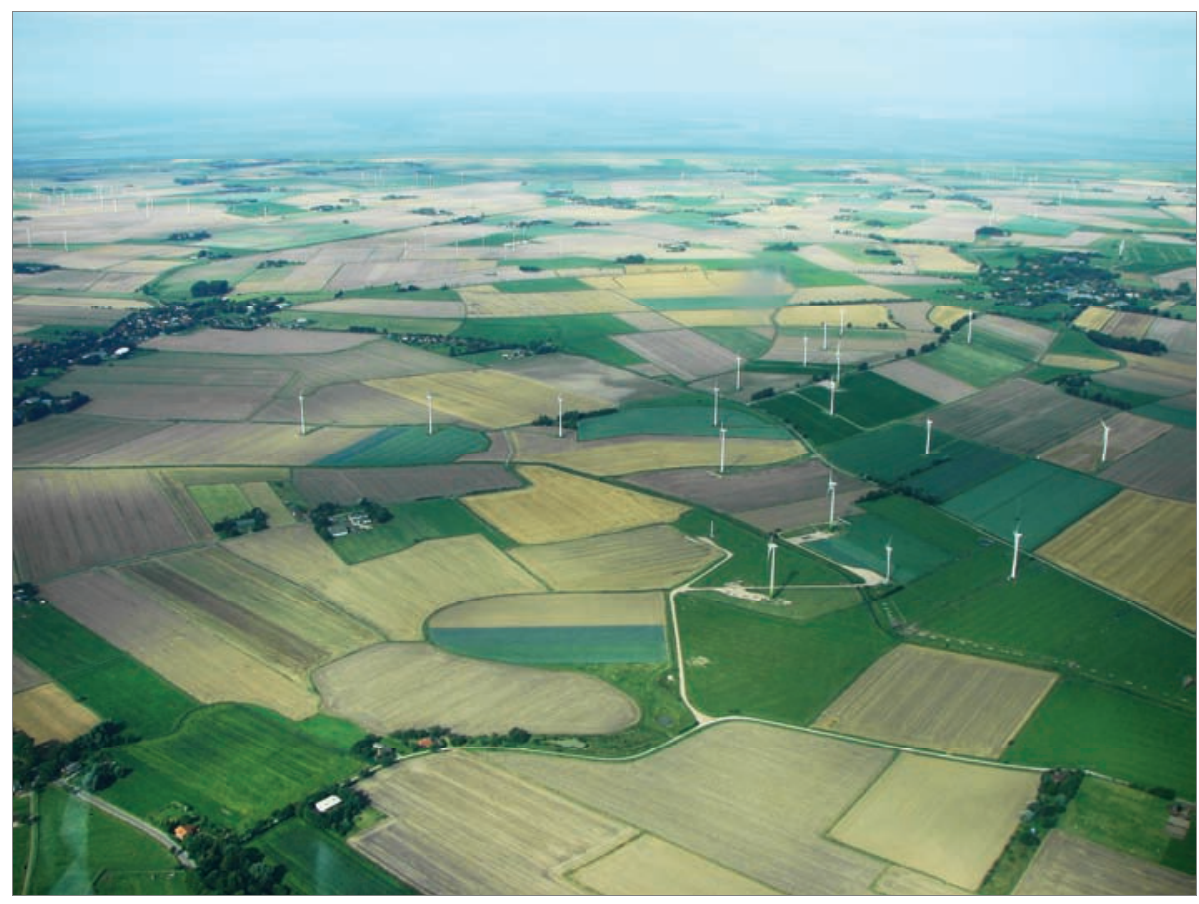

Abb. 2: Kulturlandschaft im südlichen Dithmarschen: Felder und Siedlungen in 1787 und 1854 eingedeichten Kögen (August 2009). 
Wie kann eine veränderliche und von Menschen viel besuchte und genutzte Küstenlandschaft mit ihren Naturwerten den kommenden Generationen vererbt werden? Dieser Beitrag versucht zu zeigen, dass dafür die Rückbesinnung auf die Naturgeschichte geeignetes Orientierungswissen liefern kann. Da sich die geschichtliche Betrachtung ökologischer Systeme bisher wenig etabliert hat, werde ich vorweg auf die Bedeutung der Kontingenz für Erklärungen in der Naturgeschichte eingehen, denn Zufälle haben ganz wesentlich die Entwicklung des Wattenmeers beeinflusst. Das wird an Beispielen erläutert. Verabschiedet wird damit der verbreitete Traum von einem natürlichen Gleichgewicht, dessen Ursprung in die Zeit zurückreicht, als die Naturgeschichte noch aus Geschichten über die Natur und nicht als Zeitgeschichte verstanden wurde. Dieser Anachronismus blockiert bei der Suche nach neuen Strategien, um das Wattenmeer mit seinen Naturwerten in einer sich schnell verändernden Welt erhalten zu können.

\section{Kontingenz in der Naturgeschichte}

Naturereignisse können auf verschiedene Weisen erklärt werden. Für gewöhnlich werden generelle Kausalzusammenhänge ermittelt, um zu erklären, wie die Natur funktioniert und um so bei ähnlichen Ereignissen Vorhersagen treffen zu können. Eine andere Erklärungsform sucht nicht nach dem Prinzipiellen, sondern versucht das Zustandekommen des Einzelfalls nachzuvollziehen. Dabei ist die Kontingenz ein zentraler Begriff. ${ }^{4}$ Kontingenz steht für das zufällige Zusammentreffen von Ereignissen, für Eventualität und Möglichkeit und beinhaltet eine gewisse Unsicherheit. In der Sprache der Logik sind nach Niklas Luhmann kontingente Ereignisse weder zwangsläufig noch unmöglich. Die Natur kann nicht allein als ein notwendiger Zusammenhang von Ursachen und Wirkungen verstanden werden, sondern den realen Zufällen und Wahrscheinlichkeiten ist ebenso Bedeutung beizumessen.

Kontingente Ereignisse erfordern eine spezielle Form der Erklärung. Für evolutionäre Ereignisse formuliert Gould ${ }^{5}$ dies so: Das Ereignis E trat ein, weil vorher $\mathrm{D}$ eingetreten war, dem wiederum die Ereignisse C, B und A vorhergingen. Sonst wäre es nicht zu E gekommen, sondern zu etwas anderem. Solch eine geschichtliche Erklärung wird nicht direkt von allgemeinen Gesetzmäßigkeiten der Natur abgeleitet, sondern aus der konkreten Folge von Ereignissen. Jede größere Veränderung bei auch nur einem Schritt der Ereignisfolge hätte zu einem anderen, vorläufigen Endergebnis geführt.

Ähnlich wie mit solch zeitlicher Kontingenz verhält es sich mit räumlicher Kontingenz. Das Ereignis E stellte sich an bestimmter Stelle ein, weil daneben die räumliche Domäne von $\mathrm{D}$ liegt und daneben wiederum die Domänen von $\mathrm{C}, \mathrm{B}$

\footnotetext{
${ }^{4}$ Hampe M (2011) Tunguska oder das Ende der Natur. Carl Hanser Verlag, München.

5 Gould SJ (1989) Wonderful life. The Burgess shale and the nature of history. Hutchinson Radius, London.
} 
und $\mathrm{A}$, vorausgesetzt das Auftreten von $\mathrm{E}$ erfolgt nicht unabhängig von seinen Nachbarn. Es ist weder zwingend noch ausgeschlossen, dass sich $\mathrm{E}$ an bestimmter Stelle ereignet, aber wahrscheinlich.

Kontingenz begrenzt die Reichweite von gefundenen Gesetz- und RegelmäBigkeiten. Das bedeutet nicht, dass allgemeine Gesetze, regelhafte und wiederkehrende Muster nicht auch wichtig sind, aber sie können oft von einzelnen Ereignissen mit langfristigen und weitreichenden Folgen überlagert werden. Ehe eine bekannte Abfolge zu ihrem uns absehbar erscheinenden Ende führt, wird sie durch zufällige Ereignisse abgelenkt oder vorzeitig beendet. In der Naturgeschichte erhält solche Kontingenz volle Aufmerksamkeit und wird nicht aus Respekt vor der Gesetzmäßigkeit als Geräusch im Datensatz, als Ausreißer unter Messwerten, als Kuriosität oder als Störung abgetan.

Nachfolgend zwei Beispiele für Kontingenz: Im Wattenmeer ging der verheerenden Sturmflut vom Jahre 1362 eine Pestepidemie voraus. Die Bevölkerung konnte daher die Deiche nicht ausreichend in Stand halten. Wir haben dafür das Sprichwort: Ein Unglück kommt selten allein. Das Unglück von 1362 lässt sich nicht allein mit Klima, Meeresspiegelanstieg oder Bahnen von Tiefdruckgebieten im Nordatlantik erklären und auch nicht allein mit epidemiologischen und sozialen Prozessen. Es war das unglückliche Zusammentreffen von zwei unabhängigen Ereignisfolgen, das zum Tod vieler Küstenmenschen führte.

Wenn sich im Wattenmeer Krebslarven auf geeignetem Substrat in festwachsende Seepocken verwandeln wollen, dann entscheiden sie sich für eine Anheftungsstelle nach Form und Beschaffenheit der Substratoberfläche, deren Lage im Gezeitenbereich und nach der Anwesenheit anderer Seepocken.

Unebenheiten werden bevorzugt und auch die Nähe zu anderen Seepocken, denn sonst kann später keine Vermehrung erfolgen. Außerdem sind die Überlebenschancen dicht beieinander besser als für vereinzelte Seepocken, gleich welcher Art. Selten aber ist es so, dass zu allen geeigneten Plätzen auch Larven finden und mitunter entscheiden Larven auch falsch, wenn sie nicht alle Faktoren ihrer Umgebung richtig erkennen. Verteilungsmuster von Organismen sind das Produkt allgemeiner Prinzipien in Kombination mit kontingenten Konstellationen. 




\begin{abstract}
Abb. 3: Seepocken im Gesicht einer Wachsfigur im Wasser des Büsumer Hafens. Larven dieser Krebstiere sondieren die Oberfläche, heften sich an geeigneter Stelle fest und bauen ein Gehäuse aus Kalkplatten. Runde Kegel: die einheimische Art Semibalanus balanoides; sternförmige Kegel: die aus Australien eingeschleppte Austrominius modestus (August 2011).
\end{abstract}

Die Naturgeschichte befasst sich mit solchen Einzelfällen in Zeit und Raum und fügt diese zu übergreifenden Geschichten zusammen. Da erklärt wird, wann, wo und warum etwas stattfand, befasst sich diese Naturgeschichte mit realen Gegebenheiten und nicht dem, was nach erkannten Prozessen hätte geschehen sollen. Beide Erklärungsformen, die aus einer Funktionsanalyse hervorgehende Kausalerklärung allgemeiner Art und die aus einer Kontingenzbetrachtung resultierende Erklärung des Einzelfalls, können sich je nach Fragestellung gut ergänzen. Generelle Ursache-Wirkungsketten reichen fast nie aus, um ein konkretes Muster zu erklären, weil früher oder später Kontingenzphänomene hinzutreten. In der naturwissenschaftlichen Forschungspraxis wird aber die kausale Erklärungsform deutlich favorisiert und dominiert in den Fachzeitschriften, während Einzelfallanalysen nur selten zur Publikation kommen. Auf dem Weg zu den einfachen und eleganten Erklärungen, die zu einem Universalverständnis führen könnten, werden sie als störend empfunden.

In der Naturgeschichte wird dagegen die Realgeschichte rekonstruiert, wo die Einzelfälle zählen und das nicht vorhersehbare Zusammentreffen von zuvor unabhängigen Ereignisfolgen und Zufällen volle Beachtung findet. Während abstrakte Modelle darstellen, was am wahrscheinlichsten gewesen wäre oder künftig sein 
könnte, wenn die Natur sich ohne Abweichungen an allgemeine Funktionsprinzipien gehalten hätte oder künftig halten wird, widmet sich die Naturgeschichte gerade diesen Abweichungen. Die Naturgeschichte des Wattenmeers ist reich an Koinzidenz und liefert ein gutes Beispiel für die Rolle des Zufälligen im Werdegang eines Landschaftsraumes.

\section{Die Entstehung der Wattenküste}

In der kältesten Phase der letzten Eiszeit, vor 20.000 bis 18.000 Jahren, lag so viel gefrorenes Wasser in Gletschern und Eisschilden fest auf dem Land, dass der Meeresspiegel um 120 Meter tiefer lag als heute. Wo heute die Nordsee wogt, lag eine weite Landebene so groß wie die norddeutschen Küstenländer zusammen mit Dänemark und den Niederlanden. In Tundra und Wäldern, an Flüssen und Seen lebten Mammut, Wollnashorn, Pferd, Bison, Auerochse, Elch, Rentiere, Biber, Bär und Wolf und unter ihnen jagten und fischten Menschen. ${ }^{6}$

Nach der Eiszeit stieg der Meeresspiegel ziemlich schnell um insgesamt einhundert Meter in 10.000 Jahren an.

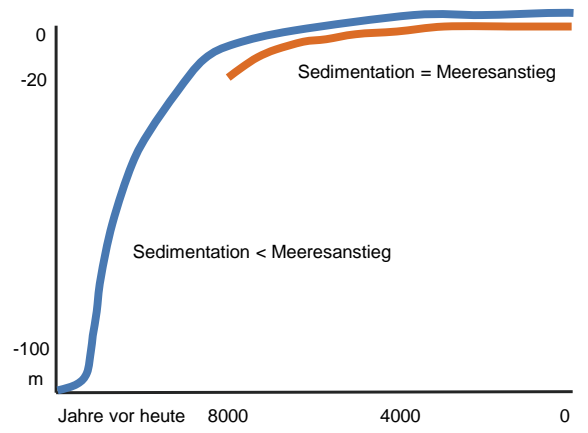

Abb. 4: Ungefährer Verlauf des Meeresanstiegs seit der letzten Eiszeit. Erst vor 8.000 Jahren konnte der Sedimenteintrag vom Meer den abklingenden Anstieg des Meeresspiegels ausgleichen und so ein Wattenmeer entstehen.

Das flache Land im südlichen Nordseebecken geriet unter Wasser. Erst entstand der Kanal zwischen England und dem Kontinent und zuletzt versank die Doggerbank. Über die Fauna wissen wir aus Knochenfunden in den Bodennetzen der Fischer. Auch Jagdwerkzeuge damaliger Menschen waren dabei. Im Schnitt stieg in

${ }^{6}$ Gaffney V, Fitch S, Smith D (2009) Europe's lost world. The discovery of Doggerland. Council for British Archaeology, York. 
dieser Zeit der Meeresspiegel um rund einen Meter in hundert Jahren. Das ist eine Größenordnung, wie sie im Zuge der globalen Klimaerwärmung in Zukunft wieder erwartet wird. Als dann aber vor etwa 8.000 Jahren die großen Eisschilde von Kanada und Skandinavien weitgehend abgetaut und deren Schmelzwasser ins Meer geflossen war, stieg das Meer nur noch langsam weiter, etwa um 15 Zentimeter in hundert Jahren. Erst jetzt konnte ein Wattenmeer entstehen. Auch anderswo auf der Welt bildeten sich seit Abflauen des Meeresanstiegs Schwemmlandschaften an den Küsten. Die großen Deltas von Nil, Mississippi oder Ganges-Brahmaputra begannen ins Meer zu wachsen, gespeist von den Sedimenten, die diese großen Flüsse mit sich führten.

Allein die Entschleunigung des nacheiszeitlichen Meeresspiegelanstiegs erklärt jedoch noch nicht die Entstehung des Wattenmeers. Entscheidend war das Zusammentreffen mit weiteren Bedingungen: (1) Die flache Nordsee wurde ein sehr bewegtes Meer mit starkem Seegang im Winter, verursacht von aus Westen kommenden Tiefdruckgebieten des Nordatlantiks. Wo die Wellen den Grund berührten, transportierten sie Sand zur Küste. Bei Sturmfluten konnte sich das Wasser um mehr als drei Meter vor der Küste aufstauen. Das verursachte bei dem flachen Terrain weit ins Land reichende Überschwemmungen. Die trugen Sedimente vom Meer aufs Land, das so mit dem Meeresanstieg mithalten konnte. (2) Zusätzlich verstärkte sich der Tidenhub mit dem Meeresanstieg. Bleibt dieser unter einem Meter, dann entstehen unter dem Einfluss von Strömungen und Wind an sandigen Küsten lange Nehrungshaken, wie wir sie heute an der amerikanischen Atlantikküste, in der südlichen Ostsee sowie westlich und nördlich des Wattenmeers vorfinden. Dahinter liegen Lagunen, die mitunter ganz verlanden. Bei höherem Tidenhub mit stärkeren Gezeitenströmungen zerfallen die sandigen Nehrungen in Inselketten und Sandbänke. Davon ist das Wattenmeer geprägt. Nimmt der Tidenhub weiter zu, werden in der Tendenz die Inseln kürzer und ab $3 \mathrm{~m}$ Tidenhub sind die Wasserbewegungen zu stark für dauerhafte Inseln. Das ist im zentralen Bereich des Wattenmeers zwischen Jade und Eiderstedt der Fall. Diese Zunahme des Tidenhubs ergibt sich aus der Buchtenform des Wattenmeers mit den Trichtermündungen der großen Flüsse, insbesondere Elbe und Weser, in der Mitte. (3) Weiterhin trug das gute Sedimentangebot zur Entstehung des Wattenmeers bei. Das Nordseebecken wurde in den vorhergehenden Millionen von Jahren durch mächtige Sandablagerungen aufgefüllt. Wellen und Gezeitenströmungen mobilisieren diesen Sand, so dass vor der Küste Sandbänke, Düneninseln und Watten entstehen konnten. Watten und Salzwiesen wachsen außerdem durch feine Schwebstoffe, die sich im Nordseeplankton und in Flussmündungen bilden.

Das Wattenmeer verdankt seine Entstehung dieser einzigartigen Konstellation. Der nur noch langsame Anstieg des Meeres konnte mehr oder weniger durch Sedimentumlagerungen in einem breiten Küstenstreifen kompensiert werden. Dadurch fand nur noch abgeschwächt eine Verschiebung des Küstenverlaufs statt. Zwischen See und Land entstand als eine Art von Puffer das Wattenmeer mit einer nur in der Mitte unterbrochenen Kette von Inseln, weiten Watten und ausgedehn- 
ten Überschwemmungswiesen. Watt und Salzwiesen wuchsen mit dem langsam weiter steigenden Meeresspiegel mit und die Inseln wanderten.

Das darf man sich nun allerdings nicht als ein natürliches Gleichgewicht vorstellen, das ein sich selbst erhaltendes Wattenmeer hervorgebracht hat. Dagegen spricht der Blick ins Detail.7 Im Westfriesischen und Nordfriesischen Wattenmeer verschob sich die äußere Küstenlinie aus Sandbänken und Düneninseln um etwa $10 \mathrm{~km}$ von Nord nach Süd bzw. von West nach Ost, während zwischen Jade und Eider das weit vorgedrungene Meer anschließend so viel Sediment ablagerte, dass dort das Land wieder ins Meer wachsen konnte. Das dänische Wattenmeer erhielt so viel Sediment von Norden her, dass es sich seewärts ausdehnte. Um ein allgemeines Erhaltungsgesetz des Wattenmeers zu konstruieren, fehlt es der Entwicklung an innerer Konsistenz. Die Entstehung des Wattenmeers ist ein kontingentes Ereignis.

Geologische Bohrungen im Watt hinter den Ostfriesischen Inseln zeigten auBerdem ein Mehrfaches Hin und Her zwischen Land und Meer. ${ }^{8}$ In Bohrkernen von $20 \mathrm{~m}$ Tiefe wechseln sich dreimal Torflagen mit Meeressedimenten ab. Behre deutete dies mit einem Auf und Ab des Meeresspiegels im Verlauf der Jahrtausende, aber dafür fehlt es an zeitlicher Konsistenz entlang der Nordseeküste. Solche Torflagen entstanden nicht gleichzeitig. Wahrscheinlicher ist daher ein kleinräumiger Wechsel zwischen Überkompensation durch starken Sedimenteintrag und einem Sedimentdefizit relativ zum Meeresspiegelanstieg sowie Zwischenzeiten, wo sich beide Prozesse die Waage hielten, während der Meeresspiegel kontinuierlich stieg.

Aus heutiger Sicht betrachtet, ist das Wattenmeer ein gelungener Zufall. Es ist das Resultat des Zusammentreffens mehrerer, voneinander nicht abhängiger Vorgänge und Voraussetzungen, die in der südlichen Nordsee vor 8.000 Jahren eine Entwicklung einleiteten, die anschließend zeitlich und räumlich einen wechselnden Verlauf nahm. Nur bei oberflächlicher Betrachtung ergibt sich das Bild einer Küste im Gleichgewicht mit den Naturkräften. Genannt wurden hier nur Meeresspiegelanstieg und Sedimenteintrag durch Wellen und Gezeitenströme. Ebenfalls von Bedeutung sind das Klima, das sich seit den letzten 11.000 Jahren nur noch wenig veränderte, außergewöhnliche Stürme und Regenfälle, ein langsam sinkendes Land und schließlich Veränderungen in Vegetation und Fauna einschließlich der Besiedlung durch den Menschen.

\section{Das größte Wattenmeer}

Anzunehmen ist, dass sich die Jäger und Fischer aus der weiten Ebene im südlichen Nordseebecken, die durch das Abschmelzen der Eisschilde nach und nach im Meer versank, an die englische Küste und in die Region des heutigen Wattenmeers

\footnotetext{
7 Oost AP, Hoekstra P, Wiersma A et al. (2012) Barrier island management: Lessons from the past and directions for the future. Ocean \& Coastal Management 68. S. 18-38.

8 Behre KE (2008) Landschaftsgeschichte Norddeutschlands. Wacholtz Verlag, Neumünster.
} 
zurückzogen. Wie auch anderswo in Europa und Asien wirkten diese Menschen mit oder waren die Ursache am Aussterben von Mammut, Wollnashorn, Steppenwisent, Auerochse oder Riesenhirsch und hatten damit indirekt Einfluss auf die Verteilung zwischen Wald und offenem Grasland. ${ }^{9}$ Eine direkte Veränderung der Küstenlandschaft begann vor 6.000 Jahren mit Siedlern der Jungsteinzeit, die Haustiere hielten und Feldfrüchte anbauten. Auf den Anhöhen, die Gletscher der vorletzten Eiszeit in der Küstenebene hinterließen, wurden Spuren dieser Siedler gefunden. Der Zahl der Grabstätten nach zu urteilen, wie z.B. auf Sylt, könnten sie dort recht dicht und lange gelebt und dabei den Baumbewuchs entfernt haben. ${ }^{10}$

Die dauerhafte Nutzung der tiefer gelegenen, fruchtbaren Marschgebiete hat entlang der erhöhten Flussbänke und auf Uferwällen vor 5.000 Jahren begonnen. Ab 2.800 vor heute wurden vermehrt künstlich erhöhte Wohnplätze angelegt, um Sturmfluten im Trocknen aussitzen zu können. ${ }^{11}$ Nach der Zeitenwende entstanden daraus regelrechte Wohnhügellandschaften in der Marsch, von denen auch Plinius der Ältere in seiner Naturalis historia berichtet hat. Diese Tjerpen, Wurten oder Warften genannten Wohnhügel wurden fortlaufend erhöht, entsprechend den erlittenen Sturmfluthöhen. Die Beweidung der umliegenden Salzwiesen war intensiv, während höher gelegene, moorige Bereiche des Hinterlands kaum genutzt wurden.

Zwischen 1000 und 1500 jetziger Zeitrechnung vollzog sich ein gravierender Wandel in der Küstenlandschaft. Anfängliche Ringdeiche, um Felder vor Überflutungen zu schützen, wurden erhöht und miteinander verbunden, so dass ab 1300 eine weitgehend geschlossene Deichlinie Überflutungen von der Nordsee und von den Flüssen her abwehrte. Das hatte weitreichende Folgen: (1) Bei Sturmfluten stand den Wassermassen weniger Überflutungsraum zur Verfügung und die Wellenenergie prallte unmittelbar gegen die Deiche. Je mehr Marsch eingedeicht wurde, desto wehrhafter mussten die Deiche werden. (2) Durch die Deiche war die Sedimentzufuhr vom Meer unterbrochen und die eingeschlossene Marsch konnte nicht mehr mit dem steigenden Meer mitwachsen. (3) In der eingedeichten Marsch wurde das Regenwasser über ein Grabensystem gesammelt und dann bei Tideniedrigwasser durch Siele im Deich abgelassen. Dies veränderte die Marschböden und führte zu Sackungen, so dass das Landniveau unter das des Meeres geriet. (4) Stellenweise wurde Torf abgebaut, was das Land noch mehr absenkte. Das geschah auch vor den Deichen, denn dort hatte Meerwasser in den fossilen Torflagen Salz hinterlassen. Dieser Torf wurde abgetragen, getrocknet und verbrannt, um mit dem gewonnenen Salz einen lukrativen Handel zu treiben. Land wurde so regelrecht abgetragen.

\footnotetext{
9 Bunzel-Drüke M, Drüke J, Vierhaus H (1994) Quaternary Park. Überlegungen zu Wald, Mensch und Megafauna. ABUinfo 17/18. S. 1-35.

10 Bantelmann A (1992) Landschaft und Besiedlung Nordfrieslands in vorgeschichtlicher Zeit. Stiftung Nordfriesland, Föhr.

${ }^{11}$ Bazelmann et al. (2012); Behre (2008); Meier (2006).
} 
Weite Bereiche gerieten unter den Meeresspiegel. Aus der eingedeichten Marsch musste das sich sammelnde Regenwasser hochgepumpt werden, meist unter Verwendung von Windkraft. Gelang dies nicht in ausreichender Weise, wurde Malaria zur Plage, weil sich in den mit Regenwasser gefüllten Gräben die Mücken gut vermehren konnten. Das schwächte die Bevölkerung und die Instandhaltung der Deiche wurde dadurch schwieriger. Da Deiche die Überflutungen abwehrten, wurden die Wohnhügel nicht weiter erhöht und neue Häuser flach auf das Land gebaut. Der Deich war nun allein für die Sicherheit zuständig, es wurde quasi alles auf eine Karte gesetzt.

Die Deiche verbesserten zwar die landwirtschaftlichen Nutzungsmöglichkeiten und die Siedlungsdichte nahm zu, aber hielt der seeseitige Deich einer Sturmflut nicht stand, waren die Folgen katastrophal. Im Laufe der Zeit war durch Eindeichung, Bodennutzung und Siedlungsform eine sehr verwundbare Küstenlandschaft entstanden. Nur wenn die Seedeiche fortlaufend verstärkt wurden, konnte diese Kulturlandschaft Bestand haben. Das war den Siedlern bewusst, aber wenn Pestepidemien, Malaria und andere Krankheiten, politischer Streit und Kriege hereinbrachen, war die „Deichlast“ nicht mehr zu tragen. Immer wieder brachen bei schweren Sturmfluten die Deiche und danach floss das Wasser aus dem abgesenkten Land nicht mehr raus. So entstanden nach und nach tief ins Land reichende Wattbuchten: Zuiderzee, Middelzee, Lauwerszee, Dollard, Leybucht, Harlebucht, Jadebusen und die Nordfriesischen Watten. Die Folge war, dass um 1500 die Wattflächen ihre maximale Ausdehnung erreichten.

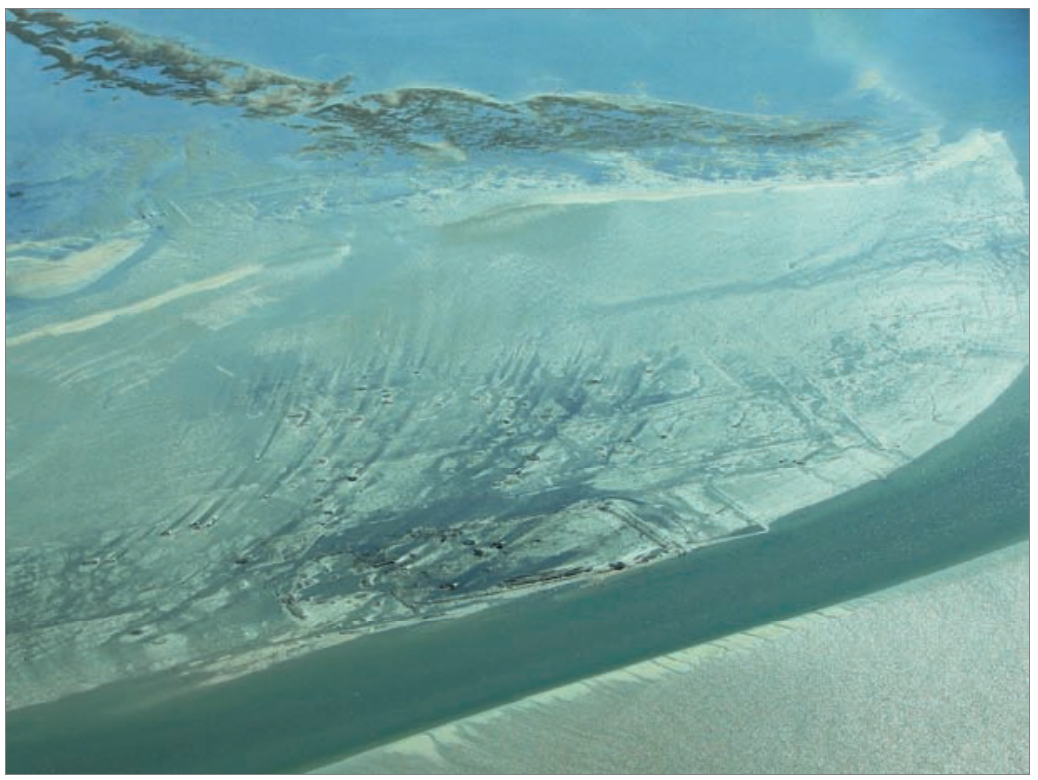

Abb. 5: Lange unter Wattsand verborgene Reste der 1634 untergegangenen Siedlung Buphever werden am Rande eines Priels freigespült. Im Hintergrund auftauchende dunkle Flecken sind Muschelbänke, Nordfriesisches Wattenmeer (Juli 2012). 
Die Ursache dafür waren nicht die Sturmfluten an sich, sondern die Verwundbarkeit der vom Menschen geschaffenen Küstenlandschaft und die Störanfälligkeit der gesellschaftlichen Strukturen. Das kontingente Zusammentreffen dieser drei Komponenten führte zu einer Folge schwerster Katastrophen, in denen Menschen ihr Leben verloren oder wegen Eigentumsverlust die Küstenregion verlassen mussten. Das biberhafte Anlegen von Wällen, die das Wasser fernhalten sollten, führte ungewollt zum Gegenteil. Ein natürlicher Küstenzustand stellte sich dadurch aber nicht wieder ein. Durch die verbliebenen oder wieder hergestellten Deiche bestand die künstlich geschaffene, abrupte Grenze zwischen Meer und Land fort. Der allmähliche Übergang vom Watt über eine breite Zone an Salz- und Brackwasserwiesen hin zu Mooren oder bewaldetem Land war nirgendwo mehr zu finden. Ebenso wie die Geschichte der Menschen nicht rückspulbar ist, so gilt das auch für die Landschaft an der Nordseeküste.

\section{Beginn einer neuen Wattallianz}

Nach den katastrophalen Sturmfluten des ausgehenden Mittelalters und der frühen Neuzeit kam es nicht zu einer strategischen Umkehr in der Gestaltung der Küstenlandschaft. Der Schrecken saß tief und in der Wahrnehmung wurde aus der Nordsee eine „Mordsee“. Doch verbesserte Deichbautechniken und großräumigere politische Strukturen ermöglichten einen immer besseren Schutz vor Sturmfluten. Verlorenes Land wurde durch künstliche Landgewinnungsverfahren zu großen Teilen zurückgewonnen. Geflochtene Buschzäune, die für Wasser durchlässig waren, aber Strömung und Wellenbewegung verringerten, vermehrten die Sedimentablagerungen vor den Deichen. Zusätzlich wurden Grabensysteme entwickelt, die nach einer Überflutung das Wasser besser abführten, so dass kein Restwasser im gewonnenen Deichvorland zurück blieb (siehe auch Abb. 8). So entstand anstelle einer mit Tümpeln und fein verzweigten Prielen durchdrungenen natürlichen Salzwiese ein geometrisch angelegtes Deichvorland, oft mit kurz gefressener Vegetation durch Schafe oder Rinder. War das Vorland hinreichend aufgewachsen, wurde es eingedeicht. Das Wattenmeer wurde so wieder kleiner.

Küstenschutz wurde zu einer gesamtstaatlichen Aufgabe. Im 20. Jahrhundert war die Deichbautechnik soweit entwickelt, dass auch lange Dämme durch das Watt gebaut wurden, um so ganze Buchten in Kulturland zu verwandeln. Dadurch wurde auch Watt eingedeicht, wo vorher nie Siedlungsland war. Am spektakulärsten war die Abdämmung der Zuiderzee (1932), die teils in Land und teils in einen Süßwassersee (Ijsselmeer) umgewandelt wurde. Die meisten Buchten wurden eingedeicht und zu den Inseln Sylt und Rømø wurden Dämme zur besseren Verkehrsanbindung gebaut (1927 und 1948). Pläne entstanden, auch die Kette von Düneninseln miteinander zu verbinden, um aus dem dahinter liegenden Watt neues Kulturland zu schaffen. Erneute Sturmflutdesaster in den Niederlanden (1953) und Deutschland (Hamburg 1962) führten zur massiven Verstärkung aller Deiche 
und vor Flussmündungen wurden Sperrwerke gegen Sturmfluten errichtet, um sich weiter landeinwärts höhere Flussdeiche zu sparen. Die strukturelle Konfrontation zwischen Meer und Land nahm dadurch zu.



Abb. 6: Konfrontation zwischen Land und Meer: Massiver Deich, davor im Watt mit Steinbuhnen, um Sedimentabtrag zu verringern, am Deichfuß Steinschüttungen, die einer Unterspülung vorbeugen. Das mittlere Tidehochwasser reicht bis an den ersten Steinabsatz, die raue Steinpackung oberhalb soll Wellenenergie brechen. Der asphaltierte Weg ist für Baufahrzeuge, Abtransport von Treibsel und Rad fahrende Urlauber. Die mit Gras bewachsene Deichkrone liegt 6 m über mittlerem Hochwasser. Nördlich von Wyk auf Föhr (April 2004).

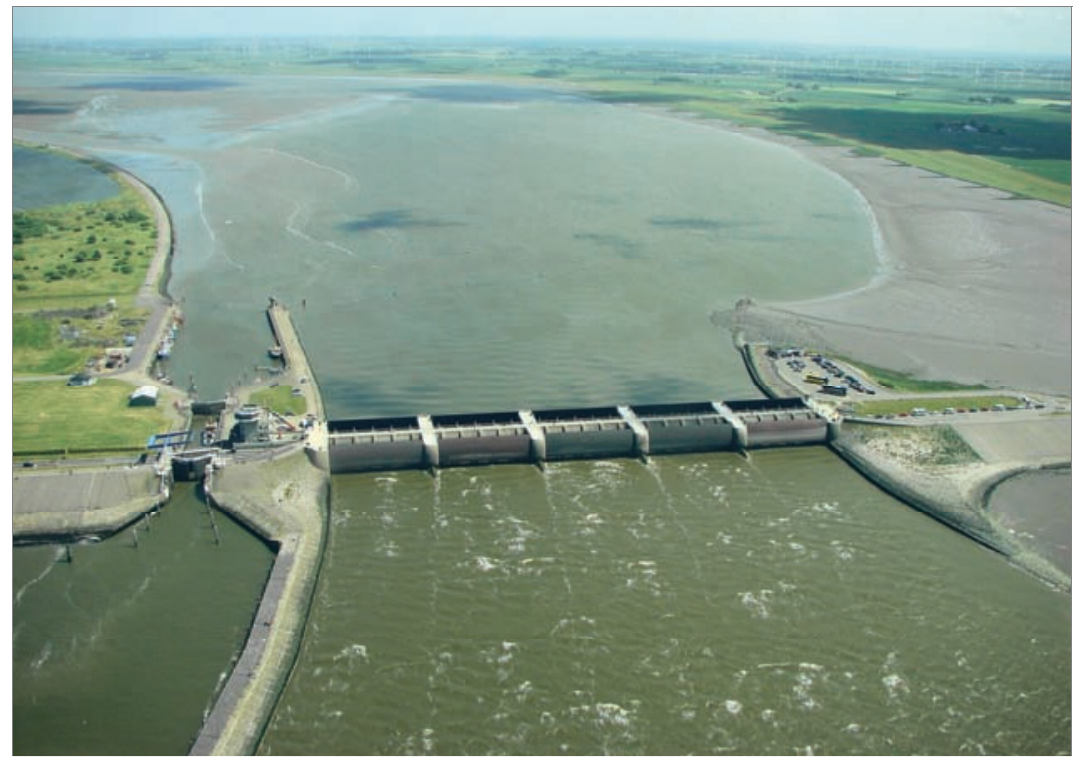

Abb. 7: Konfrontation zwischen Meer und Fluss: Eidersperrwerk bei ablaufendem Wasser und mit bei Sturmflut verschließbaren Toren, links eine Schiffsschleuse (Juni 2009). 
Doch beginnend in den 1960er Jahren bahnte sich eine ganz neue Entwicklung an.12 Für zusätzliche Flächen zur Erhöhung der landwirtschaftlichen Produktion war kein wirtschaftlicher Bedarf mehr. Neue Deiche wurden nur noch zur Verbesserung des Küstenschutzes gebaut, nicht mehr für die Landgewinnung. KostenNutzen-Berechnungen führten zur Aufgabe der Pläne, noch mehr Watt einzudeichen. Die Menschen begannen sich hinter den Deichen immer sicherer vor Sturmfluten zu fühlen, während gleichzeitig die Sorge um die Natur wuchs. Schadstoffbelastungen, übermäßige Bejagung und Überfischung und die Überdüngung der Gewässer wurden zunehmend zum beherrschenden Thema an der Küste. Jeder weitere Verlust an natürlichen Salzwiesen und Wattflächen rief Empörung hervor. In der Wahrnehmung hatte der Wert verbliebener Naturflächen den von landwirtschaftlichen Flächen überflügelt. Watt erhielt ein positives Image. Intakte Natur wurde für den aufblühenden Tourismus auf den Wattenmeerinseln zum Wirtschaftsfaktor. Die Küste wurde nicht nur wegen der Strände und der salzigen Luft als erholend empfunden, sondern auch die Vogelschwärme, Seehunde und Wattwanderungen erhöhten die Attraktivität. Zahlreiche Bücher und Naturfilme über die ursprüngliche Natur und Dynamik des Wattenmeers trugen zum Wertewandel bei. Seit 1978 verfolgen die Niederlande, Deutschland und Dänemark eine gemeinsame Naturschutzpolitik für die gesamte Wattenmeerregion. Gegen heftige Kontroversen wurde in Deutschland das Wattenmeer zum Nationalpark erklärt (1985/86). Das niederländische und deutsche Wattenmeer wurde 2009 zum Weltnaturerbe und der dänische Teil soll 2014 folgen. Das hat in der Küstenbevölkerung, die zuvor dem Naturschutz überwiegend skeptisch begegnete, einen Sinneswandel ausgelöst. Die Mehrheit ist heute stolz, dass ihre Küste als Weltnaturerbe ausgezeichnet wurde.

\footnotetext{
12 Wolff WJ (1992) The end of a tradition: 1000 years of reclamation of wetlands in The Netherlands come to an end. Ambio 21. S. 287-291; Kabat P, Bazelmans J, van Dijk J et al. (2012) The Wadden Sea region: Towards a science for sustainable development. Ocean \& Coastal Management 68. S. 4-17.
} 


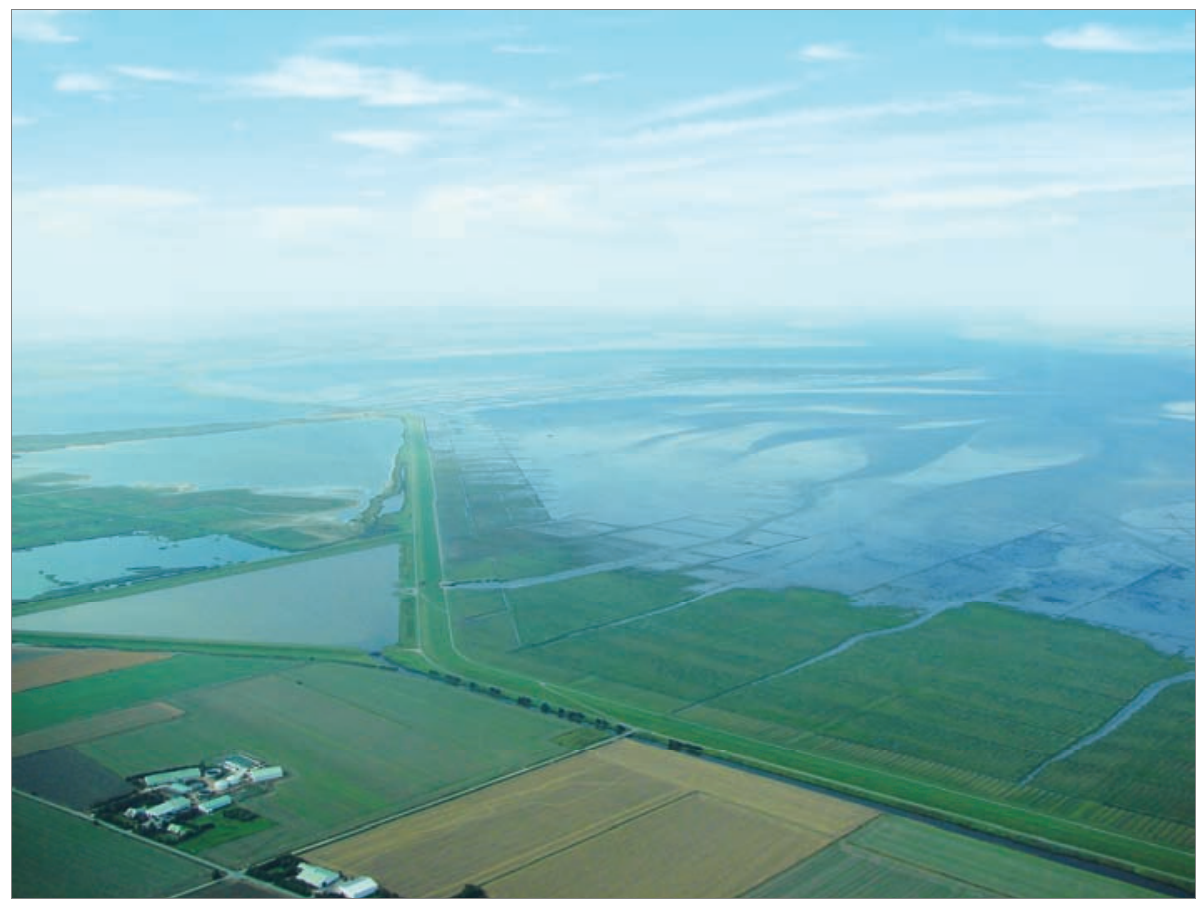

Abb. 8: Die letzte Großeindeichung 1987 von Salzwiesen und Watt war heftig umstritten. Der neue Deich verbessert den Küstenschutz und der entstandene Beltringharder Koog verbessert die Entwässerung von tief liegendem Hinterland und dient dem $\mathrm{Na}$ turschutz als Ausgleich für den Eingriff ins Wattenmeer. Vorne ein Gehöft im 1926 eingedeichten Sönke-Nissen-Koog. Das Deichvorland entstand durch Verlandungsarbeiten, die rechts im Bild aus Naturschutzgründen nicht mehr fortgeführt werden (August 2009).

Damit hat sich die Küstenmentalität innerhalb von rund 30 Jahren fundamental gewandelt. Aus einem weitgehend als öde und nutzlos wahrgenommenen Watt und einem bedrohlichen Meer ist ein bedrohter Naturraum geworden, um dessen Schutz und Zukunft man sich sorgt. Wieder kann darin keine zwangsläufige Entwicklung gesehen werden, sondern sie ist mehr das Resultat des Zusammentreffens von drei unabhängigen Entwicklungen: (1) Es rechnete sich nicht mehr, dem Wattenmeer neues Land abzuringen, (2) die Menschen fühlten sich hinter den massiven, neuen Deichen nicht mehr so vom Meer bedroht und konnten es nun, sensibilisiert durch die weltweite Umweltkrise, als bedroht wahrnehmen, (3) der Tourismuswirtschaft kommt die Anerkennung des Wattenmeers als Weltnaturerbe entgegen. So hat sich eine neue Allianz für das Wattenmeer gebildet, die auf die künftige Entwicklung weiter Einfluss nehmen wird. 


\section{Wie natürlich ist die Wattnatur?}

Die Natur ist nicht als ein Ganzes zu begreifen und schon gar nicht als etwas, was außerhalb von uns Menschen liegt, denn wir selbst sind ohne Zweifel Natur, wenn wir husten, uns vermehren und auch dann, wenn wir unseren Verstand benutzen. Zwar war der Mensch nicht von Anfang an bei der Geschichte der Natur dabei, aber trotz seines späten Auftritts hat er eine beispiellose Karriere hingelegt und dabei nicht nur das übrige Ensemble gründlich aufgemischt, sondern auch das Bühnenbild neu arrangiert. Das gilt auch für seine Rolle im Wattenmeer, wo er zudem von Anfang an dabei war. Von den großen Tieren, die im Doggerland mit den Menschen lebten, ist kaum etwas in der heutigen Küstenregion verblieben und viele sind sogar ganz ausgestorben.

Auch die Meeresfauna hat wahrscheinlich schon früh den Menschen zu spüren bekommen. ${ }^{13}$ Sehr wahrscheinlich schwammen Grauwale durch die tiefen Rinnen des Wattenmeers auf ihren saisonalen Wanderungen zwischen afrikanischer Küste und Barents-See. Durch ihre besondere Nähe zum Ufer, dürften sie unter allen Walen die leichteste Beute gewesen sein. Heute gibt es sie nur noch im nördlichen Pazifik. Die kleinen Schweinswale wurden viel in alten Berichten erwähnt und waren wahrscheinlich häufiger als heute. Nach Knochenfunden zu urteilen, waren Kegelrobben im Wattenmeer bis ins Mittelalter häufiger als Seehunde. Sie warfen im Winter ihre Jungen im oberen Strandbereich, wo sie leicht zu erschlagen waren. Seehunde bevorzugen hingegen flache, entlegene Sandbänke. Auf sie nahm der Jagddruck Anfang des 20. Jahrhunderts besonders zu, als organisiertes Jagdvergnügen für Touristen. Schadstoffe beeinträchtigten außerdem ihre Fortpflanzung. Jagdverbot und reduzierte Gifteinträge führten ab den 1970er Jahren zu einer 10-fachen Zunahme der Population, trotz zweier von Norden her eingeschleppter Virusepidemien. Kegelrobben sind seit dem Ende der Robbenjagd auch wieder im Wattenmeer heimisch. Bei beiden Arten hat die Fluchtdistanz stark abgenommen, so dass sie zu einer wichtigen touristischen Attraktion geworden sind.

\footnotetext{
13 Wolff WJ (1998) Causes of extirpations in the Wadden Sea, an estuarine area in The Netherlands. Conservation Biology 14. S. 876-885; Lotze HK (2005) Radical changes in the Wadden Sea fauna and flora over the last 2,000 years. Helgoland Marine Research 59. S. 71-83.
} 




Abb. 9: Schematische Darstellung von Verlust und Gewinn seit Entstehen des Wattenmeers für ausgewählte Tiergruppen. Während Jagd und Fischerei schon früh zu Verlusten führten, war nach 1900 der Naturschutz bei Meeressäugern und Küstenvögeln erfolgreich, aber bei Fischen bisher nicht. Raubbau löschte den Bestand einheimischer Austern aus, aber eine eingeführte Kulturauster verwilderte und überwächst Miesmuschelbänke.



Abb. 10: Ein Ausflugsschiff setzt Wattwanderer am Japsand ab und im Vordergrund rasten ungestört Seehunde (Juli 2012). 
Krauskopfpelikane gehörten ursprünglich zur Vogelfauna im Wattenmeer. Viele am Boden brütende Seevögel wie Eiderenten, Möwen und Seeschwalben wurden stark durch Eiersammeln dezimiert. Gänse, Enten, Kormorane und Watvögel wurden intensiv bejagt. Die daraus resultierende Vogelarmut der Küste rief zu Beginn des 20. Jahrhunderts den Vogelschutz auf den Plan. Vogelwarte beschützten Brutkolonien und ab den 1970er Jahren wurde nach und nach die Bejagung im Wattenmeer eingestellt und die Vogelbestände erholten sich. Sogar Greifvögel wie Wanderfalke und Seeadler sind wieder gekommen.

Bei den Zugvögeln zwischen arktischen Brutgebieten und der Überwinterung in Westafrika stößt Vogelschutz noch an seine Grenzen. Aber z. B. Ringelgänse wissen offenbar, wo sie gejagt werden und wo nicht und verhalten sich entsprechend.

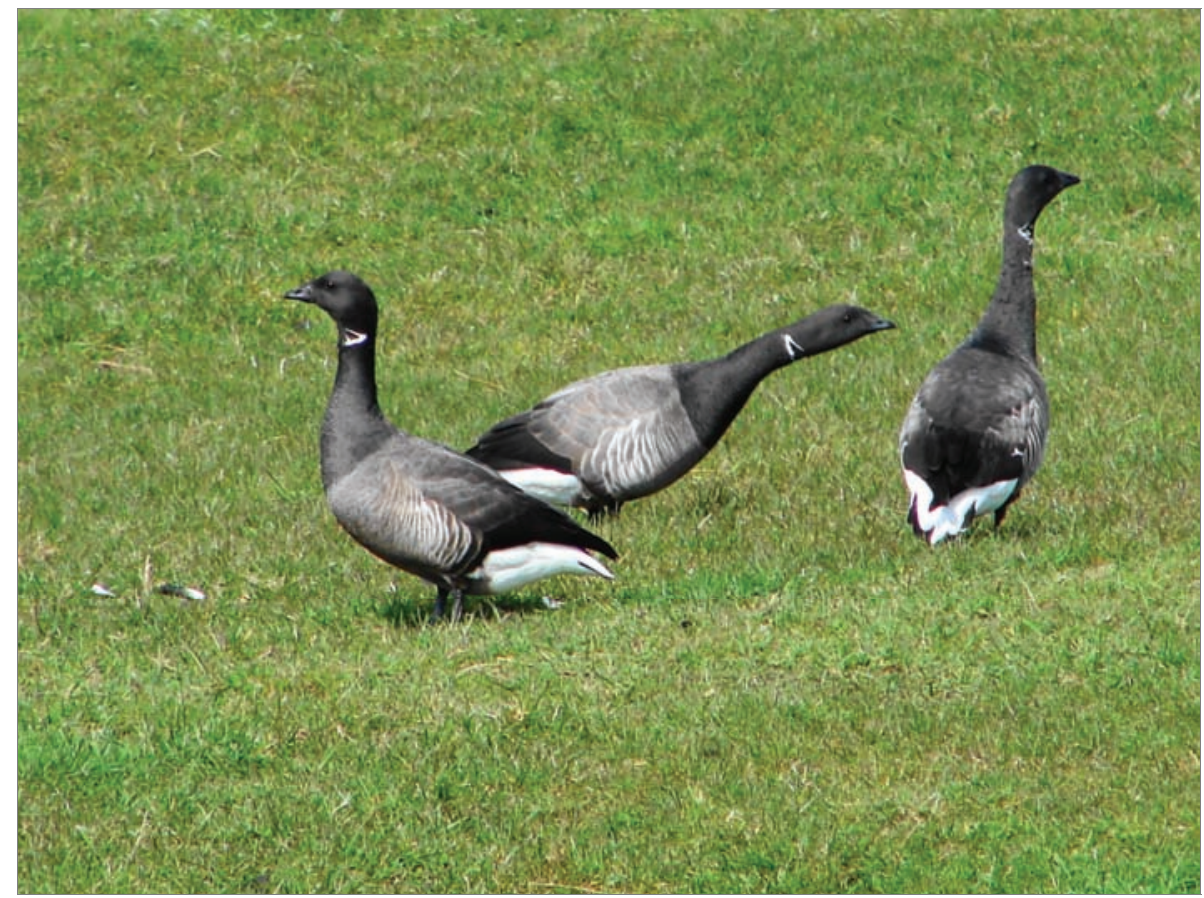

Abb. 11: Ringelgänse auf den Weiden von Hallig Langeness fressen sich ohne Scheu dort Fettpolster für den Weiterflug ins Brutgebiet an der sibirischen Küste an (April 2012).

Inszenierte Ringelganstage im April locken heute zahlreiche Besucher auf die Halligen in Nordfriesland. Vogelschutz und Robbenschutz im Wattenmeer wurde zur Erfolgsgeschichte.

Hinweise auf rückläufige Fischbestände oder abnehmende Größen gefangener Fische gibt es seit dem 17. Jahrhundert, aber ob der Grund Überfischung war, ist nicht sicher. Dies zeigt sich aber deutlich Ende des 19. und im 20. Jahrhundert. 
Besonders betroffen sind Fische, die in den Flüssen laichen, wie Stör, Lachs, Meerforelle, Schnäpel, Maifisch und Neunaugen. Verschlechterte Lebensbedingungen in den Flüssen und Überfischung wirkten hier zusammen. Auch das Vorkommen der Aale im Wattenmeer ist von den Beeinträchtigungen in Flüssen und Seen betroffen. Die Abdämmung der Zuiderzee führte dort zum Verlust zahlreicher Brackwasserfische. Große Rochenarten gerieten in der Nordsee in die Netze, noch bevor sie ein vermehrungsfähiges Alter erreichen konnten. Sie waren einst bedeutend für die Fischerei im Wattenmeer. Invasionen junger Kabeljau ins Wattenmeer treten nicht mehr auf, weil der gesamte Nordseebestand überfischt ist. Im Wattenmeer lohnt gewerbsmäßiger Fischfang nicht mehr. Nur der Fang von Garnelen (Krabben, Granat) ist noch von Bedeutung und Miesmuscheln von Bodenkulturen werfen noch Gewinne ab.

Besonders im nördlichen Wattenmeer lagen Austernbänke im flachen Wasser, die von großer wirtschaftlicher Bedeutung waren. ${ }^{14}$ Begrenzend für die Nutzung war lange Zeit der aufwändige Transport der empfindlichen Schalentiere zu den entfernten Märkten. Als das Eisenbahnnetz im 19. Jahrhundert ausgebaut wurde, verbesserte sich die Vermarktung. Allerdings zeigte sich schnell, dass die natürliche Vermehrung der Austern der gestiegenen Nachfrage nicht gewachsen war. Die Fangerträge brachen ein und Anfang des 20. Jahrhundert lohnte die kommerzielle Nutzung schon nicht mehr. Seit den 1950er Jahren ist die Ostrea edulis im Wattenmeer ausgestorben.

14 Reise K (1998) Einstige Austernbänke. In: Landesamt für den Nationalpark SchleswigHolsteinisches Wattenmeer, Umweltbundesamt (ed) Umweltatlas Wattenmeer Bd. 1 Nordfriesisches und Dithmarscher Wattenmeer. Ulmer, Stuttgart. S. 168-169. 


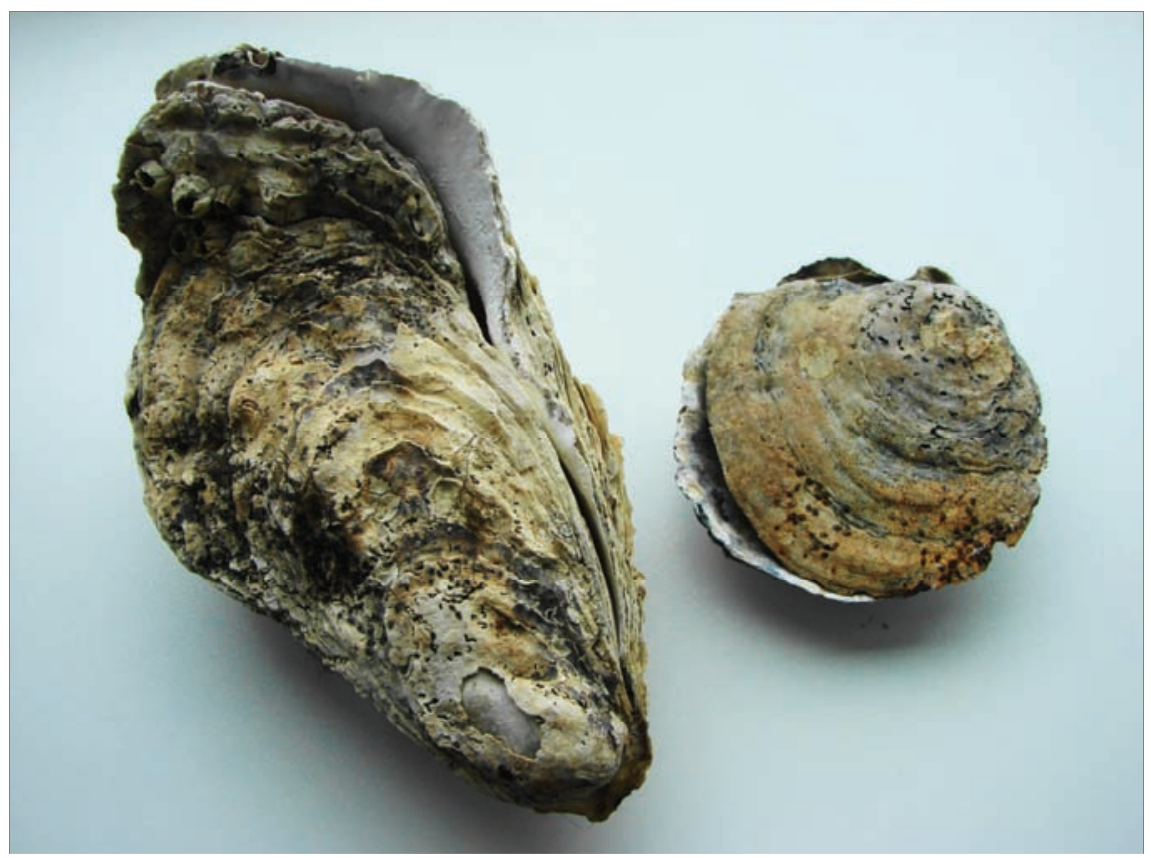

Abb. 12: Austernschalen: rechts die flache, einheimische Ostrea edulis und links die stärker gewölbte, aus dem Nordpazifik stammende Crassostrea gigas, die seit 1986 bei Sylt in Kultur genommen wurde und dann verwilderte.

Obwohl die damalige Fangstatistik keinen Zweifel daran lässt, dass Raubbau zum Verlust dieser Austern führte, werden gerne Krankheiten und strenge Winter als Ursache angeführt. Das ist jedoch ganz unwahrscheinlich, bei einer Art, die schon Jahrtausende im Wattenmeer überlebte. Heute gibt es wieder Austern im Wattenmeer. Dabei handelt es sich um Pazifische Austern, die als Ersatz eingeführt wurden und sich sehr erfolgreich im Wattenmeer ausbreiteten. Sie überwachsen seit den 2000er Jahren die Miesmuschelbänke im Gezeitenbereich, besetzten aber bisher nicht die ehemaligen Bänke der heimischen Austern.

Außer der Pazifischen Auster sind zahlreiche weitere Wirbellose und Pflanzen aus Übersee ins Wattenmeer eingeschleppt worden, meist als blinde Passagiere mit dem weltweiten Schiffsverkehr. In der historischen Bilanz gab es viele Verluste bei großen Tieren im Wattenmeer, während kleine im Zuge der maritimen Globalisierung hinzugekommen sind. Die Lebensgemeinschaften im Wattenmeer haben sich also durch Einwirkungen des Menschen im Verlauf der Zeit erheblich verändert. Durch die globale Erwärmung werden künftig mehr Arten aus Süden zuwandern. Was also ist natürlich im Wattenmeer?

Die Frage führt in ein unentwirrbares Knäul von direkten und indirekten, von prähistorischen bis zu aktuellen und von lokalen bis zu globalen Auswirkungen 
menschlichen Handelns auf die Komponenten der Natur. Außerdem entstammt die Frage noch der Vorstellung von einer ursprünglichen, quasi paradiesischen Natur: Bevor der Mensch in sie eintrat, befand sie sich noch im harmonischen Gleichgewicht der Naturkräfte, das sich trotz gelegentlicher Störungen selbst zu erhalten vermochte. Doch schon bevor der Mensch hinzutrat, veränderten sich fortlaufend die Lebensgemeinschaften, allmählich und durch qualitative Umbrüche. Oft wird in dieses vermeintliche Gleichgewicht noch der vorindustrielle Mensch mit hineingedacht. Das allerdings wäre schierer Unsinn, angesichts der schon vom frühen Menschen ausgerotteten Megafauna und der gelegten Feuer mit den Folgen für die Vegetation und Fauna. Durch die exponentielle Abnahme unseres Wissens mit der Ferne der Vergangenheit, können wir kaum irgendwo sicher sein, noch „ursprüngliche“ oder ,reine“ Natur vorzufinden - außer in ihren einzelnen Teilen, wie Arten, Symbiosen oder mäandrierenden Flussläufen. Das natürliche Gleichgewicht ist mehr ein Geist aus der Vergangenheit und ein esoterischer Wunsch von einem Paradies, das es auch vor dem Menschen wohl nicht gegeben hat.

\section{Welche Zukunft hat das Wattenmeer?}

In den letzten Jahren haben sich die Vorstellungen vom Wattenmeer grundlegend gewandelt. Es als ein Weltnaturerbe zu betrachten wirft die Frage auf, wie und in was für einem Zustand diese Küstenzone kommenden Generationen vererbt werden könnte. Frühere Zustände wären vielleicht wünschenswerter als der gegenwärtige, aber wie wir schon sahen, ist das angesichts irreversibler Veränderungen nicht möglich. An den Ufern des Wattenmeers wohnen viele Menschen und noch mehr Besucher kommen jedes Jahr zur Erholung, Schiffe bringen neue Organismen, das wärmer werdende Klima lockt Arten aus dem Süden an und der Meeresspiegel könnte wieder so schnell steigen wie einst nach der Eiszeit. Eine konservierte Gegenwart an die Kinder und Enkel weitergeben zu wollen, wie das bei einem Weltkulturerbe gedacht ist, wäre eine Illusion. Selbst wenn dies möglich wäre, ist doch wahrscheinlich, dass niemand unsere Gegenwart in der Zukunft haben will.

Was wäre wünschenswert? Zum Weltnaturerbe avancierte das Wattenmeer wegen seiner weltweit herausragenden Naturwerte, die nach menschlichem Ermessen auch in Zukunft als wertvoll erachtet werden könnten. Trotz Anstieg des Meeres wird kaum jemand wegziehen wollen. Kann es gelingen die Naturwerte zu erhalten und die Küste an höhere Wasserstände anzupassen? Eine Küstenlandschaft oder ein Ökosystem Wattenmeer ist nicht wie ein Kunst- oder Bauwerk, sondern lebendig und damit veränderlich. Ein Ökosystem funktioniert auch nicht wie eine geölte Maschine, in der alle Teile nach einem Konstruktionsplan so ineinander greifen, dass die gewünschte Leistung heraus kommt. Ein Ökosystem funktioniert auch nicht wie ein Organismus, in dem die Organe gemäß den Erbinformationen wachsen und so miteinander verbunden sind, dass ein Individuum erfolgreich seine Gene an die nächste Generation weitergeben kann. 
Solche Analogien verleiten zu einer Fürsorge, die repariert, saniert und stabilisiert. Durch Rückkopplungen führt das in immer enger werdende Abhängigkeit, die Gefahr läuft im Kollaps zu enden, wenn sich Rahmenbedingungen ändern oder Neues hinzukommt. Wird dagegen das ökologische Gefüge mehr als eine flexible Pluralität von individuellen Organismen wahrgenommen, in der nicht immer und nicht überall alles mit allem zusammenhängt, sondern auch Lücken klaffen können und alternative Verbindungen möglich sind, aus denen sich fortwährend Neues entwickelt, dann kann besser ein Abstand gewahrt werden. Solch eine Zurücknahme eröffnet die Freiheit über Debatten und Konsens zu entscheiden, wann und wo aus welchem Interesse Eingriffe, Schutz oder Hilfen erfolgen sollen. Diesen Abstand zu wahren und eine Offenheit gegenüber freien Entwicklungen auszuhalten, fällt uns schwer.

Kontingenz ermöglicht Freiheit, während die abstrakten, invarianten Naturgesetze mit ihren notwendigen Folgen in das Repertoire der Naturbeherrschung passen. Der Naturschutz sollte davon Abstand nehmen, Festschreibungen vermeiden und den Entwicklungen in der Naturlandschaft freien Lauf lassen. Dies ergibt sich aus der Naturgeschichte des Wattenmeers, wo die Komponenten der Natur vom Wirken der Menschen seit Anbeginn erst empfindlich bedrängt und heute zum Teil geschützt werden.

Steigt das Meer immer höher im Zuge der klimatischen Erwärmung, kann die Bevölkerung entweder durch immer martialischere Bollwerke gegen die See verteidigt werden oder sie versucht, mit mehr Wasser in der Landschaft zu leben. ${ }^{15}$ Wenn Siedlungen auf schwimmende Pontons verlegt würden, wenn von Land- auf Wasserwirtschaft umgestellt würde, dann könnte auch Meerwasser kontrolliert durch Deiche hindurch gelassen und zu einem fließenden Element in der Landschaftsgestaltung werden. Ein solches Küstenleben würde teils alte Traditionen wieder aufgreifen, aber auch ganz futuristische Konzepte erfordern. Eine solche, für Meerwasser durchlässige Küste, könnte mit dem steigenden Meer mitwachsen und sich so an die neuen Bedingungen anpassen können, statt sie nur abzuwehren. In diesem Fall hätte auch das Wattenmeer eine Zukunft und seine Naturgeschichte könnte weiter gehen.

\section{Literatur}

Bantelmann A (1992) Landschaft und Besiedlung Nordfrieslands in vorgeschichtlicher Zeit. Stiftung Nordfriesland, Föhr.

Behre KE (2008) Landschaftsgeschichte Norddeutschlands. Wacholtz Verlag, Neumünster.

\footnotetext{
${ }^{15}$ Reise K, Fischer L (2011) Unbequeme Überlegungen: Zur Geschichte der Küstenmentalitäten und den Herausforderungen durch den Klimawandel. In: L. Fischer, K. Reise (ed) Küstenmentalität und Klimawandel. Oekom, München. S. 199-217.
} 
Bunzel-Drüke M, Drüke J, Vierhaus H (1994) Quaternary Park. Überlegungen zu Wald, Mensch und Megafauna. ABUinfo 17/18. S. 1-35.

Gaffney V, Fitch S, Smith D (2009) Europe's lost world. The discovery of Doggerland. Council for British Archaeology, York.

Gould SJ (1989) Wonderful life. The Burgess shale and the nature of history. Hutchinson Radius, London.

Hampe M (2011) Tunguska oder das Ende der Natur. Carl Hanser Verlag, München.

Herrmann B (2009) Umweltgeschichte Wozu? Zur gesellschaftlichen Relevanz einer jungen Disziplin. In: P. Masius, O. Sparenberg, J. Sprenger (ed) Umweltgeschichte und Umweltzukunft. Universitätsverlag Göttingen, Göttingen. S. 13-50.

Kabat P, Bazelmans J, van Dijk J et al. (2012) The Wadden Sea region: Towards a science for sustainable development. Ocean \& Coastal Management 68. S. 4-17.

Lepenies W (1976) Das Ende der Naturgeschichte. Hanser Verlag, München, Wien.

Lotze HK (2005) Radical changes in the Wadden Sea fauna and flora over the last 2,000 years. Helgoland Marine Research 59. S. 71-83.

Oost AP, Hoekstra P, Wiersma A et al. (2012) Barrier island management: Lessons from the past and directions for the future. Ocean \& Coastal Management 68. S. $18-38$.

Reise K (1998) Einstige Austernbänke. In: Landesamt für den Nationalpark Schleswig-Holsteinisches Wattenmeer, Umweltbundesamt (ed) Umweltatlas Wattenmeer Bd. 1 Nordfriesisches und Dithmarscher Wattenmeer. Ulmer, Stuttgart. S. 168-169.

Reise K, Baptist M, Burbridge P et al. (2010) The Wadden Sea - A universally outstanding tidal wetland. Wadden Sea Ecosystem 29. Common Wadden Sea Secretariat, Wilhelmshaven.

Reise K, Fischer L (2011) Unbequeme Überlegungen: Zur Geschichte der Küstenmentalitäten und den Herausforderungen durch den Klimawandel. In: L. Fischer, K. Reise (ed) Küstenmentalität und Klimawandel. Oekom, München. S. 199-217.

Wolff WJ (1992) The end of a tradition: 1000 years of reclamation of wetlands in The Netherlands come to an end. Ambio 21. S. 287-291.

Wolff WJ (1998) Causes of extirpations in the Wadden Sea, an estuarine area in The Netherlands. Conservation Biology 14. S. 876-885. 



\title{
Der Kojenmann \\ Mensch und Natur im Wattenmeer, 1860-1900
}

\author{
Martin Rheinheimer
}

Amrum ist eine karge Insel im schleswig-holsteinischen Wattenmeer. Die etwa $20 \mathrm{~km}^{2}$ große Insel liegt südlich von Sylt und westlich von Föhr. Ende des 19. Jahrhunderts hatte sie noch ein etwas anderes Äußeres als heute, denn der breite Kniepsand, der heute den Dünen im Westen vorgelagert ist, hatte noch nicht auf ganzer Länge eine feste Verbindung mit der Insel. Ursprünglich war er ein Hochsand, der westlich von Amrum in der Nordsee lag. Der Wind ließ ihn jedoch nach Osten wandern, und so kam er zunächst im Süden in Verbindung mit der Insel. Zwischen Sand und Dünen entstand eine geschützte Bucht, der Kniephafen. Dieser versandete im Laufe des 19. Jahrhunderts, als der Sand weiterwanderte. Ende des 19. Jahrhunderts gab es die Bucht aber noch, auch wenn sie immer kleiner wurde. ${ }^{1}$

Mit den Napoleonischen Kriegen und der Kontinentalsperre ging die Seefahrtsperiode der Nordfriesischen Inseln ihrem Ende zu. Hatte der Dienst auf holländischen und hamburgischen Walfängern den kargen und übervölkerten Inseln im 18. Jahrhundert Wohlstand beschert, so musste man sich im 19. Jahrhundert nach neuen Einnahmequellen umsehen. Ein Teil der Männer wandte sich der Langfahrt zu: Man segelte jetzt nicht mehr von Frühling bis Herbst, sondern ging auf lange Seereisen in der Handelsfahrt. ${ }^{2}$ Dadurch änderte sich der Rhythmus von Abreise und Rückkehr der Männer, die früher für alle zu festen Zeitpunkten erfolgt war. Auf Föhr wandte man sich der Landwirtschaft zu, die die Föhrer Männer bislang den Frauen und

\footnotetext{
${ }_{1}^{1}$ Quedens G, Titzck R (1991) Amrum. Landschaft - Geschichte - Natur. Amrum.

2 Rheinheimer G (2012) Nordfriesische Seeleute in der Amsterdamer Handelsfahrt. In: Zeitschrift der Gesellschaft für Schleswig-Holsteinische Geschichte 137. S. 31-77. S. 31-77.
} 
fremden Saisonarbeitern überlassen hatten, um sich der ertragreicheren Seefahrt zu widmen. ${ }^{3}$ Auf Amrum bestand diese Möglichkeit jedoch nicht in gleichem Maße, da die Insel vor allem aus Dünen und Heide bestand.

Die Insel bestand Mitte des 19. Jahrhunderts aus drei Dörfern: Norddorf, Nebel und Süddorf. Bei Steenodde standen nur einzelne Häuser. Die Insel hatte 1860 660 Einwohner. ${ }^{4}$ Der Tourismus hatte noch keinen Einzug gehalten, denn erst im Jahre 1890 wurde das Seebad Wittdün angelegt, wobei in der Folge boomartig ein ganzer Ort aus dem Boden gestampft wurde. ${ }^{5}$ Wie also konnten sich die Menschen in dieser Übergangsperiode zwischen Seefahrt und Tourismus ernähren? Viele wanderten in dieser Zeit nach Amerika aus. Die übrigen mussten mit dem auskommen, was ihnen zur Verfügung stand - und das war die Natur. Das Wattenmeer bot gewisse Möglichkeiten. Es soll im Folgenden untersucht werden, wie ein Tagelöhner von ihnen leben konnte.

Glücklicherweise ist Amrum im 19. Jahrhundert relativ quellenreich. Es existieren in Privatbesitz und in lokalen Archiven etliche Tagebücher und Rechnungsbücher, die uns genauen Aufschluss über die Wirtschaftsweise ihrer Verfasser geben können. Im Folgenden wird ein solches „Tagebuch“, das Cornelius Peters in den Jahren 1867 bis 1891 geführt hat, ausgewertet. ${ }^{6}$ Das Buch gibt einen guten Einblick in die gegenseitige Abhängigkeit von Natur, Wirtschaft und Gesellschaft in der zweiten Hälfte des 19. Jahrhunderts und die auf Naturausnutzung basierte kombinierte Wirtschaftweise. Peters lebte von Vogelfang, Fischerei, Jagd und Tagelohn. Im Zentrum soll also die Frage stehen: Wie konnte eine Familie auf Amrum von der umgebenden Natur leben?

Cornelius Wilhelm Peters wurde am 30. Oktober 1836 in Norddorf auf Amrum geboren. Sein Vater Wilhelm Roluf Peters war im April 1834 von Utersum auf Föhr nach Norddorf gezogen und betrieb dort einen Laden, später auch eine Gastwirtschaft. Als Bauernvogt (1854), Birksrepräsentant (1856), Strandvogt (1861) und Schulvorsteher (1862) war der Vater einer der Dorfhonoratioren. ${ }^{7}$ Cornelius Peters erreichte nie den Status seines Vaters, sondern blieb im Prinzip ein Tagelöhner. Er starb, nachdem er ein Jahr lang gekränkelt hatte, am 24. August 1892 in Norddorf, 55 Jahre alt. ${ }^{8}$

Cornelius Peters wurde 1869 von den Anteilseignern der Vogelkoje als Kojenwart angestellt. Die Vogelkoje war eine Entenfanganlage. Um einen Teich waren

\footnotetext{
3 Roeloffs BC (1985) Von der Seefahrt zur Landwirtschaft. Ein Beitrag zur Geschichte der Insel Föhr. Wachholtz, Neumünster.

${ }_{4}^{4}$ Remde F (1972) Amrum. Ein Beitrag zur Genese und Struktur einer Inselsiedlung. Diss. Univ. Münster, Münster.

${ }^{5}$ Quedens G (1990) Das Seebad Amrum. Amrum.

${ }^{6}$ Das Tagebuch befindet sich im Besitz von Julius Peters, Steenodde. Georg Quedens hat Auszüge daraus publiziert: Quedens G (1986) Tagebücher aus dem alten Amrum. Amrum. S. 113-124. Die Auszüge sind jedoch nicht wortgetreu, sondern nur sinngemäß und mit erheblichen Auslassungen. Eine ausführliche Auswertung findet sich in: Rheinheimer M (2007) Der Kojenmann. Mensch und Natur im Wattenmeer 1860-1900. Wachholtz, Neumünster.

${ }^{7}$ Kirchenkreisarchiv Leck, Kirchenbuch St. Clemens/Amrum Nr. 1.9. F. 158v, 159r.

${ }^{8}$ Kirchenkreisarchiv Leck, Kirchenbuch St. Clemens/Amrum Nr. 1.10. S. 483f.
} 
vier Pfeifen gruppiert, die mit Netzen überspannt waren und die in Reusen mündeten. Während des Vogelzuges ließen sich Enten auf dem Teich nieder und wurden mit Hilfe von zahmen Lockenten in die Pfeifen gelockt. Dort wurden sie vom Kojenwart gefangen und getötet. Die ersten Vogelkojen auf den Nordfriesischen Inseln wurden im 18. Jahrhundert nach niederländischem Vorbild eingerichtet. Auf Amrum wurden in der zweiten Hälfte des 19. Jahrhunderts zwei solche Anlagen gebaut, eine im Norden der Insel bei Norddorf und eine im Süden bei Wittdün. ${ }^{9}$ Peters war in der Koje Meeram bei Norddorf tätig.

Als Kojenwart war Peters zur Rechnungsführung verpflichtet, zumal er selbst Anteile am Fang erhielt. Jeder Monat erhielt in seinen Aufzeichnungen eine eng beschriebene Seite. Er notierte tabellenartig in eigenen Rubriken: 1) Wochentag, 2) Datum, 3) Bemerkungen, 4) Witterung und 5) Windrichtung. Die Bemerkungen enthalten Nachrichten über seine Arbeit und besondere Vorkommnisse meist familiärer Art. Während der Entensaison notierte er dazu für jeden Tag die Gesamtzahl der gefangenen Enten sowie die Anzahl jeder einzelnen Art. Am Ende jeder Seite steht die Gesamtzahl seiner Fänge (diese Monatssummen sind jedoch nicht immer vollständig). Am Jahresende folgt jeweils eine Seite mit seinen Einnahmen und Ausgaben. Es sind zwei Hefte erhalten. Das erste umfasst genau zehn Jahre und reicht von Januar 1867 bis Dezember 1876. Das zweite Heft reicht von März 1887 bis Februar 1891. Es ist leider in viel schlechterem Zustand als das erste, und es fehlen dadurch mitunter Angaben. So fehlt das Blatt mit August und September 1888. Beide Hefte sind ansonsten völlig identisch in ihrem Aufbau. Man muss deshalb davon ausgehen, dass ein drittes Heft, welches die fehlenden Jahre 1877 bis 1886 deckte, existierte, aber verloren gegangen ist. ${ }^{10}$

Cornelius Peters lebte von verschiedenen Arbeiten. Im Laufe der Jahre entwickelte er eine Art Jahreszyklus. Bestimmte Arbeiten kehren Jahr für Jahr ungefähr zur gleichen Zeit wieder. Man kann gut erkennen, wie sich Cornelius Peters bald nach seiner Heirat ${ }^{11}$ eine eigene Existenz aufbaute: Er fand Arbeit an der neuen Vogelkoje, er baute sich einen Prahmen (= ein Boot), er setzte einen Fischgarten (eine Art Fischzaun, in dem sich mit Hilfe des Gezeitenstromes insbesonderen Plattfische fangen ließen). 1869 wurde er Kojenwart und hatte damit eine „feste“ Beschäftigung, von der er jedoch keineswegs ausschließlich leben konnte. Sie gab aber den festen Rahmen, der die anderen Tätigkeiten bestimmte.

Es lässt sich aus Cornelius Peters' Aufzeichnungen der in Tab. 1 dargestellte Jahreszyklus erstellen. Dabei lassen sich Haupt- und Nebenarbeiten unterscheiden. Hauptarbeiten sind solche, die für sein Gesamteinkommen am wichtigsten waren. Nebenarbeiten sind solche, die eine geringere Bedeutung hatten. Daneben stehen Tätigkeiten, die er nur in wenigen Jahren ausübte. Für bestimmte Tätigkeiten, die einen hohen Profit brachten, wie z. B. die Wache auf gestrandeten Schiffen, stellte

\footnotetext{
${ }^{9}$ Quedens (1990).

${ }^{10}$ Bereits Georg Quedens fand das Schreibebuch in diesem Zustand vor.

${ }^{11}$ Er heiratete am 23. Januar 1863. KKA Leck, Kirchenbuch St. Clemens/Amrum Nr. 1.9, f. 115v$116 r$.
} 
er oft auch die Haupttätigkeiten beiseite, doch nie seine Tätigkeit in der Vogelkoje. Die Strandung von Schiffen war jedoch unregelmäßig und lässt sich nicht in den Zyklus einordnen, da sie zu fast allen Jahreszeiten geschehen konnte.

Tab. 1: Cornelius Peters’ Arbeitszyklus, 1867-1890.

\begin{tabular}{|l|l|l|l|}
\hline Monat & Hauptarbeit & Nebenarbeit & $\begin{array}{l}\text { Arbeit in einzel- } \\
\text { nen Jahren }\end{array}$ \\
\hline Januar & Aalstechen & Kaninchenfang & \\
\hline Februar & Aalstechen & Kaninchenfang & \\
\hline März & Fischgarten (Schollen) & Kaninchenfang & \\
\hline April & Fischgarten (Schollen) & Wegearbeiten & \\
\hline Mai & Fischgarten (Schollen) & Eiersammeln, Heide & $\begin{array}{l}\text { zweite Hälfte: } \\
\text { Seefischerei }\end{array}$ \\
\hline Juni & Heide & Eiersammeln & \\
\hline Juli & Grasmähen (Tagelohn) & & \\
\hline August & $\begin{array}{l}\text { (Tagelohn), zweite Hälfte: } \\
\text { Vorbereitung der Vogelkoje }\end{array}$ & Kaninchenfang & \\
\hline September & Entenfang in der Vogelkoje & & Austernfang \\
\hline Oktober & Entenfang in der Vogelkoje & & Austernfang \\
\hline November & Entenfang in der Vogelkoje & Reetschneiden & Kaninchenfang \\
\hline Dezember & Aalstechen & & \\
\hline
\end{tabular}

Im Winter, von Dezember bis Februar, war Cornelius Peters hauptsächlich mit dem Aalestechen beschäftigt. Daneben fing er auch Kaninchen. Im Frühling, von März bis Mai, fing er mit dem Fischgarten vor allem Schollen. Daneben standen noch etwas Kaninchenfang und Wegearbeiten, im Mai begann dann auch das Eiersammeln und Heideschlagen. Der Juli war seit 1872/73 ausgefüllt mit dem Mähen von Wiesen im Tagelohn. Anfang August mähte er im Tagelohn Kornfelder. Dann wurde die Vogelkoje betriebsfertig gemacht, und es begann die Entenfangsaison, die bis zum November dauerte. Im November schnitt er meist auch einige Tage Reet.

Zählt man die Tage zusammen, an denen Cornelius Peters erfolgreich Aale, Schollen und Enten fing, so wird deutlich, dass jede dieser Tätigkeiten eine bestimmte Jahreszeit in Anspruch nahm: der Aalfang fand im Winter statt, der Schollenfang im Frühling, der Entenfang im Herbst (Abb. 1). Man könnte diese Graphik noch um weitere Tätigkeiten wie das Heu- und Kornmähen im Sommer ergänzen. Es handelt sich hier im Übrigen nur um die Tage, an denen er etwas fing. Daneben 
stehen selbstverständlich auch die Tage, an denen er die Reusen reparierte und die Vogelkoje in Stand setzte. Im August bereitete er die Vogelkoje erst für den Fang vor, im November musste er sie winterfest machen.

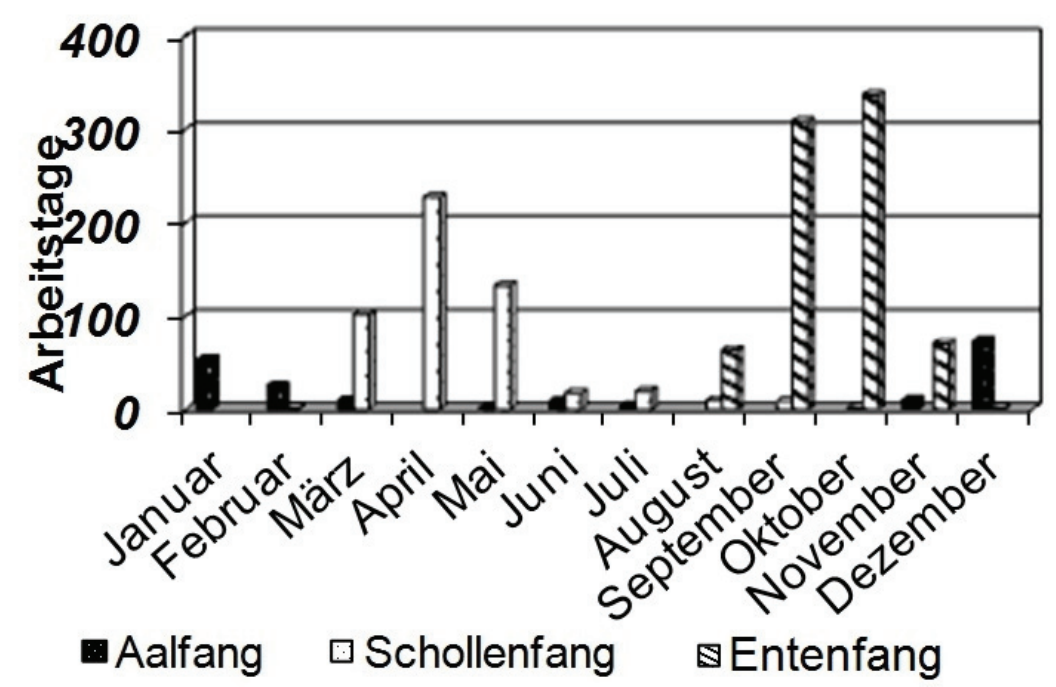

Abb. 1: Cornelius Peters' Arbeitsjahr 1867-1890.

Dieser Arbeitsrhythmus war ganz von der Natur bestimmt. Ähnlich wie der Rhythmus eines Bauern den Jahreszeiten folgte, die bestimmte Tätigkeiten wie Pflügen, Saat, Ernte und Dreschen vorgaben, so war auch der Jahreszyklus des Amrumer Tagelöhners von der Natur bestimmt. Nur war es hier weniger das Wachsen der Pflanzen, das den Zyklus bestimmte, sondern das Eintreffen der Zugvögel (Enten) und der Fische (Schollen, Aale). Die Ernte fügte sich relativ gut in diesen Zyklus ein, da im Juni/Juli die Fische verschwunden und die Enten noch nicht gekommen waren. Deshalb begann Cornelius Peters auch in 1870er Jahren im Sommer als Tagelöhner in der Ernte zu arbeiten. An Sturmtagen war er im Winter oft mit Strandgut beschäftigt, bei gutem Wetter mit Aalen.

Seit 1872 verdiente Cornelius Peters im Sommer Geld bei der Ernte. Damals mähte er im Juli für seinen Vater, im November und Dezember schnitt er auch Reet für diesen. Der Vater war zu diesem Zeitpunkt offenbar bereits geschwächt. Er starb im Januar. Seit 1873 arbeitete Cornelius nun in der Ernte für andere. Dabei gewann er bald einen festen Kundenkreis und es entstand ein fester Arbeitsrhythmus für den Sommer. Und zwar mähte er von Ende Juni oder Anfang Juli bis Ende Juli Wiesen. Zwischendurch mähte er auch zwei bis drei Tage das Gras in der Vogelkoje, und richtete diese bereits für den Fang her. Anschließend mähte er von Anfang bis Mitte August Korn und machte dann die Koje endgültig startklar (vgl. Tab. 2). 
Die Mahd der Wiesen nahm 19 bis 25 Tage in Anspruch, die Mahd der Kornfelder 8 bis 11. Dabei handelt es sich allerdings nicht immer um volle Arbeitstage, sondern es sind die Tage, an denen er mähte.

Tab. 2: Cornelius Peters' Sommerarbeit, 1873-1876.

\begin{tabular}{|l|ll|ll|}
\hline Jahr & $\begin{array}{l}\text { Wiesenmahd } \\
\text { (Tagelohn) }\end{array}$ & $\begin{array}{l}\text { Arbeitstage im } \\
\text { Tagelohn }\end{array}$ & $\begin{array}{l}\text { Kornmahd } \\
\text { (Tagelohn) }\end{array}$ & $\begin{array}{l}\text { Arbeitstage im } \\
\text { Tagelohn }\end{array}$ \\
\hline 1873 & 25. Juni - 1. August & 25 & 2.-11. August & 8 \\
1874 & 6. Juli - 1. August & 19 & 3.-13. August & 11 \\
1875 & 28. Juni - 29. Juli & 23 & 29. Juli - 7. August & 9 \\
1876 & 1. Juli - 1. August & 21 & 2.-12. August & 9 \\
\hline
\end{tabular}

Wie die Landwirtschaft, so mussten auch Fischerei und Jagd selbst an Sonn- und Feiertagen durchgeführt werden, wenn man davon leben können wollte. Auch an Feiertagen musste der Fischgarten geleert werden, so an Ostern zumindest morgens. Das gleiche galt für die Kaninchenfallen, die selbst zu Weihnachten kontrolliert wurden. Dass das Wetter eine große Bedeutung für den Alltag eines Kojenmannes und Fischers hatte, ist offensichtlich. Cornelius Peters führte genau Buch. Er notierte jeden Tag das Wetter und schrieb auch die Windrichtung auf. Außerdem notierte er Besonderheiten wie Hochwasser oder das Nordlicht vom 22.-25. September und am 24./25. Oktober 1870.

Im Winter war deutlich weniger Arbeit, und er musste sich in dieser Jahreszeit selbst Arbeit schaffen. Dies konnte der Aalfang sein, der aber in der kalten Jahreszeit sehr ungesund war, später machte er Besen. Gerade für die Alten, die das Wetter nicht mehr ertrugen, war der Winter schwierig.

Cornelius Peters machte am Ende eines jeden Jahres eine genaue Abrechnung seiner Einnahmen und Ausgaben. Daraus lassen sich Gewichtungen, aber auch Veränderungen erkennen. Da im Jahre 1875 die Reichsmark eingeführt wurde, sind im Folgenden alle Werte einheitlich in Reichsmark umgerechnet.

Cornelius Peters' Gesamteinkommen schwankte in den überlieferten Jahren zwischen 408,58 RM im Jahre 1870 und 1.285,87 RM im Jahre 1876 (Tab. 6). Sein Einkommen war also in einzelnen Jahren dreimal so hoch wie in anderen (bzw. machte nur den dritten Teil aus). Der Durchschnitt lag bei 732,86 RM, und von diesem Mittelwert aus gesehen, waren die Ausschläge überbrückbar, zumal wenn man berücksichtigt, dass Cornelius Peters mit den meisten Lebensmitteln Selbstversorger war.

Man muss bei diesen Zahlen jedoch beachten, dass in der Jahresabrechnung nur die in Geld abrechenbaren Einkünfte auftauchen, d. h. die Fische oder Produkte, die er verkaufte. Darüber hinaus hatte er jedoch auch noch die rein naturale Ausbeute seiner Arbeit: z. B. die Fische, die er nicht verkaufte, sondern selber aß. Da ein Hauptanteil seiner Tätigkeit im Bereich Jagd und Fischerei lag, konnte er 
sich einen erheblichen Anteil seiner Lebensmittel auf diese Weise selbst beschaffen. Für sie tauchen weder Einnahmen noch Ausgaben in den Abrechnungen auf. Das Gleiche gilt für die Gegenstände, die er am Strand fand und für seinen eigenen Bedarf behielt. Auch sie trugen nicht unerheblich zu seiner Ökonomie bei, ohne jedoch in den Abrechnungen aufzutauchen.

Unter Cornelius Peters' in Geld spezifizierten Einnahmen machten Fischerei und Jagd mit im Durchschnitt $72 \%$ generell den bei weitem größten Posten aus (Tab. 7). Strandwesen und Bergelohn ergaben $13 \%$. In Landwirtschaft und Küstenschutz verdiente er $8 \%$ seines Einkommens, bei Wegearbeiten und anderen handwerklichen Arbeiten 3,8\%, im Tourismus 2,1\%.

Tab. 3: Die Fangsaison in der Vogelkoje, 1869-1890 (FS = Fangsaison).

\begin{tabular}{|cccccc|}
\hline Jahr & Beginn der FS & Ende der FS & Fangtage & Fang insges. & Fang pro Tag \\
\hline 1869 & 14. Sept. & 12. Nov. & 60 & 1047 & 17,5 \\
1870 & 9. Sept. & 31. Okt. & 53 & 3041 & 57,4 \\
1871 & 28. Aug. & 10. Nov. & 75 & 5724 & 76,3 \\
1872 & 12. Aug. & 5. Nov. & 86 & 7134 & 83,0 \\
1873 & 26. Aug. & 28. Okt. & 64 & 2004 & 31,3 \\
1874 & 23. Aug. & 9. Nov. & 79 & 4120 & 52,2 \\
1875 & 30. Aug. & 30. Okt. & 62 & 5505 & 88,8 \\
1876 & 24. Aug. & 18. Nov. & 87 & 11521 & 132,4 \\
& & & & & \\
1887 & 29. Aug. & 16. Nov. & 80 & 9813 & 122,7 \\
1888 & 30. Aug. & 16. Nov. & 79 & 11655 & 147,6 \\
1889 & 27. Aug. & 1. Nov. & 67 & 3273 & 48,9 \\
1890 & 25. Aug. & 31. Okt. & 68 & 6448 & 94,8 \\
\hline zusammen & & & 860 & 71285 & 82,9 \\
\hline
\end{tabular}

Schauen wir uns die einzelnen Jahre näher an, sind jedoch erhebliche Schwankungen zu erkennen. Die unter „Fischerei und Jagd“ subsummierten Einkünfte setzten sich aus verschiedenen Posten zusammen, von denen die Einkünfte aus der Vogelkoje den größten Anteil ausmachten. In den Jahren 1867 und 1868 waren diese Einkünfte jedoch noch gering. Es waren damals nur Gelegenheitsarbeiten, da er noch nicht als Kojenwart fest angestellt war. In diesen Jahren machten die Einkünfte aus der Vogelkoje nur 15,8 bzw. 3,0 \% seiner Einkünfte aus. In den folgenden Jahren wurde diese Einnahme jedoch diejenige, auf der seine Ökonomie beruhte. Bereits 1869 bezog er 53,7 \% seiner Einkünfte aus der Vogelkoje, und im Jahre 1870 waren es 84,4\%. Im Durchschnitt aller überlieferten Jahre machte die Vogelkoje 61,5 \%, also knapp ein Drittel seiner Einkünfte aus. 
Die Einkünfte aus der Vogelkoje waren jedoch weiterhin erheblichen Schwankungen unterworfen. Dies hing damit zusammen, dass er neben einem festen Salär für die Wartung der Vogelkoje Fangprämien erhielt. Wenn also in einem Jahr der Fang schlecht war (vgl. Tab. 3), waren auch seine Einkünfte aus der Vogelkoje erheblich geringer. Peters verdiente in drei Jahren insgesamt über 1.000 RM: 1876 1.285,87 RM, 1887 1.153,70 RM und 1888 1.274,00 RM. In diesen drei Jahren lag die Vogelkoje alleine zwischen 78,0 und 85,4\% seiner Einkünfte. Umgekehrt machte sie in den schlechten Jahren 1873 (529,96 RM) und 1889 (547,28 RM) nur 58,2 \% bzw. 60,0 $\%$ aus. In dem mit Gesamteinkünften von 408,58 RM noch schlechteren Jahr 1870 hatte die Vogelkoje jedoch trotzdem 84,4 \% seiner Einkünfte ausgemacht. Der Anteil der Vogelkoje an den Gesamteinkünften hing also davon ab, ob er ein schlechtes Fangergebnis durch andere Einkünfte ausgleichen konnte. 1870 war die Vogelkoje noch relativ neu, und er hatte offenbar stark auf die Einkünfte aus ihr gesetzt. Seit 1875 arbeitete er daneben mehr als landwirtschaftlicher Tagelöhner und hatte stets über 100 RM Einkünfte aus diesem Sektor, was in schlechten Fangjahren einen gewissen Ausgleich bot. 1875 machte der landwirtschaftliche Tagelohn so 23,3 \% seines Einkommens aus, 1889 19,6 \%. Hinzu kamen Einnahmen aus Wegearbeiten für die Bauernkasse.

1868, also bevor er Kojenwart wurde, verdiente er 186,45 RM oder 41,1 \% seines damaligen Einkommens beim Austernfischen. Da sein Bruder Roluf ein Austernboot hatte, war dies eine gute Möglichkeit. ${ }^{12}$ Seit 1870 verschwinden Einnahmen vom Austernfischen jedoch vollständig.

Besonders in den frühen Jahren hatte Cornelius Peters oft recht große Einnahmen im Bereich Bergelohn und Strandwesen: 1867 machten sie 61,5\% seiner Einkünfte aus, 1868 immerhin 36,8 \%, 1869 sanken sie bereits auf 21,8 \%. Man kann gut erkennen, dass diese unsicheren, von einzelnen Strandungsfällen abhängigen Einkünfte zugunsten der sichereren Einnahmen aus der Vogelkoje aufgegeben wurden. 1870 machten sie nur noch $1,7 \%$ aus, 1871 dann noch wieder 32,2 \%, 1873 13,2 \%. In den übrigen Jahren spielten sie kaum noch eine Rolle. Das Problem des - gut bezahlten - Bergelohns war, dass man sofort zur Stelle sein musste, wenn Schiffe strandeten. Wann das war und wie oft das geschah, wusste man nicht. Nicht selten geschah es jedoch gerade zwischen September und November, wenn auch die Fangzeit in der Vogelkoje war. Hier setzte Cornelius Peters klar auf die sichere (und ungefährlichere) Arbeit, nämlich die Vogelkoje. 1867 und 1868 war ein Teil seiner Einkünfte aus dem Rettungsboot erwachsen (60,00 RM bzw. 73,88 RM). Das Ausfahren im Rettungsboot war jedoch ausgesprochen gefährlich, und immer wieder kamen Retter dabei um. Diese Arbeit stand also eher einem jüngeren Mann an, als einem älteren, und es ist gut zu verstehen, dass er später die ruhigere Arbeit in der Vogelkoje vorzog.

12 Auch Roluf hat ein Tagebuch geführt, aus dem Georg Quedens Auszüge publiziert hat; Quedens (1986), S. 95-111. 
Neben diesen größeren Posten standen kleinere. So verdiente er zwischen 1868 und 1875 stets ca. 5-10\% seines Einkommens mit dem Verkauf von Fisch. Später spielte die Fischerei jedoch kaum noch ein Rolle. Man hat aus den Tagebüchern den Eindruck, dass er sie bewusst verringerte, weil Kräfte und Gesundheit nachlieBen. Meist verdiente er auch ein paar Mark aus dem Verkauf von Kaninchenfellen. Kleinere Tätigkeiten trugen jedoch zur Ökonomie der Familie bei, indem sie Lebensmittel beschafften: Fische, Fleisch, Eier, Gemüseanbau im Garten.

Im Fischgarten fing er vor allem Plattfische. Ein Fischgarten war eine Art Fischzaun, der im Wattenmeer aufgestellt wurde. Er wurde so aufgestellt, dass sich die Fische bei abfließendem Wasser darin fingen. ${ }^{13}$ Cornelius Peters leerte den Fischgarten zweimal am Tag bei Ebbe und notierte jedesmal gewissenhaft, wie viele Fische er gefangen hatte. In den Aufzeichnungen gehen die Begriffe „Schollen“ und „Butten“ durcheinander. Mitunter steht bei den Tagen „Schollen“, bei der Monatssumme jedoch „Butten“. Man wird die „Schollen“ und „Butten“ deshalb als Plattfische verstehen müssen, unabhängig von der Art. Später unterscheidet er mitunter zwischen den „Schollen“ im Fischgarten und den „Butten“ im Haff.

Die Hauptfangzeit mit dem Fischgarten war März bis Mai. Der Höhepunkt lag im April. Cornelius Peters fing große Mengen Plattfische („Schollen“). Da er sie z. T. in Drachten, in Exemplaren oder in Pfund angab, ist es schwer, die exakte Zahl zu bestimmen. Eine Dracht war offenbar die Menge, die sich auf einmal transportieren ließ. Die Anzahl der Fische ist daher nicht zuletzt von deren Größe abhängig. Da er sie selbst zusammenrechnete, kann man jedoch ungefähr den Umfang einer „Dracht“ bestimmen. Im Laufe des Monats März 1876 hatte er 997 Schollen gefangen, die er in der Monatssumme zusammen als „1 1 1/2 Dracht Schollen“ rechnet. Demnach ergaben ca. 665 Schollen 1 Dracht Schollen. Eine Dracht war jedoch ein relativer Begriff. 1872 lag eine Dracht bei etwa 654 Schollen, im April 1873 aber nur bei etwa 514 und im April 1875 bei etwa 538. Im April 1876, einem sehr guten Monat rechnete er auch schon mal 710 Schollen für nur eine halbe Dracht. Dafür bestand bei der Jahresrechnung 1875 eine Dracht aus 723 Schollen, 1876 waren es sogar 2.143(!). Hier kann es sich nur um Rechenfehler handeln - oder es handelte sich um besonders kleine Fische. Es macht für uns unter diesen Umständen keinen Sinn, die Einzelschollen in Drachten umzurechnen oder gar die Drachten in einzelne Schollen. Die Abweichung wäre zu groß. Wichtig $\mathrm{zu}$ wissen ist aber, dass die Dracht ein sehr großes Maß ist und wohl irgendwo zwischen 500 und 700 Schollen anzusetzen ist. Aus Cornelius Peters' täglichen Aufzeichnungen ergeben sich die Ergebnisse, die in Tab. 4 dargestellt sind. Die monatlichen und jährlichen Summen weichen davon zum Teil ab. Möglicherweise hängt das mit Eigenverbrauch oder Rechenfehlern zusammen.

\footnotetext{
13 Abbildungen eines Fischgartens bei Föhr finden sich in: Koehn H (1954) Die Nordfriesischen Inseln. Die Entwicklung ihrer Landschaft und die Geschichte ihres Volkstums. De Gruyter, Hamburg. Tafel 63. Vgl. auch Petersen T (1979) Rømø. Et bidrag til øens historie og beskrivelse, Åbenrå. S. 105-108 und Abb. neben S. 97.
} 
Tab. 4: Gefangene Schollen, 1867-1890.

\begin{tabular}{|c|ccc|}
\hline Jahr & Drachten & $\begin{array}{c}\text { Einzelne } \\
\text { Schollen }\end{array}$ & Pfund \\
\hline 1867 & 0 & 1 & 105 \\
1868 & 24,25 & 3409 & 127 \\
1869 & 44 & 898 & 203 \\
1870 & 0 & 2297 & 108 \\
1871 & 0 & 1352 & 10 \\
1872 & 22,25 & 2780 & 20 \\
1873 & 26 & 3402 & 0 \\
1874 & 91,83 & 583 & 0 \\
1875 & 41,5 & 3255 & 0 \\
1876 & 33,91 & 4307 & 0 \\
& & & \\
1887 & 0 & 978 & 4 \\
1888 & 1 & 0 & 0 \\
1889 & 0 & 5767 & 0 \\
1890 & 0 & 0 & 11 \\
\hline Summe & $\mathbf{2 8 4 , 7 4}$ & $\mathbf{2 9 0 2 9}$ & $\mathbf{5 8 8}$ \\
\hline
\end{tabular}

Tab. 4 zeigt, dass 1868, 1869 und die Jahre 1872 bis 1876 gute Fangjahre waren, 1874 war sogar extraordinär gut. 1867 scheint Cornelius Peters noch nicht mit dem Fischgarten gefangen zu haben. Schlecht waren die Jahre 1870, 1871 und die Jahre ab 1887. In jenen Jahren hat er den Fischgarten überhaupt nicht mehr benutzt. Die Fänge stammen, ebenso wie die in Pfundzahlen angegebenen Fänge der übrigen Jahre aus anderen Fischzügen.

Als Beifang gingen auch andere Fische in den Fischgarten: Hornhechte (385 Stück in 14 Jahren), Müller (61), Rochen (57), Dorsche (26), Steinbeißer (8), einzelne Male auch Heringe (4), Makrelen (3) oder Lachse (2). Sie gingen jedoch nicht in allen Monaten in den Fischgarten, und man kann aus den Fangmonaten Rückschlüsse auf ihr Vorkommen im Wattenmeer ziehen. So gingen Hornhechte $(81,3$ \%), Rochen (80,7 \%) und Dorsche (88,5 \%) meist im Mai in den Fischgarten, Müller dagegen im April (90,2\%).

Mitte Mai war die Schollensaison im Wattenmeer meist zuende. Jetzt musste man weiter ausfahren, um zu fischen. 1868 und 1876 ging er auf einige solche Fahrten. Wenn man mehrere Tage auf See war, blieb man danach meist zwei Tage zu Hause, bevor man wieder ausfuhr. Am 3. Juni 1868 notierte er jedoch: „Kein Fisch mehr“. 1876 ging er den 24./25. und 30./31. Mai mit seinem Bruder auf solche Fahrten. Dabei fingen sie 184 Schellfische und 128 Stieg (= 2.560) Schollen. Der Fang wurde dann unter den Teilnehmern aufgeteilt. Cornelius' Anteil waren 32 Stieg $(=640)$ Schollen und 46 Schellfische. Er selbst gab diese Touren jedoch 
auf. Erst 1889 versuchte sich sein Sohn Gerret zusammen mit Roluf wieder mit solchen Fahrten. Die erste Fahrt vom 9. bis 12. Mai brachte jedoch überhaupt nichts. Die zweite am 20./21. Mai brachte dann 88 Stieg $(=1.760)$ Schollen. Offenbar hatten sie jetzt die richtige Stelle gefunden, denn bereits am nächsten Tag fuhren sie wieder aus und kamen drei Tage später mit 200 Stieg $(=4.000)$ Schollen zurück. Damit war die Saison aber auch schon wieder zuende. Am 27. Juni fingen Roluf und Gerret aber noch einen Stör von 102 Pfund.

Neben den Plattfischen waren Aale der Hauptfang (doch wurden sie nicht im Fischgarten gefangen). In den überlieferten 14 Jahren fing Cornelius Peters insgesamt 2.259,5 Pfund Aale. Das macht einen Jahresdurchschnitt von 161,4 Pfund. Im Jahre 1870 fing er jedoch 507,5 Pfund. Die Jahre 1875, 1876, 1887 und 1888 waren mit 12 bis 33 Pfund dagegen extrem schlechte Jahre, entweder weil es keine Aale gab oder weil er nicht auf Fang ging. Seit 1874 vermied er es nämlich wegen seiner vielen Lungenentzündungen, im Winter bei kaltem oder schlechtem Wetter auf Aalfang zu gehen, so dass seine Fangergebnisse massiv einbrachen.

Tab. 5: Erlegte Kaninchen und Kaninchenfangsaison, 1867-1891.

\begin{tabular}{|cc|cc|}
\hline Jahr & Kaninchen & Monat & Kaninchen \\
\hline 1867 & 96 & Januar & 157 \\
1868 & 7 & Februar & 110,5 \\
1869 & 38 & März & 93,5 \\
1870 & 67 & April & 21 \\
1871 & 26 & Mai & 10 \\
1872 & 28 & Juni & 0 \\
1873 & 158 & Juli & 5 \\
1874 & 69,5 & August & 85 \\
1875 & 17 & September & 39 \\
1876 & 21 & Oktober & 40 \\
& & November & 51 \\
1887 & 28 & Dezember & 82 \\
\cline { 2 - 2 } 1888 & 21 & & \\
1889 & 61 & & \\
1890 & 44,5 & & \\
1891 & 12 & & \\
\cline { 1 - 2 } Summe & 694 & & \\
Durchschnitt pro Jahr & 49,6 &
\end{tabular}

Über alle Jahre fing Cornelius Peters Kaninchen. Die Wildkaninchen auf Amrum waren etwas Besonderes, da es Kaninchen ursprünglich in Schleswig-Holstein nicht gab. Kaninchen kamen nur in Spanien und Nordafrika vor. Auf Amrum 
wurden sie aber bereits im Mittelalter ausgesetzt, vermutlich in Zusammenhang mit einem Jagdhaus des dänischen Königs Waldemar II. ${ }^{14}$ Die Kaninchenjagd war auf Amrum für die Inselbevölkerung bis Mitte der 1930er Jahre frei. Weil sie im 19. Jahrhundert auf dem Festland noch kaum verbreitet waren, konnte man lebende Wildkaninchen zu einem guten Preis an Jagdbesitzer verkaufen, die sie dann in ihrem Jagdgebiet aussetzten. Im Juni 1876 verkaufte Cornelius Peters zu diesem Zweck 17 lebendige junge Kaninchen für $24 \mathrm{~m}$ an Graf Rewentlow-Criminil.

Normalerweise fing Cornelius Peters Kaninchen wegen ihres Fleisches und ihres Fells. In den 14 überlieferten Jahren erlegte er insgesamt 694 Kaninchen, das sind 49,6 Kaninchen pro Jahr. ${ }^{15}$ Die Jahre 1867 und 1873 brachten mit 96 bzw. 158 Kaninchen jedoch wesentlich höhere Resultate. Dafür lagen andere Jahre, so 1868 mit nur sieben erlegten Tieren, weit unter dem Durchschnitt. Es gab also erhebliche Schwankungen. Die Fangsaison reichte im Wesentlichen von August bis März. In den übrigen Monaten wurden nur wenige Kaninchen gefangen. Der Höhepunkt lag klar in den Wintermonaten, insbesondere im Januar und Februar. Dies hing nicht nur damit zusammen, dass in dieser Jahreszeit weniger andere Arbeiten anlagen, sondern auch dass im Winter das Fell am besten war. Cornelius Peters unterscheidet in seinen Abrechnungen deutlich zwischen Sommer- und Winterfellen. Letztere erbrachten bessere Preise. Im Winter war er jedoch zunehmend krank und vermied den Aufenthalt im Freien, was einen Teil der Schwankungen und den Rückgang seiner Fangergebnisse seit 1875 erklärt. Das extrem gute Ergebnis von 1873 ist nicht zuletzt 48 Tieren geschuldet, die er im August fing. In den letzten Jahren half ihm sein Sohn Gerret beim Kaninchenfang, was den erneuten Anstieg erklärt. Die geringe Fangtätigkeit zwischen April und Juli hängt einerseits mit anderen Arbeiten zusammen, die in dieser Zeit anstanden (Fischgarten, Ernte), andererseits ergab sich auf diese Weise eine natürliche Schonzeit, während die Kaninchen ihre Jungen hatten.

In einigen Jahren ging Cornelius Peters auch auf Seehundsjagd, doch nur unregelmäßig. 1868 schoss er 18 Seehunde, 1871 1, 18876 und 1889 13. Insgesamt erlegte er in 14 Jahren also 38 Tiere, wobei er in zwei Jahren besonders aktiv war. Auch in diesen Jahren war jedoch nicht jede Jagd von Erfolg gekrönt. Am 3. August 1868 war er bei Eidamtiefe „um Seehunden“, doch ohne Erfolg. Die Seehundsjagd war rücksichtslos und ging genau gegenläufig zur Kaninchenjagd vor sich. Die meisten Seehunde wurden im Juli geschossen, also genau in dem Monat, wo sie Junge bekommen und besonders störungsanfällig sind. ${ }^{16}$ Dies hängt damit zusammen, dass die Fischer den Seehund als Nahrungskonkurrent ansahen und bewusst ausrotten wollten. Seehunde wurden abgekocht, um das Fett zu gewinnen:

\footnotetext{
14 Quedens et. al. (1991), S. 237.

15 Da Januar und Februar 1887 in der Überlieferung fehlen und 1891 nur Januar und Februar überliefert sind, habe ich mit 14 ganzen Jahren gerechnet, auch wenn die Tabelle Daten aus 15 Jahren ausweist. - Cornelius Peters zählte junge Kaninchen als halbe. Dadurch entstehen in der Tabelle die Bruchzahlen.

${ }^{16}$ Heers KE (1999) Seehunde. Boyens, Heide. S. 27-30.
} 
„Ein Seehund abgekocht 3 Q“ (29. Juni 1871). Außerdem konnte man ihr Fell gebrauchen. Am 5. August 1868 verkaufte Cornelius Peters 14 Seehundsfelle.

Für die Amrumer war das Eiersammeln seit jeher eine wichtige Nahrungsquelle. Cornelius Peters notierte auch hier genaue Zahlen. Insgesamt hat er in den 14 dokumentierten Jahren 2.855 Eier gesammelt. Meist gibt er die Art in ihrer lokalen Bezeichnung an. Von den spezifizierten Eiern machen die „Bergenteneier“ 53,4 \% aus. „Bergente“ war die lokale Bezeichnung für die Brandente (Tadorna tadorna). Sie kommt noch heute häufig auf Amrum vor. ${ }^{17}$ Peters nahm auch andere Entengelege aus. Doch waren Spieß- und Krickenten eigentlich nur auf dem Durchzug auf Amrum und brüteten dort selten. Eher fand er Stockentengelege, die aber auch nur mit 3,5 \% zu Buch schlugen. Aber hier scheint eine Zunahme erkennbar zu sein. Erst in den 1880er Jahren tauchte die Eiderente auf der Amrumer Odde als Brutvogel auf. Wohl aufgrund des Eiersammelns blieb die Zahl der Brutpaare zunächst jedoch niedrig, ${ }^{18}$ und auch in Cornelius Peters' Tagebuch werden nur wenige Gelege aufgeführt.

15,9 \% seiner Eier machten die Silbermöweneier aus. Die Silbermöwe hat auf Amrum eine bewegte Geschichte. ${ }^{19}$ Um 1900 scheint sie völlig verschwunden gewesen zu sein, was nicht zuletzt auf das Eiersammeln zurückgeführt wurde. Ein Teil der von Cornelius Peters gesammelten Möweneier stammt jedoch auch nicht von Amrum, sondern von Sylt. Manchmal setzte er nämlich nach Hörnum auf Sylt über, wo sich eine große Silbermöwenkolonie befand. Wenn Sammler zusammen übersetzten, teilten sie danach den Ertrag. So sammelte man dort am 26. Mai 1873 134 Silbermöwen- und 8 Austernfischereier. Davon waren Cornelius' Anteil 50 Eier. Neben den Silbermöweneiern wurden auch Sturmmöweneier gesammelt, doch war ihre Zahl gering.

$21 \%$ der Eier machten „kleine Eier“ aus. Dabei dürfte es sich um die kleineren Limikolen- oder Seeschwalbeneier gehandelt haben. Die „kleinen Eier“ kamen oft vom Kniepsand. Gelegentlich spezifizierte Peters solche Eier genauer. So tauchen in seinem Tagebuch gelegentlich Austernfischer-, Kiebitz-, Rotschenkel- oder Küstenseeschwalbeneier auf. Doch war deren Zahl nicht hoch. Auch hier benutzte er in der Regel die lokalen Namen.

Cornelius Peters lebte also von den Möglichkeiten, die die reiche Natur des Wattenmeeres ihm gab: Entenfang, Fischerei, Kaninchenjagd und Eiersammeln. Durch seine Anstellung als Kojenwart in der neuerrichteten Vogelkoje Meeram bei Norddorf konnte er seine Existenz auf eine relativ sichere Grundlage stellen. Zudem arbeitete er nebenher als Tagelöhner in der Landwirtschaft und für die Gemeinde.

\footnotetext{
${ }^{17}$ Quedens G (1983) Die Vogelwelt der Insel Amrum. Buske, Hamburg. S. 43-47.

${ }^{18}$ Ebd., S. 51-57.

${ }^{19}$ Ebd., S. 90-94.
} 
Die zweite Hälfte des 19. Jahrhunderts war für Amrum eine Krisenzeit, in der viele Menschen in der Religion einen festen Halt suchten. ${ }^{20}$ Seit 1875 besuchte Cornelius Peters nach Möglichkeit jeden Sonntag die Kirche. In den Jahren 1889/90 setzte die Gemeinde auf den Tourismus und errichtete in Wittdün ein Seebad. In Norddorf wurden die Seehospize gebaut. In den folgenden Jahren entwickelte sich hier ein neuer Wirtschaftszweig, der die Existenz der lokalen Bevölkerung auf eine völlig neue Grundlage stellte. Cornelius Peters, der sich lange gegen diese Entwicklung gesträubt hatte, ${ }^{21}$ profitierte in seinen letzten Lebensjahren bereits erheblich von ihr. Sowohl er selbst als auch seine Familie fanden beim Bau der Norddorfer Seehospize reichlich Arbeit. Im Jahre 1890 verdiente er dort nicht weniger als 285,25 RM - 28,9 \% seines Einkommens. Dieser große Posten hatte zur Folge, dass die Einnahmen aus der Vogelkoje in einem relativ guten Jahr auf nur $56,9 \%$ absanken.

Der Tourismus bedeutete für Amrum wirtschaftlich eine totale Veränderung. Die Insel erlebte in den folgenden Jahren einen Boom ohnegleichen. Alles wurde jetzt dem Fremdenverkehr untergeordnet. Man bot sogar Jagdausflüge zu den Seehundsbänken an, ${ }^{22}$ und noch in den 1920er Jahren war der Entenfang in der Vogelkoje eine Touristenattraktion. Erst später entdeckte man, dass man die Ressourcen der Natur erhalten musste, um den Touristen etwas bieten zu können.

\footnotetext{
${ }^{20}$ Quedens G (1997) Kirche und Friedhöfe auf Amrum. Kirchengeschichte der St. Clemens-Kirche. Breklumer Verl., Breklum. S. 71-74, 82-84.

${ }^{21}$ Quedens (1990). S. 15-17.

22 Schlutius K (1893) Die Nordseebäder der Insel Amrum, nebst belehrendem Anhange über Wirkung und Gebrauch der Nordseebäder im Allgemeinen. Hamburg. S. 49-53.
} 
Tab. 6: Cornelius Peters' Einkommen, 1867-1890.

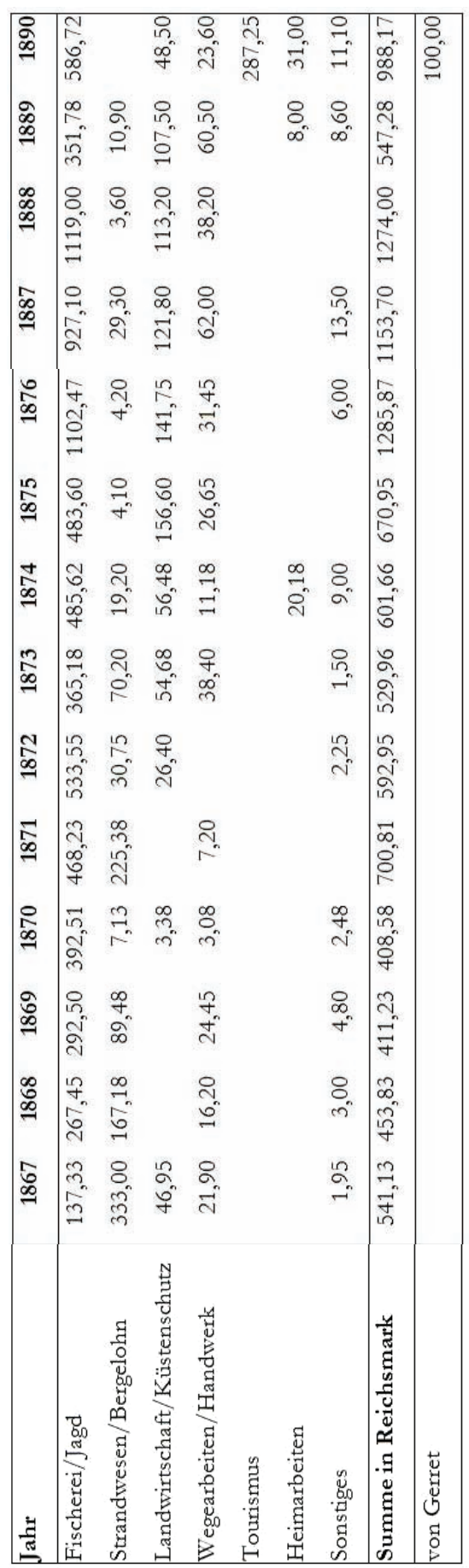


Tab. 7: Cornelius Peters' Einkommen: Anteile in Prozent am Jahreseinkommen, 1867-1890.

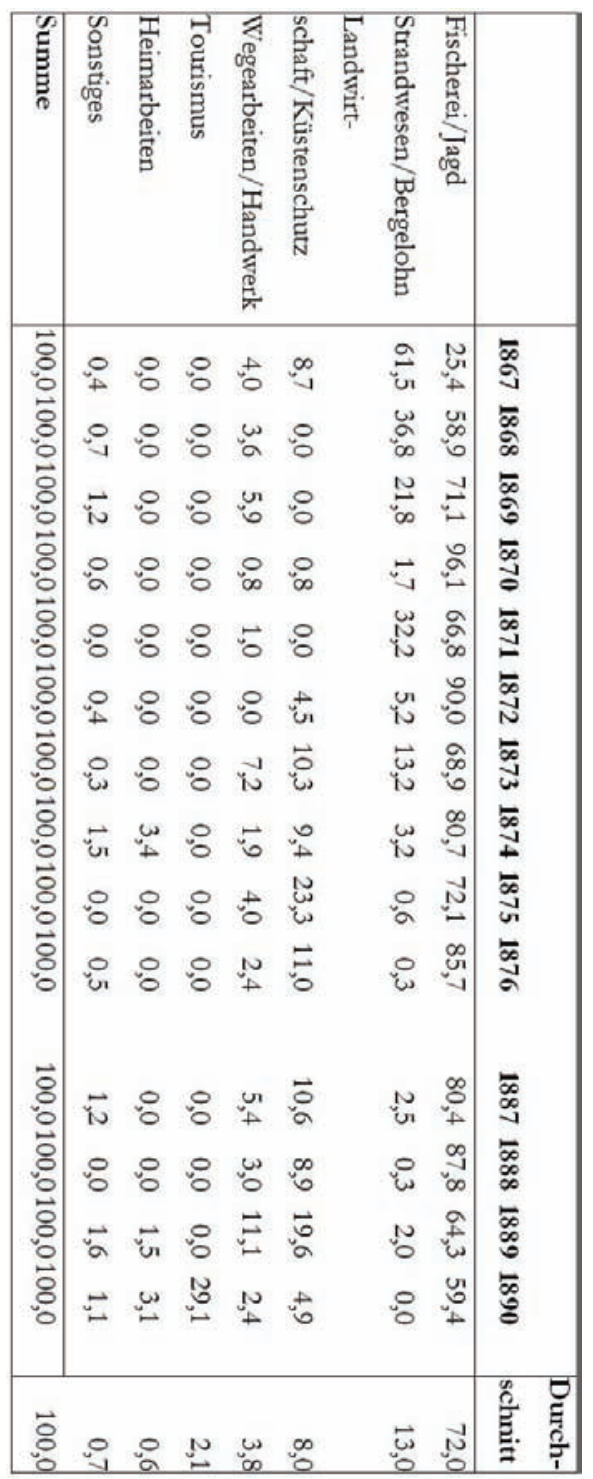




\section{Quellen und Literatur}

Heers KE (1999) Seehunde. Boyens, Heide.

Kirchenkreisarchiv Leck, Kirchenbuch St. Clemens/Amrum Nr. 1.9.

Kirchenkreisarchiv Leck, Kirchenbuch St. Clemens/Amrum Nr. 1.10.

Koehn H (1954) Die Nordfriesischen Inseln. Die Entwicklung ihrer Landschaft und die Geschichte ihres Volkstums. De Gruyter, Hamburg.

Petersen T (1979) Rømø. Et bidrag til øens historie og beskrivelse, Åbenrå.

Quedens G (1983) Die Vogelwelt der Insel Amrum. Buske, Hamburg.

Quedens G (1986) Tagebücher aus dem alten Amrum. Amrum.

Quedens G (1990) Das Seebad Amrum. Amrum.

Quedens G (1997) Kirche und Friedhöfe auf Amrum. Kirchengeschichte der St. Clemens-Kirche. Breklumer Verl., Breklum.

Quedens G, Titzck R (1991) Amrum. Landschaft - Geschichte - Natur. Amrum.

Remde F (1972) Amrum. Ein Beitrag zur Genese und Struktur einer Inselsiedlung. Diss. Univ. Münster, Münster.

Rheinheimer M (2007) Der Kojenmann. Mensch und Natur im Wattenmeer 18601900. Wachholtz, Neumünster.

Rheinheimer G (2012) Nordfriesische Seeleute in der Amsterdamer Handelsfahrt. In: Zeitschrift der Gesellschaft für Schleswig-Holsteinische Geschichte 137. S. 31-77.

Roeloffs BC (1985) Von der Seefahrt zur Landwirtschaft. Ein Beitrag zur Geschichte der Insel Föhr. Wachholtz, Neumünster.

Schlutius K (1893) Die Nordseebäder der Insel Amrum, nebst belehrendem Anhange über Wirkung und Gebrauch der Nordseebäder im Allgemeinen. Hamburg. 



\title{
Agrarinnovationen in Mittelalter und Neuzeit
}

\author{
Werner Rösener
}

\section{Einführung}

In den vergangenen Jahrhunderten wandelten sich im Zuge der Industrialisierung Wirtschaft und Gesellschaft Europas grundlegend. Im Kontext dieses Wandels veränderten sich auch die Landwirtschaft und allgemein die Lebensbedingungen im ländlichen Raum tiefgreifend. Diese Prozesse vollzogen sich sowohl im agrarischen Produktionsbereich als auch in der ländlichen Sozialstruktur, so dass einerseits die Produktivität in der Agrarwirtschaft enorm anstieg, und andererseits sich der Anteil der landwirtschaftlichen Erwerbstätigen an der Gesamtbevölkerung bedeutend verminderte. Beeindruckend sind dabei vor allem die Fortschritte in der Landwirtschaft während der vergangenen zwei Jahrhunderte. Waren um 1800 in Deutschland noch mehr als $60 \%$ der Bevölkerung im Agrarsektor beschäftigt gewesen, so sank dieser Anteil bis 1950 auf 25 \%. Im relativ kurzen Zeitraum von 1950 bis 2010 verminderte sich dieser Anteil noch rapider, nämlich auf nur noch $2 \% .^{1}$ Gleichzeitig stieg die Produktivität der Agrarwirtschaft: Waren um 1950 pro Hektar Landfläche erst 27 Doppelzentner Weizen geerntet worden, so stieg dieser Flächenertrag bis 1975 auf 44 Doppelzentner und liegt heute ungefähr bei 70 Doppelzentner pro Hektar. ${ }^{2}$ Ähnliche Produktionsfortschritte ergaben sich in der Milchwirtschaft und in der Tierzucht. Diese Fortschritte hatten allerdings auch negative Auswirkungen, wie wir noch sehen werden. Die Überproduktion wurde

\footnotetext{
1 Vgl. Henning FW (1978) Landwirtschaft und ländliche Gesellschaft in Deutschland. Band 2: 1750 bis 1976. Schöningh, Paderborn. S. 18-21; Rösener W (1993) Die Bauern in der europäischen Geschichte. Beck, München. S. 242.

2 Vgl. Henning (1978), S. 23-25.
} 
allmählich zu einem Hauptproblem der modernen Intensivlandschaft, die durch Getreideüberschüsse und durch eine hohe Milcherzeugung charakterisiert war.

Worauf beruhten diese erstaunlichen Ergebnisse in der Landwirtschaft? Welche Fortschritte sind in der Bodennutzung, in der Viehhaltung, im Pflanzenbau und in der Anwendung neuer Landbautechniken im Laufe der Zeit zu beobachten? Hat es in der vorindustriellen Zeit und vor allem während des Mittelalters keine Agrarinnovationen gegeben? In der allgemeinen Bevölkerung ist die Vorstellung von einer tausendjährigen Ruhe im Agrarbereich noch immer weit verbreitet. Sie eignet sich vorzüglich als Kulisse, vor der sich die spektakulären Ereignisse der Gegenwart abspielen. Die Vorstellung vom geschichtslosen Bauern wirkt nach, wie sie Oswald Spengler einst formulierte:

„Der Bauer ist der ewige Mensch, unabhängig von aller Kultur, die in den Städten nistet. Er geht ihr vorauf, er überdauert sie, dumpf und von Geschlecht zu Geschlecht sich fortzeugend, auf erdverbundene Berufe und Fähigkeiten beschränkt,... der Ausgang und die immer fließende Quelle des Blutes, das in den Städten die Weltgeschichte macht..." ${ }^{\text {3 }}$

Das bezeugt eine merkwürdige Enthistorisierung der Agrargeschichte, die auch heute noch oft anzutreffen ist.

\section{Agrarinnovationen im Mittelalter}

Bedeutsame Agrarinnovationen hat es nicht nur im 19. und 20. Jahrhundert gegeben, sondern auch bereits im Hochmittelalter, was oft zu wenig bedacht wird, und eine Folge der bedauerlichen Verkürzung der historischen Perspektive ist. Nur auf Grund der Fortschritte in der Agrarwirtschaft ist es im 12. und 13. Jahrhundert gelungen, die beträchtlich angestiegene Bevölkerungszahl des damaligen Europa und vor allem die Bewohner der zahlreichen neuen Städte mit genügend Nahrungsmitteln zu versorgen. Angesichts dieser erstaunlichen Leistungen der hochmittelalterlichen Landwirtschaft sprechen Historiker wie George Duby ${ }^{4}$ und Lynn White ${ }^{5}$ von einer ,agrartechnischen Revolution des Mittelalters“. In welcher Hinsicht ist dieses Urteil gerechtfertigt? Welche agrarwirtschaftlichen Fortschritte sind im Mittelalter zu verzeichnen? Ein besonderes Augenmerk ist auf die Erfolge im Ackerbau und auf den Bestand an Geräten zur Bestellung und Pflege der Felder zu richten, da die um das Dreifache vermehrte Bevölkerung damals nur durch eine gesteigerte Pflanzen- und Getreideproduktion ernährt werden konnte.

\footnotetext{
${ }^{3}$ Spengler O (1991) Der Untergang des Abendlandes. Umrisse einer Morphologie der Weltgeschichte. DVT, München. S. 113.

${ }^{4}$ Duby G (1954) La révolution agricole médiévale. In : Revue de Géographie de Lyon 29. S. 361-366. S. 361-366.

${ }_{5}^{5}$ White L (1968) Die mittelalterliche Technik und der Wandel der Gesellschaft. Moos, München. S. 39-68.
} 
Nach einer Zeit der Stagnation in Wirtschaft und Bevölkerungsentwicklung erlebten Deutschland und die benachbarten europäischen Länder vom 11. bis 13. Jahrhundert eine markante Phase des wirtschaftlichen Aufschwungs, die erst im 14. und 15. Jahrhundert von einer Krisenphase abgelöst wurde. Mit dem 11. Jahrhundert beginnt nach Marc Bloch das "Zweite Feudalzeitalter“ der mittelalterlichen Geschichte, das durch grundlegende Veränderungen in Wirtschaft, Gesellschaft und Herrschaft gekennzeichnet war. ${ }^{6}$ In agrarwirtschaftlicher Hinsicht waren die Siedlungen des frühen Mittelalters von einer auf Viehhaltung ausgerichteten einfachen Form der Feldgraswirtschaft geprägt. Die nahe bei den Einzelhöfen und Weilern gelegenen Ackerflächen waren relativ klein und dienten der Einsaat von Getreide und Gemüsepflanzen. Die Methoden der Bodenbearbeitung waren damals verhältnismäßig primitiv, und es herrschten insgesamt extensive, wenig ergiebige Formen der Bewirtschaftung vor. Die bäuerliche Bevölkerung verfügte in der Regel nur über einfache Arbeitsgeräte, die zumeist vollständig oder überwiegend aus Holz hergestellt waren, da Eisen ein kostbares Material war. Den Boden bearbeitete man mit Hacken, Eggen und einfachen Hakenpflügen unterschiedlicher Art und Qualität.

Welche agrartechnischen Veränderungen beobachtet man im Hochmittelalter bei den Arbeitsgeräten und den Anbaumethoden der Grundherren und Bauern? Bei den verbesserten Ackerbaugeräten ist an erster Stelle der Pflug zu nennen, der im Rahmen der agrartechnischen Entwicklung des Mittelalters eine vorrangige Stellung einnimmt. Obwohl es auf Grund der diffizilen Quellenlage schwer fällt, detaillierte Aussagen zur Entwicklung und Verbreitung bestimmter Pflugarten zu machen, hat die neuere Forschung aufzeigen können, dass sich der größere Beetpflug mit Rädern, Sech und schollenwendender Schar gerade im Zeitalter der hochmittelalterlichen Expansion der Agrarwirtschaft stärker durchsetzte und wesentlich zur Verbesserung der Anbautechnik beitrug. ${ }^{7}$ Er bewies seine Leistungsfähigkeit sowohl bei den älteren Anbauflächen als auch bei den schweren Böden der Niederungszonen, die neu unter den Pflug genommen wurden. Im Frühmittelalter waren die Felder noch überwiegend mit hakenförmigen Pfluggeräten bearbeitet worden, die den Ackerboden nur aufrissen und zur Einsaat unzulänglich vorbereiteten.

Obwohl der Beetpflug schon in der Spätantike sowohl in einigen römischen Provinzen als auch im Nordseeküstenbereich bekannt war, scheint er bis zum 10. Jahrhundert im okzidentalen Raum nur ganz selten vorhanden gewesen zu sein. Die archäologischen und ikonographischen Zeugnisse der früheren Zeit weisen nämlich fast ausschließlich auf Haken mit eisernen Scharen verschiedener Größe hin. Der frühmittelalterliche Bauer konnte mit seinem primitiven Pfluggerät die Felder weder in ausreichender Tiefe noch mit genügend breiter Furche beackern. Erst seit dem 11. Jahrhundert hat sich der Beetpflug offenbar in vielen Regionen

${ }^{6}$ Bloch M (1939/1940) La société féodale 1-2. Michel, Paris. S. 97.

7 Vgl. Rösener W (1985) Bauern im Mittelalter. Beck, München. S. 119-124. 
Mitteleuropas stärker durchgesetzt und den Hakenpflug schrittweise zurückgedrängt. ${ }^{8}$ Der erstaunliche Fortschritt im Ackerbau des Hochmittelalters und die erhöhte Getreideproduktion sind jedenfalls nicht zuletzt auf eine verbesserte Pflugtechnik zurückzuführen. Der schwere Beetpflug erwies sich vor allem für die nordalpinen Ebenen mit ihren regenreichen Sommern und schweren Niederungsböden von großem Vorteil. Er wurde gerade in der Expansionsphase des Hochmittelalters mit den ausgedehnten Siedlungsvorstößen zu einem wichtigen Arbeitsinstrument, das tierische Energie und technischen Fortschritt anstelle menschlicher Kraft ausnutzte. Ohne den neuen Pflug wäre es damals zweifellos schwierig gewesen, die Kultivierung der schweren Böden und der fruchtbaren Marschflächen entlang der Flüsse und Seen voranzutreiben. Sie konnten bei entsprechender Beackerung den Bauern weit höhere Erträge garantieren, als sie auf den höher gelegenen Sandböden zu erzielen waren.

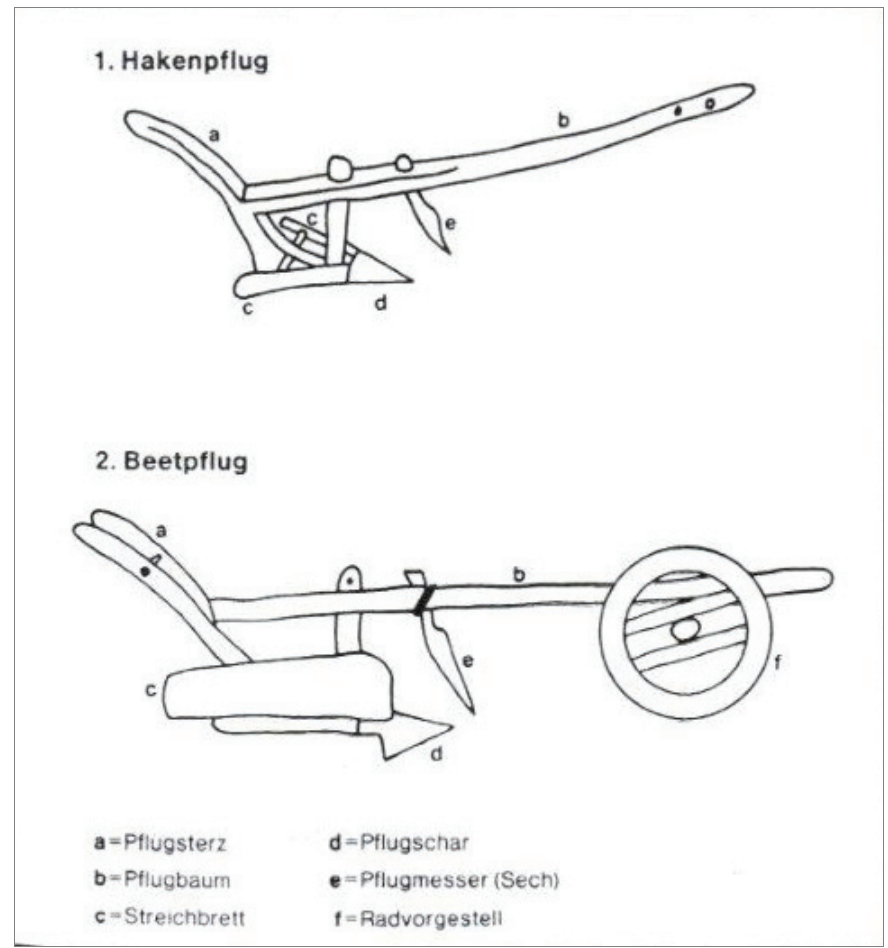

Abb. 1: Hakenpflug und Beetpflug.

8 Vgl. Abel W (1967) Geschichte der deutschen Landwirtschaft vom frühen Mittelalter bis zum 19. Jahrhundert. Ulmer, Stuttgart. S. 45-48; Bentzien U (1980) Bauernarbeit im Feudalismus. Landwirtschaftliche Arbeitsgeräte und -verfahren in Deutschland von der Mitte des ersten Jahrtausends u. Z. bis um 1800. Akadem. Verlag, Berlin. S. 60-71. 
Eine Grundvoraussetzung für die Ausbreitung des schweren Beetpfluges während des Hochmittelalters war eine verbesserte Zugkraft, da der Beetpflug eine weit höhere Zugleistung und Anspanntechnik erforderte, als dies beim leichteren Hakenpflug der Fall war. Eine verbesserte Anspannung wurde damals zum einen durch den Einsatz einer größeren Zahl von Ochsen und zum anderen durch die Ausnutzung der stärkeren Pferdekraft erreicht. Pferde, die insgesamt über eine größere Energie als Rinder verfügen, taugen aber wenig zum Pflügen, wenn sie nicht eine für sie günstige Anspannung besitzen. Obwohl Rinder auch im Hochmittelalter in vielen Gegenden weiterhin den größten Teil des Spannviehs für die Pflüge stellten, breitete sich seit dem 12. und 13. Jahrhundert vor allem im nordwesteuropäischen Raum die Verwendung von Pferden bei schweren Pflügen aus. ${ }^{9}$ Ein effektiveres Zuggeschirr für Pferde in Form von Kummeten, das die unzulänglichen Anspannvorrichtungen der älteren Zeit ablöste, tauchte bereits seit der Karolingerzeit auf und gewann besonders im Hochmittelalter wachsende Bedeutung. Beim älteren Zuggeschirr für Pferde wurde bei größerer Beanspruchung der Hals der Tiere in der Regel zu sehr eingeengt, so dass die Pferde in antiker Zeit nur als leichte Wagenpferde, fast nie aber als Zugtiere vor Pflügen oder schweren Lastkarren verwendet wurden. Neben der Entwicklung von neuen Pflügen und innovativer Anspanntechnik müssen auch andere Arbeitsgeräte wie Sense, Dreschflegel und Erntewagen erwähnt werden, die den agrartechnischen Fortschritt des Hochmittelalters beförderten. Bei den Arbeitsgeräten konstatiert man insgesamt eine deutliche Zunahme der eisernen Teile, wodurch die Geräte eine längere Haltbarkeit und größere Arbeitseffektivität erreichten.

Ein wesentlicher Faktor für den agrarwirtschaftlichen Fortschritt des Hochmittelalters war auch die Ausbreitung der Dreifelderwirtschaft. ${ }^{10}$ Die Anfänge dieses Bodennutzungssystems gehen zwar auf die Karolingerzeit zurück, doch erstreckte sich seine damalige Anwendung im Wesentlichen auf wenige Äcker. Die eigentliche Ausbreitung der Dreifelderwirtschaft in Gestalt der dörflichen Dreizelgenwirtschaft fand erst im Hochmittelalter statt, so dass dieses Feldsystem in der nachfolgenden Zeit schließlich zur vorherrschenden Form der Ackerlandnutzung in den meisten Landschaften wurde. Welche Vorteile brachte die Dreifelderwirtschaft mit sich? Welchen Einfluss hatte sie auf den Fortschritt der Agrarwirtschaft? Gegenüber den älteren Formen der Bodennutzung besaß die Dreifelderwirtschaft, die bis zum 19. Jahrhundert in den meisten Landschaften dominierte, mehrere Vorzüge, die sich in drei Punkten zusammenfassen lassen. Die neue Form des Fruchtwechsels vermehrte bei sorgfältiger Anwendung erstens die Getreideerträge beträchtlich, wobei eine geschätzte Steigerung von bis zu $50 \%$ nicht zu hoch angesetzt ist. Sie verteilte zweitens die Arbeiten des Pflügens, Säens und Erntens gleichmäßiger über das ganze Jahr und verbesserte dadurch entscheidend die bäuerliche Arbeitseffektivität. Das Feld, das die Wintereinsaat aufnehmen sollte, wurde drittens intensiver

\footnotetext{
${ }_{9}^{9}$ Bentzien (1980), S. 83-86; Rösener (1985), S. 123.

10 Duby (1962), S. 172-175; Abel (1967), S. 88-90; Rösener W (1986) Dreifelderwirtschaft. In: Lexikon des Mittelalters 3. S. 1377-1381.
} 
bewirtschaftet und gedüngt. Dies wirkte sich günstig auf den Nährstoffhaushalt der Ackerkrume aus und beugte einer zu schnellen Erschöpfung des Bodens vor.



Abb. 2: Modell eines Haufendorfs mit Gewannflur I: Wohnbereich des Dorfes mit Hofstätten und Garten II: Ackerflur mit drei Großfeldern und Wiesenland III: Allmende mit Weide- und Waldflächen

Betrachtet man die Genese der Anbausysteme im Gesamtrahmen der agrarwirtschaftlichen Entwicklung des Hochmittelalters, so stellt die Ausbreitung der Dreifelderwirtschaft zweifellos einen wichtigen Faktor für den landwirtschaftlichen Fortschritt der vorindustriellen Epoche dar. Zusammen mit der enormen Ausdehnung der Kulturflächen im Rahmen der Ostsiedlung, der Ausweitung der Getreidewirtschaft, der Verbesserung der Arbeitsgeräte und der Intensivierung des Ackerbaus schuf die Dreifelderwirtschaft die Basis für den erstaunlichen Aufschwung der Landwirtschaft während des 12. und 13. Jahrhunderts. Begünstigt wurde diese Boomphase der Agrarwirtschaft auch durch die Wärmeperiode und die Klimagunst des Hochmittelalters, wie die neuere Forschung zu Recht hervorgehoben hat. Die agrarwirtschaftliche Ertragssteigerung bildete damals die Voraussetzung für den enormen Anstieg der Bevölkerung, das Wachstum der Gesamtwirtschaft und das Aufblühen von Handel und Gewerbe. Erst auf dieser Grundla- 
ge konnten sich Stadtwirtschaft, städtische Kultur und ritterlich-höfische Lebensformen des Mittelalters voll entfalten.

\section{Agrarinnovationen in der Neuzeit}

Wenn wir uns jetzt vom Hochmittelalter aus den agrarwirtschaftlichen Entwicklungsprozessen der Neuzeit zuwenden, so sollen die Agrarinnovationen des 19. und 20. Jahrhunderts im Mittelpunkt stehen. Bevor wir aber die Themen der Mechanisierung der Landwirtschaft und der Problematik der agrarwirtschaftlichen Industrialisierung behandeln, sollen einige grundsätzliche Bemerkungen $\mathrm{zu}$ den Agrarreformen des 19. Jahrhunderts vorausgeschickt werden. Die sogenannte Bauernbefreiung gehört zweifellos zu den einschneidenden Vorgängen in der neueren deutschen Sozial- und Wirtschaftsgeschichte. Selten haben staatliche Maßnahmen so tiefgreifend in die Struktur der ländlichen Gesellschaft und Wirtschaft eingegriffen wie die Bauernbefreiung und die Agrarreformen, die in Deutschland vor allem in der ersten Hälfte des 19. Jahrhunderts sattfanden und die den Weg für eine leistungs- und marktorientierte Landwirtschaft freimachten. Die vielfältigen Maßnahmen, die im 19. Jahrhundert in Mitteleuropa durchgeführt wurden, um die alte vorindustrielle Agrarordnung aufzuheben, werden heute in der Regel mit dem Terminus „Bauernbefreiung“ bezeichnet. ${ }^{11}$ In der Sprache der Zeit nannte man dagegen die Befreiung der preußischen Gutsbauern zumeist Regulierung, die der grundherrlichen Bauern Ablösung oder Grundentlastung.

Welche agrarhistorischen Phänomene werden mit dem Begriff der Bauernbefreiung erfasst? Mit diesem Terminus werden allgemein drei Reformvorgänge charakterisiert:12 1) Ablösungen, 2) Gemeinheitsteilungen und 3) Separationen. Ablösung meint die Lösung der Bauern aus vielfältiger herrschaftlicher Abhängigkeit, bezeichnet also die Bauernbefreiung im engeren Sinne. Mit dem Ausdruck „Gemeinheitsteilung" wird die Aufteilung der bisher genossenschaftlich genutzten Flächen in der Umgebung der Dörfer, auch Allmende oder Mark genannt, an die einzelnen Nutzungsberechtigten charakterisiert. Separation oder Verkoppelung schließlich meint die Zusammenlegung des häufig in viele Parzellen zersplitterten Ackerlandes der früher kollektiv bewirtschafteten Dorfflur zu größeren Feldkomplexen, so dass diese, befreit vom Flurzwang, von einem einzelnen Betrieb unabhängig bewirtschaftet werden konnten.

Neben den skizzierten Agrarreformen wurde die moderne Agrarwirtschaft vor allem durch neue Produktionsmethoden, durch den Maschineneinsatz und durch den Prozess der Industrialisierung der Landwirtschaft stark verändert. Im Landbau wurde die traditionelle Dreifelderwirtschaft immer mehr zurückgedrängt zugunsten einer verbesserten Stufe der Bebauung. Die verbesserte Dreifelderwirtschaft wurde

\footnotetext{
11 Dipper C (1980) Die Bauernbefreiung in Deutschland 1790-1850. Kohlhammer, Stuttgart.

12 Vgl. Rösener (1993), S. 221-226; Achilles W (1993) Deutsche Agrargeschichte im Zeitalter der Reformen und der Industrialisierung. Ulmer, Stuttgart.
} 
dann ersetzt durch eine Fruchtwechselwirtschaft, so dass die Brache allmählich verschwand. Die Beseitigung des Brachfeldes und seine Bebauung mit Klee oder Hackfrüchten hatten eine beträchtliche Vergrößerung der Ackerflächen und einen bedeutenden Zuwachs der Pflanzenproduktion zur Folge. Die intensivere Bodennutzung erforderte jetzt auch eine bessere Düngung, um dem Boden genügend Ersatz für entzogene Nährstoffe zu verschaffen. Dafür stand zunächst nur der Stallmist zur Verfügung, dessen Umfang aber begrenzt war. Das Verdienst, dieses Hauptproblem der vorindustriellen Landwirtschaft im 19. Jahrhundert gelöst zu haben, kommt Justus von Liebig zu, der mit seinen Forschungen der mineralischen Düngung die Bahn freimachte. ${ }^{13}$ Bis 1914 gewann der Mineraldünger mit Kali-, Stickstoff- und Phosphatprodukten in der Landwirtschaft eine steigende Bedeutung. Gleichzeitig mit der Verbesserung der Düngung setzten Versuche zur planmäßigen Pflanzenzüchtung ein, so dass den Agrarproduzenten leistungsfähige Getreidesorten und besseres Saatgut zur Verfügung standen.

Die Agrartechnik änderte sich im frühen 20. Jahrhundert in den einzelnen Ländern Europas unterschiedlich. Während die englische Landwirtschaft eine Vorreiterrolle in der Verwendung moderner Landtechnik spielte, kamen die Mechanisierung und der Maschineneinsatz in der deutschen Landwirtschaft nur zögernd voran. Zwar stieg die Arbeitsproduktivität, d. h. die Produktion je Arbeitskraft, aber dies geschah hauptsächlich auf Grund einer erhöhten Flächenproduktivität. Pflüge, Eggen und Wagen, die in ihrer technischen Qualität allmählich verbessert wurden, waren weiterhin die wichtigsten, mit tierischer Zugkraft eingesetzten Geräte. $\mathrm{Zu}$ den arbeitssparenden Maschinen, die sich bis zum Ersten Weltkrieg in der Getreidewirtschaft ausbreiteten, gehörten vor allem Drillgeräte, Dreschmaschinen und Mähmaschinen. Da Getreide den größten Teil des Ackerlandes in Anspruch nahm, wurde offensichtlich versucht, hier in erster Linie die Arbeitsbelastung bei der Einsaat, bei der Ernte und beim Erntedrusch zu verringern. Die Hauptphase der Mechanisierung der westeuropäischen Landwirtschaft setzte nach dem Zweiten Weltkrieg ein. ${ }^{14}$ In wenigen Jahrzehnten wurde die Landwirtschaft in einem solchen Maße durch Maschinen und neue Agrartechnik verändert wie nie zuvor in der Geschichte. In Europa vollzog sich in diesem Zeitraum eine agrartechnische Revolution, die in Nordamerika bereits in der ersten Hälfte des 20. Jahrhunderts zu tiefgreifenden Veränderungen in der Landwirtschaft und zu einem wachsenden Rückgang der im Agrarsektor beschäftigten Menschen geführt hatte. Hauptansatzpunkt der zunehmenden Mechanisierung der meisten landwirtschaftlichen Arbeitsvorgänge war die verstärkte Einführung des Traktors, der nicht nur als Zugmaschine, sondern mit Hilfe der Hydraulik auch zusammen mit anderen landwirt-

\footnotetext{
${ }^{13}$ Blunck R (1946) Justus von Liebig. Die Lebensgeschichte eines Chemikers. Hammerich \& Lesser, Hamburg; Uekötter F (2010) Die Wahrheit ist auf dem Feld. Eine Wissensgeschichte der deutschen Landwirtschaft. Vandenhoeck \& Ruprecht, Göttingen. S. 146-159.

14 Vgl. Mahlerwein G (2010) Akteure im Strukturwandel. Zur Agrargeschichte des 20. Jahrhunderts. In: Geschichte in Wissenschaft und Unterricht 61. Heft 1. S. 43-55; Kluge U (2005) Agrarwirtschaft und ländliche Gesellschaft im 20. Jahrhundert. Oldenbourg, München. S. 78; Rösener (1993), S. 245248.
} 
schaftlichen Geräten als vielseitige Arbeitsmaschine eingesetzt werden konnte. Die Zahl der Traktoren, aber auch die Zahl der Mähdrescher und Melkmaschinen sind Hauptindikatoren für den Umfang des Maschinenparks und damit für den Mechanisierungsgrad in den einzelnen Regionen. Auch die Ernte der verschiedenen Feldfrüchte, der Zuckerrüben, der Kartoffeln und der Maisfrüchte, wurde zunehmend mechanisiert und mit Vollerntemaschinen bewältigt. Die Folge war eine weitgehende Ersetzung von bäuerlicher Arbeitskraft und tierischer Zugleistung durch Maschinen.

\section{Industrialisierung der Agrarwirtschaft}

Der Vorgang der Mechanisierung der Landwirtschaft in Westeuropa und in Deutschland seit dem Zweiten Weltkrieg ist eingebettet in einen größeren Prozess, der neuerdings als „Industrialisierung der Agrarwirtschaft“ charakterisiert wird. Was meint dieser Terminus? Mit diesem Begriff wird eine Entwicklung beschrieben, die durch das rasche Vordringen industrieller Produktionsformen in den Bereich der Landwirtschaft gekennzeichnet ist und deren Ergebnis die moderne Intensivlandwirtschaft darstellt. Welches sind die Hauptmerkmale dieser industrialisierten Landwirtschaft? Es sind vor allem vier parallel zueinander ablaufende und miteinander verbundene Entwicklungstendenzen, die hier hervortreten: ${ }^{15}$

agrartechnologische Innovationen

Kapitalisierung der Produktion

sektorale Konzentration der Produktion

regionale Konzentration der Agrarbetriebe.

Zweifellos stellt der agrartechnische Fortschritt mit seinen vielfältigen Innovationen die Haupttriebkraft der Industrialisierung dar. Hiermit werden insbesondere die fortschreitende Mechanisierung sowie der vermehrte Einsatz chemischer Mittel angesprochen, deren Anfänge in der Mitte des 19. Jahrhunderts liegen und bis in die Gegenwart ungebrochen weiterwirken. Die Einführung des Mineraldüngers, die Entwicklung von Traktoren und anderen Maschinen, der Einsatz von chemischen Pflanzenschutzmitteln und neuerdings die Nutzbarmachung gentechnischer Erkenntnisse in der Tier- und Pflanzenzucht markieren einige Hauptstationen dieser Entwicklung. Die Grundlage dieses agrartechnischen Fortschritts bildet immer mehr die agrarwirtschaftliche Forschung mit ihren verschiedenen Disziplinen, deren Stellenwert für die Ausbildung der modernen Intensivlandschaft nicht

\footnotetext{
15 Vgl. Windhorst HW (1989) Agrarindustrie in den USA und in der Bundesrepublik Deutschland im 19. und 20. Jahrhundert. In: T. Pierenkemper (ed) Landwirtschaft und industrielle Entwicklung. Steiner, Stuttgart. S. 237-249; Windhorst HW (1990) Industrialisierte Landwirtschaft und Agrarindustrie - was ist das? In: Alternativen in der Agrarproduktion - Ein Ausweg aus den Problemen? Vechtaer Druckerei u. Verl., Vechta. S. 11-44; Gregor HF (1982) Industrialization of U.S. Agriculture. An Interpretive Atlas. Westview Press, Boulder.
} 
hoch genug eingeschätzt werden kann. Eng verknüpft mit den agrartechnischen Innnovationen ist der starke Bedeutungsverlust der Produktionsfaktoren Boden und menschliche Arbeitskraft, die zunehmend durch den Faktor Kapital ersetzt werden. Der erhöhte Kapitalbedarf in der Landwirtschaft wird vor allem durch den Fortgang der Mechanisierung verursacht, der es möglich macht, dass immer mehr menschliche Tätigkeiten durch Maschinenkraft übernommen werden. Neben dem Einsatz von Traktoren und Vollerntemaschinen im Feldbau erlangten die finanziellen Aufwendungen im Binnenbereich der Betriebe durch Fütterungsanlagen, Melkmaschinen und sonstige technische Hilfsmittel ein zunehmendes Gewicht.

Ein weiteres Kennzeichen der Industrialisierung ist die fortschreitende sektorale Konzentration in der Landwirtschaft. In zunehmendem Maße scheiden kleine und mittlere Betriebe aus der Agrarwirtschaft aus, so dass sich die Produktion auf eine immer geringere Zahl von Großbetrieben konzentriert. Das Höfesterben geht einher mit einem Wachstum der durchschnittlichen Betriebsgröße der verbliebenen Bauernhöfe. Eine Sonderentwicklung stellt die Genese agrarindustrieller Unternehmen, sog. Agrarfabriken, dar, wie sie vornehmlich in der tierischen Veredelungswirtschaft zu beobachten ist. Hier bilden sich industriemäßige Produktionsmethoden im engeren Sinne mit neuen Formen der Betriebsorganisation. Als weiteres Merkmal der Industrialisierung ist schließlich ein Prozess von Bedeutung, der als regionale Konzentration der Agrarwirtschaft auftritt. Hierbei lässt sich beobachten, dass es in verschiedenen Produktionszweigen zu einer räumlichen Verdichtung und Verarbeitung agrarischer Güter kommt. Solche Gebiete werden häufig als „agrarische Intensivgebiete“ bezeichnet: Es sind Regionen, die auf Grund spezifischer Standortvorteile eine räumliche Konzentration der Intensivlandwirtschaft begünstigt haben. Die Weser-Ems-Region in Niedersachsen und die Region NordLimburg in den Niederlanden bilden zwei markante Beispiele agrarischer Intensivgebiete. ${ }^{16}$ Beide Regionen sind infolge ihrer hohen Spezialisierung und Intensivierung in der Tierhaltung bekannt geworden und gehören zu den führenden Zentren der Veredelungswirtschaft im nordwesteuropäischen Wirtschaftsraum - mit all ihren positiven und negativen Folgeerscheinungen.

Der Prozess der Industrialisierung und die Ausbildung agrarischer Intensivgebiete verlaufen nämlich nicht konfliktlos, sondern bringen eine Reihe ernster Folgeprobleme mit sich, welche die Zukunft der Landwirtschaft in ihrem Kern berühren. Dabei handelt es sich vor allem um zwei Problembereiche: die sozialökonomischen Auswirkungen und die ökologischen Folgen der Industrialisierung der Landwirtschaft. ${ }^{17}$ Auf der sozialökonomischen Ebene führt dieser Prozess zu einer existentiellen Gefährdung der kleinen und mittleren Bauernbetriebe. Im harten Wettbewerb der landwirtschaftlichen Betriebe und durch den zunehmenden Konkurrenzdruck der Märkte haben offenbar nur die großen kapitalstarken Betriebe eine Überlebenschance. Die Aufgabe zahlreicher Bauernhöfe hat zweifellos erheb-

16 Windhorst HW (1975) Spezialisierte Agrarwirtschaft in Südoldenburg. Eine agrargeographische Untersuchung. Schuster, Leer.

17 Vgl. Weingarten (2010), S. 6-17; Leist (2007), S. 3-10. 
liche Auswirkungen auf das dörfliche Leben und die Struktur des ländlichen Raumes. Im Mittelpunkt des allgemeinen Interesses stehen daneben vor allem die ökologischen Folgen der Industrialisierung der Landwirtschaft. Ursache dieser Konfliktzone ist die dramatische Verschärfung vieler Umweltprobleme durch die Agrarwirtschaft, die zu entsprechenden Reaktionen nicht nur in Wirtschaft und Politik, sondern auch in der breiten Öffentlichkeit geführt hat. In den Intensivgebieten mit dichter Viehhaltung konzentrieren sich die umweltrelevanten Probleme vor allem auf den Bereich der konzentrierten Viehproduktion, wozu nicht zuletzt das sog. Gülle-Problem gehört. Auf Grund der starken Viehdichte kommt es zum Anfall hoher Mengen von Gülle, so dass eine Entsorgung dieses Stoffes ohne Belastungen für die Umwelt nur schwer möglich ist. Die moderne Intensivlandwirtschaft in den Ackerbauzonen bringt weitere Umweltprobleme mit sich. Die Ausbreitung der Großflächenwirtschaft und der Monokulturen mit Mais und Raps sowie der Einsatz von Dünge- und Pflanzenschutzmitteln haben dazu geführt, dass die Intensivlandwirtschaft heute als der Hauptverursacher des rapiden Rückgangs vieler Tier- und Pflanzenarten gilt. Ein anderes Problemfeld, das gerade in der Öffentlichkeit auf breite Resonanz stößt, ist die Belastung von tierischen und pflanzlichen Nahrungsmitteln mit chemischen und biologischen Rückständen in Form von Pestiziden oder Hormonen. Die Industrialisierung der Landwirtschaft hat somit die Strukturen der agrarischen Produktion vielfältig verändert und tiefgreifende Auswirkungen gehabt.

\section{Schluss}

Wenn wir abschließend die beiden ausgewählten Epochen, das Hochmittelalter und das 19./20. Jahrhundert, hinsichtlich der Agrarinnovationen miteinander vergleichen, so konstatieren wir trotz der offensichtlichen Unterschiede auch einige Gemeinsamkeiten. Die aufgezeigten Agrarinnovationen des 12. und 13. Jahrhunderts haben in der damaligen Wirtschaft und Gesellschaft einschneidende Veränderungen und Produktionsfortschritte in der Agrarwirtschaft bewirkt, so dass während des Hochmittelalters die beträchtlich vermehrte Bevölkerung in Normaljahren mit ausreichend Nahrungsmitteln versorgt werden konnte. Im Kontext von Missernten und Naturkatastrophen kam es jedoch zu Hungerkrisen und Versorgungsengpässen; wie z. B. in der schweren Hungersnot der Jahre 1315 bis 1317. Harte Winter, verregnete Sommer und große Überschwemmungen bewirkten damals eine mehrjährige Hungerkrise, die durch ihre lange Dauer und drückende Schwere auffiel. ${ }^{18}$ Die Agrarinnovationen der zweiten Hälfte des 20. Jahrhunderts, die im Prozess der Industrialisierung der Landwirtschaft kulminierten, führten einerseits zu einer enormen Steigerung der Agrarproduktion, brachten andererseits aber schwerwiegende soziale und ökologische Folgen mit sich. Die moderne Inten-

\footnotetext{
18 Vgl. Abel W (1966) Agrarkrisen und Agrarkonjunktur. Eine Geschichte der Land- und Ernäh-
} rungswirtschaft Mitteleuropas seit dem hohen Mittelalter. Parey, Hamburg. S. 44-46; Jordan (1996). 
sivlandwirtschaft mit ihren Großbetrieben ist in vielen Ländern auf dem Vormarsch, so dass sich die Frage stellt, ob traditionelle Familienbetriebe sich in Zukunft noch behaupten können. Die ökologischen Probleme der Massentierhaltung und des ausgedehnten Maisanbaus liegen vor allem in der Umweltbelastung durch den hohen Anfall an Gülle, in der Zerstörung der Artenvielfalt und in der Belastung der Nahrungsmittel mit chemischen Rückständen. Die Industrialisierung der Landwirtschaft hat also die Strukturen der agrarischen Produktion vielfältig verändert und schwerwiegende Folgeprobleme mit sich gebracht, die noch bewältigt werden müssen.

\section{Literatur}

Abel W (1966) Agrarkrisen und Agrarkonjunktur. Eine Geschichte der Land- und Ernährungswirtschaft Mitteleuropas seit dem hohen Mittelalter. Parey, Hamburg.

Abel W (1967) Geschichte der deutschen Landwirtschaft vom frühen Mittelalter bis zum 19. Jahrhundert. Ulmer, Stuttgart.

Abel W (1974) Massenarmut und Hungerkrisen im vorindustriellen Europa. Parey, Hamburg.

Achilles W (1993) Deutsche Agrargeschichte im Zeitalter der Reformen und der Industrialisierung. Ulmer, Stuttgart.

Bentzien U (1980) Bauernarbeit im Feudalismus. Landwirtschaftliche Arbeitsgeräte und -verfahren in Deutschland von der Mitte des ersten Jahrtausends u.Z. bis um 1800. Akadem. Verlag, Berlin.

Bloch M (1939/1940) La société féodale 1-2. Michel, Paris.

Blunck R (1946) Justus von Liebig. Die Lebensgeschichte eines Chemikers. Hammerich \& Lesser, Hamburg.

Cerman M, Steffelbauer I, Tost S (ed) (2008) Agrarevolutionen. Verhältnisse in der Landwirtschaft vom Neolithikum zur Globalisierung. Innsbruck.

Dipper C (1980) Die Bauernbefreiung in Deutschland 1790-1850. Kohlhammer, Stuttgart.

Ditt K, Gudermann R, Rüße N (ed) (2001) Agrarmodernisierung und ökologische Folgen. Westfalen vom 18. bis zum 20. Jahrhundert. Schöningh, Paderborn.

Duby G (1954) La révolution agricole médiévale. In : Revue de Géographie de Lyon 29. S. 361-366.

Gregor HF (1982) Industrialization of U.S. Agriculture. An Interpretive Atlas. Westview Press, Boulder. 
Gudermann R (2000) Morastwelt und Paradies. Ökonomie und Ökologie in der Landwirtschaft am Beispiel der Meliorationen in Westfalen und Brandenburg (1830-1880). Schöningh, Paderborn.

Henning FW (1978) Landwirtschaft und ländliche Gesellschaft in Deutschland. Band 2: 1750 bis 1976. Schöningh, Paderborn.

Jordan WC (1996) Great Famine: Northern Europe in the Early Fourteenth Century. Princeton Univ. Press, Princeton.

Kluge U (2005) Agrarwirtschaft und ländliche Gesellschaft im 20. Jahrhundert. Oldenbourg, München.

Mahlerwein G (2010) Akteure im Strukturwandel. Zur Agrargeschichte des 20. Jahrhunderts. In: Geschichte in Wissenschaft und Unterricht 61. Heft 1. S. 43-55.

Pierenkemper T (ed) (1989) Landwirtschaft und industrielle Entwicklung. Zur ökonomischen Bedeutung von Bauernbefreiung, Agrarreform und Agrarrevolution. Steiner, Stuttgart.

Radkau J (2000) Natur und Macht. Eine Weltgeschichte der Umwelt. Beck, München.

Rösener W (1985) Bauern im Mittelalter. Beck, München.

Rösener W (1986) Dreifelderwirtschaft. In: Lexikon des Mittellaters 3. S. 1377 1381.

Rösener W (1992) Agrarwirtschaft, Agrarverfassung und ländliche Gesellschaft im Mittelalter. Oldenbourg, München.

Rösener W (1993) Die Bauern in der europäischen Geschichte. Beck, München.

Rösener W (2010) Das Wärmeoptimum des Hochmittelalters. Beobachtungen zur Klima- und Agrarentwicklung des Hoch- und Spätmittelalters. In: Zeitschrift für Agrargeschichte und Agrarsoziologie 58. Heft 1. S. 13-30.

Spengler O (1991) Der Untergang des Abendlandes. Umrisse einer Morphologie der Weltgeschichte. DVT, München.

Uekötter F (2010) Die Wahrheit ist auf dem Feld. Eine Wissensgeschichte der deutschen Landwirtschaft. Vandenhoeck \& Ruprecht, Göttingen.

White L (1968) Die mittelalterliche Technik und der Wandel der Gesellschaft. Moos, München.

Windhorst HW (1975) Spezialisierte Agrarwirtschaft in Südoldenburg. Eine agrargeographische Untersuchung. Schuster, Leer. 
Windhorst HW (1989) Agrarindustrie in den USA und in der Bundesrepublik Deutschland im 19. und 20. Jahrhundert. In: T. Pierenkemper (ed) Landwirtschaft und industrielle Entwicklung. Steiner, Stuttgart. S. 237-249.

Windhorst HW (1990) Industrialisierte Landwirtschaft und Agrarindustrie - was ist das? In: Alternativen in der Agrarproduktion - Ein Ausweg aus den Problemen? Vechtaer Druckerei u. Verl., Vechta. 


\title{
Weiternutzen, Reparieren, Wiederverwerten. Der „Umgang mit den Dingen“ in der Vormoderne
}

\author{
Georg Stöger
}

\section{Einleitung}

Der „Umgang mit Sachen“" , darunter soll primär das Nutzen von Alltagsgegenständen verstanden werden, hat sich im 20. Jahrhundert grundlegend verändert. In der westlichen Welt entfaltete sich seit den 1950er Jahren die Massenkonsumgesellschaft - sinkende Realpreise ließen längerfristig die Nutzungsdauer der Gegenstände absinken und die Abfallmengen ansteigen. ${ }^{2}$ Sukzessive verabschiedeten sich weite Teile der Gesellschaft von ressourcenschonenden Praktiken wie dem Weiternutzen, dem Wiederverwerten und Reparieren. Vermutlich fiel dies vielen nach den Erfahrungen des Mangels im Zweiten Weltkrieg und in den unmittelbaren Nachkriegsjahren relativ einfach, wenngleich in einzelnen Gesellschaftsschichten möglicherweise von erheblichen Persistenzen dieses „Umgangs mit Sachen“ auszugehen ist. Erst in den letzten Jahrzehnten - vor allem im Gefolge der Diskussio-

\footnotetext{
${ }^{1}$ Diese Formulierung bezieht sich auf eine Publikation aus der Volkskunde Jeggle U (1983) Vom Umgang mit Sachen. In: K. Köstlin, H. Bausinger (ed) Umgang mit Sachen. Zur Kulturgeschichte des Dinggebrauchs. 23. Deutscher Volkskundekongreß in Regensburg vom 6.-11. Oktober 1981. Univ. Regensburg, Regensburg. S. 11-25, die, partiell aus konsumkritischen Überlegungen heraus, sehr wichtige Überlegungen zur Thematik beinhaltet. Reinhold Reith (Salzburg) möchte ich für seine hilfreichen Anmerkungen zum Text herzlich danken!

2 Pfister C et al. (1995) „Das 1950er Synrom“: Zusammenfassung und Synthese. In: C. Pfister (ed) Das 1950er Syndrom. Der Weg in die Konsumgesellschaft. Haupt, Bern et al. S. 21-47; Pfister C (2010) The „1950s Syndrome“ and the Transition from a Slow-Going to a Rapid Loss of Global Sustainability. In: F. Uekötter (ed) The Turning Points of Environmental History. University of Pittsburgh Press, Pittsburgh. S. 90-118.
} 
nen um Ressourcenknappheit (man denke an das pessimistische Szenario, das die 1972 erschienene Studie Limits to Growth entwickelt hatte und die darauffolgenden Ölkrisen) und um Nachhaltigkeit - haben Versuche stattgefunden, an diese vormodernen Nutzungsformen anzuknüpfen. Gegenwärtig ist Recycling vor allem als häusliche Tätigkeit omnipräsent, und partiell postulieren - aus divergierenden Motiven (Verbraucherprotest, Konsumkritik oder Umweltschutz) - Initiativen die Verlängerung der Nutzungsdauer von Gebrauchsgegenständen, etwa über den Konsum von Gebrauchtem oder durch die Reparatur von Defektem.

Dass der Umgang mit Materiellem in der Vormoderne ${ }^{3}$ anders war, als er es gegenwärtig ist, bildet weder eine überraschende noch eine neue Feststellung. Dennoch ist diesbezüglich vieles bislang unklar; betrachten wir etwa den Bereich des Wiederverwertens: Hier stellen sich Fragen nach Ausmaß der Wiederverwertung, nach Relevanz für Wirtschaft und Gesellschaft, zudem, welche Akteure und Materialien involviert waren, wie es funktionierte, wie es sich historisch veränderte, auch wie Relevanz und damit die damit Beschäftigten wahrgenommen wurden. Dies sind Fragen, die nicht nur die Wirtschafts- und Sozialgeschichte, sondern genauso umwelt- und kulturhistorische Bereiche tangieren.

Insgesamt hielt sich das Interesse der Geschichtswissenschaft an den ,sekundären' Märkten und Zirkulationen der vorindustriellen Zeit in Grenzen. Es gibt bisher nur wenige Studien, vor allem von Seiten der Konsum- und Armutsgeschichte zum vormodernen Gebrauchtwarenhandel beziehungsweise zum Konsum von Gebrauchtem, einzelne (kleinere) Arbeiten zum Wiederverwerten und kaum bis gar nichts zum Reparieren. ${ }^{4}$ Im Folgenden soll deshalb eine Exploration dieses Themenfeldes vorgenommen werden; daher wird nur begrenzt auf archivalische Quellen zurückgegriffen und vorrangig Publiziertes verwendet - auch, um auf das Potential ,übersehener' Studien für diese Fragestellung hinzuweisen. Zunächst wird nach den Implikationen gefragt, also nach dem Warum dieses spezifischen „Umgangs mit Sachen“. Daran anschließend werden drei unterschiedliche Felder des sekundären Lebenszyklus von Gegenständen, das Weiternutzen/-geben, das Reparieren und schließlich das Wiederverwerten, in den Blick genommen, wobei nach Märkten, Akteuren und Praktiken in diesen Bereichen gefragt werden soll.

\section{Implikationen eines vormodernen „Umgangs mit Sachen“}

Nicht nur für Angehörige der Unterschichten, deren Anteil an der Bevölkerung für die vorindustrielle Zeit auf etwa die Hälfte bis zwei Drittel geschätzt werden kann,

\footnotetext{
${ }^{3}$ Darunter wird die vorindustrielle Zeit verstanden, also etwa die Periode vom 16. bis ins erste Hälfte des 19. Jahrhunderts.

${ }^{4}$ Als generelle Überblicke dazu: Enzyklopädie (2011) Bd. 13, Sp. 791-793, s. v. Trödel; Enzyklopädie (2010) Bd. 11, Sp. 58-61, s. v. Reparatur; Enzyklopädie (2011) Bd. 14, Sp. 1079-1085, s. v. Wiederverwertung.
} 
waren viele Gegenstände des täglichen Bedarfs relativ teuer. ${ }^{5}$ Das Beispiel textiler Erzeugnisse unterstreicht dies. Kleidung hatte vor der Durchsetzung der industriellen Massenproduktion, die in Kontinentaleuropa erst ab der Mitte des 19. Jahrhunderts einsetzte, einen signifikanten Wert (vgl. Tabelle 1).

Tab. 1: Preise für vormoderne Kleidung.

Quellen: für Nürnberg Groebner (1993, S. 156); für Salzburg Stöger (2011, S. 211), die Salzburger Vergleichswerte sind Archiv der Stadt Salzburg (AStS), Pezolt-Akten, No. 297 entnommen.

\begin{tabular}{|c|c|c|c|}
\hline wann/wo & was & Preis & dies entspricht ... \\
\hline $\begin{array}{c}\text { Ende 15. Jh., } \\
\text { Nürnberg }\end{array}$ & $\begin{array}{l}\text { gebrauchter } \\
\text { Männermantel }\end{array}$ & $\begin{array}{c}480 \text { Nürnberger } \\
\text { Pfennig }\end{array}$ & $\begin{array}{c}43 \text { Tagen Arbeit für } \\
\text { einen städtischen } \\
\text { Handlanger }\end{array}$ \\
\hline $\begin{array}{l}\text { um 1500, Nürn- } \\
\text { berg }\end{array}$ & $\begin{array}{l}\text { Männerhose und } \\
\text {-wams }\end{array}$ & $\begin{array}{l}2 \text { rheinische } \\
\text { Gulden }\end{array}$ & $\begin{array}{l}23 \text { Tagen Arbeit für } \\
\text { einen städtischen } \\
\text { Getreidearbeiter }\end{array}$ \\
\hline 1777, Salzburg & $\begin{array}{l}\text { gebrauchtes } \\
\text { Männerhemd }\end{array}$ & $1 \mathrm{fl} .32 \mathrm{kr}$. & $\begin{array}{l}8 \text { Tagen Arbeit für } \\
\text { einen städtischen } \\
\text { Taglöhner (1780er) }\end{array}$ \\
\hline 1784, Salzburg & $\begin{array}{l}\text { gebrauchte } \\
\text { Männerhose }\end{array}$ & $2 \mathrm{fl} .3 \mathrm{kr}$. & \\
\hline 1798, Salzburg & $\begin{array}{c}\text { „Rock“" } \\
\text { für Bauernknecht }\end{array}$ & $15 \mathrm{fl}$. & $\begin{array}{l}\text { über } 45 \text { Tagen Arbeit } \\
\text { für einen städtischen } \\
\text { Zimmerer }\end{array}$ \\
\hline 1798, Salzburg & Hose für Bauernknecht & $5 \mathrm{fl}$. & \\
\hline 1798, Salzburg & $\begin{array}{l}\text { ein Paar Schuhe für } \\
\text { Bauernknecht }\end{array}$ & $1 \mathrm{fl} .36 \mathrm{kr}$. & \\
\hline
\end{tabular}

Preisbildend war der hohe Wert des Materials, die Lohnkosten waren hingegen niedrig, was das Reparieren, das Um- und Weiterverarbeiten von Gegenständen und das Wiederverwerten von Materialien begünstigte. 6 Hinzu kam, dass viele Gegenstände wie auch Rohstoffe nur begrenzt vorhanden waren, der Rückgriff auf ,Sekundäres‘ war somit sinnvoll. Der Impetus war also ein ökonomischer, aber auch die kulturelle Dimension ist nicht zu unterschätzen: Christian Pfister hat darauf verwiesen, dass eine „Recycling-Mentalität“ als Grundzug des vormodernen

\footnotetext{
${ }^{5}$ Dies ist auf mitteleuropäische Städte bezogen - vgl. Stöger G (2011) Sekundäre Märkte? Zum Wiener und Salzburger Gebrauchtwarenhandel im 17. und 18. Jahrhundert. Verl. für Geschichte und Politik. Oldenbourg, Wien, München. S. 206.

${ }^{6}$ Reith R (2003) Recycling im späten Mittelalter und der frühen Neuzeit. Eine Materialsammlung. Frühneuzeit-Info 14. S. 47-65.
} 
Wirtschaftens anzunehmen sei. ${ }^{7}$ Die Mehrheitsbevölkerung ging sparsam mit ihrem materiellen Besitz um; dies war Konsequenz und Notwendigkeit von einer nur unzureichend abgesicherten Existenz, die sich regelmäßig mit Krisen konfrontiert sah. Infolge der geringen Einkommenselastizität, vor allem durch hohe Aufwendungen für Lebensmittel und Wohnen, wirkten Teuerungen bis weit ins 19. Jahrhundert hinein existenzbedrohend. Eine Folge war ein Repertoire an bousebold strategies, die auch den Versuch beinhalten konnten, die Lebensdauer der Gegenstände zu verlängern, nicht mehr benötigte Gegenstände weiterzugeben beziehungsweise zu verkaufen und nicht mehr Brauchbares einer anderen Nutzung zuzuführen. ${ }^{8}$ Gleichzeitig bildeten derartige Strategien auch Möglichkeiten des Wirtschaftens - somit konnte die Partizipation an sekundären Märkten und Transfers über eine bloße ökonomische Notwendigkeit hinausgehen, wie Beispiele aus mittleren und höheren sozialen Schichten zeigen. ${ }^{9}$

\section{Weiternutzen}

„Auch der hiesige Täntel- oder Trödel-Marckt ist so gar in grosser Reputation, weil man auf demselben von allen Sachen, so nur zu erdencken sind, etwa zu verkauffen antrifft [... und man kann] zuweilen daselbst etwas um einen billigen Preis kauffen, weil diejenigen Sachen, so allda feil sind, entweder aus Noth verkaufft, oder von fremden, so selbige nicht von hier mit wegnehmen können, müssen verstossen werden." ${ }^{10}$

Der Wert der Gebrauchsgegenstände (vgl. Tab. 1), auch deren (relative) Dauerhaftigkeit ermöglichte ausgeprägte Zirkulationen. Gegenstände wurden innerhalb des Haushalts, an Familienmitglieder, auch an Gesinde weitergegeben - im Falle des Gesindes war es vor allem das Überlassen von gebrauchter Kleidung, die selbst verwendet oder veräußert werden konnte. Überlassene Kleidung zählte zu den nichtmonetären Bestandteilen des Lohnes, da ihr ein konkreter Geldwert zuge-

\footnotetext{
7 Pfister (1995), S. 65.

${ }^{8}$ Fontaine L, Schlumbohm J (2000) Household Strategies for Survival: An Introduction. In: International Review of Social History 45. S. 1-17; vgl. dazu auch: Fontaine L (2011) Märkte als Chance für die Armen in der Frühen Neuzeit. In: Zeitschrift für Agrargeschichte und Agrarsoziologie 59, Nr. 2. S. 37-53; Fouquet G (2012) Sparsamkeit - ein Phänomen des Haushaltens in den Lebenswelten des Mittelalters. Harald Witthöft zum 80. Geburtstag. In: Vierteljahrschrift für Sozial- und Wirtschaftsgeschichte 99. S. 1-15.

${ }^{9}$ Welch E (2012) Sites of Consumption in Early Modern Europe. In: F. Trentmann (ed) The Oxford handbook of the history of consumption. Oxford University Press, Oxford. S. 229-250; Fouquet (2012); Stöger (2011); Ago R (2008) Using Things as Money: An Example from Later Renaissance Rome. In: L. Fontaine (ed) Alternative exchanges. Second-hand circulations from the sixteenth century to the present. Berghahn Books, New York, Oxford. S. 43-60; Storey T (2008) Prostitution and the Ciruclation of Second-hand Goods in Early Modern Rome. In: L. Fontaine (ed) Alternative exchanges. Second-hand circulations from the sixteenth century to the present. Berghahn Books, New York, Oxford. S. 61-75; Groebner V (1993) Ökonomie ohne Haus. Zum Wirtschaften armer Leute in Nürnberg am Ende des 15. Jahrhunderts. Vandenhoeck \& Ruprecht, Göttingen.

10 Küchelbecker JB (1730) Allerneueste Nachricht vom Römisch-Kayserlichen Hof [...]. Förster, Hannover. S. 706f.
} 
schrieben werden konnte - derartiges war also in der Regel kein ,Geschenk'. Auch außerhäusliche Transaktionen von Gebrauchtem waren alltäglich: Materielles fungierte als Spende an Bedürftige oder an soziale Institutionen, als direktes Zahlungsmittel, sogar im Tausch gegen Neuwaren. Gebrauchtes wurde versteigert - als Nachlass, als Folge von Verschuldung oder aus Gründen der Mobilität, war es doch sinnvoller sperrige Besitztümer am Ausgangsort zu veräußern, die Reise mit monetären Werten durchzuführen und am Zielort neue oder eben auch gebrauchte Gegenstände zu erwerben (vgl. dazu auch obiges Zitat). ${ }^{11}$

Bedeutend - obgleich sie bislang relativ unbeachtet blieb - war die Pfandleihe, also der Kredit auf Mobilien, für die vormoderne Konsumption wie Produktion. Sie bildete, sowohl in ihrer öffentlich-,karitativen“ Form (als „Pfand“- oder „Leihhäuser", in Anlehnung an die nord- und mittelitalienischen Vorbilder mitunter als montes pietatis bezeichnet) als auch als kommerzielle und private beziehungsweise informelle Pfandleihe eine häufig gewählte Form, um kurzfristigen Bargeldbedarf befriedigen zu können. Handwerker versetzten Werkzeuge und Rohmaterialien um Auftragsschwankungen auszugleichen, Privatleute belehnten entbehrliche Gegenstände, um obrigkeitliche Forderungen und andere monetäre Verpflichtungen bedienen zu können. ${ }^{12}$ Schon die aus der ,öffentlichen' Pfandleihe überlieferten Zahlen sprechen für eine starke Inanspruchnahme dieser Kreditform: Im Salzburger Leihhaus wurden in der zweiten Hälfte des 18. Jahrhundert jährlich zwischen 6.000 und 7.000 Pfänder belehnt; bei einer Einwohnerzahl von vermutlich 17.000 ist dies eine beachtliche Menge. ${ }^{13}$ Verfallene und deshalb versteigerte oder ausgelöste Pfänder zirkulierten über Händler/innen, Verpfändetes konnte zudem - was nicht legitim war und somit vorrangig die privat-informelle Leihe betraf - verliehen oder es konnte mit Versetzungsbestätigungen gehandelt werden. ${ }^{14}$

Schließlich bestand auch ein spezialisierter und omnipräsenter Handel mit Gebrauchtem. In größeren Städten, etwa in Wien, Prag, London oder Nürnberg hatten sich umfangreiche spezialisierte Wochenmärkte herausgebildet, die oftmals neben Gebrauchtwaren ebenso geringwertige Neuwaren und Dienstleistungen

\footnotetext{
11 Zander-Seidel J (1990) Textiler Hausrat. Kleidung und Haustextilien in Nürnberg von 1500-1650. Deutscher Kunstverlag, München. S. 383-397; Groebner (1993), S. 150f.; Roche D (1994) The culture of clothing. Dress and fashion in the ,ancien régime“. Cambridge University Press, Cambridge, New York. S. 88f. u. 342f.; Storey (2008); Füssel M (2009) Der Wert der Dinge. Materielle Kultur in soldatischen Selbstzeugnissen des Siebenjährigen Krieges. In: Militär und Gesellschaft in der Frühen Neuzeit 13, Nr. 1. S. 104-121.

12 Tebutt M (1984) Making ends meet. Pawnbroking and working-class credit. Methuen, London; Holbach R (1991) „Im auff Arbeit gelihen“. Zur Rolle des Kredits in der gewerblichen Produktion vom Mittelalter bis ins 16. Jahrhundert. In: M. North (ed) Kredit im spätmittelalterlichen und frühneuzeitlichen Europa. Böhlau, Köln, Wien. S. 133-158; Fontaine L (2002) Women's Economic Spheres and Credit in Pre-industrial Europe. In: B. Lemire et al. (ed) Women and Credit. Researching the Past, Refiguring the Future, Berg, Oxford, New York. S. 15-32; McCants AEC (2007) Goods at Pawn: The Overlapping Worlds of Material Possessions and Family Finance in Early Modern Amsterdam. In: Social Science History 31. S. 213-238; Stöger (2011), S. 217-232.

13 Stöger (2011), S. 228.

${ }^{14}$ Lemire B (1997) Dress, culture and commerce. The English clothing trade before the factory, 1660-1800. Macmillan, Basingstoke. S. 104-109; Fontaine (2011), S. 45-47 u. 50f.
} 
(etwa Reparaturen oder Umarbeitungen) anboten. Derartige zentrale Marktplätze, die im süddeutschen und österreichischen Raum als „Tandelmarkt" (ansonsten als „Trödel“"- oder „Grempelmarkt" ${ }^{\text {") }}$ bezeichnet wurden, bestanden vielfach bis in die zweite Hälfte des 19. Jahrhunderts. Danach scheinen sie - in einem weit geringeren Umfang - meist an die städtische Peripherie verlagert worden zu sein, was auf den Bedeutungswandel des Gebrauchten in dieser Zeit hinzudeuten vermag. ${ }^{15}$ Gebrauchtwarenhändler/innen wurden im deutschen Sprachraum zumeist als „Tandler/innen“ und „Trödler/innen“, aber auch als „Käufler/innen“ oder „Grempler/innen“ bezeichnet, in anderen Teilen Westeuropas finden sich Bezeichnungen wie old clothes men, rigattiere (bzw. rigatteri), fripiers und brocanteurs, revendeurs/revendeuses oder oudecleercopers, die auf gehandelte Gegenstände oder die Form des Handels verweisen. ${ }^{16}$ Die Händler/innen agierten in Läden, bei Ständen, auf den Plätzen und Gassen, boten aber auch ambulant Gebrauchtwaren an, was vor allem für den ländlichen Raum von Bedeutung war. Hier waren es Hausierer, die die ländliche Bevölkerung mit Neu- und Gebrauchtwaren versorgten und gleichzeitig als (partiell nicht-monetäre) Aufkäufer von Altmaterialien fungierten (vgl. dazu auch Punkt 5).

Die Angebote des spezialisierten Gebrauchtwarenhandels waren vielfältig: Gebrauchtes konnte oft zu einem geringeren Preis als Neues erworben werden, gleichzeitig war es möglich, überflüssige‘ oder entbehrliche Besitztümer relativ problemlos und in kurzer Zeit zu veräußern. Gerade der Verkauf von nachgelassenem Besitz bildete in mittleren und unteren Gesellschaftsschichten vielfach eine ökonomische Notwendigkeit. Ebenso wurde bewusst in Gebrauchsgegenstände (dazu gehörte auch Kleidung) investiert, um damit die Gefahr der Geldabwertung zu umgehen. Bei Bargeldbedarf konnten diese materiellen Investitionsgüter veräuBert oder Gegenstände - wie erwähnt - direkt als Zahlungsmittel eingesetzt werden. Die Konsumption von Gebrauchtem ist als überaus flexibel einzuschätzen und bildete vor allem für untere soziale Schichten eine basale Notwendigkeit. Gleichzeitig bot sie eine Möglichkeit des Wirtschaftens (Ausgabenreduktion), zudem konnte man über gebrauchte Gegenstände, was vor allem Kleidung betraf, höhere soziale Schichten imitieren, sich auch modisch oder ,respektabel' kleiden. ${ }^{17}$ Von Bedeutung waren auch illegitime und illegale Praktiken des Austausches und des Handels, beispielsweise mit gestohlenen oder unterschlagenen Gegenständen, was sehr oft Kleidung betraf. Dennoch kann - anders als von zeitgenössischen Beobachtern mitunter suggeriert - nicht von einer überdurchschnittlichen Partizi-

\footnotetext{
${ }^{15}$ Enzyklopädie (2011), Bd. 13, Sp. 791-793, s.v. Trödel; Roche (1994), S. 270 u. $335 f$.

16 Stöger (2011), S. 23-25.

${ }^{17}$ Allerston P (2000) Clothing and early modern Venetian society. In: Continuity and Change 15, Nr. 3. S. 367-390; Stobart J (2006) Clothes, Cabinets and Carriages: Second-hand Dealing in Eighteenth-century England. In: B. Blondé et al. (ed) Buyers and Sellers. Retail Circuits and Practices in Medieval and Early Modern Europe. Brepols, Turnhout. S. 225-244; Storey (2008); Van Damme I, Vermoesen R (2009) Second-hand Consumption as a Way of Life: Public Auctions in the Surroundings of Alost in the Late Eighteenth Century. In: Continuity and Change 24, Nr. 2. S. 275-305. Hier S. 291f.; Stöger (2011), S. 205-217.
} 
pation des ,allgemeinen' Gebrauchtwarenhandels an derartigen Transfers ausgegangen werden. ${ }^{18}$

Die Omnipräsenz der Zirkulation von Gebrauchtem bedingte die Ausprägung spezifischer Märkte. Händler/innen von Gebrauchtem waren teilweise in Zünften organisiert, belegt ist dies für einzelne niederländische, nord- und mittelitalienische, deutsche und österreichische Städte. Zünftische Akteure blieben aber in der Regel eine Minderheit - es gab eine Vielzahl anderer Berechtigungen zum Handel mit Gebrauchtem, die durch örtliche Obrigkeiten vergeben wurden. Mancherorts erhielten auch Soldaten (und deren Familienangehörige) durch die Militärbehörden Handelsberechtigungen oder derartige Tätigkeiten wurden toleriert. Zudem ist der informelle Handel mit Gebrauchtem, also die Tätigkeit unberechtigter oder nur teilweise berechtigter Händler/innen, als sehr umfangreich einzuschätzen: Der Zugang zum Gebrauchtwarenhandel war, da die Nachfrage ausgeprägt war und nur wenige bis gar keine Investitionen nötig waren (es konnten zunächst ja eigene Besitztümer oder Gegenstände für andere veräußert werden), relativ einfach. Zudem war der städtische Gebrauchtwarenhandel bis ins beginnende 19. Jahrhundert relativ wenig reglementiert, in kleineren Städten und am Land partiell überhaupt nicht. In sozioökonomischer Hinsicht ist dementsprechend bei den Händlern/innen eine signifikante Bandbreite festzustellen, die mit der Situation in den vormodernen Massenhandwerken (besonders der Schneider und Schuster) vergleichbar scheint: Es gab wenige vermögende Akteure, die einen upper market bedienten und viele formelle wie informelle Händler/innen, die den labouring poor zuzurechnen sind. ${ }^{19}$

Besonders bedeutend war der Gebrauchtwarenhandel als Erwerbsmöglichkeit für Frauen und für von anderen Tätigkeiten Exkludierte, etwa Angehörige ethnischer oder religiöser Minoritäten. Hier bestanden Erwerbsmöglichkeiten, die diesen Akteuren teilweise auch von Seiten der Obrigkeiten zugestanden wurden. Frauen scheinen weite Teile des Gebrauchtwarenhandels dominiert zu haben, wenngleich - vor allem in mittleren und höheren Segmenten - von einem Verdrängungsprozess durch männliche Akteure während der Frühen Neuzeit auszugehen ist; informelle Handelsbereiche blieben jedoch von Frauen geprägt. Analog dazu fungierte der Gebrauchtwarenhandel als Verdienstmöglichkeit für Jüdinnen und Juden, da diese bis ins ausgehende 18. Jahrhundert (partiell bis ins beginnende 19.) von zahlreichen Erwerbstätigkeiten ausgeschlossen und weitgehend auf Kleinhandel, auf Geld- und Pfandleihe beschränkt waren. Über die Leihtätigkeiten bestand eine enge Verbindung zum Handel mit Gebrauchtem, zudem bildete dieser

\footnotetext{
${ }^{18}$ Stöger (2011), S. 145-153; Lemire (1997), S. 122-136; Roche (1994), S. 337-344.

19 Roche (1994), S. 352f.; Deceulaer H (2008) Second-hand Dealers in the Early Modern Low Countries: Institutions, Markets and Practices. In: L. Fontaine (ed) Alternative exchanges. Second-hand circulations from the sixteenth century to the present. Berghahn Books, New York, Oxford. S. 13-42; Van Damme I (2010) Second-Hand Dealing in Bruges and the Rise of an ,Antiquarian Culture', c. 1750-1870. In: J. Stobart, I. Van Damme (ed) Modernity and the Second-Hand Trade European Consumption Cultures and Practices, 1700-1900. Palgrave Macmillan, Houndmills. S. 73-92; Stöger (2011), S. 169-204.
} 
einen Bereich, der nur schwach reglementiert und zu dem der Zugang relativ einfach war (vgl. oben). Somit prägte sich in einzelnen westeuropäischen Städten mit einem größeren jüdischen Bevölkerungsanteil, etwa in Prag, Venedig, Amsterdam und London, ein spezifisch ,jüdischer' (oder ein zumindest als solcher gedeuteter) Gebrauchtwarenhandel aus. Auf dem Land waren ambulant ausgeübte jüdische Kleinhandelstätigkeiten oftmals mit Gebrauchtem verbunden, da Neuwaren gegen Gebrauchtes oder Altmaterialien getauscht wurden. ${ }^{20}$

Es ist schwer abschätzbar, wie umfangreich der vormoderne Gebrauchtwarenhandel war. Hier bestehen - wie auch für die meisten Bereiche des vormodernen Gewerbes und der Konsumption - erhebliche Quantifizierungsprobleme: Das Handelsvolumen kann nur punktuell festgestellt werden, schon die Anzahl der in diesen Bereichen tätigen Akteure zu schätzen, ist sehr schwierig. Zeitgenössische Gewerbezählungen sind oft unvollständig, Steuerlisten erfassen - falls sie überhaupt greifbar sind - nur einzelne Akteursgruppen, auch der umfangreiche informelle Sektor erschwert eine Quantifizierung; die angeführten Zahlen (vgl. Tab. 2) sind also als Mindestwerte zu erachten. Hinzu kommt, dass der formelle Zugang zum Markt von den Obrigkeiten vielfach auch künstlich beschränkt wurde.

Die kontemporären Wahrnehmungen des Gebrauchtwarenhandels sind als ambivalent zu erachten: Es finden sich positive Einschätzungen, die Nutzen und Zweckmäßigkeit betonen, spottende Beschreibungen, die die Ärmlichkeit, die Unreinheit und dubiose Praktiken der Händler/innen zum Sujet machten, bis hin zu obrigkeitlichen Bedenken, die Berührungspunkte mit kriminellen Bereichen und eine mögliche Begünstigung der Verbreitung von Infektionskrankheiten sahen. ${ }^{21}$ Die Rezeption des Gebrauchtwarenhandels in der zeitgenössischen Populärkultur war jedenfalls breit - Protagonisten/innen finden sich in literarischen Werken ${ }^{22}$ und in bildlichen Darstellungen, vor allem in den seit dem 17. Jahrhundert weit verbreiteten sogenannten „Kaufruf“-Drucken, die ambulant ausgeübte Gewerbe thematisierten. ${ }^{23} \mathrm{Ab}$ dem beginnenden 19. Jahrhunderts zeichnet sich ein Wandel dieser eher vielschichtigen Wahrnehmungen ab - zunehmend wurde der Gebrauchtwarenhandel zum Synonym für Armut und als Anachronismus gedeutet. In den 1840er Jahren beschrieb Adalbert Stifter den ,großen“ Wiener Tandelmarkt, der damals noch auf der zwischen der inneren Stadt und den Vorstädten gelegenen

\footnotetext{
20 Heilig B (1931) Die Vorläufer der mährischen Konfektionsindustrie in ihrem Kampf mit den Zünften. In: Jahrbuch der Gesellschaft für Geschichte der Juden in der Čechoslovakischen Republik 3. S. 307-448; Lemire (1997), S. 75-79 u. 97-100; Stöger (2011), S. 175-180; Shesgreen S (2002) Images of the Outcast. The Urban Poor in the Cries of London. Manchester University Press, Manchester. S. 168.

${ }^{21}$ Roche (1994), S. 138; Stöger (2011), S. 233-244.

22 Beispielsweise in: Anonymus (1494) Ein liepliche histori und warheit von vir kaufmendern. S.n., s.l. (Nürnberg) - Onlinefaksimile: http://daten.digitale-sammlungen.de/ db/0002/bsb00027693 /images; E. I. C. P. N. (1682) Die Kluge Trödel-Frau. Worinnen enthalten Allerhand Listige KunstGriffe. Dadurch der Eigennutz heutiges Tages von Jederman gesuchet wird. S.n., s.l.; Reuter C (1961) Graf Ehrenfried. Abdruck der Erstausgabe von 1700. Hrsg. von W. Hecht. Niemeyer, Tübingen.

23 Beall KF (1975) Kaufrufe und Strassenhändler. Cries and itinerant trades. Eine Bibliographie. A bibliography. Hauswedell, Hamburg; Sheasgreen (2002).
} 
Verteidigungsfläche, dem Glacis, gelegen war, bereits als altertümliches Relikt. ${ }^{24}$ Dennoch scheint der fundamentale Bedeutungswandel des Gebrauchten erst im Verlauf der Industrialisierung, also in der zweiten Hälfte des 19. Jahrhunderts, einzusetzen: Die Massenproduktion (und damit sinkende Preise für Neuwaren), auch Hygienisierungsdiskurse definierten die Beziehungen zwischen dem Neuen und dem Alten neu und machten den Gebrauchtwarenhandel zu einer ökonomischen Randerscheinung, wenngleich Gebrauchtes für einzelne Bevölkerungsgruppen bis ins 20. Jahrhundert Bestandteil des alltäglichen Wirtschaftens blieb. ${ }^{25}$

Tab. 2: Anzahl formeller Gebrauchtwarenhändler/innen in ausgewählten europäischen Städten, 18. Jahrhundert.

Quellen: Stöger (2011), S. 157; Roche (1994), S. 347; Deceulaer (2008), S. 27.

\begin{tabular}{|c|c|c|c|}
\hline wann/wo & absolute Zahl & $\begin{array}{c}\text { pro } 1.000 \\
\text { Einwohner }\end{array}$ & Anmerkung \\
\hline 1736, Wien & 188 & 1,18 & \\
\hline 1771, Wien & 250 & 1,30 & \\
\hline 1812, Wien & 536 & 2,25 & \\
\hline $1720 \mathrm{er}$, Paris & ca. 700 & 1,17 & $\begin{array}{l}\text { ohne revendeurs } \\
\text { bzw. revendeuses }\end{array}$ \\
\hline $\begin{array}{l}\text { Mitte 18. Jh., } \\
\text { Antwerpen }\end{array}$ & & 0,9 & \\
\hline $\begin{array}{l}\text { Mitte 18. Jh., } \\
\text { Brüssel }\end{array}$ & & 2,8 & \\
\hline $\begin{array}{l}\text { Mitte 18. Jh., } \\
\text { Gent }\end{array}$ & & 5,1 & \\
\hline
\end{tabular}

${ }^{24}$ Stifter A (1844) Der Tandelmarkt. In: Wien und die Wiener in Bildern aus dem Leben. [...]. Heckenast, Pesth. S. 227-241.

25 Van Damme (2010); Charpy M (2008) The Scope and Structure of the Nineteenth-century Secondhand Trade in the Parisian Clothes Market. In: L. Fontaine (ed) Alternative exchanges. Second-hand circulations from the sixteenth century to the present. Berghahn Books, New York u. Oxford. S. 127151; vgl. Mayhew H (1861) London Labour and the London poor. [...]. Bd. 2. Griffin u. Bohn, London. S. 5-47. 


\section{Reparieren}

„Wenn man so was kauft, wo es nach der Hand etwas auszubessern giebt, muß mann immer nach dem gleich eine Ehle für sich besonders nehmen, weil man den näm[lichen] Zeug gewis nicht mehr haben kann, und wegen einer ausbesserung der ganze Schlafrock nicht kann weggeworffen oder ein anderer Harlekinfleck hinauf gesetzt werden." 26

Leopold Mozarts Aufforderung an seine Tochter widerspiegelt vermutlich weniger eine übertriebene Form von Sparsamkeit, sondern übliche zeitgenössische Haushaltsstrategien: Das Reparieren, wie auch andere sekundäre Nutzungs- und Tätigkeitsformen, bildete bis ins 20. Jahrhundert einen wesentlichen Bestandteil einer „Ökonomie der Notbehelfe“"27, wie sie Olwen Hufton treffend genannt hat und es übertraf dessen heutige Bedeutung sicherlich erheblich. Als Verlängerung der Nutzungsdauer war das Ausbessern von Gebrauchsgegenständen sinnvoll, da die Mehrheit der vormodernen Bevölkerung in einer Knappheitsökonomie wirtschaftete. Da viele Gebrauchsgegenstände (wie erwähnt) teuer waren, war der Impetus zu Reparieren hoch und das Ausbessern zählte zu einer alltäglichen Haushaltsstrategie. Dabei waren Ausbesserungsarbeiten nicht nur auf die städtischen labouring poor beschränkt, auch vermögendere Haushalte nahmen regelmäßig Reparaturdienste in Anspruch, wie etwa Haushaltsbücher und Korrespondenzen zeigen. ${ }^{28}$ Genauso relevant war das Instandsetzen beziehungsweise Instandhalten bei öffentlichen und semi-öffentlichen Institutionen, also bei Waisenhäusern, Spitälern oder dem Militär.

Das Reparieren war teilweise mit Umarbeitungen verbunden: Bei Kleidung etwa bildete dies eine Notwendigkeit (zum Ersetzen schadhafter Stellen), es ermöglichte mitunter auch Anpassungen an wechselnde Moden oder ein ,Befreien“ aus ursprünglichen Kontexten - wenn beispielsweise Livrees, also die Kleidung von Dienstboten, oder Uniformen einer ,sekundären‘ zivilen Nutzung zugeführt werden sollten. ${ }^{29}$ Die Reparaturfähigkeit war natürlich auch bei vormodernen Gebrauchsgegenständen begrenzt. Die Entscheidung, wann etwas zu reparieren beziehungsweise nicht mehr reparaturfähig war, variierte offenbar auch nach finanziellen Möglichkeiten, in Bezug auf Kleidung vermutlich zudem wesentlich nach Kriterien der Repräsentativität. ${ }^{30}$ Nicht mehr zu Reparierendes konnte - je

\footnotetext{
26 Brief Leopold Mozarts an seine Tochter, 8. Dezember 1786, in: Internationale Stiftung Mozarteum Salzburg (2005) Mozart. Briefe und Aufzeichnungen. Gesamtausgabe. Bd. 3. Bärenreiter, Kassel et al. S. 618.

$27 \mathrm{Im}$ Original economy of makeshifts - Hufton O (1974) The Poor of Eighteenth-century France 1750-1789. Clarendon Press, Oxford. S. 69-127.

28 Stöger (2011), S. 21f.; Pennell S (2012) Material culture in seventeenth-century ,Britain“. The matter of domestic consumption. In: Trentmann F (ed) The Oxford handbook of the history of consumption. Oxford University Press, Oxford. S. 64-84. Hier S. 78-82.

${ }^{29}$ Heilig (1931), S. 414 f.

30 Internationale Stiftung Mozarteum Salzburg (2005), S. 85.
} 
nach Zustand und Material - anderen Verwendungen zugeführt werden; am Ende der sekundären Nutzung stand die Verwertung als ,Rohstoff‘ (vgl. dazu Punkt 5).

Tab. 3: Verhältnis des Gesamtpreis von Kleidung zu den Arbeitskosten und Kosten für textile Reparaturen, Salzburg und Proßnitz (Mähren, heute Prostějov) 1770er-90er Jahre. Quellen: Státní okresní archiv Prostějov, archiv města Prostějov, Karton 4/Fasz. 30 (Stará registratura. Akta hospodař. úřadu etc. 1786); Salzburger Intelligenzblatt, 4. August 1798, S. 487; AStS, Handschriften PA 1.172 (Haushaltungs-Unkosten Buch, Bd. 3. 1760-1771), unpag.

\begin{tabular}{|c|c|c|c|c|}
\hline wann/wo & was & Gesamtpreis & Arbeitslohn & $\begin{array}{c}\text { Anteil des } \\
\text { Arbeitslohnes } \\
\text { am Gesamtpreis }\end{array}$ \\
\hline $\begin{array}{l}\text { 1786, } \\
\text { Proßnitz }\end{array}$ & $\begin{array}{l}\text { Anfertigung von fünf } \\
\text { Männermänteln und } \\
\text { Hemden }\end{array}$ & $25 \mathrm{fl} .30 \mathrm{kr}$. & $3 \mathrm{fl}$. & $11 \%$ \\
\hline $\begin{array}{l}1798 \\
\text { Salzburg }\end{array}$ & $\begin{array}{l}\text { Anfertigung eines } \\
\text { Frauen-,,Rocks“" }\end{array}$ & $10 \mathrm{fl}$. & $12 \mathrm{kr}$. & $2 \%$ \\
\hline $\begin{array}{l}1770 \\
\text { Salzburg }\end{array}$ & $\begin{array}{l}\text { „für ein Rokh } \\
\text { außgebeßert" }\end{array}$ & - & $12 \mathrm{kr}$. & - \\
\hline $\begin{array}{l}1770 \\
\text { Salzburg }\end{array}$ & $\begin{array}{l}\text { „für } 2 \text { Hoßen zu flicken } \\
\text { und neu zu fiedern“" }\end{array}$ & - & $29 \mathrm{kr}$. & - \\
\hline
\end{tabular}

Reparaturen wurden partiell im häuslichen Rahmen durchgeführt (auch durch Gesinde), das Instandsetzen hatte aber auch ein erhebliches außerhäusliches Beschäftigungspotential, denn bei komplexeren Ausbesserungen griff man vermutlich auf spezialisierte Dienstleister zurück. ${ }^{31}$ Dies wurde durch niedrige Arbeitskosten begünstigt (vgl. Tabelle 3), auch - etwa bei Kleidung - durch die Gefahr teures Material durch unsachgemäße Reparatur zu entwerten.

Reparaturen wurden durch Neuproduzenten im Handwerk ausgeführt; partiell kam es zur Herausbildung eigener Reparaturberufe: etwa der Schuhflicker (diese wurden auch als „Altmacher“, „Lepper“, „Refler“ oder „Altreissen“ bezeichnet), Flickschneider oder Kesselflicker. In manchen Städten bildeten Reparaturberufe sogar eigene Korporationen, dies war aber eher in Städten mit ausdifferenziertem Gewerbe (zum Beispiel in Nürnberg) der Fall. ${ }^{32}$ Die Darstellung eines Altmachers (also eines Schuhflickers) hat es sogar in Christoph Weigels Ständebuch, das in den 1690er Jahren entstanden ist, gebracht (Abb. 1). Es finden sich typische Schusterwerkzeuge, Leder zum Ausbessern, ein Holzbottich zum Wässern des Leders und

\footnotetext{
${ }^{31}$ Styles J (2007) The Dress of the People. Everyday Fashion in Eighteenth-century England. Yale University Press, New Haven, London. S. 73-75; Coffin JG (1994) Gender and the Guild Order: The Garment Trades in Eighteenth-Century Paris. In: The Journal of Economic History 54. S. 768-793. Hier S. 771.

32 Reith (2003), S. $50 f$.
} 
einige zu reparierende Schuhe bzw. Schuhfragmente. Die für diesen Stich und für viele andere Abbildungen des Weigelschen Ständebuches überlieferten Vorzeichnungen, die Georg Christoph Eimmart (1638-1705) angefertigt hat, deuten darauf hin, dass es sich bei dieser Darstellung um eine vermutlich relativ realitätsnahe handelt, die in Anlehnung daran entstanden ist, was Eimmart in Nürnberger Werkstätten zu Gesicht bekam. ${ }^{33}$

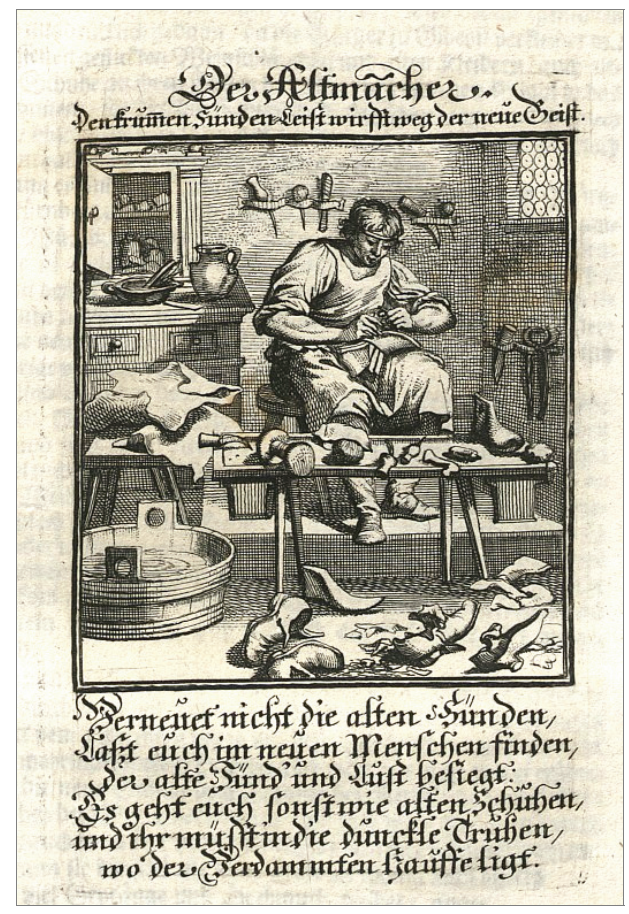

Abb. 1: Der Altmacher, Stich von Christoph Weigel, 1690er Jahre (aus: Weigel [1698], nach 650).

Wenn man die regelmäßigen Konflikte um das Ausbessern und Umarbeiten betrachtet, wird deutlich, dass die Relevanz des Reparierens im Gewerbe vor allem für ärmere Handwerker hoch war. Aus heutiger Perspektive verwundert es, dass sogar kleinere Ausbesserungsarbeiten Konflikte hervorrufen konnten, etwa, als zur Mitte des 18. Jahrhunderts die Wiener Schlosserzunft gegen einen Alteisenhändler auftrat, der hölzerne Schraubstöcke mit den von ihm verkauften Beschlägen repa-

33 Vgl. Reith R (1998) Praxis der Arbeit. Überlegungen zur Rekonstruktion von Arbeitsprozessen in der handwerklichen Produktion. In: R. Reith (ed) Praxis der Arbeit. Campus, Frankfurt a. M., New York. S. 11-54. Hier S. 21; die restlichen Stiche basieren auf Entwürfen des Niederländers Caspar Luyken. 
riert hatte. ${ }^{34}$ Zahlreiche Akteure waren außerhalb des Zunftsystems tätig: Soldaten und Soldatenhandwerker, „Störer“, also meist Gesellen, die außerhalb von Meisterhaushalten arbeiteten ${ }^{35}$, aber auch Gebrauchtwarenhändler/innen. Hier bestand ein Konnex über die (mitunter notwendige) Aufbereitung von angekauften Gebrauchtwaren, zudem hatten zahlreiche Händler eine handwerkliche Ausbildung durchlaufen. ${ }^{36}$ Partiell zeichnet sich bei einigen Reparaturdienstleistern eine Verbindung zu anderen Tätigkeiten ab, etwa zum (Klein-)Handel mit Gebrauchtem oder Neuwaren beziehungsweise zu anderen Dienstleistungen und Sammeltätigkeiten. Kesselflicker sammelten vielfach Altmetall und handelten damit ${ }^{37}$, Schuhflicker boten auch alte Schuhe zum Verkauf an. ${ }^{38}$

Einerseits wurde in Werkstätten und Unterkünften repariert, von Bedeutung waren aber genauso ambulante Formen des Reparierens, in der Stadt, wie auf dem Land. Auf abgelegenen Bauernhöfen wurde das Reparieren in Form der „Stör“ im ostalpinen Raum bis ins 20. Jahrhundert praktiziert. ${ }^{39}$ Aber auch in der Stadt waren - wie verschiedene bildliche und literarische Quellen nahe legen - Reparaturtätigkeiten, die außerhalb von Werkstätten, auf den Straßen und Gassen, den Plätzen oder in Durchgängen ausgeübt wurden, anzutreffen. ${ }^{40}$ Vor allem arme und/oder informelle Akteure wurden in dieser Form tätig. Da die Reparaturen ein breites Spektrum umfassten, sind die dazu erforderlichen Fähigkeiten und Werkzeuge als sehr unterschiedlich zu erachten: Alltägliche' Ausbesserungsarbeiten konnten mit wenig Werkzeug bewerkstelligt werden, komplexere Tätigkeiten erforderten hingegen eine erhebliche Expertise beziehungsweise eine umfangreichere Geräteausstattung. Oft wurden Gesellen mit Flickarbeiten beschäftigt, die partiell als Zuverdienst fungieren konnten. ${ }^{41}$ In Handwerkerhaushalten, die Reparaturen durchführten, waren auch Frauen und Kinder in vorbereitende Arbeiten eingebunden. ${ }^{42}$ In sozioökonomischer Hinsicht können Reparaturdienstleister insgesamt vermutlich

\footnotetext{
34 Stöger (2011), S. 117.

${ }^{35}$ Enzyklopädie (2010), Bd. 12, Sp. 1050-1052, s. v. Störer.

36 Stöger (2011), S. 40f. u. 117 f.

37 Scheffknecht W (2001) Fremde Wanderkrämer und Keßler in der Grafschaft Hohenems und im Reichshof Lustenau. In: M. Häberlein, M. Zürn (ed) Minderheiten, Obrigkeit und Gesellschaft in der Frühen Neuzeit. Integrations- und Abgrenzungsprozesse im süddeutschen Raum. Scripta Mercaturae, St. Katharinen. S. 233-267. Hier S. 238f.

38 Weigel C (1698) Abbildung Der Gemein-Nützlichen Haupt-Stände [...]. s. n., Regensburg. S. 651.

39 Glass C (1983), Reparierendes Handwerk. In: Ludwig-Uhland-Institut f. Emp. Kulturwiss. der Univ. Tübingen, Württemberg. Landesmuseum Stuttgart (ed) Flick-Werk. Reparieren und Umnutzen in der Alltagskultur. Begleitheft zur Ausstellung im Württembergischen Landesmuseum Stuttgart vom 15. Oktober bis 15. Dezember 1983. Württembergisches Landesmuseum, Stuttgart. S. 35-42. Hier S. 35; Reith (2003), S. 57; Scheffknecht (2001), S. 237-239 u. 254-256; Hornschuch F (1930) Aufbau und Geschichte der interterritorialen Kesslerkreise in Deutschland. Kohlhammer, Stuttgart. S. $99 \mathrm{f}$.

40 Shesgreen (2002), S. 14f., 43, 52 u. 157; Beall (1975), S. 167, 223 u. 337; vgl. Moser JB (1842) Das Wiener Volksleben in komischen Scenen mit eingelegten Liedern [...]. 2. Band. Der politische Schneider und der phlegmatische Schuster. Mörschner, Wien. S. IV.

${ }_{41}$ Reith R (1999) Lohn und Leistung. Lohnformen im Gewerbe, 1450-1900. Steiner, Stuttgart. S. 190 u. $293 \mathrm{f}$.

${ }^{42}$ Harnisch W (1865) Mein Lebensmorgen. Nachgelassene Schrift von Wilhelm Harnisch. Zur Geschichte der Jahre 1787-1822. Hrsg. von H. E. Schmieder. Verlag von Wilhelm Hertz, Berlin. S. 20 u. 23.
} 
mehrheitlich den labouring poor zugerechnet werden, darauf deuten Steuerleistungen und die Preise für Reparaturen hin (vgl. Tabelle 3). ${ }^{43}$

Erwähnenswert erscheint der Umstand, dass Ausbesserungs- und Umarbeiten offenbar - ganz analog zum Handel mit Gebrauchtem - eine Nische bildeten, in der partiell auch Angehörige ethnischer oder religiöser Minoritäten tätig werden konnten. Hier sei nur kurz das Beispiel der Roma und Sinti angeführt: Diese waren vielfach als Reparaturdienstleister tätig; häufig beschäftigten sie sich mit dem Ausbessern von Metallwaren, was in Südosteuropa mitunter bis heute noch anzutreffen ist. ${ }^{44}$ Erstaunlicherweise scheinen die Tätigkeitsfelder einiger ,nichtzigeunerischer' Gruppen in Westeuropa denen der Roma und Sinti partiell zu ähneln: Etwa der niederländischen Reizigers (Reisende), die als Halb-Sesshafte eine Mischung aus Wanderhandel und Reparaturhandwerk betrieben oder der Tinkers (Kesselflicker), die bis ins 20. Jahrhundert Reparaturtätigkeiten in Irland und Schottland ambulant ausübten. ${ }^{45}$

Die Veränderungen des Reparierens während der Industrialisierung sind noch kaum bearbeitet worden: Einerseits gab es erhebliche Persistenzen bis ins 20. Jahrhundert, andererseits verloren Reparaturen durch sinkende Preise (vor allem bei Bekleidung) sukzessive an Bedeutung. Gleichzeitig wurden viele Handwerker zu Reparaturdienstleistern, etwa Schneider oder Schuhmacher, und es entstanden im industriellen Bereich neue Reparaturberufe (hier wären die Berufe des Schlossers und des Mechanikers zu nennen). ${ }^{46}$ Und - à la longue - veränderten die Ersatzteile das Reparieren. ${ }^{47}$

\footnotetext{
43 Die Schuhflicker am Wiener Tandelmarkt bezahlten etwa in den 1770er und 1780er Jahren pro Jahr größtenteils $2 \mathrm{fl}$. - dies ist signifikant weniger, als die anderen Anbieter auf diesem Markt entrichten mussten und entspricht der Steuerleistung für „Mitleydige“, das heißt verarmte, Bürger (Wiener Stadt- und Landesarchiv, Steueramt, B10/7-26 [Kataster 1772-1791]); in den österreichischen Ländern wurden den Flickschneidern zum Ende der 1850er Jahre die niedrigste Steuergruppe zugwiesen. Höfken G (1860) Die Reform der direckten Besteuerung in Oesterreich auf Grund der Anträge des k. k. Finanzministeriums. K. K. Hof- und Staatsdruckerei, Wien. o. S. [11. Abschnitt „Begründung des Gesetzes über die Erwerbssteuer"?.

${ }_{44}$ Fricke T (1996) Zigeuner im Zeitalter des Absolutismus. Bilanz einer einseitigen Überlieferung. Eine sozialgeschichtliche Untersuchung anhand süddeutscher Quellen. Centaurus-Verlagsgesellschaft, Pfaffenweiler. S. 425f. u. 461f.; Grellmann HMG (1787) Historischer Versuch über die Zigeuner, betreffend die Lebensart und Verfassung, Sitten und Schicksale dieses Volks seit seiner Erscheinung in Europa, und dessen Ursprung. Zweyte, viel veränderte und vermehrte Auflage. Johann Christian Dieterich, Göttingen. S. 82-84.

${ }^{45}$ Faber MH (1983) Nichtzigeunerische Landfahrer in Deutschland und anderen europäischen Ländern. In: R. Vossen (ed) Zigeuner. Roma, Sinti, Gitanos, Gypsies zwischen Verfolgung und Romantisierung. Katalog zur Ausstellung „Zigeuner zwischen Romantisierung und Verfolgung - Roma, Sinti, Manusch, Calé in Europa“ des Hamburgischen Museums für Völkerkunde. Ullstein, Frankfurt a. M. et al. S. 187-203. S. 194 u. 198 f.

${ }^{46}$ Enzyklopädie (2010), Bd. 11, Sp. 58-61, s. v. Reparatur; vgl. Mayhew (1861), S. 34f.

${ }^{47}$ Reith R (2002) Reparieren: Ein Thema der Technikgeschichte? In: R. Reith, D. Schmidt (ed) Kleine Betriebe - angepasste Technologie? Waxmann, Münster, New York. S. 139-161. S. 141f.; vgl. dazu auch das Themenheft Technikgeschichte 79/3 (2013) zum Reparieren.
} 


\section{Wiederverwerten}

„Thrum ist eigentlich ein Faden Garn, oder vielmehr das Ende davon. Auch für diese Armseligkeiten finden sich in London, wo nichts umkommt, Abnehmer; und ganze Botiquen von dem, was als Individuum mit Recht als werthlos in den Staub getreten, als Aggregat aber ein Gegenstand des Handels wird. Man braucht es z.E. beym Schiffbau, um die Fugen damit zu verstopfen, u.s.w.“48

Die vormoderne Nutzung von Materialien differierte entscheidend von ihrer modernen beziehungsweise postmodernen: Materialien wurden wiederverwertet, $\mathrm{Ne}$ ben- und Beiprodukte verwendet, ${ }^{49}$ Stoffzirkulationen waren ausgeprägt und Abfall in einem modernen Sinn existierte kaum. ${ }^{50}$ Im Folgenden will ich mich jedoch auf die Wiederverwertung von Materialien beschränken, die in die Produktion neuer Güter einflossen.

Auf den hohen Wert vieler Materialien in der vorindustriellen Zeit wurde bereits verwiesen, zudem waren viele Rohstoffe knapp und manche Produkte basierten auf Recycling, wie etwa Papier, das bis ins ausgehende 19. Jahrhundert aus alttextilen Resten und Altpapier hergestellt wurde. Wiederverwerten konnte also helfen Ausgaben zu reduzieren oder knappe Rohstoffe bereitstellen. Unterstrichen wird das Ausmaß vormodernen Wiederverwertens durch die geringe Anzahl von Glas- oder Metallfunden durch die Mittelalter- und Frühneuzeitarchäologie, auf dessen Relevanz deuten regelmäßige Konflikte hin. ${ }^{51}$

Wiederverwertet wurde im häuslichen wie gewerblichen Bereich: Haushalte sammelten Altmaterialien für häusliche Zwecke - etwa Stoffreste für Ausbesserungsarbeiten, als Putzutensil, Zunder oder sogar als Verbandsmaterial - oder um diese an Sammler/innen weiterzugeben, die partiell dafür bezahlten oder Materialien (vor allem Textilreste, Metalle und Glas) gegen geringwertige Neuwaren (Nadeln, Bänder oder Süßigkeiten) tauschten. ${ }^{52}$ Im Gewerbe war die Wiederverwertung von Materialien ausgeprägt, vor allem bei Metallen, Glas oder Baumaterialien. ${ }^{53}$ Teilweise fungierten die Handwerker selbst als ,Sammler' von Altmaterialien - einige Produzenten nahmen Gebrauchtes als nichtmonetären Teil bei der Bezahlung von Neuwaren oder sie griffen auf spezialisierte Sammler/innen zurück. ${ }^{54}$

\footnotetext{
48 Colquhoun P (1800) Ueber Londons Polizey [...]. 1. Teil. Baumgärtnerschen, Leipzig. S. XXIIf.

49 Woodward D (1985) „Swords into Ploughshares“. Recycling in Pre-Industrial England. In: The Economic History Review. New Series 38. S. 175-191; Woodward D (1998) Straw, Bracken and the Wicklow Whale. The Exploitation of Natural Resources in England since 1500. In: Past and Present 159. S. 43-76; Barles S (2005) A Metabolic Approach to the City. 19th and 20th Century Paris. In: D. Schott, B. Luckin, G. Massard-Guilbaud (ed) Resources of the City. Contributions to an Environmental History of Modern Europe. Ashgate, Aldershot. S. 28-47.

${ }^{50}$ Enzyklopädie (2005), Bd. 1, Sp. 11-13, s. v. Abfall.

51 Groebner (1993), S. 181f.; Reith (2003), S. 48.

${ }^{52}$ Ratcliffe BM (1992) Perceptions and Realities of the Urban Margin. The Rag Pickers of Paris in the First Half of the Nineteenth Century. In: Canadian Journal of History 27, Nr. 2. S. 197-233; Mayhew (1861), S. 103 f. u. 107.

53 Woodward (1985); Barles (2005), S. 29 f.

${ }^{54}$ Reith (2003), S. 56 f.
} 
Bedeutend war die Wiederverwertung von Alttextilien, darüber ist auch am meisten bekannt: Altkleidung wurde vielfach umgearbeitet und weiterverkauft (vgl. Punkte 3 und 4), am Ende der Nutzung als Kleidung führte man die Stoffreste als Lumpen (im österreichischen Sprachraum auch als „Strazzen“ oder „Hadern“ bezeichnet) der Papierproduktion oder anderen Zwecken zu, eine Praxis, die auch in städtischen Institutionen anzutreffen war. Da Lumpen in der vorindustriellen Papierproduktion nicht substituiert werden konnten, führte der stetige Anstieg des Papierverbrauchs während der Frühen Neuzeit zu einem periodisch auftretenden Mangel an Lumpen. Dementsprechend wurde das Lumpensammeln vielerorts schon früh beschränkt und informelles Sammeln untersagt, den Papiermühlen wurden eigene Sammelbezirke zugewiesen und die territorialen Obrigkeiten sprachen Exportverbote aus. Dennoch bestand ein erheblicher überregionaler Lumpenhandel, vor allem qualitativ höherwertige Leinenlumpen wurden stark nachgefragt. ${ }^{55}$

Zentral für die Wiederverwertung waren spezialisierte Sammler/innen, deren Tätigkeit vom Aufkaufen bis zum ,reinen'Sammeln nahe am Bettel reichen konnte. Die Sammler/innen waren teilweise konzessioniert (vor allem bei Lumpen), in der Regel aber informell tätig. Obgleich vielfach als „Lumpensammler“, im Englischen als rag picker oder rag and bone men, im Französischen als chiffonniers, bezeichnet, scheinen eher allgemeine Sammlungstätigkeiten verbreitet gewesen zu sein, die auch Lebensmittelreste oder Holzstücke umfassen konnten. ${ }^{56}$ Am Land, mitunter auch in der Stadt, fungierten Wanderhändler und Hausierer als Aufkäufer von Altmaterialien - diese tauschten partiell Neuwaren gegen Altmaterialien. ${ }^{57}$

\footnotetext{
55 Bayerl G (1987) Die Papiermühle. Vorindustrielle Papiermacherei auf dem Gebiet des alten deutschen Reiches - Technologie, Arbeitsverhältnisse, Umwelt. P. Lang, Bern et al. S. 370-379; Warnecke HJ (2004) Lumpensammler aus Ochtrup. Quellen zu einem Wandergewerbe im Westmünsterland und in der Grafschaft Bentheim (17.-19. Jh.). Rheinisch-westfälische Zeitschrift für Volkskunde 49. S. 203-227; Stöger (2011), S. 20f. u. 44.

56 Ratcliffe (1992), S. 222; Mayhew (1861), S. 136-141.

${ }^{57}$ Warnecke (2004).
} 


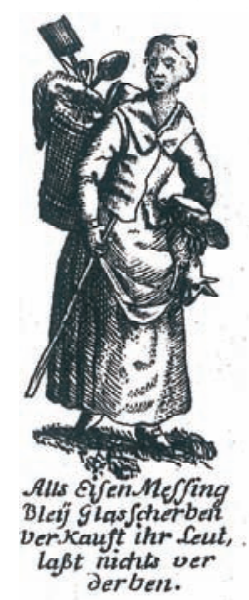

Abb. 2: Altmaterialsammlerin, Österreich 1780er (aus: Anonymus (1786), unpaginiert).

In der Regel müssen Sammeltätigkeiten als Ausdruck von Armut, als survial strategies der labouring poor gedeutet werden. Dementsprechend konnten sie auch als Erwerbstätigkeit für Migranten, Exkludierte oder ältere, partiell arbeitsunfähige Menschen fungieren. Zudem scheint die Bedeutung von Frauen- und Kinderarbeit in diesem Bereich - als Sammler/innen, aber auch in damit verbundenen Tätigkeiten (dem Sortieren und Aufbereiten von Materialien) - hoch gewesen zu sein. ${ }^{58}$ Bildliche Quellen, die vor allem des 18. und beginnenden 19. Jahrhunderts zeigen vielfach Frauen, die stets ärmlich gekleidet sind (vgl. auch Abb. 2). ${ }^{59}$

Die Arbeitsbedingungen der Sammler/innen zeichnen Gewerbepathologien und frühe Sozialstudien nach: Durch den Umgang mit Abfällen bestanden erhebliche Gesundheitsgefährdungen - einerseits durch Staub (besonders beim Aufbereiten von Lumpen), andererseits war die Gefahr von Infektionen omnipräsent. ${ }^{60}$ Die unhygienischen Bedingungen, die diese Tätigkeiten meist begleiteten, auch die grundlegende Armut der Sammler/innen und die ambulante Natur des Sammelns begünstigten negative zeitgenössische Deutungen. Vielfach wurden angebliche Berührungspunkte mit kriminellen und devianten Bereichen hergestellt, gleichzeitig kam es in der Populärkultur - vor allem ab dem beginnenden 19. Jahrhundert mitunter zu romantisierenden beziehungsweise exotischen Projektionen. ${ }^{61}$

\footnotetext{
${ }^{58}$ Faure A (1996) Sordid Class, Dangerous Class? Observations on Parisian Ragpickers and Their Cités During the Nineteenth Century. In: International Review of Social History 41. S. 157-176. Hier S. 160-162; Ratcliffe (1992), S. 200 u. 218f.; Woodward (1985), S. 189; Bayerl (1987), S. 347; Colquhoun (1800), S. XXXII, 92f. u. 181.

${ }^{59}$ Beall (1975), S. 97, 103 u. 469.

${ }^{60}$ Bayerl (1987), S. 357-360.

${ }^{61}$ Colquhoun (1800), S. 55 u. 181; Ratcliffe (1992), S. 205-207.
} 
Auch beim Wiederverwerten ist eine Annäherung an die Akteure schwierig, einerseits, da es sich um ,mindere' Erwerbsbereiche handelt, andererseits, da Sammler oft informell agierten. Schon die Anzahl der Sammler/innen ist weitgehend unklar. Es gibt nur - wenig zuverlässig erscheinende - Schätzungen: Zur Mitte des 19. Jahrhunderts soll es in Paris über 2.000 chiffonniers (also Sammler) gegeben haben ${ }^{62}$, in den österreichischen Territorien „mehr als 10.000 Hadernsammler“63, im Jahre 1838 wurden die 52 hannoverische Papiermühlen von ungefähr 1.200 Sammlern/innen mit Lumpen versorgt. ${ }^{64}$ Dies sind beeindruckende Zahlen, auch die benötigten beziehungsweise gesammelten Materialmengen sind alles andere als marginal: Der jährliche Bedarf an Lumpen in den Papiermühlen der österreichischen Territorien belief sich in den 1840er Jahren auf 804.000 Zentner (das heißt etwa 40.000 Tonnen! ${ }^{65}$, in den 1850 er Jahren erreichten jährlich etwa 10.000 Tonnen Leinenlumpen - mehrheitlich aus deutschen und mediterranen Häfen Großbritannien, welche zu einem erheblichen Teil nach Nordamerika exportiert wurden $^{66}$, bis in die 1860 er Jahre hatte sich die Menge mehr als verdoppelt. ${ }^{67}$

Wie veränderte sich das Wiederverwerten während der Industrialisierung? Dies ist ebenso noch weitgehend unklar, viele Hinweise sprechen aber für Persistenzen vormoderner Recyclingsysteme, die bis in die zweite Hälfte des 19. Jahrhunderts bestanden: Das Sammeln blieb eine wichtige Erwerbsmöglichkeit für manche Angehörige der labouring poor, langsam sinkende Rohstoffpreise begünstigten das Wiederverwerten, auch kam es infolge der Industrialisierung zu einer Neunutzung von Altmaterialien ${ }^{68}$ und partiell zu einer verstärkten Nachfrage nach Altstoffen, etwa in der Papierproduktion, in der sich Holz als Rohstoff erst zum Ende des Jahrhunderts durchsetzen konnte. So prosperierte der internationale Lumpenhandel (vgl. auch oben): Im Jahre 1850 kamen beispielsweise fast 50.000 Tonnen europäische Lumpen in Nordamerika an. ${ }^{69}$ Die sich in den letzten Jahrzehnten des 19. Jahrhunderts intensivierenden Hygienisierungsbestrebungen, die die Einrichtung zentralisierter Entsorgungssysteme durch die Kommunen mit sich brachte, zudem das Absinken der Rohstoffpreise, bedingten eine sukzessive Marginalisierung, traditioneller' Stoffkreisläufe und Verwertungssysteme. In der westlichen Welt verlor die Wiederverwertung in der ersten Hälfte des 20. Jahrhunderts allge-

\footnotetext{
62 Ratcliffe (1992), S. $215 f$.

${ }^{63}$ Reden FW v. (1846) Denkschrift über die österreichische Gewerbe-Ausstellung in Wien 1845 [...]. Schröder, Berlin. S. 62

${ }^{64}$ Laufer J (2008) Knappe Ressourcen als Barriere und Triebkraft innovativer Entwicklung: Zur Bedeutung von Lumpen, Holz und Wasser in der niedersächsischen Papierindustrie (19./20. Jahrhundert). In: Niedersächsisches Jahrbuch für Landesgeschichte 80. S. 215-240. Hier S. 219.

${ }^{65}$ Reden (1846), S. 62.

${ }^{66}$ McCulloch JR (1850) A Dictionary, Practical, Theoretical, and Historical, of Commerce and Commercial Navigation. Longman, Brown, Green and Longmans, London. S. $1088 \mathrm{f}$.

67 Anonymus (1866) Commerce. Journal of the Society of Arts, 5. Oktober 1866. S. 717.

${ }^{68}$ Ein Beispiel hierfür ist shoddy, eine seit der Mitte des 19. Jahrhunderts bestehende Kunstwolle, die aus Wolllumpen erzeugt wurde - vgl. Strasser S (1999) Waste and want. A social history of trash. Metropolitan Books, New York. S. 93-97.

${ }^{69}$ Ebd., S. 83f.
} 
mein stark an Bedeutung - als Zäsur sind hier, Christian Pfister folgend, aber vermutlich erst die 1950er Jahre anzusehen. ${ }^{70}$

\section{Zusammenfassung}

Die bisher vorliegenden Befunde unterstreichen die Bedeutung sekundärer Märkte und Transfers für die vormoderne Wirtschaft und Gesellschaft im Hinblick auf die Konsumption wie auf die Beschäftigung. Wenngleich auch mittlere und höhere Schichten an derartigen Märkten und Zirkulationen partizipierten, ist deren Stellenwert als Erwerbsmöglichkeit und Konsumptionsstrategie für die labouring poor, besonders für Frauen und Angehörige ethnischer oder religiöser Minoritäten, als sehr hoch einzuschätzen.

Es gab einen spezifischen vormodernen „Umgang mit Sachen“, der sich infolge von spezifischen Konstellationen ausgeprägt hatte: Einerseits war er ökonomisch induziert - da Gegenstände und Materialien einen hohen Wert aufwiesen und Haushalte wie Individuen in sozioökonomischer Hinsicht nur unzureichend abgesichert waren, andererseits hatte sich infolge der Gewissheit periodisch wiederkehrender Krisen ein sorgfältiger und sparsamer Umgang mit Ressourcen als Grundzug des Wirtschaftens ausgeprägt.

Wenn derartige Bereiche in der Forschung keine Berücksichtigung finden, kann dies verschiedene Probleme mit sich bringen: Es führt zu einem Ausblenden wichtiger Haushaltstrategien und signifikanter Zirkulationen, die vor allem - aber nicht nur - ein erhebliches Beschäftigungspotential auswiesen. Beim Ausklammern der Wiederverwertung kann es beispielsweise zu einem quantitativen Unterschätzen der Produktion insgesamt kommen, wenn - wie oftmals praktiziert - nur der Input von Primärmaterialien als Indikator herangezogen wird und damit Altstoffe nicht beachtet werden. ${ }^{71}$

Es bestehen aus meiner Sicht in diesem Themenbereich erhebliche Forschungslücken: Viele Aspekte des Wiederverwertens und Weiternutzens und anderer ,sekundärer' Praktiken und Zirkulationen in der Vormoderne sind noch völlig unklar, insbesondere der ländliche Raum ist diesbezüglich kaum bearbeitet worden. Wir wissen zudem relativ wenig über die Entwicklung sekundärer Märkte im 19. und 20. Jahrhundert, also über Persistenzen und Veränderungen durch Industrialisierung, die ich kurz in den jeweiligen Abschnitten angesprochen habe. Ein globaler Blick, dies betrifft vor allem das 20. Jahrhundert, könnte auch wesentliche Einblicke gewähren und unseren Blick auf Entstehen und Entwicklung der Wegwerfgesellschaft schärfen. ${ }^{72}$ Hier könnte der Ansatz der commodity chains, der den

\footnotetext{
70 Pfister (1995); Barles (2005), S. $43 \mathrm{f}$.

71 Woodward (1985), $190 f$.

72 Tranberg Hansen K (2000) Salaula. The World of Secondhand Clothing and Zambia. University of Chicago Press, Chicago, London; Gerasimova E, Chuikina S (2009) The Repair Society. Russian Studies in History 48, Nr. 1. S. 58-74.
} 
,Lebenszyklus‘ eines Produktes verfolgt (was sekundäre Nutzungsformen und schlussendlich auch das Wegwerfen einschließen würde) ${ }^{73}$, einen Zugang und einen Anknüpfungspunkt zu weiteren Diskursen liefern.

\section{Literatur}

Ago R (2008) Using Things as Money: An Example from Later Renaissance Rome. In: L. Fontaine (ed) Alternative exchanges. Second-hand circulations from the sixteenth century to the present. Berghahn Books, New York, Oxford. S. 43-60.

Allerston P (2000) Clothing and early modern Venetian society. In: Continuity and Change 15, Nr. 3. S. 367-390.

Anonymus (1494) Ein liepliche histori und warheit von vir kaufmendern. S. n., s. 1. (Nürnberg) - Onlinefaksimile: http://daten.digitalesammlungen.de/ db/0002/bsb00027693/images.

Anonymus (s.a. [1786]) Neuer Krakauer Finger-Kalender auf das Jahr 1787 [...], Trattner, Wien.

Anonymus (1866) Commerce. Journal of the Society of Arts, 5. Oktober 1866. S. 717.

Barles S (2005) A Metabolic Approach to the City. 19th and 20th Century Paris. In: D. Schott, B. Luckin, G. Massard-Guilbaud (ed) Resources of the City. Contributions to an Environmental History of Modern Europe. Ashgate, Aldershot. S. 28-47.

Bayerl G (1987) Die Papiermühle. Vorindustrielle Papiermacherei auf dem Gebiet des alten deutschen Reiches - Technologie, Arbeitsverhältnisse, Umwelt. P. Lang, Bern et al.

Beall KF (1975) Kaufrufe und Strassenhändler. Cries and itinerant trades. Eine Bibliographie. A bibliography. Hauswedell, Hamburg.

Charpy M (2008) The Scope and Structure of the Nineteenth-century Second-hand Trade in the Parisian Clothes Market. In: L. Fontaine (ed) Alternative exchanges. Second-hand circulations from the sixteenth century to the present. Berghahn Books, New York, Oxford. S. 127-151.

Coffin JG (1994) Gender and the Guild Order: The Garment Trades in Eighteenth-Century Paris. In: The Journal of Economic History 54. S. 768793.

\footnotetext{
${ }^{73}$ Dieser Ansatz kommt zwar aus der Betrachtung globaler Transfers, er eignet sich meines Erachtens jedoch ebenso für lokale oder regionale flows - vgl. Van der Linden M (2006) The „Globalization" of Labour and Working-Class History and Its Consequences. In: J. Lucassen (ed) Global Labour History. A State of the Art. P. Lang, Bern, New York. S. 13-36.
} 
Colquhoun P (1800) Ueber Londons Polizey [...]. 1. Teil. Baumgärtnerschen, Leipzig.

Deceulaer H (2008) Second-hand Dealers in the Early Modern Low Countries: Institutions, Markets and Practices. In: L. Fontaine (ed) Alternative exchanges. Second-hand circulations from the sixteenth century to the present. Berghahn Books, New York, Oxford. S. 13-42.

E. I. C. P. N. (1682) Die Kluge Trödel-Frau. Worinnen enthalten Allerhand Listige Kunst-Griffe. Dadurch der Eigennutz heutiges Tages von Jederman gesuchet wird. S. n., s. 1.

Enzyklopädie der Neuzeit (2005ff). Hrsg. von F. Jäger, 16 Bde., J. B. Metzler, Stuttgart, Weimar.

Faber MH (1983) Nichtzigeunerische Landfahrer in Deutschland und anderen europäischen Ländern. In: R. Vossen (ed) Zigeuner. Roma, Sinti, Gitanos, Gypsies zwischen Verfolgung und Romantisierung. Katalog zur Ausstellung „Zigeuner zwischen Romantisierung und Verfolgung - Roma, Sinti, Manusch, Calé in Europa" des Hamburgischen Museums für Völkerkunde. Ullstein, Frankfurt a. M. et al. S. 187-203.

Faure A (1996) Sordid Class, Dangerous Class? Observations on Parisian Ragpickers and Their Cités During the Nineteenth Century. In: International Review of Social History 41. S. 157-176.

Fontaine L (2002) Women's Economic Spheres and Credit in Pre-industrial Europe. In: B. Lemire et al. (ed) Women and Credit. Researching the Past, Refiguring the Future, Berg, Oxford, New York. S. 15-32.

Fontaine L (2011) Märkte als Chance für die Armen in der Frühen Neuzeit. In: Zeitschrift für Agrargeschichte und Agrarsoziologie 59, Nr. 2. S. 37-53.

Fontaine L, Schlumbohm J (2000) Household Strategies for Survival: An Introduction. In: International Review of Social History 45. S. 1-17.

Fouquet G (2012) Sparsamkeit - ein Phänomen des Haushaltens in den Lebenswelten des Mittelalters. Harald Witthöft zum 80. Geburtstag. In: Vierteljahrschrift für Sozial- und Wirtschaftsgeschichte 99. S. 1-15.

Fricke T (1996) Zigeuner im Zeitalter des Absolutismus. Bilanz einer einseitigen Überlieferung. Eine sozialgeschichtliche Untersuchung anhand süddeutscher Quellen. Centaurus-Verlagsgesellschaft, Pfaffenweiler.

Füssel M (2009) Der Wert der Dinge. Materielle Kultur in soldatischen Selbstzeugnissen des Siebenjährigen Krieges. In: Militär und Gesellschaft in der Frühen Neuzeit 13, Nr. 1. S. 104-121. 
Gerasimova E, Chuikina S (2009) The Repair Society. Russian Studies in History 48, Nr. 1. S. 58-74.

Glass C (1983), Reparierendes Handwerk. In: Ludwig-Uhland-Institut f. Emp. Kulturwiss. der Univ. Tübingen, Württemberg. Landesmuseum Stuttgart (ed) Flick-Werk. Reparieren und Umnutzen in der Alltagskultur. Begleitheft zur Ausstellung im Württembergischen Landesmuseum Stuttgart vom 15. Oktober bis 15. Dezember 1983. Württembergisches Landesmuseum, Stuttgart. S. 35-42.

Grellmann HMG (1787) Historischer Versuch über die Zigeuner, betreffend die Lebensart und Verfassung, Sitten und Schicksale dieses Volks seit seiner Erscheinung in Europa, und dessen Ursprung. Zweyte, viel veränderte und vermehrte Auflage. Johann Christian Dieterich, Göttingen.

Groebner V (1993) Ökonomie ohne Haus. Zum Wirtschaften armer Leute in Nürnberg am Ende des 15. Jahrhunderts. Vandenhoeck \& Ruprecht, Göttingen.

Harnisch W (1865) Mein Lebensmorgen. Nachgelassene Schrift von Wilhelm Harnisch. Zur Geschichte der Jahre 1787-1822. Hrsg. von H. E. Schmieder. Verlag von Wilhelm Hertz, Berlin.

Heilig B (1931), Die Vorläufer der mährischen Konfektionsindustrie in ihrem Kampf mit den Zünften. In: Jahrbuch der Gesellschaft für Geschichte der Juden in der Čechoslovakischen Republik 3. S. 307-448.

Höfken G (1860) Die Reform der direckten Besteuerung in Oesterreich auf Grund der Anträge des k. k. Finanzministeriums. K. K. Hof- und Staatsdruckerei, Wien.

Holbach R (1991) „Im auff Arbeit gelihen“. Zur Rolle des Kredits in der gewerblichen Produktion vom Mittelalter bis ins 16. Jahrhundert. In: M. North (ed) Kredit im spätmittelalterlichen und frühneuzeitlichen Europa. Böhlau, Köln, Wien. S. 133-158.

Hornschuch F (1930) Aufbau und Geschichte der interterritorialen Kesslerkreise in Deutschland. Kohlhammer, Stuttgart.

Hufton O (1974) The Poor of Eighteenth-century France 1750-1789. Clarendon Press, Oxford.

Internationale Stiftung Mozarteum Salzburg (2005) Mozart. Briefe und Aufzeichnungen. Gesamtausgabe. Bd. 3. Bärenreiter, Kassel et al.

Jeggle U (1983) Vom Umgang mit Sachen. In: K. Köstlin, H. Bausinger (ed) Umgang mit Sachen. Zur Kulturgeschichte des Dinggebrauchs. 23. Deutscher Volkskundekongreß in Regensburg vom 6.-11. Oktober 1981. Univ. Regensburg, Regensburg. S. 11-25. 
Küchelbecker JB (1730) Allerneueste Nachricht vom Römisch-Kayserlichen Hof [...]. Förster, Hannover.

Laufer J (2008) Knappe Ressourcen als Barriere und Triebkraft innovativer Entwicklung: Zur Bedeutung von Lumpen, Holz und Wasser in der niedersächsischen Papierindustrie (19./20. Jahrhundert). In: Niedersächsisches Jahrbuch für Landesgeschichte 80. S. 215-240.

Lemire B (1997) Dress, culture and commerce. The English clothing trade before the factory, 1660-1800. Macmillan, Basingstoke.

Mayhew H (1861) London Labour and the London poor. [...]. Bd. 2. Griffin u. Bohn, London.

McCants AEC (2007) Goods at Pawn: The Overlapping Worlds of Material Possessions and Family Finance in Early Modern Amsterdam. In: Social Science History 31. S. 213-238.

McCulloch JR (1850) A Dictionary, Practical, Theoretical, and Historical, of Commerce and Commercial Navigation. Longman, Brown, Green and Longmans, London.

Moser JB (1842) Das Wiener Volksleben in komischen Scenen mit eingelegten Liedern [...]. 2. Band. Der politische Schneider und der phlegmatische Schuster. Mörschner, Wien.

Pennell S (2012) Material culture in seventeenth-century ,Britain'. The matter of domestic consumption. In: F. Trentmann (ed) The Oxford handbook of the history of consumption. Oxford University Press, Oxford. S. 64-84.

Pfister C (2010) The „1950s Syndrome“ and the Transition from a Slow-Going to a Rapid Loss of Global Sustainability. In: F. Uekötter (ed) The Turning Points of Environmental History. University of Pittsburgh Press, Pittsburgh. S. 90-118.

Pfister C et al. (1995) „Das 1950er Synrom“: Zusammenfassung und Synthese. In: C. Pfister (ed) Das 1950er Syndrom. Der Weg in die Konsumgesellschaft. Haupt, Bern et al. S. 21-47.

Ratcliffe BM (1992) Perceptions and Realities of the Urban Margin. The Rag Pickers of Paris in the First Half of the Nineteenth Century. In: Canadian Journal of History 27, Nr. 2. S. 197-233.

Reden FW v. (1846) Denkschrift über die österreichische Gewerbe-Ausstellung in Wien 1845 [...]. Schröder, Berlin.

Reith R (1998) Praxis der Arbeit. Überlegungen zur Rekonstruktion von Arbeitsprozessen in der handwerklichen Produktion. In: R. Reith (ed) Praxis der Arbeit. Campus, Frankfurt a. M., New York. S. 11-54. 
Reith R (1999) Lohn und Leistung. Lohnformen im Gewerbe, 1450-1900. Steiner, Stuttgart.

Reith R (2002) Reparieren: Ein Thema der Technikgeschichte? In: R. Reith, D.

Schmidt (ed) Kleine Betriebe - angepasste Technologie? Waxmann, Münster, New York. S. 139-161.

Reith R (2003) Recycling im späten Mittelalter und der frühen Neuzeit. Eine Materialsammlung. Frühneuzeit-Info 14. S. 47-65.

Reuter C (1961) Graf Ehrenfried. Abdruck der Erstausgabe von 1700. Hrsg. von W. Hecht. Niemeyer, Tübingen.

Roche D (1994) The culture of clothing. Dress and fashion in the „ancien régime“. Cambridge University Press, Cambridge, New York.

Scheffknecht W (2001) Fremde Wanderkrämer und Keßler in der Grafschaft Hohenems und im Reichshof Lustenau. In: M. Häberlein, M. Zürn (ed) Minderheiten, Obrigkeit und Gesellschaft in der Frühen Neuzeit. Integrationsund Abgrenzungsprozesse im süddeutschen Raum. Scripta Mercaturae, St. Katharinen. S. 233-267.

Shesgreen S (2002) Images of the Outcast. The Urban Poor in the Cries of London. Manchester University Press, Manchester.

Stifter A (1844) Der Tandelmarkt. In: Wien und die Wiener in Bildern aus dem Leben. [...]. Heckenast, Pesth. S. 227-241.

Stobart J (2006) Clothes, Cabinets and Carriages: Second-hand Dealing in Eighteenth-century England. In: B. Blondé (ed) Buyers and Sellers. Retail Circuits and Practices in Medieval and Early Modern Europe. Brepols, Turnhout. S. 225-244.

Stöger G (2011) Sekundäre Märkte? Zum Wiener und Salzburger Gebrauchtwarenhandel im 17. und 18. Jahrhundert. Verl. für Geschichte und Politik. Oldenbourg, Wien, München.

Storey T (2008) Prostitution and the Ciruclation of Second-hand Goods in Early Modern Rome. In: L. Fontaine (ed) Alternative exchanges. Second-hand circulations from the sixteenth century to the present. Berghahn Books, New York, Oxford. S. 61-75.

Strasser S (1999) Waste and want. A social history of trash. Metropolitan Books, New York.

Styles J (2007) The Dress of the People. Everyday Fashion in Eighteenth-century England. Yale University Press, New Haven, London.

Tebutt M (1984) Making ends meet. Pawnbroking and working-class credit. Methuen, London. 
Tranberg Hansen K (2000) Salaula. The World of Secondhand Clothing and Zambia. University of Chicago Press, Chicago, London.

Van Damme I (2010) Second-Hand Dealing in Bruges and the Rise of an ,Antiquarian Culture', c. 1750-1870. In: J. Stobart, I. Van Damme (ed) Modernity and the Second-Hand Trade European Consumption Cultures and Practices, 1700-1900. Palgrave Macmillan, Houndmills. S. 73-92.

Van Damme I, Vermoesen R (2009) Second-hand Consumption as a Way of Life: Public Auctions in the Surroundings of Alost in the Late Eighteenth Century. In: Continuity and Change 24, Nr. 2. S. 275-305.

Van der Linden M (2006) The „Globalization“ of Labour and Working-Class History and Its Consequences. In: J. Lucassen (ed) Global Labour History. A State of the Art. P. Lang, Bern, New York. S. 13-36.

Warnecke HJ (2004) Lumpensammler aus Ochtrup. Quellen zu einem Wandergewerbe im Westmünsterland und in der Grafschaft Bentheim (17.-19. Jh.). Rheinisch-westfälische Zeitschrift für Volkskunde 49. S. 203-227.

Weigel C (1698) Abbildung Der Gemein-Nützlichen Haupt-Stände [...]. s. n., Regensburg.

Welch E (2012) Sites of Consumption in Early Modern Europe. In: F. Trentmann (ed) The Oxford handbook of the history of consumption. Oxford University Press, Oxford. S. 229-250.

Woodward D (1985) „Swords into Ploughshares“. Recycling in Pre-Industrial England. In: The Economic History Review. New Series 38. S. 175-191.

Woodward D (1998) Straw, Bracken and the Wicklow Whale. The Exploitation of Natural Resources in England since 1500. In: Past and Present 159. S. 43-76.

Zander-Seidel J (1990) Textiler Hausrat. Kleidung und Haustextilien in Nürnberg von 1500-1650. Deutscher Kunstverlag, München. 



\section{Die Autoren}

Gero Bauer ist Doktorand und Lehrbeauftragter für Neuere Englische Literatur an der Eberhard Karls Universität Tübingen und Visiting Research Associate am King's College London.

Jens Beck, Dr. rer. hort., geb. 1965, Studium der Architektur in Darmstadt und der Landschafts- und Freiraumplanung in Hannover. Referent für Gartendenkmalpflege im Denkmalschutzamt Hamburg. Wichtigste Veröffentlichungen der letzten Jahre: Gärten und Parks auf Rügen, Rostock 2013; Form follows function Anlage, Organisation und Gestaltung früher Baumschulen. In: Frühe Baumschulen in Deutschland, hrsg. von S. Butenschön, Berlin 2012; Adeliger Kulturtransfer zwischen Stadt und Land - Beispiele aus der Gartenkunst. In: Niedersächsisches Jahrbuch für Landesgeschichte, Bd. 84, Hannover 2012, S. 197ff.; Künstlergärten. In: Private Gartenkultur, hrsg. von der DGGL, München 2011; Die Gehölzbestände aufgelassener Friedhöfe. In: Stadt+Grün, Jg. 60, Heft 4, April 2011; Gärten und Parks in Niedersachsen mit Karl Johaentges (Fotos), Rostock 2010; Historische Gutsgärten im Elbe-Weser-Raum, Geschichte und kulturhistorische Bedeutung der Gärten als Teil der Kulturlandschaft, Stade 2009.

Philip Hahn, Dr., geb. 1980, ist wissenschaftlicher Assistent am Lehrstuhl für Neuere Geschichte (Frühe Neuzeit) der Eberhard Karls Universität Tübingen. Er hat Geschichte und Lateinische Philologie in Tübingen, Oxford (GB) und Cambridge (GB) studiert und wurde 2009 in Frankfurt/M. promoviert. Buchveröffentlichungen: Der Politik die Leviten lesen. Politik von der Kanzel in Thüringen und Sachsen, 1550-1675 (hg. zus. mit K. Paasch u. L. Schorn-Schütte, Gotha 2011); Das Haus im Buch. Konzeption, Publikationsgeschichte und Leserschaft der Oeconomia Johann Colers. Epfendorf, 2013. 
Astrid Mignon Kirchhof, Dr., ist Wissenschaftliche Mitarbeiterin am Lehrstuhl für Neueste und Zeitgeschichte der Humboldt Universität Berlin und Projektleiterin des DFG-Forschungsprojektes „Vom Natur- zum Umweltschutz. Bürgerschaftliches Engagement und der Wandel der Naturschutzbewegung in Ost- und West-Berlin 1945-1990“. Veröffentlichungen: Spanning the Globe: West-German Support for the Australian Anti-Nuclear Movement. In: A. M. Kirchhof, J.-H. Meyer (ed) Global Protest against Nuclear Power. Transfer and Transnational Exchange in the 1970s and 1980s. HSR 39 (2014) 1, p. 254-273; Frauen in der Antiatomkraftbewegung. Am Beispiel der Mütter gegen Atomkraft. In: A. M. Kirchhof, L. Schibbe (Hg.) Umweltgeschichte und Geschlecht. Von Antiatomkraftbewegung bis Ökofeminismus. (Ariadne. Forum für Frauen- und Geschlechtergeschichte, 64); Contemporary Ideas in a Traditional Mind-Set: The Nature Conservation Movement in Post War West-Germany 1945-1960. In: Ecozon@ 2 (2011) Nr.1, p. 34-47; Das Dienstfräulein auf dem Bahnhof". Frauen im öffentlichen Raum im Blick der Berliner Bahnhofsmission 1894-1939. Stuttgart 2011.

Patrick Kupper, PD Dr., ist Dozent an der ETH Zürich, Wichtigste Buchveröffentlichungen: Atomenergie und gespaltene Gesellschaft: Die Geschichte des gescheiterten Projektes Kernkraftwerk Kaiseraugst. Zürich, Chronos, 2003; Die Zukunftsmaschine: Konjunkturen der ETH Zürich 1855-2005. Zürich, Chronos, 2005 (mit David Gugerli und Daniel Speich); Wildnis schaffen: Eine transnationale Geschichte des Schweizerischen Nationalparks. Bern, Haupt, 2012; Civilizing Nature: National Parks in Global Historical Perspective. New York, Berghahn Books, 2012 (Hg. mit Bernhard Gissibl und Sabine Höhler); Geschichte des Nationalparks Hohe Tauern. Innsbruck, Tyrolia, 2013 (mit Anna-Katharina Wöbse).

Karsten Reise, Prof. Dr., war langjähriger Leiter der Wattenmeerstation Sylt des Alfred-Wegener-Instituts, Helmholtz-Zentrum für Polar- und Meeresforschung und Professor für Küstenforschung an der Universität Kiel, seit 2013 im Ruhestand, Bücher: Tidal Flat Ecology. Ecological Studies 54, Berlin, Heidelberg, Springer, 1985; Ökosystem Wattenmeer. Springer, Berlin u. Heidelberg, 1998, hg. zus. mit Christiane Gätje; Ecological comparisons of sedimentary shores. , Springer, Berlin, Heidelberg 2000; Küstenmentalität und Klimawandel. Oekom, München 2011, hg. zus, mit Ludwig Fischer; A natural history of the Wadden Sea. Leeuwarden, NL, 2013.

Martin Rheinheimer, Prof. Dr., ist Professor für Maritim und Regionalgeschichte an der Syddansk Universitet in Odense (Dänemark). Wichtigste Buchveröffentlichungen: Arme, Bettler und Vaganten. Überleben in der Not 1450-1850 (Europäische Geschichte). Frankfurt am Main, Fischer Taschenbuch Verlag, 2000; Der fremde Sohn. Hark Olufs' Wiederkehr aus der Sklaverei. Neumünster, Wachholtz Verlag, 2001; Der Kojenmann. Mensch und Natur im Wattenmeer 1860-1900. Neumünster, Wachholtz Verlag, 2007. 
Werner Rösener, Prof. Dr., war bis 2009 Professor für Mittlere und Neuere Geschichte mit dem Schwerpunkt Hochmittelalter an der Universität Gießen. Forschungsschwerpunkte im Bereich für Sozial-, Wirtschafts- und Verfassungsgeschichte und besonders der Agrargeschichte des Mittelalters und der Neuzeit.

Georg Stöger, Dr. phil., ist Assistent für Wirtschafts-, Sozial- und Umweltgeschichte am Fachbereich Geschichte der Universität Salzburg, derzeitiger Schwerpunkt im Bereich städtische Umweltgeschichte (18. und 19. Jahrhundert), neueste Publikationen: Neuanläufe für einen Nationalpark (1949-1970). In: Patrick Kupper, Anna-Katharina Wöbse (Hg.) Geschichte des Nationalparks Hohe Tauern, Innsbruck 2013, 93-119; Themenheft „Reparieren - oder die Lebensdauer der Gebrauchsgüter", Technikgeschichte 79/3, 2012 (hg. gem. mit Reinhold Reith); Sekundäre Märkte? Zum Wiener und Salzburger Gebrauchtwarenhandel im 17. und 18. Jahrhundert, Wien u. München, 2011 (als Open-Access-Publikation unter: https:/ / fedora.e-book.fwf.ac.at/fedora/get/o:206/bdef:Content/get). 
Seit seiner Gründung vor annähernd 25 Jahren hat sich das Göttinger Umwelthistorische Kolloquium zu einer Einrichtung entwickelt, welche die vielfältigen, thematisch einschlägigen Aktivitäten des Standortes wie auch des deutschsprachigen Raumes durch Austausch von Forschungsergebnissen und Sichtweisen bündelt. Von hier haben auch einige Unternehmungen ihren Ausgang genommen, welche zum heutigen Profil der Umweltgeschichte spürbar beitrugen.

Der Band vereinigt Beiträge zum Kolloquium des Sommersemesters 2011 und des Wintersemesters $2011 / 12$. 